Supporting Information For:

\title{
Multiple Electrophilic C-H Borylation of Arenes Using Boron Triiodide
}

Susumu Oda, Kenta Ueura, Bungo Kawakami, Takuji Hatakeyama*

Prof. T. Hatakeyama, Dr. S. Oda, K. Ueura, B. Kawakami

Department of Chemistry, School of Science and Technology, Kwansei Gakuin University, 2-1 Gakuen, Sanda, Hyogo 669-1337, Japan

E-mail: hatake@kwansei.ac.jp 


\section{EXPERIMENTAL SECTION}

General Procedure. All the reactions dealing with air- or moisture-sensitive compounds were carried out in a dry reaction vessel (small scale, a Schlenk flask; large scale, a three-neched round bottomed flask) under a positive pressure of nitrogen using an oil bath (below $200{ }^{\circ} \mathrm{C}$ ) or an aluminum block (above $200^{\circ} \mathrm{C}$ ). Air- and moisture-sensitive liquids and solutions were transferred via a syringe or a Teflon ${ }^{\circledR}$ cannula. Analytical thin-layer chromatography (TLC) was performed on glass plates coated

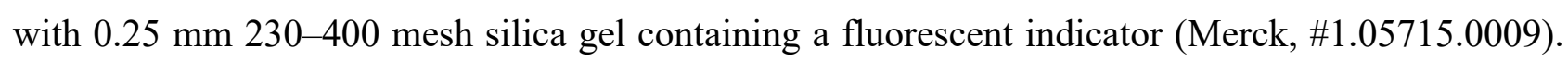
TLC plates were visualized by exposure to ultraviolet light $(254 \mathrm{~nm}$ or $365 \mathrm{~nm})$. Organic solutions were concentrated by rotary evaporation at $c a .10-50 \mathrm{mmHg}$. Flash column chromatography was performed on Merck silica gel 60 (spherical, neutral, 140-325 mesh) as described by Still et al. ${ }^{1}$ Gel permeation chromatography was performed on a JAIGEL-1H and 2H (20 mm i.d.) with an LC-9130 (Japan Analytical Industry Co., Ltd.). Proton nuclear magnetic resonance ( ${ }^{1} \mathrm{H}$ NMR), carbon nuclear magnetic resonance $\left({ }^{13} \mathrm{C} \mathrm{NMR}\right)$, boron nuclear magnetic resonance $\left({ }^{11} \mathrm{~B} \mathrm{NMR}\right)$ spectra were recorded on JEOL ECX400 (400 MHz) NMR spectrometers or JEOL ECX500 (500 MHz) NMR spectrometers. Proton chemical shift values are reported in parts per million (ppm, $\delta$ scale) downfield from tetramethylsilane and are referenced to the tetramethylsilane $(\delta 0),\left(\mathrm{CD}_{3}\right)_{2} \mathrm{CO}(\delta 2.05),\left(\mathrm{CD}_{3}\right)_{2} \mathrm{SO}(\delta$ 2.50), or $\mathrm{CDCl}_{3}\left(\delta\right.$ 7.26). ${ }^{13} \mathrm{C} \mathrm{NMR}$ spectra were recorded at $101 \mathrm{MHz}$ or $126 \mathrm{MHz}$ : carbon chemical shift values are reported in parts per million (ppm, $\delta$ scale) downfield from tetramethylsilane, and are referenced to the carbon resonance of tetramethylsilane $(\delta 0),\left(\mathrm{CD}_{3}\right)_{2} \mathrm{CO}(\delta 29.8),\left(\mathrm{CD}_{3}\right)_{2} \mathrm{SO}(\delta 39.5)$, or $\mathrm{CDCl}_{3}(\delta 77.0) .{ }^{11} \mathrm{~B}$ NMR spectra were recorded at $128 \mathrm{MHz}$ or $160 \mathrm{MHz}$ : boron chemical shift values are reported in parts per million (ppm, $\delta$ scale) and are referenced to the external standard boron signal of $\mathrm{BF}_{3} \cdot \mathrm{Et}_{2} \mathrm{O}(\delta 0) .{ }^{19} \mathrm{~F} \mathrm{NMR} \mathrm{spectra} \mathrm{were} \mathrm{recorded} \mathrm{at} 376 \mathrm{MHz}$ : fluorine chemical shift values are reported in parts per million (ppm, $\delta$ scale) and are referenced to the external standard fluorine signal of $\mathrm{CF}_{3} \mathrm{COOH}(\delta-77.6)$. Data are presented as: chemical shift, multiplicity $(\mathrm{s}=$ singlet, $\mathrm{d}=$ doublet, $\mathrm{t}=$ triplet, $\mathrm{q}=$ quartet, quint $=$ quintet, sext $=$ sextet, sept $=$ septet, $\mathrm{m}=$ multiplet and/or multiplet resonances, br = broad), coupling constant in hertz $(\mathrm{Hz})$, signal area integration in natural numbers, and assignment (italic). IR spectra were recorded on an ATR-FTIR spectrometer (FT/IR4200, JASCO or IRAffinity-1S, Shimadzu). Characteristic IR absorptions are reported in $\mathrm{cm}^{-1}$. Melting points were recorded on a Fisher-Johns 12-144-1Q melting point apparatus (according to the limitations of the apparatus, the compounds which did not melt up to $300{ }^{\circ} \mathrm{C}$ are presented as ">300 ${ }^{\circ} \mathrm{C} "$ after confirming that is not decomposed using NMR). High-resolution mass spectra (HRMS) were obtained by the electron impact (EI) method with a JEOL JMS-T100GCv instrument, by the atmospheric pressure chemical ionization (APCI) method with a BRUKER DALTONICS micrOTOF instrument, and by the electrospray ionization (ESI) method or the direct analysis in real

(1) Still, W. C.; Kahn, M.; Mitra, A. J. Org. Chem. 1978, 43, 2923-2925. 
time (DART) method with IonSense SVP100 and JEOL JMS-T100LP instruments. Low-resolution mass spectra (LRMS) were obtained by the electron impact (EI) method with a Shimadzu GCMSQP2010 Ultra instrument. Purity of isolated compounds was determined by ${ }^{1} \mathrm{H}$ NMR analyses, GC analysis on a Shimadzu GC-2025 instrument equipped with an FID detector and a capillary column (ZB-1MS, Phenomenex, $10 \mathrm{~m} \times 0.10 \mathrm{~mm}$ i.d., $0.10 \mu \mathrm{m}$ film thickness), or HPLC analysis on a JASCO UV-2070 Plus instrument equipped with a reversed-phase C18 column (InertSustain ${ }^{\mathrm{TM}} \mathrm{C} 18$, GL Siences Inc., $4.6 \mathrm{~mm} \times 250 \mathrm{~mm}$ i.d.). Sublimation was conducted by an ULVAC VPC-050/051 instrument $\left(10^{1}-10^{-3} \mathrm{~Pa}\right)$.

Materials. Materials were purchased from Wako Pure Chemical Industries, Ltd. (Wako), Tokyo Chemical Industry Co., Ltd., Aldrich Inc., and other commercial suppliers, and were used after appropriate purification, unless otherwise noted. Florisil (100-200 mesh) was purchased from Kanto Chemical Co., Inc. (Kanto). Boron triiodide was purchased from Aldrich Inc. Arylmagnesium bromides ( $\mathrm{ArMgBr})$ were prepared from the corresponding aryl bromides and magnesium (turnings) using a standard method and titrated before use.

Solvent. Anhydrous solvents were purchased from above-described suppliers and/or dried over Molecular Sieves 4A and degassed before use. Water content of the solvent was determined with a Karl Fischer moisture titrator (AQ-2200, Hiranuma Sangyo Co., Ltd.) to be less than 20 ppm. 
Computational Method. All calculations were performed with performed with Gaussian 09 packages $^{2}$ unless otherwise noted. The DFT method was employed using the B3LYP hybrid functional. ${ }^{3}$ Structures were optimized with the 6-31G(d) basis set. ${ }^{4}$ Structures were optimized with the LANL2DZ ${ }^{4}$ basis set for $\mathrm{Br}$ and $\mathrm{I}$, and $6-31 \mathrm{G}(\mathrm{d})^{5}$ basis set for the other atom types, respectively (denoted as 631LAN). The time-dependent density functional theory (TD-DFT) calculation ${ }^{5}$ was conducted at the B3LYP/6-31G(d) level.

(2) Gaussian 09, Revision C.01, Frisch, M. J.; Trucks, G. W.; Schlegel, H. B.; Scuseria, G. E.; Robb, M. A.; Cheeseman, J. R.; Scalmani, G.; Barone, V.; Mennucci, B.; Petersson, G. A.; Nakatsuji, H.; Caricato, M.; Li, X.; Hratchian, H. P.; Izmaylov, A. F.; Bloino, J.; Zheng, G.; Sonnenberg, J. L.; Hada, M.; Ehara, M.; Toyota, K.; Fukuda, R.; Hasegawa, J.; Ishida, M.; Nakajima, T.; Honda, Y.; Kitao, O.; Nakai, H.; Vreven, T.; Montgomery, Jr., J. A.; Peralta, J. E.; Ogliaro, F.; Bearpark, M.; Heyd, J. J.; Brothers, E.; Kudin, K. N.; Staroverov, V. N.; Keith, R.; Kobayashi, R.; Normand, J.; Raghavachari, K.; Rendell, A.; Burant, J. C.; Iyengar, S. S.; Tomasi, J.; Cossi, M.; Rega, N.; Millam, N. J.; Klene, M.; Knox, J. E.; Cross, J. B.; Bakken, V.; Adamo, C.; Jaramillo, J.; Gomperts, R.; Stratmann, R. E.; Yazyev, O.; Austin, A. J.; Cammi, R.; Pomelli, C.; Ochterski, J. W.; Martin, R. L.; Morokuma, K.; Zakrzewski, V. G.; Voth, G. A.; Salvador, P.; Dannenberg, J. J.; Dapprich, S.; Daniels, A. D.; Farkas, Ö.; Foresman, J. B.; Ortiz, J. V.; Cioslowski, J.; Fox, D. J. Gaussian, Inc., Wallingford CT, 2010.

(3) (a) Becke, A. D. J. Chem. Phys. 1993, 98, 5648-5652. (b) Lee, C.; Yang, W.; Parr, R. G. Phys. Rev. B 1988, 37, 785-789.

(4) Hehre, W. J.; Radom, L.; Schleyer, P. v. R.; Pople, J. A. Ab Initio Molecular Orbital Theory; John Wiley \& Sons: New York, 1986 and references cited therein.

(5) (a) Casida, M. E.; Jamorski, C.; Casida, K. C.; Salahub, D. R. J. Chem. Phys. 1998, 108, 44394449. (b) Stratmann, R. E.; Scuseria, G. E.; Frisch, M. J. J. Chem. Phys. 1998, 109, 8218-8224. 


\section{Summary of DFT calculation}<smiles>c1ccc2c(c1)CCCC2</smiles>

tetralin

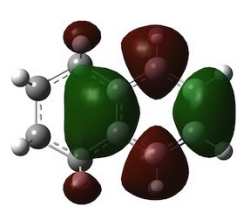

LUMO

$(-0.10 \mathrm{eV})$

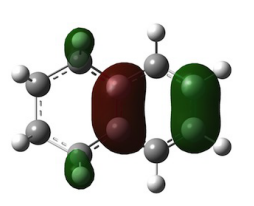

HOMO

(-6.15 eV)<smiles>c1ccc2ccccc2c1</smiles>

naphthalene
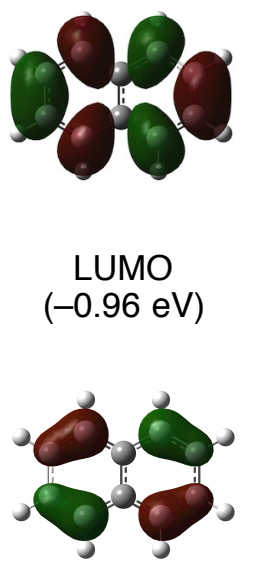

HOMO (-5.79 eV)<smiles>c1ccc2cc3ccccc3cc2c1</smiles>

anthracene

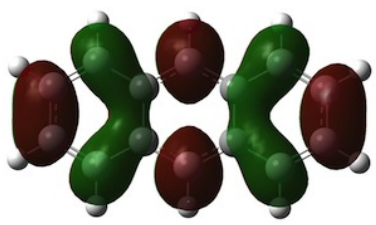

LUMO

$(-1.63 \mathrm{eV})$

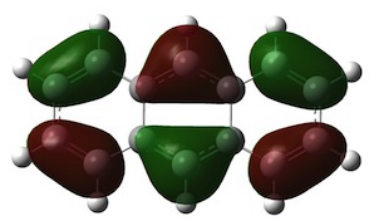

HOMO

$(-5.23 \mathrm{eV})$<smiles>c1ccc(Oc2ccccc2)cc1</smiles>

diphenylether

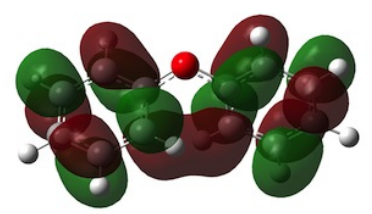

LUMO

$(-0.23 \mathrm{eV})$

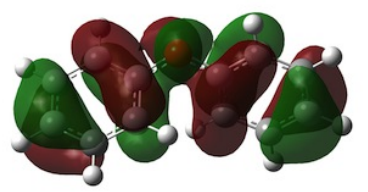

HOMO

$(-5.89 \mathrm{eV})$<smiles>c1ccc2c(c1)Cc1ccccc1-2</smiles>

fluorene

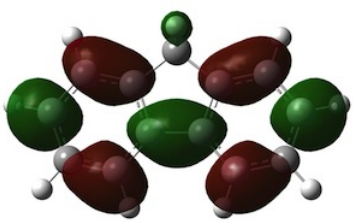

LUMO

$(-0.71 \mathrm{eV})$

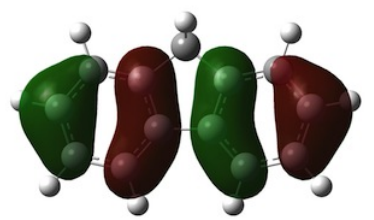

HOMO

$(-5.75 \mathrm{eV})$

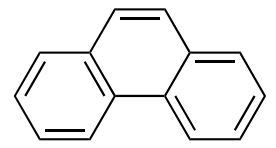

phenanthrene

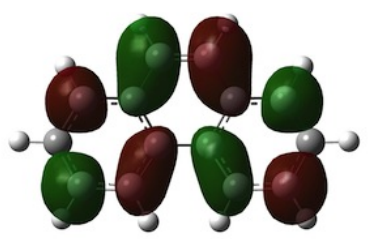

LUMO

$(-0.99 \mathrm{eV})$

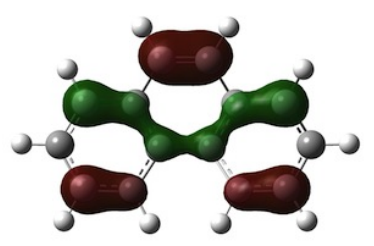

HOMO

$(-5.73 \mathrm{eV})$<smiles>Cc1cccc(Cl)c1C</smiles>

3-chloroo-xylene

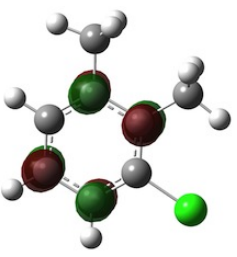

LUMO $(-0.19 \mathrm{eV})$

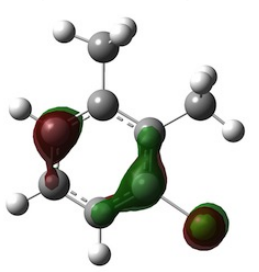

HOMO

(-6.49 eV)

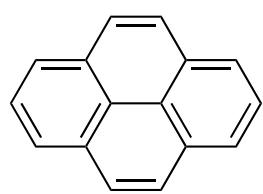

pyrene

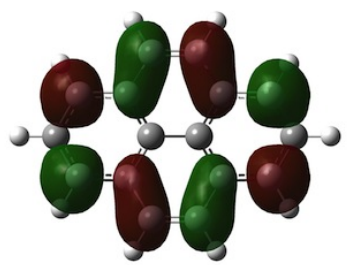

LUMO

$(-1.48 \mathrm{eV})$

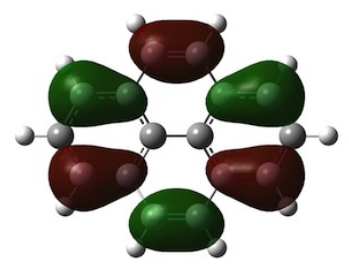

HOMO $(-5.33 \mathrm{eV})$

Figure S1. Kohn-Sham frontier orbitals of tetralin, naphthalene, anthracene, diphenylether, fluorene, phenanthrene, 3-chloro-o-xylene and pyrene calculated at the B3LYP/6-31G(d) level of theory. 


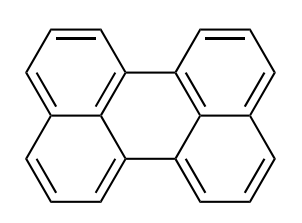

perylene

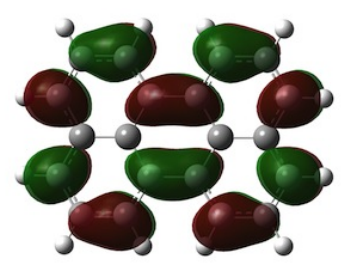

LUMO

(-1.89 eV)

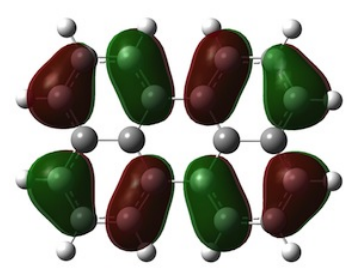

HOMO

(-4.95 eV)

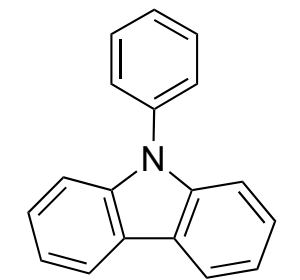

9-phenylcarbazole

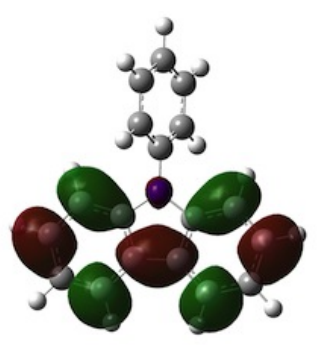

LUMO

$(-0.65 \mathrm{eV})$

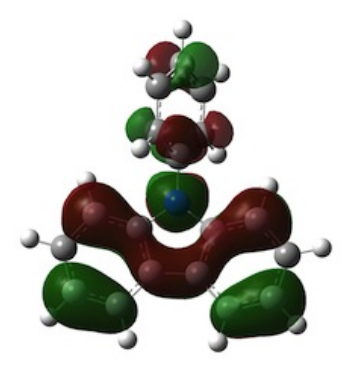

HOMO

(-5.33 eV)

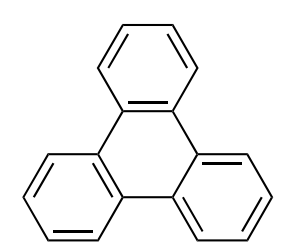

triphenylene
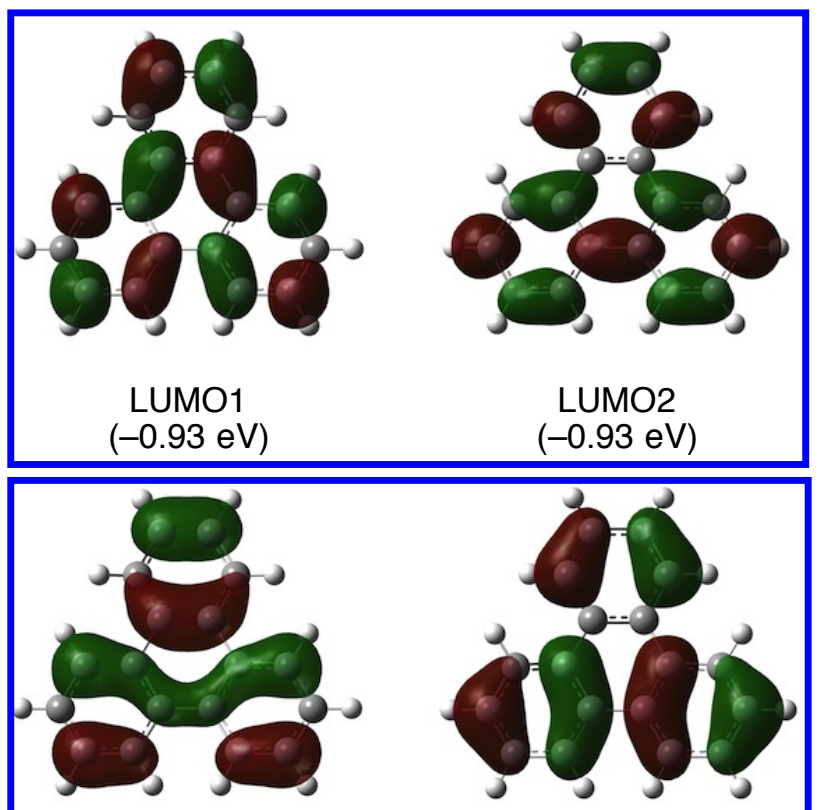

HOMO1

$(-5.85 \mathrm{eV})$

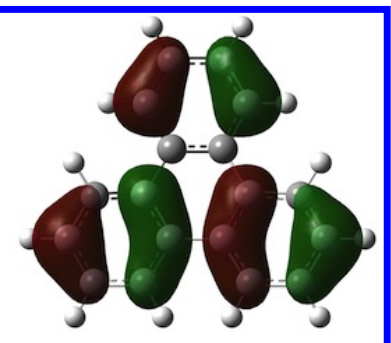

HOMO2

$(-5.85 \mathrm{eV})$

Figure S2. Kohn-Sham frontier orbitals of perylene, 9-phenylcarbazole and triphenylene calculated at the B3LYP/6-31G(d) level of theory. Degenerate orbitals are shown in a blue square. 
<smiles>c1ccc(-c2c3ccccc3c(-c3ccccc3)c3ccccc23)cc1</smiles>

9,10-diphenylanthracene<smiles>Cc1cccc(-c2cccc(C)c2)c1</smiles>

3,3'-dimethylbiphenyl

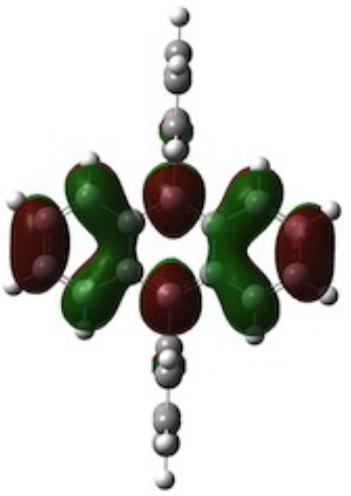

LUMO $(-1.60 \mathrm{eV})$

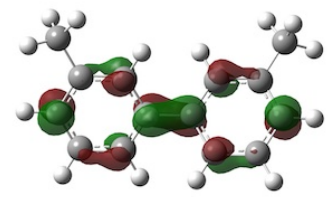

$$
\begin{aligned}
& \text { LUMO } \\
& (-0.59 \mathrm{eV})
\end{aligned}
$$

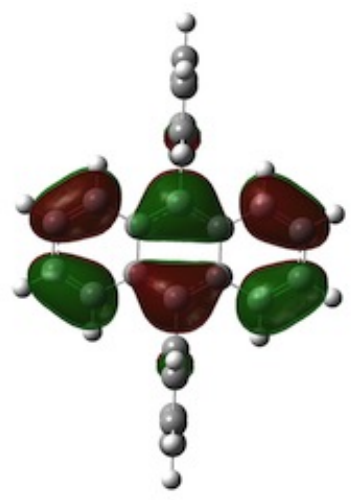

HOMO $(-5.10 \mathrm{eV})$

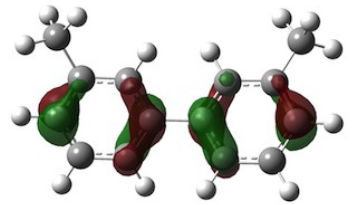

$$
\text { HOMO }
$$$$
(-5.91 \mathrm{eV})
$$

Figure S3. Kohn-Sham frontier orbitals of 9,10-diphenylanthracene and 3,3'-dimethylbiphenyl calculated at the B3LYP/6-31G(d) level of theory. 
Table S1. Electronic Energies $(E)$, Relative Energies $(\Delta E)$ and Gibbs Free Energies $(G$ : sum of Electronic and Thermal Free Energies) of Stationary Points (in hartrees) Calculated at the B3LYP/631LAN Level of Theory.

\begin{tabular}{|c|c|c|c|c|}
\hline compounds & $E($ A.U. $)$ & $\Delta E(\mathrm{kcal} / \mathrm{mol})$ & $G$ (A.U.) & $\Delta G(\mathrm{kcal} / \mathrm{mol})$ \\
\hline tetralin-5- $\mathrm{BI}_{2}$ & -435.3800 & +6.82 & -435.2336 & +6.84 \\
\hline tetralin-6- $-\mathrm{BI}_{2}$ & -435.3908 & 0 & -435.2445 & 0 \\
\hline 3-chloro-o-xylene-4-BI ${ }_{2}$ & -817.5515 & +5.95 & -817.4557 & +5.09 \\
\hline 3-chloro-o-xylene-5- $\mathrm{BI}_{2}$ & -817.5610 & 0 & -817.4638 & 0 \\
\hline 3-chloro-o-xylene-6- $\mathrm{BI}_{2}$ & -817.5507 & +6.49 & -817.4529 & +6.84 \\
\hline naphthalene-1-BI ${ }_{2}$ & -432.9665 & +5.80 & -432.8656 & +5.67 \\
\hline naphthalene-2- $\mathrm{BI}_{2}$ & -432.9758 & 0 & -432.8746 & 0 \\
\hline anthracene-1- $\mathrm{BI}_{2}$ & -586.6049 & +5.72 & -586.4605 & +5.75 \\
\hline anthracene-2- $\mathrm{BI}_{2}$ & -586.6141 & 0 & -586.4697 & 0 \\
\hline anthracene-9- $\mathrm{BI}_{2}$ & -586.6006 & +8.43 & -586.4564 & +8.36 \\
\hline $\mathrm{Ph}_{2}$-anthracene-1- $\mathrm{BI}_{2}$ & -1048.6957 & +11.7 & -1048.4030 & +13.4 \\
\hline $\mathrm{Ph}_{2}$-anthracene-2- $\mathrm{BI}_{2}$ & -1048.7144 & 0 & -1048.4242 & +0.167 \\
\hline $\mathrm{Ph}_{2}$-anthracene-9a- $\mathrm{BI}_{2}$ & -1048.7030 & +7.12 & -1048.4107 & +8.63 \\
\hline $\mathrm{Ph}_{2}$-anthracene- $9 \mathrm{~b}-\mathrm{BI}_{2}$ & -1048.7125 & +1.19 & -1048.4245 & 0 \\
\hline $\mathrm{Ph}_{2}$-anthracene-9c-BI & -1048.7133 & +0.654 & -1048.4231 & +0.839 \\
\hline triphenylene-1- $\mathrm{BI}_{2}$ & -740.2491 & +9.72 & -740.0614 & +9.83 \\
\hline triphenylene-2- $\mathrm{BI}_{2}$ & -740.2646 & 0 & -740.0771 & 0 \\
\hline diphenylether-2- $-\mathrm{BI}_{2}$ & -585.5903 & +5.24 & -585.4570 & +5.68 \\
\hline diphenylether-3- $\mathrm{BI}_{2}$ & -585.5941 & +2.82 & -585.4624 & +2.28 \\
\hline diphenylether-4- $\mathrm{BI}_{2}$ & -585.5986 & 0 & -585.4661 & 0 \\
\hline fluorene-1- $\mathrm{BI}_{2}$ & -548.5001 & +4.86 & -548.3611 & +4.73 \\
\hline fluorene-2- $\mathrm{BI}_{2}$ & -548.5078 & 0 & -548.3687 & 0 \\
\hline fluorene-3-BI 2 & -548.5063 & +0.966 & -548.3673 & +0.880 \\
\hline fluorene-4- $\mathrm{BI}_{2}$ & -548.4954 & +7.80 & -548.3573 & +7.11 \\
\hline phenanthrene-1- $\mathrm{BI}_{2}$ & -586.6115 & +6.68 & -586.4672 & +6.43 \\
\hline phenanthrene-2- $\mathrm{BI}_{2}$ & -586.6219 & +0.165 & -586.4773 & +0.114 \\
\hline phenanthrene-3- $\mathrm{BI}_{2}$ & -586.6222 & 0 & -586.4775 & 0 \\
\hline phenanthrene-4- $\mathrm{BI}_{2}$ & -586.6066 & +9.77 & -586.4621 & +9.61 \\
\hline phenanthrene-9- $\mathrm{BI}_{2}$ & -586.6122 & +6.29 & -586.4677 & +6.13 \\
\hline 9-phenylcarbazole-1- $\mathrm{BI}_{2}$ & -795.5935 & +9.27 & -795.3910 & +9.78 \\
\hline 9-phenylcarbazole-2- $\mathrm{BI}_{2}$ & -795.6059 & +1.47 & -795.4040 & +1.61 \\
\hline 9-phenylcarbazole-3- $\mathrm{BI}_{2}$ & -795.6083 & 0 & -795.4066 & 0 \\
\hline 9-phenylcarbazole-4- $\mathrm{BI}_{2}$ & -795.5950 & +8.33 & -795.3934 & +8.25 \\
\hline 9-phenylcarbazole-9a-BI ${ }_{2}$ & -795.5938 & +9.06 & -795.3919 & +9.23 \\
\hline 9-phenylcarbazole-9b-BI ${ }_{2}$ & -795.6016 & +4.18 & -795.4003 & +3.97 \\
\hline 9-phenylcarbazole-9c-- $\mathrm{BI}_{2}$ & -795.6041 & +2.60 & -795.4013 & +3.31 \\
\hline pyrene-1-BI ${ }_{2}$ & -662.8476 & +4.80 & -662.6906 & +4.91 \\
\hline pyrene-2- $\mathrm{BI}_{2}$ & -662.8553 & 0 & -662.6984 & 0 \\
\hline pyrene-4-BI 2 & -662.8470 & +5.22 & -662.6899 & +5.31 \\
\hline perylene-1-BI 2 & -816.4764 & +7.57 & -816.2760 & +8.26 \\
\hline perylene-2- $\mathrm{BI}_{2}$ & -816.4885 & 0 & -816.2892 & 0 \\
\hline perylene-3- $\mathrm{BI}_{2}$ & -816.4810 & +4.71 & -816.2813 & +4.93 \\
\hline 3,3'-dimethylbiphenyl-2-BI ${ }_{2}$ & -588.9992 & +8.46 & -588.8282 & +9.90 \\
\hline 3,3'-dimethylbiphenyl-4-BI & -589.0054 & +4.58 & -588.8346 & +5.87 \\
\hline 3,3'-dimethylbiphenyl-5- $\mathrm{BI}_{2}$ & -589.0127 & 0 & -588.8440 & 0 \\
\hline 3,3'-dimethylbiphenyl-6- $\mathrm{BI}_{2}$ & -589.0043 & +5.24 & -588.8348 & +5.76 \\
\hline
\end{tabular}


Scheme S1. Electrophilic C-H Borylation of 3-Chloro-o-xylene Using Equimolar or Excess Boron Triiodide

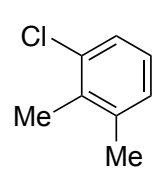

$1 \mathrm{~b}$

(1.0 equiv)

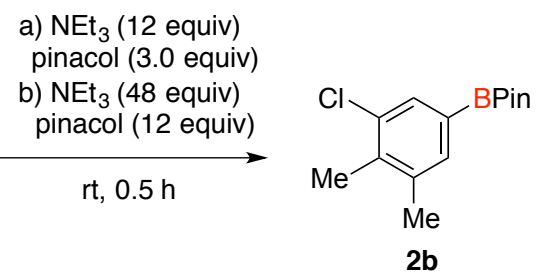

a) $4 \%(\mathrm{NMR})$

b) $25 \%(\mathrm{NMR})$

Table S2. Screening of Reaction Conditions for Borylation of Naphthalene.

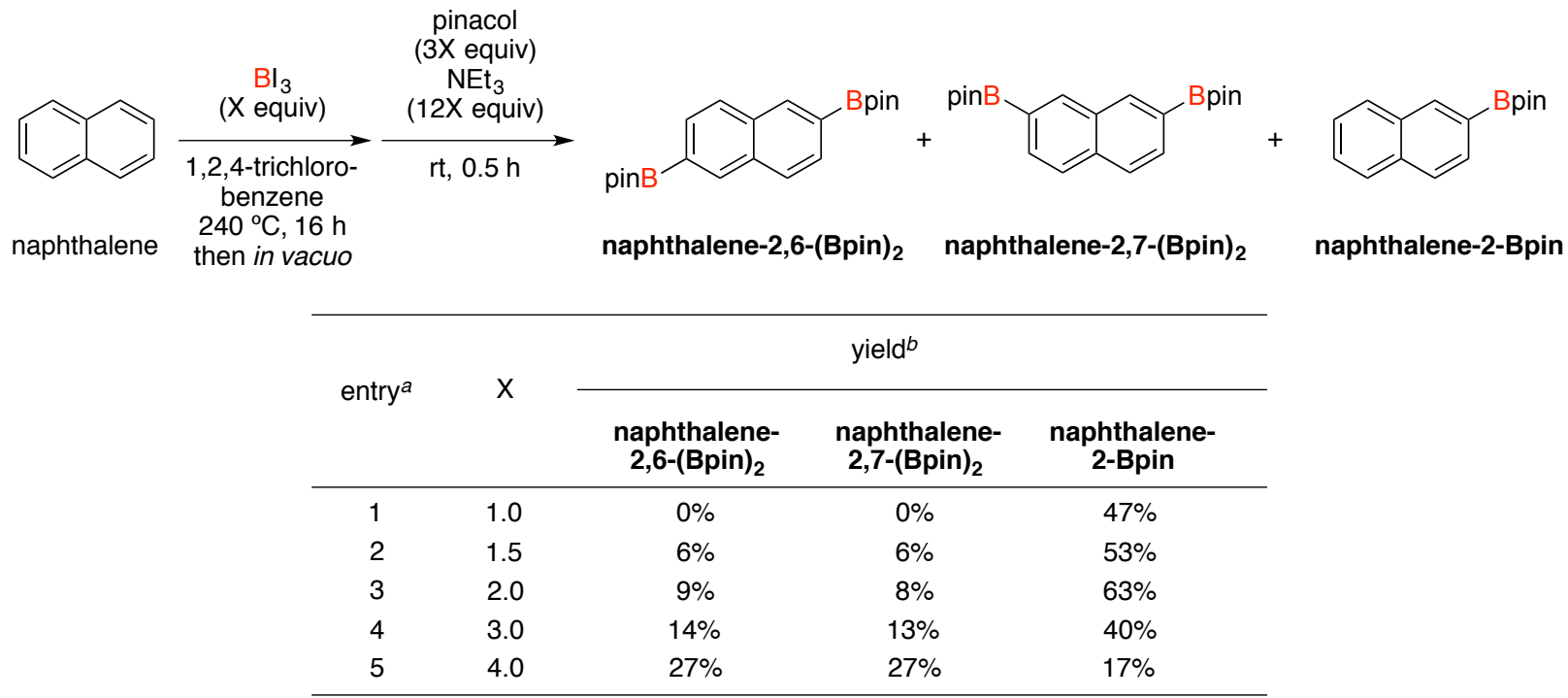

aReactions were carried out on a $0.2 \mathrm{mmol}$ scale. ${ }^{b}$ Yields were determined by ${ }^{1} \mathrm{H}$ NMR analysis using 1,1,2,2-tetrachloroethane as an internal standard. 


\section{Synthesis of boron triiodide $\left(\mathrm{BI}_{3}\right)^{6}$}

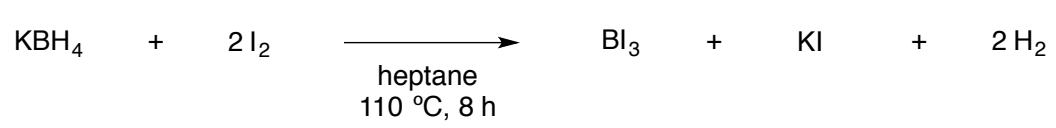

To a $500 \mathrm{~mL}$ three necked round-bottomed flask equipped with a reflux condenser were added potassium borohydride $(14.6 \mathrm{~g}, 270 \mathrm{mmol})$, iodine $(45.7 \mathrm{~g}, 180 \mathrm{mmol})$ and heptane $(60 \mathrm{~mL})$ at room temperature under a nitrogen atmosphere. After stirring at $110^{\circ} \mathrm{C}$ for $8 \mathrm{~h}$, the reaction mixture was allowed to cool to room temperature and then heptane was removed in vacuo. The crude product was purified by sublimation using a sublimation apparatus to obtain title compound (15.5 g) as a white solid. ${ }^{11} \mathrm{~B}$ NMR $\left(129 \mathrm{MHz}, \mathrm{CDCl}_{3}\right) \delta-8.27$.

\section{Synthesis of 4,4,5,5-tetramethyl-2-(5,6,7,8-tetrahydro-2-naphthalenyl)-1,3,2-dioxaborolane}

\section{(2a: tetralin-6-Bpin)}

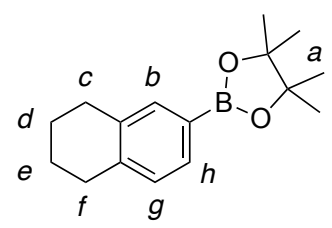

To a Schlenk tube were added boron triiodide $(0.392 \mathrm{~g}, 1.0 \mathrm{mmol})$ and 1,2,3,4-tetrahydronaphthalene $(2.04 \mathrm{~mL}, 15 \mathrm{mmol})$ at room temperature under a nitrogen atmosphere. After stirring at $160^{\circ} \mathrm{C}$ for 16 $\mathrm{h}$, the reaction mixture was allowed to cool to room temperature and then hydrogen iodide was removed in vacuo. Triethylamine $(1.66 \mathrm{~mL}, 12 \mathrm{mmol})$ and pinacol $(0.355 \mathrm{~g}, 3.0 \mathrm{mmol})$ were added to the reaction mixture at $0{ }^{\circ} \mathrm{C}$ through a cannula. After stirring at room temperature for $0.5 \mathrm{~h}$, the solvent was removed in vacuo. The yield of the title compound in the crude product was determined to be $94 \%$ by ${ }^{1} \mathrm{H}$ NMR using 1,1,2,2-tetrachloroethane as an internal standard. The crude product was purified by GPC (eluent: toluene) to obtain the title compound $(0.217 \mathrm{~g}, 84 \%$ yield, $>99 \%$ pure on ${ }^{1} \mathrm{H}$ NMR analysis) as a yellow liquid. IR (neat): $\mathrm{cm}^{-1}$ 2976, 2926, 2857 (Ar-H), 2837 (Ar-H), 1612, 1566, 1506, 1435, 1406, 1387, 1379, 1362, 1348, 1327, 1310, 1273, 1215, 1182, 1165, 1144, 1128, $1111,1090,1063,989,962,922,885,862,851,814,775,733,716,667 ;{ }^{1} \mathrm{H} \mathrm{NMR}\left(400 \mathrm{MHz}, \mathrm{CDCl}_{3}\right)$ $\delta 1.33(\mathrm{~s}, 12 \mathrm{H}, a), 1.78-1.80(\mathrm{~m}, 4 \mathrm{H}, d, e), 2.78-2.79(\mathrm{~m}, 4 \mathrm{H}, c, f), 7.07(\mathrm{~d}, J=8.2 \mathrm{~Hz}, 1 \mathrm{H}, g), 7.52$ $(\mathrm{d}, J=8.2 \mathrm{~Hz}, 1 \mathrm{H}, h), 7.53(\mathrm{~s}, 1 \mathrm{H}, b) ;{ }^{13} \mathrm{C} \mathrm{NMR}\left(101 \mathrm{MHz}, \mathrm{CDCl}_{3}\right) \delta 23.1(1 \mathrm{C}), 23.2(1 \mathrm{C}), 24.8(4 \mathrm{C})$, 29.1 (1C), 29.6 (1C), 83.5 (2C), 128.6 (1C), 131.7 (1C), 135.7 (1C), 136.5 (1C), 140.7 (1C). The NMR signal of the carbon $\alpha$ to the boron was not observed.; ${ }^{11} \mathrm{~B}$ NMR $\left(129 \mathrm{MHz}, \mathrm{CDCl}_{3}\right) \delta 30.5$;

(6) (a) Briggs, A. G. Naturwissenschaften 1990, 77, 595. (b) Mercier, H. P. A.; Moran, M. D.; Schrobilgen, G. J.; Steinberg, C.; Suontamo, R. J. J. Am. Chem. Soc. 2004, 126, 5533. 
Synthesis of 2-(3-chloro-4,5-dimethylphenyl)-4,4,5,5-tetramethyl-1,3,2-dioxaborolane (2b: 3chloro-o-xylene-5-Bpin)

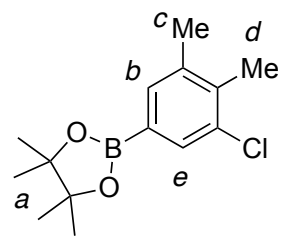

To a Schlenk tube were added boron triiodide $(0.392 \mathrm{~g}, 1.0 \mathrm{mmol})$ and 3-chloro- $o$-xylene $(1.95 \mathrm{~mL}$, $15 \mathrm{mmol}$ ) at room temperature under a nitrogen atmosphere. After stirring at $160{ }^{\circ} \mathrm{C}$ for $16 \mathrm{~h}$, the reaction mixture was allowed to cool to room temperature and then hydrogen iodide was removed in vacuo. Triethylamine $(1.65 \mathrm{~mL}, 12 \mathrm{mmol})$ and pinacol $(0.356 \mathrm{~g}, 3.0 \mathrm{mmol})$ were added to the reaction mixture at room temperature. After stirring at room temperature for $0.5 \mathrm{~h}$, the solvent was removed in vacuo. The yield of the title compound in the crude product was determined to be $76 \%$ by ${ }^{1} \mathrm{H} \mathrm{NMR}$ using 1,1,2,2-tetrachloroethane as an internal standard. The crude product was purified by GPC (eluent: toluene) to obtain the title compound ( $0.176 \mathrm{~g}, 66 \%$ yield, $>99 \%$ pure on ${ }^{1} \mathrm{H}$ NMR analysis) as a yellow solid. IR (neat): $\mathrm{cm}^{-1} 2976,2967,2930,1607,1549,1483,1389,1371,1358,1346,1315$, $1271,1248,1215,1167,1148,1126,1107,1009,995,966,916,885,854,839,826,775,759,731$, 719, 712, 691, 677, 651; mp: 115.0-115.3 ${ }^{\circ} \mathrm{C} ;{ }^{1} \mathrm{H}$ NMR (400 MHz, $\left.\mathrm{CDCl}_{3}\right) \delta 1.33(\mathrm{~s}, 12 \mathrm{H}, a), 2.30$ $(\mathrm{s}, 3 \mathrm{H}, c), 2.33(\mathrm{~s}, 3 \mathrm{H}, d), 7.46(\mathrm{~s}, 1 \mathrm{H}, b), 7.64(\mathrm{~s}, 1 \mathrm{H}, e) ;{ }^{13} \mathrm{C} \mathrm{NMR}\left(126 \mathrm{MHz}, \mathrm{CDCl}_{3}\right) \delta 16.5(1 \mathrm{C})$, 20.6 (1C), 24.8 (4C), 83.9 (2C), 132.9 (1C), 134.2 (1C), 134.5 (1C), 137.7 (1C), 137.9 (1C). The NMR signal of the carbon $\alpha$ to the boron was not observed.; ${ }^{11} \mathrm{~B}$ NMR $\left(129 \mathrm{MHz}, \mathrm{CDCl}_{3}\right) \delta 29.6$; HRMS (ESI-) $m / z$ [M-H] $]^{-}$calcd for $\mathrm{C}_{14} \mathrm{H}_{19} \mathrm{BClO}_{2} 265.1169$; observed 265.1195 .

\section{Synthesis of 4,4,5,5-tetramethyl-2-(2-naphthalenyl)-1,3,2-dioxaborolane (2c: naphthalene-2-}

\section{Bpin)}

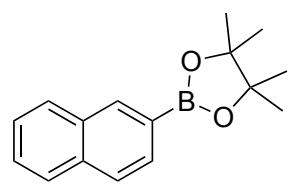

To a Schlenk tube were added boron triiodide $(77.8 \mathrm{mg}, 0.20 \mathrm{mmol})$, naphthalene $(0.103 \mathrm{~g}, 0.80$ $\mathrm{mmol})$ and 1,2,4-trichlorobenzene $(1.6 \mathrm{~mL})$ at room temperature under a nitrogen atmosphere. After stirring at $180{ }^{\circ} \mathrm{C}$ for $16 \mathrm{~h}$, the reaction mixture was allowed to cool to room temperature and then hydrogen iodide was removed in vacuo. Triethylamine $(0.340 \mathrm{~mL}, 2.4 \mathrm{mmol})$ and pinacol $(72.1 \mathrm{mg}$, $0.61 \mathrm{mmol}$ ) were added to the reaction mixture at room temperature. After stirring at room temperature for $0.5 \mathrm{~h}$, the solvent was removed in vacuo $\left(100^{\circ} \mathrm{C}, 0.23 \mathrm{mmHg}\right)$. The yield of the title compound in the crude product was determined to be $62 \%$ by ${ }^{1} \mathrm{H}$ NMR using $1,1,2,2-$ 
tetrachloroethane as an internal standard. The crude product was purified by GPC (eluent: toluene) to obtain the title compound (27.1 mg, 53\% yield, $>99 \%$ pure on ${ }^{1} \mathrm{H}$ NMR analysis) as a yellow solid. The compound was identified in comparison with the reported data ${ }^{7}$.

Synthesis of 2-(2-anthracenyl)-4,4,5,5-tetramethyl-1,3,2-dioxaborolane (2d: anthracene-2Bpin)

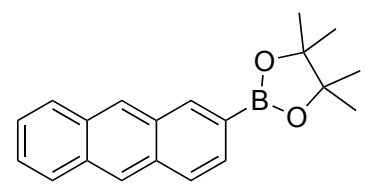

To a Schlenk tube were added boron triiodide $(78.1 \mathrm{mg}, 0.20 \mathrm{mmol})$, anthracene $(0.143 \mathrm{~g}, 0.80 \mathrm{mmol})$ and 1,2,4-trichlorobenzene $(1.6 \mathrm{~mL})$ at room temperature under a nitrogen atmosphere. After stirring at $180{ }^{\circ} \mathrm{C}$ for $4 \mathrm{~h}$, the reaction mixture was allowed to cool to room temperature and then hydrogen iodide was removed in vacuo. Triethylamine $(0.340 \mathrm{~mL}, 2.4 \mathrm{mmol})$ and pinacol $(70.8 \mathrm{mg}, 0.60 \mathrm{mmol})$ were added to the reaction mixture at room temperature. After stirring at room temperature for $0.5 \mathrm{~h}$, the solvent was removed in vacuo $\left(100^{\circ} \mathrm{C}, 0.23 \mathrm{mmHg}\right)$. The yield of the title compound in the crude product was determined to be $70 \%$ by ${ }^{1} \mathrm{H}$ NMR using 1,1,2,2-tetrachloroethane as an internal standard. The crude product was purified by GPC (eluent: toluene) to obtain the title compound (33.5 $\mathrm{mg}, 55 \%$ yield, $>99 \%$ pure on ${ }^{1} \mathrm{H}$ NMR analysis) as a yellow solid. The compound was identified in comparison with the reported data ${ }^{8}$.

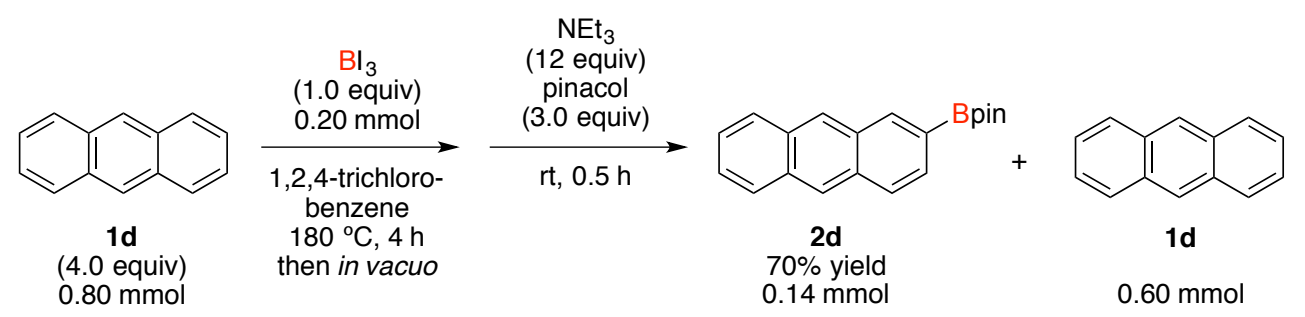

Synthesis of 2-(9,10-diphenyl-2-anthracenyl)-4,4,5,5-tetramethyl-1,3,2-dioxaborolane (2e: $\mathrm{Ph}_{2-}$ anthracene-2-Bpin)

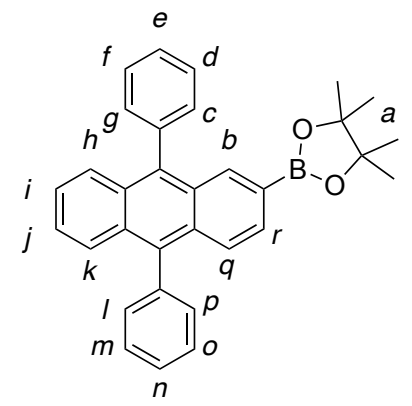

(6) Kinuta, H.; Tobisu, M.; Chatani, N. J. Am. Chem. Soc. 2015, 137, 1593.

(7) Yoshida, T.; Ilies, L.; Nakamura, E. ACS. Catal. 2017, 7, 3199. 
To a Schlenk tube were added boron triiodide (78.4 mg, $0.20 \mathrm{mmol}), 9,10$-diphenylanthracene $(0.265$ g, $0.80 \mathrm{mmol})$ and 1,2,4-trichlorobenzene $(1.6 \mathrm{~mL})$ at room temperature under a nitrogen atmosphere. After stirring at $180{ }^{\circ} \mathrm{C}$ for $2 \mathrm{~h}$, the reaction mixture was allowed to cool to room temperature and then hydrogen iodide was removed in vacuo. Triethylamine $(0.340 \mathrm{~mL}, 2.4 \mathrm{mmol})$ and pinacol $(71.8$ $\mathrm{mg}, 0.61 \mathrm{mmol}$ ) were added to the reaction mixture at room temperature. After stirring for $0.5 \mathrm{~h}$, the solvent was removed in vacuo $\left(100{ }^{\circ} \mathrm{C}, 0.23 \mathrm{mmHg}\right)$. The yield of the title compound in the crude product was determined to be $70 \%$ by ${ }^{1} \mathrm{H}$ NMR using 1,1,2,2-tetrachloroethane as an internal standard. The crude product was purified by GPC (eluent: toluene) to obtain the title compound (51.1 mg, 56\% yield, $>98 \%$ pure on ${ }^{1} \mathrm{H}$ NMR analysis) as a yellow solid. IR (neat): $\mathrm{cm}^{-1} 3026$ (Ar-H), 2981, 2358, 2322, 1716, 1683, 1620, 1610, 1575, 1558, 1496, 1479, 1458, 1440, 1419, 1392, 1371, 1354, 1338, 1309, 1269, 1213, 1143, 1134, 1116, 1080, 1024, 1001, 974, 945, 914, 883, 854, 827, 798, 759, 750, 734, 696, 665, 630, 623, 615; mp: 238.1-240.5 ${ }^{\circ} \mathrm{C} ;{ }^{1} \mathrm{H}$ NMR (500 MHz, $\left.\mathrm{CDCl}_{3}\right) \delta 1.29$ (s, 12H,a), 7.30-7.35 (m, 2H, $i, j), 7.46-7.51(\mathrm{~m}, 4 \mathrm{H}, c, g, l, p), 7.53-7.68(\mathrm{~m}, 10 \mathrm{H}, d, e, f, h, k, m, n, o, q, r)$, $8.25(\mathrm{~s}, 1 \mathrm{H}, b) ;{ }^{13} \mathrm{C} \mathrm{NMR}\left(126 \mathrm{MHz}, \mathrm{CDCl}_{3}\right) \delta 24.8(4 \mathrm{C}), 83.7$ (2C), $124.9(1 \mathrm{C}), 125.4(1 \mathrm{C}), 125.8$ (1C), 126.9 (1C), 127.2 (1C), 127.4 (1C), 127.5 (1C), 128.3 (2C), 128.4 (2C), 129.0 (1C), 129.2 (1C), 130.0 (1C), 130.5 (1C), 130.9 (1C), 131.3 (2C), 131.5 (2C), 135.6 (1C), 136.8 (1C), 138.3 (1C), 138.7 (1C), 139.1 (1C). The NMR signal of the carbon $\alpha$ to the boron was not observed.; ${ }^{11} \mathrm{~B}$ NMR (129 $\left.\mathrm{MHz}, \mathrm{CDCl}_{3}\right) \delta 30.6$; HRMS (ESI+) $m / z[\mathrm{M}]^{+}$calcd for $\mathrm{C}_{32} \mathrm{H}_{29} \mathrm{BO}_{2} 456.2261$; observed 456.2294.

\section{Synthesis of 4,4,5,5-tetramethyl-2-(2-phenanthrenyl)-1,3,2-dioxaborolaneand 4,4,5,5-}

\section{tetramethyl-2-(3-phenanthrenyl)-1,3,2-dioxaborolane (2f: phenanthrene -2-Bpin}

\section{phenanthrene -3-Bpin)}

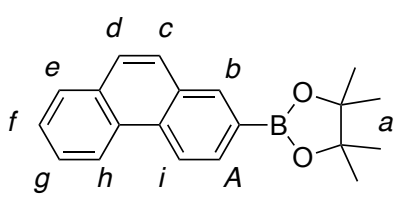

phenanthrene-2-Bpin

51

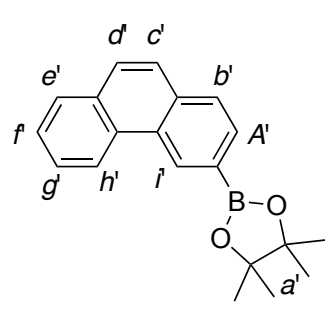

phenanthrene-3-Bpin

49

To a Schlenk tube were added boron triiodide $(0.156 \mathrm{~g}, 0.40 \mathrm{mmol})$, phenanthrene $(0.287 \mathrm{~g}, 1.6 \mathrm{mmol})$ and 1,2,4-trichlorobenzene $(1.6 \mathrm{~mL})$ at room temperature under a nitrogen atmosphere. After stirring at $180^{\circ} \mathrm{C}$ for $12 \mathrm{~h}$, the reaction mixture was allowed to cool to room temperature and then hydrogen iodide was removed in vacuo. Triethylamine $(0.670 \mathrm{~mL}, 4.8 \mathrm{mmol})$ and pinacol $(0.142 \mathrm{~g}, 1.2 \mathrm{mmol})$ were added to the reaction mixture at room temperature. After stirring at room temperature for $0.5 \mathrm{~h}$, the solvent was removed in vacuo $\left(100{ }^{\circ} \mathrm{C}, 0.23 \mathrm{mmHg}\right)$. The yields of the title compounds in the crude product were determined to be $37 \%$ and $37 \%$ (total yield: $74 \%$ ) by ${ }^{1} \mathrm{H}$ NMR using 1,1,2,2tetrachloroethane as an internal standard. The crude product was purified by GPC (eluent: toluene) 
to obtain a 51:49 mixture of phenanthrene-2-Bpin and phenanthrene-3-Bpin $(73.3 \mathrm{mg}, 60 \%$ yield, $>98 \%$ pure on ${ }^{1} \mathrm{H}$ NMR analysis) as a yellow liquid. IR (neat): $\mathrm{cm}^{-1}$ 2978, 2929 (Ar-H), 1737, 1616, 1598, 1568, 1527, 1514, 1469, 1456, 1423, 1398, 1363, 1338, 1305, 1294, 1269, 1242, 1215, 1165 , 1139, 1101, 1085, 1024, 1001, 962, 910, 877, 866, 850, 829, 812, 746, 719, 692, 677, 632, 624, 605; ${ }^{1} \mathrm{H}$ NMR (400 MHz, $\left.\mathrm{CDCl}_{3}\right) \delta 1.39(\mathrm{~s}, 12 \mathrm{H}, a), 1.40\left(\mathrm{~s}, 12 \mathrm{H}, a^{\prime}\right), 7.55-7.66\left(\mathrm{~m}, 4 \mathrm{H}, f, f^{\prime}, g, g^{\prime}\right), 7.70-$ $7.78\left(\mathrm{~m}, 4 \mathrm{H}, c, c^{\prime}, d, d^{\prime}\right), 7.85-7.87\left(\mathrm{~m}, 3 \mathrm{H}, b^{\prime}, e, e^{\prime}\right), 8.00\left(\mathrm{dd}, J=0.9,7.8 \mathrm{~Hz}, 1 \mathrm{H}, A^{\prime}\right), 8.05(\mathrm{dd}, J=$ $0.9,7.8 \mathrm{~Hz}, 1 \mathrm{H}, A), 8.39(\mathrm{~s}, 1 \mathrm{H}, b), 8.66\left(\mathrm{~d}, J=8.7 \mathrm{~Hz}, 1 \mathrm{H}, h\right.$ or $h^{\prime}$ or $\left.i\right), 8.69(\mathrm{~d}, J=7.8 \mathrm{~Hz}, 1 \mathrm{H}, h$ or $h^{\prime}$ or $\left.i\right), 8.84\left(\mathrm{~d}, J=7.8 \mathrm{~Hz}, 1 \mathrm{H}, h\right.$ or $h^{\prime}$ or $\left.i\right), 9.20\left(\mathrm{~s}, 1 \mathrm{H}, i^{\prime}\right) ;{ }^{13} \mathrm{C} \mathrm{NMR}\left(101 \mathrm{MHz}, \mathrm{CDCl}_{3}\right) \delta 24.9(8 \mathrm{C})$, 83.9 (4C), 121.8 (1C), 123.0 (2C), 126.5 (2C), 126.6 (1C), 126.8 (2C), 127.0 (1C), 127.3 (1C), 127.7 (1C), 128.1 (1C), 128.5 (2C), 129.5 (1C), 130.1 (1C), 130.5 (1C), 131.3 (1C), 131.8 (2C), 132.0 (1C), 132.3 (1C), 132.5 (1C), 134.0 (1C), 136.2 (2C). The NMR signal of the carbon $\alpha$ to the boron was not observed.; ${ }^{11} \mathrm{~B}$ NMR $\left(129 \mathrm{MHz}, \mathrm{CDCl}_{3}\right) \delta 30.5$; HRMS (ESI+) $m / z[\mathrm{M}+\mathrm{H}]^{+}$calcd for $\mathrm{C}_{20} \mathrm{H}_{22} \mathrm{BO}_{2}$ 305.1716; observed 305.1731 .

\section{Synthesis of 2-(9H-fluoren-2-yl)-4,4,5,5-tetramethyl-1,3,2-dioxaborolane and 3-(9H-fluoren-2-} yl)-4,4,5,5-tetramethyl-1,3,2-dioxaborolane (2g: fluorene-2-Bpin and fluorene-3-Bpin)

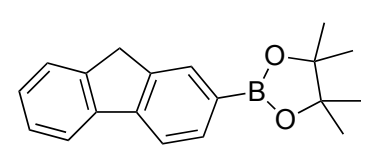

fluorene-2-Bpin

63

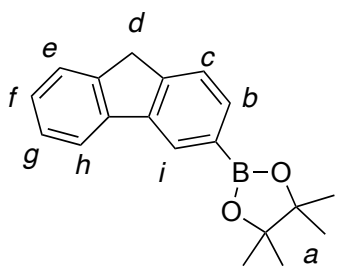

fluorene-3-Bpin

37

To a Schlenk tube were added boron triiodide $(0.159 \mathrm{~g}, 0.40 \mathrm{mmol})$, fluorene $(0.266 \mathrm{~g}, 1.6 \mathrm{mmol})$ and 1,2,4-trichlorobenzene $(1.6 \mathrm{~mL})$ at room temperature under a nitrogen atmosphere. After stirring at $180^{\circ} \mathrm{C}$ for $12 \mathrm{~h}$, the reaction mixture was allowed to cool to room temperature and then hydrogen iodide was removed in vacuo. Triethylamine $(0.670 \mathrm{~mL}, 4.8 \mathrm{mmol})$ and pinacol $(0.147 \mathrm{~g}, 1.2 \mathrm{mmol})$ were added to the reaction mixture at room temperature. After stirring at room temperature for $0.5 \mathrm{~h}$, the solvent was removed in vacuo $\left(100{ }^{\circ} \mathrm{C}, 0.23 \mathrm{mmHg}\right)$. The yields of the title compounds in the crude product were determined to be $38 \%$ and $26 \%$ (total yield: $64 \%$ ) by ${ }^{1} \mathrm{H}$ NMR using 1,1,2,2tetrachloroethane as an internal standard. The crude product was purified by GPC (eluent: toluene) to obtain a 63:37 mixture of fluorene-2-Bpin and fluorene-3-Bpin (56.1 mg, 48\% yield, >98\% pure on ${ }^{1} \mathrm{H}$ NMR analysis) as a yellow liquid. IR (neat): $\mathrm{cm}^{-1}$ 2976, 2927 (Ar-H), 1737, 1612, 1570, 1490, $1458,1417,1379,1371,1352,1327,1315,1263,1230,1213,1184,1165,1141,1101,1076,1045$, $1031,1002,962,950,923,896,877,854,825,812,769,734,705,667,636,628,615 ;{ }^{11} \mathrm{~B} \mathrm{NMR}$ $\left(129 \mathrm{MHz}, \mathrm{CDCl}_{3}\right) \delta 30.6$; HRMS (ESI-) $m / z[\mathrm{M}-\mathrm{H}]^{-}$calcd for $\mathrm{C}_{19} \mathrm{H}_{20} \mathrm{BO}_{2}$ 291.1560; observed 291.1571. 
fluorene-2-Bpin was identified in comparison with the reported data9 .

fluorene-3-Bpin: ${ }^{1} \mathrm{H}$ NMR $\left(500 \mathrm{MHz}, \mathrm{CDCl}_{3}\right) \delta 1.38(\mathrm{~s}, 12 \mathrm{H}, a), 3.89(\mathrm{~s}, 2 \mathrm{H}, d), 7.23-7.38(\mathrm{~m}, 2 \mathrm{H}$, $f, g), 7.50-7.57(\mathrm{~m}, 2 \mathrm{H}, c, e), 7.76-7.85(\mathrm{~m}, 2 \mathrm{H}, b, h), 8.24(\mathrm{~s}, 1 \mathrm{H}, i) ;{ }^{13} \mathrm{C} \mathrm{NMR}\left(126 \mathrm{MHz}, \mathrm{CDCl}_{3}\right)$ $\delta 24.9$ (4C), 37.1 (1C), 83.8 (2C), 120.1 (1C), 124.4 (1C), 124.9 (1C), 126.2 (1C), 126.6 (1C), 126.7 (1C), 133.3 (1C), 141.2 (1C), 141.6 (1C), 142.9 (1C), 146.6 (1C). The NMR signal of the carbon $\alpha$ to the boron was not observed.

Synthesis of 4,4,5,5-tetramethyl-2-(2-triphenylenyl)-1,3,2-dioxaborolane (2h: triphenylene-2Bpin)

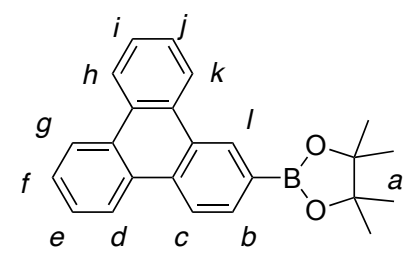

To a Schlenk tube were added boron triiodide $(78.1 \mathrm{mg}, 0.20 \mathrm{mmol})$, triphenylene $(0.180 \mathrm{~g}, 0.79$ $\mathrm{mmol})$ and 1,2,4-trichlorobenzene $(1.6 \mathrm{~mL})$ at room temperature under a nitrogen atmosphere. After stirring at $160{ }^{\circ} \mathrm{C}$ for $8 \mathrm{~h}$, the reaction mixture was allowed to cool to room temperature and then hydrogen iodide was removed in vacuo. Triethylamine $(0.340 \mathrm{~mL}, 2.4 \mathrm{mmol})$ and pinacol $(71.4 \mathrm{mg}$, $0.60 \mathrm{mmol}$ ) were added to the reaction mixture at room temperature. After stirring at room temperature for $0.5 \mathrm{~h}$, the solvent was removed in vacuo $\left(100^{\circ} \mathrm{C}, 0.23 \mathrm{mmHg}\right)$. The yield of the title compound in the crude product was determined to be $83 \%$ by ${ }^{1} \mathrm{H}$ NMR using $1,1,2,2$ tetrachloroethane as an internal standard. The crude product was purified by GPC (eluent: toluene) to obtain the title compound (47.3 $\mathrm{mg}, 67 \%$ yield, $>98 \%$ pure on ${ }^{1} \mathrm{H}$ NMR analysis) as a white solid. IR (neat): $\mathrm{cm}^{-1} 3088$ (Ar-H), 2976, 2931, 1926, 1612, 1583, 1541, 1506, 1489, 1469, 1398, 1386, 1363, 1328, 1311, 1292, 1271, 1213, 1165, 1141, 1120, 1097, 1051, 1026, 960, 947, 904, 867, 844, 823, 781, 750, 723, 702, 694, 678, 634, 624, 615; mp: 184.0-185.2 ${ }^{\circ} \mathrm{C} ;{ }^{1} \mathrm{H} \mathrm{NMR}\left(400 \mathrm{MHz}, \mathrm{CDCl}_{3}\right)$ $\delta 1.43(\mathrm{~s}, 12 \mathrm{H}, a), 7.63-7.69(\mathrm{~m}, 4 \mathrm{H}, e, f, i, j), 8.06(\mathrm{~d}, J=8.2 \mathrm{~Hz}, 1 \mathrm{H}, b), 8.64-8.71(\mathrm{~m}, 4 \mathrm{H}, d, g, h$, k), 8.81-8.83 (m, 1H, c), $\left.9.14(\mathrm{~s}, 1 \mathrm{H}, l) ;{ }^{13} \mathrm{C} \mathrm{NMR} \mathrm{(101} \mathrm{MHz,} \mathrm{CDCl}_{3}\right) \delta 25.0(4 \mathrm{C}), 84.0(2 \mathrm{C}), 122.4$ (1C), 123.1 (1C), 123.3 (1C), 123.7 (2C), 127.1 (2C), 127.2 (1C), 127.6 (1C), 129.0 (1C), 129.6 (1C), 129.7 (1C), 129.9 (1C), 130.3 (1C), 130.5 (1C), 132.0 (1C), 132.8 (1C). The NMR signal of the carbon $\alpha$ to the boron was not observed.; ${ }^{11} \mathrm{~B}$ NMR $\left(129 \mathrm{MHz}, \mathrm{CDCl}_{3}\right) \delta 30.1$; HRMS (ESI+) $m / z$ $[\mathrm{M}+\mathrm{Na}]^{+}$calcd for $\mathrm{C}_{24} \mathrm{H}_{23} \mathrm{BO}_{2} \mathrm{Na} 377.1693$; observed 377.1680 .

(8) Tang, M. L.; Roberts, M. E.; Locklin, J. J.; Ling, M. M.; Meng, H.; Bao, Z. Chem. Mater. 2006, 18,6250 . 


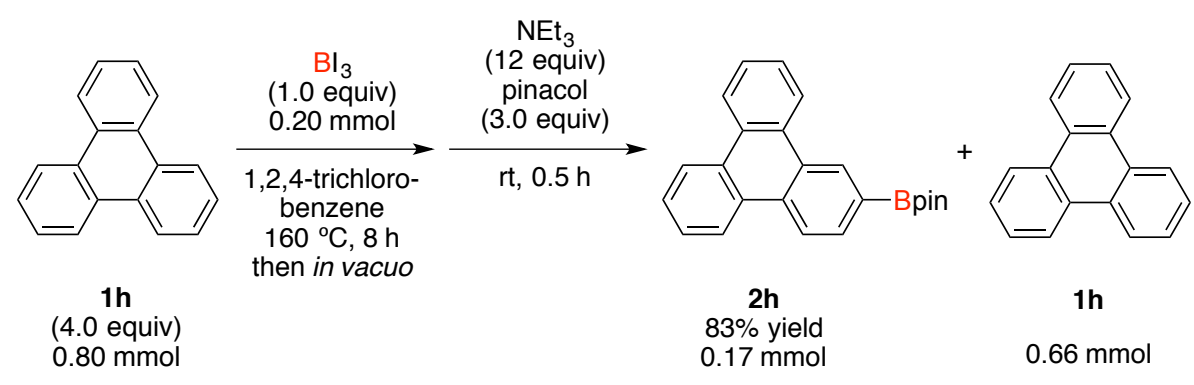

Synthesis of 4,4,5,5-tetramethyl-2-(pyren-2-yl)-1,3,2-dioxaborolane (2i: pyrene-2-Bpin)

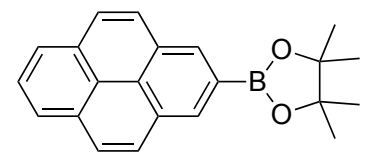

To a Schlenk tube were boron triiodide $(78.3 \mathrm{mg}, 0.20 \mathrm{mmol})$, pyrene $(81.0 \mathrm{mg}, 0.40 \mathrm{mmol})$ and 1,2,4-trichlorobenzene $(2.0 \mathrm{~mL})$ at room temperature under a nitrogen atmosphere. After stirring at $180{ }^{\circ} \mathrm{C}$ for $8 \mathrm{~h}$, the reaction mixture was allowed to cool to room temperature and then hydrogen iodide was removed in vacuo. Triethylamine $(0.335 \mathrm{~mL}, 2.4 \mathrm{mmol})$ and pinacol $(71.1 \mathrm{mg}, 0.60 \mathrm{mmol})$ were added to the reaction mixture at room temperature. After stirring at room temperature for $0.5 \mathrm{~h}$, the solvent was removed in vacuo $\left(100^{\circ} \mathrm{C}, 0.23 \mathrm{mmHg}\right)$. The yield of the title compound in the crude product was determined to be $56 \%$ by ${ }^{1} \mathrm{H}$ NMR using 1,1,2,2-tetrachloroethane as an internal standard. The crude product was purified by GPC (eluent: toluene) to obtain the title compound (26.9 $\mathrm{mg}, 41 \%$ yield, $>97 \%$ pure on ${ }^{1} \mathrm{H}$ NMR analysis) as a yellow solid. The compound was identified in comparison with the reported data ${ }^{10}$.

\section{Synthesis of 4,4,5,5-tetramethyl-2-(perylen-2-yl)-1,3,2-dioxaborolane ( $2 \mathrm{j}$ : perylene-2-Bpin)}

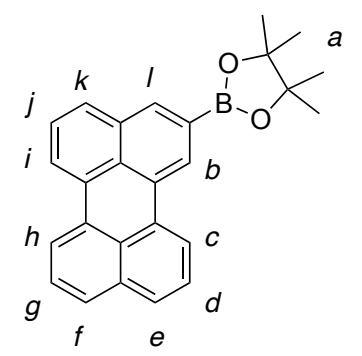

To a Schlenk tube were boron triiodide $(78.1 \mathrm{mg}, 0.20 \mathrm{mmol})$, perylene $(0.101 \mathrm{~g}, 0.40 \mathrm{mmol})$ and 1,2,4-trichlorobenzene $(2.0 \mathrm{~mL})$ at room temperature under a nitrogen atmosphere. After stirring at $200{ }^{\circ} \mathrm{C}$ for $8 \mathrm{~h}$, the reaction mixture was allowed to cool to room temperature and then hydrogen iodide was removed in vacuo. Triethylamine $(0.335 \mathrm{~mL}, 2.4 \mathrm{mmol})$ and pinacol $(70.9 \mathrm{mg}, 0.60 \mathrm{mmol})$

(9) Oniwa, K.; Kikuchi, H.; Shimotani, H.; Ikeda, S.; Asao, N.; Yamamoto, Y.; Tanigaki, K.; Jin, T. Chem. Commun. 2016, 52, 4800. 
were added to the reaction mixture at room temperature. After stirring at room temperature for $0.5 \mathrm{~h}$, the solvent was removed in vacuo $\left(100^{\circ} \mathrm{C}, 0.23 \mathrm{mmHg}\right)$. The yield of the title compound in the crude product was determined to be $37 \%$ by ${ }^{1} \mathrm{H}$ NMR using 1,1,2,2-tetrachloroethane as an internal standard. The crude product was purified by GPC (eluent: toluene) to obtain the title compound (22.1 mg, 29\% yield, $>97 \%$ pure on ${ }^{1} \mathrm{H}$ NMR analysis) as a yellow solid. IR (neat): $\mathrm{cm}^{-1} 3055$ (Ar-H), 2968, 2928, $1622,1593,1512,1462,1418,1371,1315,1275,1217,1206,1169,1142,1057,974,895,883,853$, 837, 802, 768, 733, 712, 694, 671, 648; mp: 234.5-235.0 ${ }^{\circ} \mathrm{C} ;{ }^{1} \mathrm{H}$ NMR (400 MHz, $\left.\mathrm{CDCl}_{3}\right) \delta 1.42(\mathrm{~s}$, 12H, a), 7.44-7.49 (m, 3H, $d, g, j), 7.67(\mathrm{~d}, J=8.2 \mathrm{~Hz}, 2 \mathrm{H}, e, f), 7.71(\mathrm{~d}, J=8.2 \mathrm{~Hz}, 1 \mathrm{H}, k), 8.17(\mathrm{~d}$, $J=7.3 \mathrm{~Hz}, 1 \mathrm{H}, h$ or $i), 8.18(\mathrm{~s}, 1 \mathrm{H}, l), 8.21(\mathrm{~d}, J=7.3 \mathrm{~Hz}, 1 \mathrm{H}, h$ or $i), 8.33(\mathrm{~d}, J=7.8 \mathrm{~Hz}, 1 \mathrm{H}, c)$, 8.55 (s, 1H, b); ${ }^{13} \mathrm{C}$ NMR (126 MHz, $\left.\mathrm{CDCl}_{3}\right) \delta 24.9$ (4C), 84.1 (2C), 120.0 (1C), 120.6 (1C), 121.1 (1C), 125.0 (1C), 126.4 (1C), 126.5 (1C), 126.5 (1C), 127.7 (1C), 127.8 (1C), 128.5 (1C), 128.8 (1C), 130.3 (1C), 130.3 (1C), 131.2 (1C), 131.2 (1C), 131.3 (1C), 134.0 (1C), 134.7 (1C), 135.9 (1C). The NMR signal of the carbon $\alpha$ to the boron was not observed.; ${ }^{11} \mathrm{~B}$ NMR $\left(129 \mathrm{MHz}, \mathrm{CDCl}_{3}\right) \delta 30.5$; HRMS (ESI+) $m / z[\mathrm{M}+\mathrm{Na}]^{+}$calcd for $\mathrm{C}_{26} \mathrm{H}_{23} \mathrm{BO}_{2} \mathrm{Na} 401.1693$; observed 401.1688.

\section{Synthesis of 4,4,5,5-tetramethyl-2-(3-phenoxyphenyl)-1,3,2-dioxaborolane and 4,4,5,5- tetramethyl-2-(4-phenoxyphenyl)-1,3,2-dioxaborolane (2k:diphenylether-3-Bpin and diphenylether-4-Bpin)}

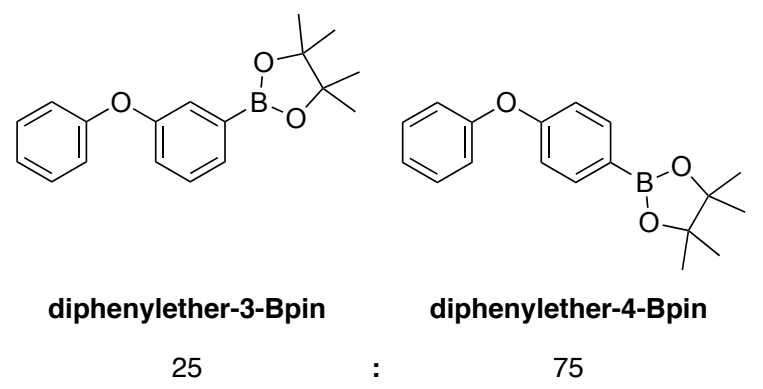

To a Schlenk tube were added boron triiodide $(0.156 \mathrm{~g}, 0.40 \mathrm{mmol})$, diphenylether $(0.287 \mathrm{~g}, 1.6$ $\mathrm{mmol})$ and 1,2,4-trichlorobenzene $(1.6 \mathrm{~mL})$ at room temperature under a nitrogen atmosphere. After stirring at $180{ }^{\circ} \mathrm{C}$ for $12 \mathrm{~h}$, the reaction mixture was allowed to cool to room temperature and then hydrogen iodide was removed in vacuo. Triethylamine $(0.670 \mathrm{~mL}, 4.8 \mathrm{mmol})$ and pinacol $(0.142 \mathrm{~g}$, $1.2 \mathrm{mmol}$ ) were added to the reaction mixture at room temperature. After stirring at room temperature for $0.5 \mathrm{~h}$, the solvent was removed in vacuo $\left(100^{\circ} \mathrm{C}, 0.23 \mathrm{mmHg}\right)$. The yields of the title compounds in the crude product were determined to be $15 \%$ and $64 \%$ (total yield: $79 \%$ ) by ${ }^{1} \mathrm{H} \mathrm{NMR}$ using $1,1,2,2$ tetrachloroethane as an internal standard. The crude product was purified by GPC (eluent: toluene) to obtain a 25:75 mixture of diphenylether-3-Bpin and diphenylether-4-Bpin $(60.1 \mathrm{mg}, 51 \%$ yield, $>98 \%$ pure on ${ }^{1} \mathrm{H}$ NMR analysis) as a yellow liquid. The compounds were identified in comparison 
with the reported data ${ }^{11}$.

Synthesis of 9-(phenyl)-2-(4,4,5,5-tetramethyl-1,3,2-dioxaborolane-2-yl)-9H-carbazole and 9(phenyl)-3-(4,4,5,5-tetramethyl-1,3,2-dioxaborolane-2-yl)- 9H-carbazole (2l: 9phenylcarbazole-2-Bpin and 9-phenylcarbazole-3-Bpin)

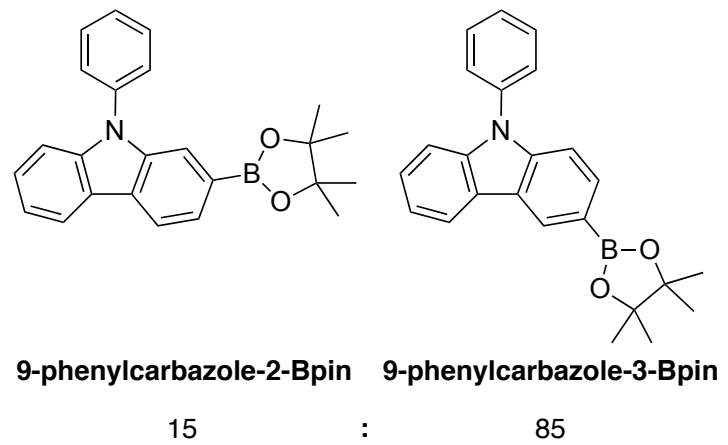

To a Schlenk tube were added boron triiodide $(39.2 \mathrm{mg}, 0.10 \mathrm{mmol})$, 9-phenylcarbazole $(98.4 \mathrm{mg}$, $0.40 \mathrm{mmol})$ and 1,2,4-trichlorobenzene $(1.0 \mathrm{~mL})$ at room temperature under a nitrogen atmosphere. After stirring at $180{ }^{\circ} \mathrm{C}$ for $6 \mathrm{~h}$, the reaction mixture was allowed to cool to room temperature and then hydrogen iodide was removed in vacuo. Triethylamine $(0.170 \mathrm{~mL}, 1.2 \mathrm{mmol})$ and pinacol $(35.1$ $\mathrm{mg}, 0.30 \mathrm{mmol}$ ) were added to the reaction mixture at room temperature. After stirring at room temperature for $0.5 \mathrm{~h}$, the solvent was removed in vacuo $\left(100^{\circ} \mathrm{C}, 0.23 \mathrm{mmHg}\right)$. The yields of the title compounds in the crude product were determined to be $11 \%$ and $60 \%$ (total yield: $71 \%$ ) by ${ }^{1} \mathrm{H}$ NMR using 1,1,2,2-tetrachloroethane as an internal standard. The crude product was purified by GPC (eluent: toluene) to obtain a 15:85 mixture of 9-phenylcarbazole-2-Bpin and 9-phenylcarbazole-3Bpin (19.2 mg, 52\% yield, $>98 \%$ pure on ${ }^{1} \mathrm{H}$ NMR analysis) as a white solid. The compounds were identified in comparison with the reported data ${ }^{12}$.

Synthesis of $(4,4,5,5$-tetramethyl-1,3,2-dioxaborolane-2-yl)ferrocene ( $2 \mathrm{~m}$ : ferrocene-Bpin)

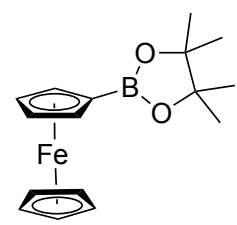

To a Schlenk tube were added boron triiodide $(0.157 \mathrm{~g}, 0.40 \mathrm{mmol})$, ferrocene $(0.298 \mathrm{~g}, 1.6 \mathrm{mmol})$ and $o$-dichlorobenzene $(1.6 \mathrm{~mL})$ at $0{ }^{\circ} \mathrm{C}$ under a nitrogen atmosphere. After stirring at $0{ }^{\circ} \mathrm{C}$ for $16 \mathrm{~h}$, the reaction mixture was allowed to warm to room temperature and then hydrogen iodide was

(10) Bheeter, B. C.; Chowdhury, D. A.; Adam, R.; Jackstell, R.; Beller, M. Org. Biomol. Chem. 2015, 13, 10336.

(11) (a) Kim, S. H.; Cho, I.; Sim, M. K.; Park, S.; Park, S. Y. J. Mater. Chem. 2011, 21, 9139.

(b) Keerthi, A.; Valiyaveettil, S. J. Phys. Chem. B 2012, 116, 4603. 
removed in vacuo. Triethylamine $(0.670 \mathrm{~mL}, 4.8 \mathrm{mmol})$ and pinacol $(0.142 \mathrm{~g}, 1.2 \mathrm{mmol})$ were added to the reaction mixture at room temperature. After stirring at room temperature for $0.5 \mathrm{~h}$, the solvent was removed in vacuo $\left(100{ }^{\circ} \mathrm{C}, 0.23 \mathrm{mmHg}\right)$. The yield of the title compound in the crude product was determined to be $70 \%$ by ${ }^{1} \mathrm{H}$ NMR using 1,1,2,2-tetrachloroethane as an internal standard. The crude product was purified by GPC (eluent: toluene) to obtain the title compound $(77.4 \mathrm{mg}, 62 \%$ yield, $>99 \%$ pure on ${ }^{1} \mathrm{H}$ NMR analysis) as an orange solid. The compound was identified in comparison with the reported data ${ }^{13}$.

\section{Synthesis of (5,6,7,8-tetrahydronaphthalen-2-yl)boronic acid (3: tetralin-6-B(OH) $)$}

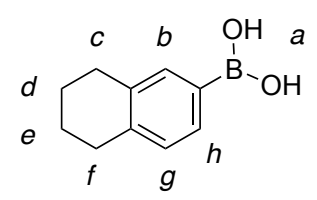

To a Schlenk tube were added boron triiodide $(0.392 \mathrm{~g}, 1.0 \mathrm{mmol})$ and 1,2,3,4-tetrahydronaphthalene $(2.05 \mathrm{~mL}, 15 \mathrm{mmol})$ at room temperature under a nitrogen atmosphere. After stirring at $160^{\circ} \mathrm{C}$ for 16 $\mathrm{h}$, the reaction mixture was allowed to cool to room temperature and then hydrogen iodide was

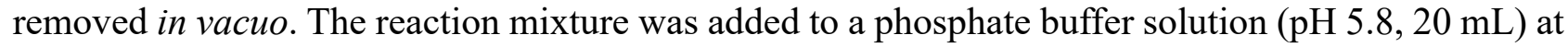
$0{ }^{\circ} \mathrm{C}$ through a cannula. After stirring at room temperature for $0.5 \mathrm{~h}$, the reaction mixture was extracted with toluene $(20 \mathrm{~mL}$, three times). The organic layer was washed with brine $(20 \mathrm{~mL})$ and dehydrated with anhydrous magnesium sulfate and condensed in vacuo. The yield of the title compound in the crude product was determined to be $89 \%$ by ${ }^{1} \mathrm{H}$ NMR using dibromomethane as an internal standard. The crude product was purified by GPC (eluent: 1,2-dichloroethane) to obtain the title compound $\left(0.121 \mathrm{~g}, 69 \%\right.$ yield, $>97 \%$ pure on ${ }^{1} \mathrm{H}$ NMR analysis) as a white solid. IR (neat): $\mathrm{cm}^{-}$ ${ }^{1} 3005,2928,2857$ (Ar-H), 1609, 1564, 1506, 1406, 1360, 1337, 1308, 1280, 1234, 1177, 1128, 1084, $1065,991,961,939,924,858,831,816,735,725,704,691,662 ; \mathrm{mp}: 84.5-86.0{ }^{\circ} \mathrm{C} ;{ }^{1} \mathrm{H}$ NMR $(400$ $\left.\mathrm{MHz},\left(\mathrm{CD}_{3}\right)_{2} \mathrm{SO}\right) \delta 1.72-1.73(\mathrm{~m}, 4 \mathrm{H}, d, e), 2.68-2.72(\mathrm{~m}, 4 \mathrm{H}, c, f), 6.99(\mathrm{~d}, J=8.2 \mathrm{~Hz}, 1 \mathrm{H}, g), 7.47$ $(\mathrm{d}, J=8.2 \mathrm{~Hz}, 1 \mathrm{H}, h), 7.48(\mathrm{~s}, 1 \mathrm{H}, b) 7.84(\mathrm{~s}, 2 \mathrm{H}, a) ;{ }^{13} \mathrm{C} \mathrm{NMR}\left(101 \mathrm{MHz},\left(\mathrm{CD}_{3}\right)_{2} \mathrm{SO}\right) \delta 22.7(1 \mathrm{C})$, 22.9 (1C), 28.8 (1C), 28.9 (1C), 128.0 (1C), 131.2 (1C), 135.1 (1C), 135.3 (1C), 138.6 (1C). The NMR signal of the carbon $\alpha$ to the boron was not observed.; ${ }^{11} \mathrm{~B} \mathrm{NMR}\left(129 \mathrm{MHz},\left(\mathrm{CD}_{3}\right)_{2} \mathrm{SO}\right) \delta 27.6$; HRMS (ESI-) $m / z$ [M-H] $]^{-}$calcd for $\mathrm{C}_{10} \mathrm{H}_{12} \mathrm{BO}_{2}$ 175.0932; observed 175.0929.

\section{Synthesis of potassium trifluoro(5,6,7,8-tetrahydro-2-naphthalenyl)borate (4: tetralin-6- $\left.\mathrm{BF}_{3} \mathbf{K}\right)$}




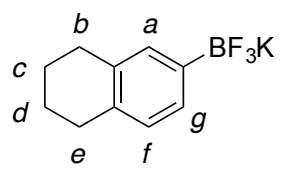

To a Schlenk tube were added boron triiodide (0.392 g, $1.0 \mathrm{mmol})$ and 1,2,3,4-tetrahydronaphthalene $(2.04 \mathrm{~mL}, 15 \mathrm{mmol})$ at room temperature under a nitrogen atmosphere. After stirring at $160^{\circ} \mathrm{C}$ for 16 $\mathrm{h}$, the reaction mixture was allowed to cool to room temperature and then hydrogen iodide was removed in vacuo. The reaction mixture was slowly added to a potassium fluoride $(0.349 \mathrm{~g}, 6.0 \mathrm{mmol})$ in acetonitrile $(10 \mathrm{~mL})$ at $0{ }^{\circ} \mathrm{C}$ through a cannula. After stirring at $40^{\circ} \mathrm{C}$ for $1 \mathrm{~h}$, the reaction mixture was filtered, and the filtrate was condensed in vacuo. The yield of the title compound in the crude product was determined to be $86 \%$ by ${ }^{1} \mathrm{H}$ NMR using dibromomethane as an internal standard. The crude product was washed with hexane to obtain the title compound $(0.190 \mathrm{~g}, 80 \%$ yield, $>97 \%$ pure on ${ }^{1} \mathrm{H}$ NMR analysis) as a white solid. IR (neat): $\mathrm{cm}^{-1}$ 2924, 2854, 2835 (Ar-H), 2358, 1697, 1664, 1606, 1558, 1494, 1435, 1415, 1288, 1255, 1234, 1184, 1153, 1126, 1051, 1006, 974, 914, 825, 812, 771, 740, 723, 704, 684, 667, 626; mp: 98.0-99.0 ${ }^{\circ} \mathrm{C}$; ${ }^{1} \mathrm{H}$ NMR (400 MHz, $\left.\left(\mathrm{CD}_{3}\right)_{2} \mathrm{CO}\right) \delta 1.69-1.70$ $(\mathrm{m}, 4 \mathrm{H}, c, d), 2.60-2.66(\mathrm{~m}, 4 \mathrm{H}, b, e), 6.76(\mathrm{~d}, J=7.2 \mathrm{~Hz}, 1 \mathrm{H}, f), 7.00-7.03(\mathrm{~m}, 2 \mathrm{H}, a, g) ;{ }^{13} \mathrm{C} \mathrm{NMR}$ (126 MHz, $\left.\left(\mathrm{CD}_{3}\right)_{2} \mathrm{CO}\right) \delta 24.4$ (2C), 24.5 (2C), 127.7 (1C), 129.8 (1C), 133.2 (1C), 133.8 (1C), 134.7 (1C). The NMR signal of the carbon $\alpha$ to the boron was not observed.; ${ }^{19} \mathrm{~F}$ NMR $(376 \mathrm{MHz}$, $\left.\left(\mathrm{CD}_{3}\right)_{2} \mathrm{CO}\right) \delta-147.6 ;{ }^{11} \mathrm{~B} \mathrm{NMR}\left(129 \mathrm{MHz},\left(\mathrm{CD}_{3}\right)_{2} \mathrm{CO}\right) \delta 2.94$; HRMS (ESI-) $\mathrm{m} / z\left[\mathrm{M}-\mathrm{K}^{+}\right]^{-}$calcd for $\mathrm{C}_{10} \mathrm{H}_{12} \mathrm{BF}_{3}$ 199.0908; observed 199.0908.

\section{Synthesis of 10-mesityl-2,8-dimethyl-5-(p-tolyl)-10H-dibenzo[b,e $][1,4]$ oxaborine $(5)$}

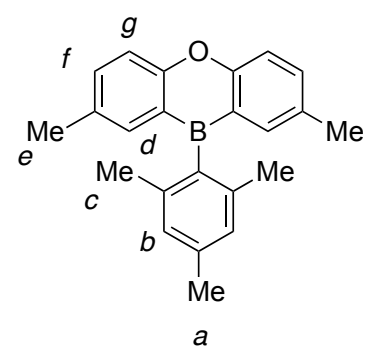

To a Schlenk tube were added boron triiodide $(0.158 \mathrm{~g}, 0.40 \mathrm{mmol})$, di- $p$-tolylether $(41.0 \mathrm{mg}, 0.21$ $\mathrm{mmol})$ and 1,2,4-trichlorobenzene $(2.0 \mathrm{~mL})$ at room temperature under a nitrogen atmosphere. After stirring at $240{ }^{\circ} \mathrm{C}$ for $24 \mathrm{~h}$, the reaction mixture was allowed to cool to room temperature and then hydrogen iodide was removed in vacuo. After addition of 2-mesitylmagnesium bromide in THF (1.00 $\mathrm{mL}, 1.20 \mathrm{M}, 1.2 \mathrm{mmol}$ ), the reaction mixture was stirred at room temperature for $0.5 \mathrm{~h}$. After the reaction mixture was filtered with a pad of Florisil ${ }^{\circledR}$ (eluent: toluene), the solvent was removed in vacuo $\left(100{ }^{\circ} \mathrm{C}, 0.23 \mathrm{mmHg}\right)$. The yield of the title compound in the crude product was determined to be $87 \%$ by ${ }^{1} \mathrm{H}$ NMR using 1,1,2,2-tetrachloroethane as an internal standard. The crude product was purified by GPC (eluent: toluene) to obtain the title compound ( $48.3 \mathrm{mg}, 74 \%$ yield, $>98 \%$ pure on 
${ }^{1} \mathrm{H}$ NMR analysis) as a white solid. IR (neat): $\mathrm{cm}^{-1} 3012$ (Ar-H), 2922, 2854, 1608, 1575, 1556, 1485, 1450, 1413, 1373, 1332, 1298, 1265, 1215, 1159, 1132, 1124, 1097, 1033, 958, 923, 898, 873, 844, 825, 812, 759, 719, 677, 653, 615; mp: $>300{ }^{\circ} \mathrm{C} ;{ }^{1} \mathrm{H}$ NMR (500 MHz, $\left.\mathrm{CDCl}_{3}\right) \delta 1.99(\mathrm{~s}, 6 \mathrm{H}, c), 2.35$ $(\mathrm{s}, 6 \mathrm{H}, e), 2.41(\mathrm{~s}, 3 \mathrm{H}, a), 6.96(\mathrm{~s}, 2 \mathrm{H}, b), 7.48(\mathrm{~d}, J=2.3 \mathrm{~Hz}, 2 \mathrm{H}, f), 7.49-7.55(\mathrm{~m}, 4 \mathrm{H}, d, g) ;{ }^{13} \mathrm{C}$ NMR (126 MHz, $\mathrm{CDCl}_{3}$ ) $\delta 20.7$ (2C), 21.3 (1C), 22.9 (2C), 117.4 (2C), $126.9(2 \mathrm{C}), 131.5(2 \mathrm{C}), 135.5$ (2C), 135.9 (2C), 136.8 (2C), 138.9 (2C), 157.7 (2C). The NMR signal of the carbon $\alpha$ to the boron was not observed.; ${ }^{11} \mathrm{~B}$ NMR (129 $\left.\mathrm{MHz} \mathrm{CDCl}_{3}\right) \delta 55.4$; HRMS (DART) $m / z[\mathrm{M}+\mathrm{H}]^{+}$calcd for $\mathrm{C}_{23} \mathrm{H}_{24} \mathrm{BO} 327.1924$; observed 327.1946.

\section{Synthesis of 10-mesityl-2,8-dimethyl-5-(p-tolyl)-5,10-dihydrodibenzo[b,e][1,4]azaborine (6)}

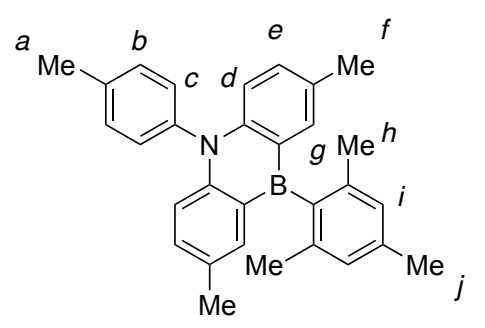

To a Schlenk tube were added boron triiodide $(0.118 \mathrm{~g}, 0.30 \mathrm{mmol})$, tri-p-tolylamine $(57.6 \mathrm{mg}, 0.20$ mmol) and $o$-dichlorobenzene $(2.0 \mathrm{~mL})$ at room temperature under a nitrogen atmosphere. After stirring at $150{ }^{\circ} \mathrm{C}$ for $2 \mathrm{~h}$, the reaction mixture was allowed to cool to room temperature and then hydrogen iodide was removed in vacuo. After addition of 2-mesitylmagnesium bromide in THF $(0.750 \mathrm{~mL}, 1.20 \mathrm{M}, 0.90 \mathrm{mmol})$, the reaction mixture was stirred at room temperature for $0.5 \mathrm{~h}$. After the reaction mixture was filtered with a pad of Florisil ${ }^{\circledR}$ (eluent: toluene), the solvent was removed in vacuo $\left(100{ }^{\circ} \mathrm{C}, 0.23 \mathrm{mmHg}\right)$. The yield of the title compound in the crude product was determined to be $90 \%$ by ${ }^{1} \mathrm{H}$ NMR using 1,1,2,2-tetrachloroethane as an internal standard. The crude product was purified by GPC (eluent: toluene) to obtain the title compound $(68.2 \mathrm{mg}, 82 \%$ yield, $>98 \%$ pure on ${ }^{1} \mathrm{H}$ NMR analysis) as a yellow solid. IR (neat): $\mathrm{cm}^{-1}$ 2914, 2854 (Ar-H), 1608, 1562, 1544, 1510, $1489,1454,1423,1377,1323,1238,1163,1095,1039,1020,958,941,891,848,815,808,725,678$, 655, 621; mp: $>300{ }^{\circ} \mathrm{C} ;{ }^{1} \mathrm{H}$ NMR (500 MHz, $\left.\mathrm{CDCl}_{3}\right) \delta 2.04(\mathrm{~s}, 6 \mathrm{H}, h), 2.32(\mathrm{~s}, 6 \mathrm{H}, f), 2.44(\mathrm{~s}, 3 \mathrm{H}, j)$, $2.55(\mathrm{~s}, 3 \mathrm{H}, a), 6.78(\mathrm{~d}, J=8.7 \mathrm{~Hz}, 2 \mathrm{H}, d), 6.99(\mathrm{~s}, 2 \mathrm{H}, i), 7.25(\mathrm{~d}, J=7.8 \mathrm{~Hz}, 2 \mathrm{H}, c), 7.30(\mathrm{dd}, J=$ 2.3, 8.7 Hz 2H,e), $7.48(\mathrm{~d}, J=7.8 \mathrm{~Hz}, 2 \mathrm{H}, b), 7.61(\mathrm{~s}, 2 \mathrm{H}, g) ;{ }^{13} \mathrm{C} \mathrm{NMR}\left(126 \mathrm{MHz}, \mathrm{CDCl}_{3}\right) \delta 20.6$ (2C), 21.4 (2C), 23.3 (2C), 116.9 (2C), 126.7 (2C), 128.4 (1C), 130.0 (2C), 131.4 (2C), 134.1 (2C), 135.9 (2C), 136.1 (1C), 138.6 (1C), 139.2 (2C), 139.4 (1C), 144.9 (2C). The NMR signal of the carbon $\alpha$ to the boron was not observed.; ${ }^{11} \mathrm{~B}$ NMR $\left(129 \mathrm{MHz}, \mathrm{CDCl}_{3}\right) \delta 50.9$; HRMS (DART) $m / z$ $[\mathrm{M}+\mathrm{H}]^{+}$calcd for $\mathrm{C}_{30} \mathrm{H}_{31} \mathrm{BN} 416.2555$; observed 416.2541.

Synthesis of 2,2'-(2,7-pyrenediyl)bis[4,4,5,5-tetramethyl-1,3,2-dioxaborolane] (7: pyrene-2,7(Bpin)2) 


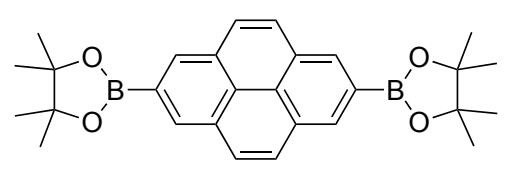

To a Schlenk tube were boron triiodide $(0.313 \mathrm{~g}, 0.80 \mathrm{mmol})$, pyrene $(40.5 \mathrm{mg}, 0.20 \mathrm{mmol})$ and 1,2,4trichlorobenzene $(2.0 \mathrm{~mL})$ at room temperature under a nitrogen atmosphere. After stirring at $240{ }^{\circ} \mathrm{C}$ for $16 \mathrm{~h}$, the reaction mixture was allowed to cool to room temperature and then hydrogen iodide was removed in vacuo. Triethylamine $(1.33 \mathrm{~mL}, 9.6 \mathrm{mmol})$ and pinacol $(0.284 \mathrm{~g}, 2.4 \mathrm{mmol})$ were added to the reaction mixture at room temperature. After stirring at room temperature for $0.5 \mathrm{~h}$, the solvent was removed in vacuo $\left(100^{\circ} \mathrm{C}, 0.23 \mathrm{mmHg}\right)$. The yield of the title compound in the crude product was determined to be $51 \%$ by ${ }^{1} \mathrm{H}$ NMR using 1,1,2,2-tetrachloroethane as an internal standard. The crude product was purified by GPC (eluent: 1,2-dichloroethane) to obtain the title compound (35.4 mg, 39\% yield, $>98 \%$ pure on ${ }^{1} \mathrm{H}$ NMR analysis) as a white solid. The compound was identified in comparison with the reported data ${ }^{14}$.

\section{Synthesis of 2,2',2',2'"'-(2,5,8,11-perlyenetetrayl)tetrakis[4,4,5,5-tetramethyl-1,3,2 - dioxaborolane] (8: perylene-2,5,8,11-(Bpin) 4$)$}

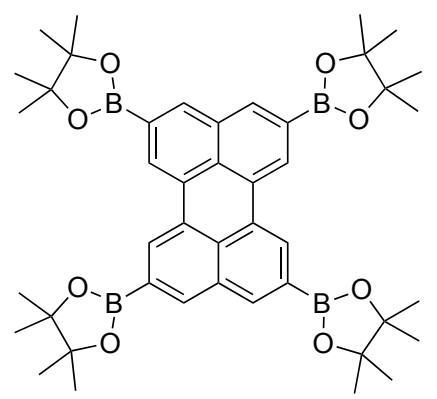

To a Schlenk tube were boron triiodide $(0.626 \mathrm{~g}, 1.6 \mathrm{mmol})$, perylene $(50.5 \mathrm{mg}, 0.20 \mathrm{mmol})$ and 1,2,4-trichlorobenzene $(2.0 \mathrm{~mL})$ at room temperature under a nitrogen atmosphere. After stirring at $240{ }^{\circ} \mathrm{C}$ for $16 \mathrm{~h}$, the reaction mixture was allowed to cool to room temperature and then hydrogen iodide was removed in vacuo. Triethylamine $(2.68 \mathrm{~mL}, 19 \mathrm{mmol})$ and pinacol $(0.567 \mathrm{~g}, 4.8 \mathrm{mmol})$ were added to the reaction mixture at room temperature. After stirring at room temperature for $0.5 \mathrm{~h}$, the solvent was removed in vacuo $\left(100^{\circ} \mathrm{C}, 0.23 \mathrm{mmHg}\right)$. The yield of the title compound in the crude product was determined to be $36 \%$ by ${ }^{1} \mathrm{H}$ NMR using 1,1,2,2-tetrachloroethane as an internal standard. The crude product was purified by GPC (eluent: 1,2-dichloroethane) to obtain the title compound (49.5 mg, 33\% yield, $>98 \%$ pure on ${ }^{1} \mathrm{H}$ NMR analysis) as a yellow solid. The compound was identified in comparison with the reported data ${ }^{13}$

(13) Coventry, N. D.; Batsanov, S. A.; Goeta, E. A.; Howard, K. A. J.; Marder, B. T.; Perutz, R. N.; Chem. Commun. 2005, 2172. 


\section{Synthesis of 1,1',3,3'-tetra(4,4,5,5-tetramethyl-1,3,2-dioxaborolane-2-yl)feroccene (9)}

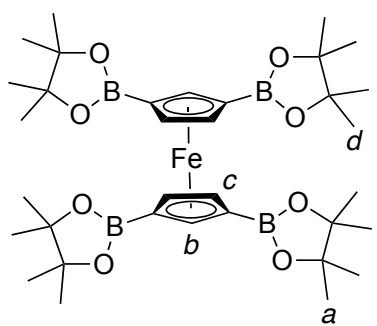

To a Schlenk tube were added boron triiodide $(0.940 \mathrm{~g}, 2.4 \mathrm{mmol})$, ferrocene $(37.2 \mathrm{mg}, 0.20 \mathrm{mmol})$ and 1,2,4-trichlorobenzene $(2.0 \mathrm{~mL})$ at room temperature under a nitrogen atmosphere. After stirring at $150^{\circ} \mathrm{C}$ for $16 \mathrm{~h}$, the reaction mixture was allowed to cool to room temperature and then hydrogen iodide was removed in vacuo. Triethylamine $(4.00 \mathrm{~mL}, 29 \mathrm{mmol})$ and pinacol $(0.851 \mathrm{~g}, 7.2 \mathrm{mmol})$ were added to the reaction mixture at $0{ }^{\circ} \mathrm{C}$. After stirring at room temperature for $0.5 \mathrm{~h}$, the solvent was removed in vacuo $\left(100{ }^{\circ} \mathrm{C}, 0.23 \mathrm{mmHg}\right)$. After washing with hexane, the yield of the title compound in the crude product was determined to be $39 \%$ by ${ }^{1} \mathrm{H}$ NMR using 1,1,2,2tetrachloroethane as an internal standard. The crude product was purified by GPC (eluent: 1,2dichloroethane) to obtain the title compound ( $42.8 \mathrm{mg}, 31 \%$ yield, $97 \%$ pure on NMR analysis) as a brown solid. IR (neat): $\mathrm{cm}^{-1}$ 2978, 2928, 1491, 1339, 1325, 1310, 1269, 1248, 1215, 1140, 1055, 962, 910, 854, 833, 802, 698; mp: 205.1-205.5 ${ }^{\circ} \mathrm{C},{ }^{1} \mathrm{H}$ NMR $\left(\mathrm{CDCl}_{3}, 400 \mathrm{MHz}\right) \delta 1.34-1.35$ (m, 48H, $a$, d), $4.53(\mathrm{~d}, J=0.80 \mathrm{~Hz}, 4 \mathrm{H}, c), 4.59(\mathrm{~s}, 2 \mathrm{H}, b) ;{ }^{13} \mathrm{C} \mathrm{NMR}\left(\mathrm{CDCl}_{3}, 101 \mathrm{MHz}\right) \delta 25.0(16 \mathrm{C}), 77.3(4 \mathrm{C})$, 81.1 (2C), 83.1 (8C). The NMR signal of the carbon $\alpha$ to the boron was not observed.; ${ }^{11} \mathrm{~B}$ NMR (128 $\mathrm{MHz}, \mathrm{CDCl}_{3}$ ) $\delta$ 31.7; HRMS (MALDI) $m / z[\mathrm{M}]^{+}$calcd for $\mathrm{C}_{34} \mathrm{H}_{54} \mathrm{~B}_{4} \mathrm{FeO}_{8}$ 690.3558, observed 690.3556.

Synthesis of $\quad 2,2^{\prime}$-(5,5'-dimethyl-[1,1'-biphenyl]-3,3'-diyl)(4,4,5,5-tetramethyl-1,3,2dioxaborolane) (10: 3,3'-dimethylbiphenyl-5,5'-(Bpin $\left.)_{2}\right)$

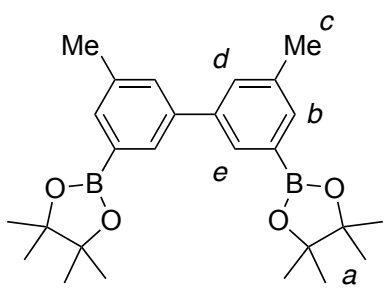

To a Schlenk tube were boron triiodide $(0.783 \mathrm{~g}, 2.0 \mathrm{mmol}), 3$,3'-dimethylbiphenyl $(75.0 \mu \mathrm{L}, 0.41$ $\mathrm{mmol})$ and 1,2,4-trichlorobenzene $(2.0 \mathrm{~mL})$ at room temperature under a nitrogen atmosphere. After stirring at $220{ }^{\circ} \mathrm{C}$ for $16 \mathrm{~h}$, the reaction mixture was allowed to cool to room temperature and then hydrogen iodide was removed in vacuo. Triethylamine $(3.35 \mathrm{~mL}, 24 \mathrm{mmol})$ and pinacol $(0.708 \mathrm{~g}, 6.0$ $\mathrm{mmol}$ ) were added to the reaction mixture at room temperature. After stirring at room temperature for $0.5 \mathrm{~h}$, the solvent was removed in vacuo $\left(100^{\circ} \mathrm{C}, 0.23 \mathrm{mmHg}\right)$. The yield of the title compound in the crude product was determined to be $50 \%$ by ${ }^{1} \mathrm{H}$ NMR using 1,1,2,2-tetrachloroethane as an 
internal standard. The crude product was purified by GPC (eluent: toluene) to obtain the title compound (55.7 mg, 32\% yield, $>98 \%$ pure on ${ }^{1} \mathrm{H}$ NMR analysis) as a yellow solid. IR (neat): $\mathrm{cm}^{-1}$ 2983, 2920 (Ar-H), 1589, 1469, 1398, 1371, 1344, 1307, 1269, 1249, 1209, 1166, 1143, 1114, 1093 , 966, 904, 881, 869, 852, 829, 779, 711, 692, 675, 653; mp: 170.5-171.0 ${ }^{\circ} \mathrm{C} ;{ }^{1} \mathrm{H}$ NMR (400 MHz, $\left.\mathrm{CDCl}_{3}\right) \delta 1.36(\mathrm{~s}, 24 \mathrm{H}, a), 2.41(\mathrm{~s}, 6 \mathrm{H}, c), 7.53(\mathrm{~s}, 2 \mathrm{H}, d) 7.61(\mathrm{~s}, 2 \mathrm{H}, b) 7.84(\mathrm{~s}, 2 \mathrm{H}, e)$; ${ }^{13} \mathrm{C} \mathrm{NMR}$ (101 MHz, $\mathrm{CDCl}_{3}$ ) $\delta 21.3$ (2C), 24.8 (8C), 83.8 (4C), 130.6 (2C), 131.1 (2C), 134.2 (2C), $137.4(2 \mathrm{C})$, 140.6 (2C). The NMR signal of the carbon $\alpha$ to the boron was not observed.; ${ }^{11} \mathrm{~B}$ NMR (129 MHz, $\left.\mathrm{CDCl}_{3}\right) \delta 30.8$; HRMS (ESI+) $\mathrm{m} / z[\mathrm{M}+\mathrm{Na}]^{+}$calcd for $\mathrm{C}_{26} \mathrm{H}_{36} \mathrm{~B}_{2} \mathrm{O}_{4} \mathrm{Na} 457.2706$; observed 457.2711. 


\section{Cartesian coordinates}

1,2,3,4-tetrahydronaphthalene (1a) $\left(C_{2 \mathrm{v}}\right.$ symmetry)

$\mathrm{E}(\mathrm{B} 3 \mathrm{LYP} / 6-31 \mathrm{G}(\mathrm{d}))=-388.28788166$ hartree

\begin{tabular}{|c|c|c|c|c|c|}
\hline \multirow{2}{*}{$\begin{array}{l}\text { Center } \\
\text { Number }\end{array}$} & \multirow{2}{*}{$\begin{array}{l}\text { Atomic } \\
\text { Number }\end{array}$} & \multirow{2}{*}{$\begin{array}{l}\text { Atomic } \\
\text { Type }\end{array}$} & \multicolumn{3}{|c|}{ Coordinates (Angstroms) } \\
\hline & & & $\mathrm{X}$ & $\mathrm{Y}$ & $\mathrm{Z}$ \\
\hline 1 & 6 & 0 & 0.000000 & 0.699593 & 2.597306 \\
\hline 2 & 6 & 0 & 0.000000 & 1.380512 & 1.386697 \\
\hline 3 & 6 & 0 & 0.000000 & 0.698791 & 0.156381 \\
\hline 4 & 6 & 0 & 0.000000 & -0.698791 & 0.156381 \\
\hline 5 & 6 & 0 & 0.000000 & -1.380512 & 1.386697 \\
\hline 6 & 6 & 0 & 0.000000 & -0.699593 & 2.597306 \\
\hline 7 & 1 & 0 & 0.000000 & 1.250648 & 3.534246 \\
\hline 8 & 1 & 0 & 0.000000 & 2.469369 & 1.381738 \\
\hline 9 & 1 & 0 & 0.000000 & -2.469369 & 1.381738 \\
\hline 10 & 1 & 0 & 0.000000 & -1.250648 & 3.534246 \\
\hline 11 & 6 & 0 & 0.000000 & -0.779483 & -2.472416 \\
\hline 12 & 1 & 0 & -0.869339 & -1.118452 & -3.045246 \\
\hline 13 & 1 & 0 & 0.869339 & -1.118452 & -3.045246 \\
\hline 14 & 6 & 0 & 0.000000 & 0.779483 & -2.472416 \\
\hline 15 & 1 & 0 & -0.869339 & 1.118452 & -3.045246 \\
\hline 16 & 1 & 0 & 0.869339 & 1.118452 & -3.045246 \\
\hline 17 & 6 & 0 & 0.000000 & 1.525746 & -1.113834 \\
\hline 18 & 1 & 0 & -0.869809 & 2.194872 & -1.075147 \\
\hline 19 & 1 & 0 & 0.869809 & 2.194872 & -1.075147 \\
\hline 20 & 6 & 0 & 0.000000 & -1.525746 & -1.113834 \\
\hline 21 & 1 & 0 & 0.869809 & -2.194872 & -1.075147 \\
\hline 22 & 1 & 0 & -0.869809 & -2.194872 & -1.075147 \\
\hline
\end{tabular}

diiodo-1-(5,6,7,8-tetrahydro-2-naphthalenyl)borane (tetralin-5-BI 2$)\left(C_{1}\right.$ symmetry) $\mathrm{E}(\mathrm{B} 3 \mathrm{LYP} / 631 \mathrm{LAN})=-435.37995124$ hartree

\begin{tabular}{|c|c|c|c|c|c|}
\hline \multirow{2}{*}{$\begin{array}{l}\text { Center } \\
\text { Number }\end{array}$} & \multirow{2}{*}{$\begin{array}{l}\text { Atomic } \\
\text { Number }\end{array}$} & \multirow{2}{*}{$\begin{array}{l}\text { Atomic } \\
\text { Type }\end{array}$} & \multicolumn{3}{|c|}{ Coordinates (Angstroms) } \\
\hline & & & $X$ & $\mathrm{Y}$ & Z \\
\hline 1 & 5 & 0 & 0.718828 & -0.000996 & 0.298589 \\
\hline 2 & 53 & 0 & 1.737222 & 1.915386 & -0.119088 \\
\hline 3 & 53 & 0 & 1.742024 & -1.912428 & -0.129895 \\
\hline 4 & 6 & 0 & -2.272210 & -0.014204 & 2.838450 \\
\hline 5 & 6 & 0 & -3.360846 & -0.010828 & 1.975848 \\
\hline 6 & 6 & 0 & -3.195780 & -0.004340 & 0.579018 \\
\hline 7 & 6 & 0 & -1.902278 & -0.001239 & 0.049800 \\
\hline 8 & 6 & 0 & -0.805435 & -0.004692 & 0.930338 \\
\hline 9 & 6 & 0 & -0.977221 & -0.011090 & 2.308624 \\
\hline 10 & 1 & 0 & -2.427432 & -0.019212 & 3.914275 \\
\hline 11 & 1 & 0 & -4.370494 & -0.013221 & 2.383572 \\
\hline 12 & 1 & 0 & -0.112409 & -0.013645 & 2.967117 \\
\hline 13 & 6 & 0 & -2.823050 & 0.009536 & -2.413765 \\
\hline 14 & 1 & 0 & -2.725806 & -0.856669 & -3.076271 \\
\hline 15 & 1 & 0 & -2.726541 & 0.881715 & -3.068495 \\
\hline 16 & 6 & 0 & -4.265918 & 0.006306 & -1.823438 \\
\hline 17 & 1 & 0 & -4.796393 & -0.861160 & -2.229371 \\
\hline 18 & 1 & 0 & -4.796953 & 0.877222 & -2.221166 \\
\hline 19 & 6 & 0 & -4.442149 & -0.001029 & -0.283452 \\
\hline 20 & 1 & 0 & -5.046362 & -0.872405 & 0.001743 \\
\hline & & & S25 & & \\
\hline
\end{tabular}




$\begin{array}{rrrrrr}21 & 1 & 0 & -5.047353 & 0.866948 & 0.009888 \\ 22 & 6 & 0 & -1.617903 & 0.005691 & -1.438950 \\ 23 & 1 & 0 & -0.984271 & 0.876664 & -1.652538 \\ 24 & 1 & 0 & -0.983435 & -0.862691 & -1.660459\end{array}$

diiodo-2-(5,6,7,8-tetrahydro-2-naphthalenyl)borane (tetralin-6-BI $\left.\mathbf{I}_{2}\right)\left(C_{1}\right.$ symmetry) $\mathrm{E}(\mathrm{B} 3 \mathrm{LYP} / 631 \mathrm{LAN})=-435.39081494$ hartree

\begin{tabular}{|c|c|c|c|c|c|}
\hline \multirow{2}{*}{$\begin{array}{l}\text { Center } \\
\text { Number }\end{array}$} & \multirow{2}{*}{$\begin{array}{l}\text { Atomic } \\
\text { Number }\end{array}$} & \multirow{2}{*}{$\begin{array}{l}\text { Atomic } \\
\text { Type }\end{array}$} & \multicolumn{3}{|c|}{ Coordinates (Angstroms) } \\
\hline & & & $X$ & $\mathrm{Y}$ & Z \\
\hline 1 & 5 & 0 & 1.065311 & 0.000001 & 0.099974 \\
\hline 2 & 53 & 0 & 2.153707 & 1.913928 & -0.090756 \\
\hline 3 & 53 & 0 & 2.153748 & -1.913904 & -0.090738 \\
\hline 4 & 6 & 0 & -1.050450 & -0.000016 & 1.695145 \\
\hline 5 & 6 & 0 & -2.422940 & -0.000023 & 1.908436 \\
\hline 6 & 6 & 0 & -3.336176 & -0.000030 & 0.838660 \\
\hline 7 & 6 & 0 & -2.846216 & -0.000039 & -0.470221 \\
\hline 8 & 6 & 0 & -1.454987 & -0.000031 & -0.677356 \\
\hline 9 & 6 & 0 & -0.559926 & -0.000015 & 0.384760 \\
\hline 10 & 1 & 0 & -0.366161 & -0.000006 & 2.539696 \\
\hline 11 & 1 & 0 & -2.809314 & -0.000013 & 2.926448 \\
\hline 12 & 1 & 0 & -1.077902 & -0.000035 & -1.698845 \\
\hline 13 & 6 & 0 & -5.279884 & -0.000081 & -1.467391 \\
\hline 14 & 1 & 0 & -5.697537 & -0.869367 & -1.985647 \\
\hline 15 & 1 & 0 & -5.697560 & 0.869034 & -1.985916 \\
\hline 16 & 6 & 0 & -5.826424 & 0.000158 & -0.007371 \\
\hline 17 & 1 & 0 & -6.482032 & -0.868881 & 0.109385 \\
\hline 18 & 1 & 0 & -6.481621 & 0.869520 & 0.109279 \\
\hline 19 & 6 & 0 & -4.815688 & -0.000019 & 1.167822 \\
\hline 20 & 1 & 0 & -5.014051 & -0.869758 & 1.808065 \\
\hline 21 & 1 & 0 & -5.013980 & 0.869614 & 1.808231 \\
\hline 22 & 6 & 0 & -3.745902 & -0.000089 & -1.690000 \\
\hline 23 & 1 & 0 & -3.474996 & 0.869561 & -2.303207 \\
\hline 24 & 1 & 0 & -3.475008 & -0.869813 & -2.303108 \\
\hline
\end{tabular}

4,4,5,5-tetramethyl-2-(5,6,7,8-tetrahydro-2-naphthalenyl)-1,3,2-dioxaborolane

(tetralin-6-

Bpin) ( $C_{1}$ symmetry)

$\mathrm{E}(\mathrm{B} 3 \mathrm{LYP} / 6-31 \mathrm{G}(\mathrm{d}))=-798.79163616$ hartree

\begin{tabular}{|c|c|c|c|c|c|}
\hline \multirow{2}{*}{$\begin{array}{l}\text { Center } \\
\text { Number }\end{array}$} & \multirow{2}{*}{$\begin{array}{l}\text { Atomic } \\
\text { Number }\end{array}$} & \multirow{2}{*}{$\begin{array}{l}\text { Atomic } \\
\text { Type }\end{array}$} & \multicolumn{3}{|c|}{ Coordinates (Angstroms) } \\
\hline & & & $X$ & $\mathrm{Y}$ & $\mathrm{Z}$ \\
\hline 1 & 5 & 0 & -1.111263 & -0.881863 & -0.013942 \\
\hline 2 & 8 & 0 & -1.909958 & -0.530215 & -1.214142 \\
\hline 3 & 8 & 0 & -1.912961 & -0.576238 & 1.196273 \\
\hline 4 & 6 & 0 & 0.557356 & 1.082259 & 0.015009 \\
\hline 5 & 6 & 0 & 1.845314 & 1.601571 & 0.021520 \\
\hline 6 & 6 & 0 & 2.977978 & 0.769446 & 0.013793 \\
\hline 7 & 6 & 0 & 2.791524 & -0.626364 & 0.003807 \\
\hline 8 & 6 & 0 & 1.486136 & -1.134407 & -0.007496 \\
\hline 9 & 6 & 0 & 0.339074 & -0.312829 & -0.001761 \\
\hline 10 & 1 & 0 & -0.289913 & 1.759335 & 0.022195 \\
\hline 11 & 1 & 0 & 1.985150 & 2.680150 & 0.032349 \\
\hline 12 & 1 & 0 & 1.360180 & -2.214373 & -0.020975 \\
\hline & & & S26 & & \\
\hline
\end{tabular}




$\begin{array}{rrrrrr}13 & 6 & 0 & 5.298318 & -0.925252 & -0.381640 \\ 14 & 1 & 0 & 5.300092 & -0.741809 & -1.465767 \\ 15 & 1 & 0 & 6.132665 & -1.606381 & -0.174864 \\ 16 & 6 & 0 & 5.484755 & 0.406362 & 0.359046 \\ 17 & 1 & 0 & 6.460364 & 0.850585 & 0.127487 \\ 18 & 1 & 0 & 5.469258 & 0.223683 & 1.443121 \\ 19 & 6 & 0 & 4.365416 & 1.388426 & -0.018124 \\ 20 & 1 & 0 & 4.550868 & 1.767370 & -1.035943 \\ 21 & 1 & 0 & 4.388424 & 2.270699 & 0.634908 \\ 22 & 6 & 0 & 3.973952 & -1.583906 & 0.032803 \\ 23 & 1 & 0 & 4.080221 & -1.982155 & 1.054036 \\ 24 & 1 & 0 & 3.759935 & -2.450509 & -0.605647 \\ 25 & 6 & 0 & -3.270387 & -0.065630 & 0.791519 \\ 26 & 6 & 0 & -3.240758 & 0.036731 & -0.793426 \\ 27 & 6 & 0 & -3.367615 & 1.466715 & -1.386027 \\ 28 & 1 & 0 & -2.797684 & 1.513482 & -2.303791 \\ 29 & 1 & 0 & -4.404554 & 1.679041 & -1.604857 \\ 30 & 1 & 0 & -2.993470 & 2.218257 & -0.720796 \\ 31 & 6 & 0 & -4.380069 & -0.767364 & -1.462345 \\ 32 & 1 & 0 & -5.338013 & -0.373158 & -1.159818 \\ 33 & 1 & 0 & -4.287825 & -0.682416 & -2.536376 \\ 34 & 1 & 0 & -4.322302 & -1.809754 & -1.190493 \\ 35 & 6 & 0 & -3.547432 & 1.256103 & 1.544244 \\ 36 & 1 & 0 & -4.526253 & 1.628642 & 1.279834 \\ 37 & 1 & 0 & -3.515461 & 1.066569 & 2.608318 \\ 38 & 1 & 0 & -2.805960 & 2.001469 & 1.310419 \\ 39 & 6 & 0 & -4.353406 & -1.043853 & 1.304912 \\ 40 & 1 & 0 & -4.260533 & -1.141693 & 2.377594 \\ 41 & 1 & 0 & -5.337040 & -0.667077 & 1.071013 \\ 42 & 1 & 0 & -4.229415 & -2.017078 & 0.855702 \\ ----------------------------------------------------------------\end{array}$

3-chloro-4,5-dimethylbenzene (1b) ( $C_{\mathrm{s}}$ symmetry)

$\mathrm{E}(\mathrm{B} 3 \mathrm{LYP} / 6-31 \mathrm{G}(\mathrm{d}))=-770.47874745$ hartree

\begin{tabular}{|c|c|c|c|c|c|}
\hline \multirow{2}{*}{$\begin{array}{l}\text { Center } \\
\text { Number }\end{array}$} & \multirow{2}{*}{$\begin{array}{l}\text { Atomic } \\
\text { Number }\end{array}$} & \multirow{2}{*}{$\begin{array}{l}\text { Atomic } \\
\text { Type }\end{array}$} & \multicolumn{3}{|c|}{ Coordinates (Angstroms) } \\
\hline & & & $X$ & $\mathrm{Y}$ & $\mathrm{Z}$ \\
\hline 1 & 17 & 0 & 2.487501 & -0.538583 & 0.000000 \\
\hline 2 & 6 & 0 & -2.040107 & -0.665975 & 0.000000 \\
\hline 3 & 6 & 0 & -1.297032 & -1.845577 & 0.000000 \\
\hline 4 & 6 & 0 & 0.092116 & -1.785608 & 0.000000 \\
\hline 5 & 6 & 0 & 0.716721 & -0.537529 & 0.000000 \\
\hline 6 & 6 & 0 & 0.000000 & 0.667499 & 0.000000 \\
\hline 7 & 6 & 0 & -1.413224 & 0.582813 & 0.000000 \\
\hline 8 & 1 & 0 & -3.126192 & -0.712116 & 0.000000 \\
\hline 9 & 1 & 0 & -1.797040 & -2.810047 & 0.000000 \\
\hline 10 & 1 & 0 & 0.693862 & -2.687914 & 0.000000 \\
\hline 11 & 6 & 0 & -2.248862 & 1.842126 & 0.000000 \\
\hline 12 & 1 & 0 & -2.045340 & 2.465947 & 0.879894 \\
\hline 13 & 1 & 0 & -2.045340 & 2.465947 & -0.879894 \\
\hline 14 & 1 & 0 & -3.316178 & 1.602655 & 0.000000 \\
\hline 15 & 6 & 0 & 0.666720 & 2.021576 & 0.000000 \\
\hline 16 & 1 & 0 & 0.368500 & 2.606495 & -0.879295 \\
\hline 17 & 1 & 0 & 0.368500 & 2.606495 & 0.879295 \\
\hline 18 & 1 & 0 & 1.753717 & 1.942498 & 0.000000 \\
\hline
\end{tabular}


diiodo-1-(2-chloro-3,4-dimethylphenyl)borane (3-chloro-o-xylene-4-BI $\left.\mathbf{I}_{2}\right)\left(C_{1}\right.$ symmetry) $\mathrm{E}(\mathrm{B} 3 \mathrm{LYP} / 631 \mathrm{LAN})=-817.55151382$ hartree

\begin{tabular}{|c|c|c|c|c|c|}
\hline \multirow{2}{*}{$\begin{array}{l}\text { Center } \\
\text { Number }\end{array}$} & \multirow{2}{*}{$\begin{array}{l}\text { Atomic } \\
\text { Number }\end{array}$} & \multirow{2}{*}{$\begin{array}{l}\text { Atomic } \\
\text { Type }\end{array}$} & \multicolumn{3}{|c|}{ Coordinates (Angstroms) } \\
\hline & & & $\mathrm{X}$ & $\mathrm{Y}$ & $\mathrm{Z}$ \\
\hline 1 & 5 & 0 & 0.690207 & 0.000886 & -0.266132 \\
\hline 2 & 53 & 0 & 1.781486 & -1.913883 & -0.102211 \\
\hline 3 & 53 & 0 & 1.783420 & 1.913942 & -0.095257 \\
\hline 4 & 17 & 0 & -1.059274 & -0.008094 & 2.181829 \\
\hline 5 & 6 & 0 & -2.856235 & 0.007950 & -1.975831 \\
\hline 6 & 6 & 0 & -1.473159 & 0.007079 & -1.800556 \\
\hline 7 & 6 & 0 & -0.940749 & 0.002164 & -0.516094 \\
\hline 8 & 6 & 0 & -1.808195 & -0.001761 & 0.577230 \\
\hline 9 & 6 & 0 & -3.203185 & -0.000908 & 0.436582 \\
\hline 10 & 6 & 0 & -3.723361 & 0.004029 & -0.880143 \\
\hline 11 & 1 & 0 & -3.273167 & 0.011731 & -2.979755 \\
\hline 12 & 1 & 0 & -0.810151 & 0.010180 & -2.661150 \\
\hline 13 & 6 & 0 & -5.217786 & 0.005062 & -1.105649 \\
\hline 14 & 1 & 0 & -5.697434 & -0.876303 & -0.660986 \\
\hline 15 & 1 & 0 & -5.697004 & 0.884040 & -0.655800 \\
\hline 16 & 1 & 0 & -5.451554 & 0.008296 & -2.174224 \\
\hline 17 & 6 & 0 & -4.148920 & -0.005128 & 1.612851 \\
\hline 18 & 1 & 0 & -4.804889 & 0.874250 & 1.592911 \\
\hline 19 & 1 & 0 & -4.801564 & -0.886933 & 1.589323 \\
\hline 20 & 1 & 0 & -3.618102 & -0.006140 & 2.564720 \\
\hline
\end{tabular}

diiodo-2-(3-chloro-4,5-dimethylphenyl)borane (3-chloro-o-xylene-5-BI $\left.\mathbf{I}_{2}\right)\left(C_{1}\right.$ symmetry) $\mathrm{E}(\mathrm{B} 3 \mathrm{LYP} / 631 \mathrm{LAN})=-817.56099853$ hartree

\begin{tabular}{|c|c|c|c|c|c|}
\hline \multirow{2}{*}{$\begin{array}{l}\text { Center } \\
\text { Number }\end{array}$} & \multirow{2}{*}{$\begin{array}{l}\text { Atomic } \\
\text { Number }\end{array}$} & \multirow{2}{*}{$\begin{array}{l}\text { Atomic } \\
\text { Type }\end{array}$} & \multicolumn{3}{|c|}{ Coordinates (Angstroms) } \\
\hline & & & $X$ & $\mathrm{Y}$ & Z \\
\hline 1 & 5 & 0 & 0.929784 & -0.000155 & 0.046419 \\
\hline 2 & 53 & 0 & 2.031620 & -1.913557 & -0.048186 \\
\hline 3 & 53 & 0 & 2.030701 & 1.914261 & -0.037796 \\
\hline 4 & 17 & 0 & -3.830655 & 0.010642 & -2.334486 \\
\hline 5 & 6 & 0 & -1.320417 & -0.006735 & 1.435643 \\
\hline 6 & 6 & 0 & -0.714806 & -0.000912 & 0.179926 \\
\hline 7 & 6 & 0 & -1.502691 & 0.004329 & -0.965733 \\
\hline 8 & 6 & 0 & -2.892009 & 0.003587 & -0.832968 \\
\hline 9 & 6 & 0 & -3.533155 & -0.002308 & 0.413899 \\
\hline 10 & 6 & 0 & -2.711545 & -0.007484 & 1.566853 \\
\hline 11 & 1 & 0 & -0.705051 & -0.010775 & 2.331755 \\
\hline 12 & 1 & 0 & -1.057139 & 0.008971 & -1.954529 \\
\hline 13 & 6 & 0 & -3.335610 & -0.013821 & 2.943321 \\
\hline 14 & 1 & 0 & -3.972440 & 0.865164 & 3.105883 \\
\hline 15 & 1 & 0 & -3.971052 & -0.895171 & 3.098411 \\
\hline 16 & 1 & 0 & -2.566331 & -0.016544 & 3.720952 \\
\hline 17 & 6 & 0 & -5.034667 & -0.003156 & 0.567207 \\
\hline 18 & 1 & 0 & -5.372164 & -0.884958 & 1.126243 \\
\hline 19 & 1 & 0 & -5.372851 & 0.876229 & 1.129695 \\
\hline 20 & 1 & 0 & -5.544376 & -0.001392 & -0.396128 \\
\hline
\end{tabular}


$\mathrm{E}(\mathrm{B} 3 \mathrm{LYP} / 631 \mathrm{LAN})=-817.55066039$ hartree

\begin{tabular}{|c|c|c|c|c|c|}
\hline \multirow{2}{*}{$\begin{array}{l}\text { Center } \\
\text { Number }\end{array}$} & \multirow{2}{*}{$\begin{array}{l}\text { Atomic } \\
\text { Number }\end{array}$} & \multirow{2}{*}{$\begin{array}{l}\text { Atomic } \\
\text { Type }\end{array}$} & \multicolumn{3}{|c|}{ Coordinates (Angstroms) } \\
\hline & & & $\mathrm{X}$ & $\mathrm{Y}$ & Z \\
\hline 1 & 5 & 0 & 0.871319 & 0.000453 & -0.161584 \\
\hline 2 & 53 & 0 & 1.972124 & -1.913832 & -0.073024 \\
\hline 3 & 53 & 0 & 1.973013 & 1.913997 & -0.068202 \\
\hline 4 & 17 & 0 & -5.281297 & 0.002729 & -0.734960 \\
\hline 5 & 6 & 0 & -0.773079 & 0.001005 & -0.297424 \\
\hline 6 & 6 & 0 & -1.363443 & 0.005080 & -1.560388 \\
\hline 7 & 6 & 0 & -2.749241 & 0.005543 & -1.673911 \\
\hline 8 & 6 & 0 & -3.524438 & 0.001894 & -0.513355 \\
\hline 9 & 6 & 0 & -2.963391 & -0.002248 & 0.771553 \\
\hline 10 & 6 & 0 & -1.550625 & -0.002644 & 0.863551 \\
\hline 11 & 1 & 0 & -0.747198 & 0.007891 & -2.455065 \\
\hline 12 & 1 & 0 & -3.233915 & 0.008675 & -2.644136 \\
\hline 13 & 6 & 0 & -0.878348 & -0.007014 & 2.217134 \\
\hline 14 & 1 & 0 & -1.157921 & 0.871001 & 2.813553 \\
\hline 15 & 1 & 0 & -1.158049 & -0.888778 & 2.807935 \\
\hline 16 & 1 & 0 & 0.210483 & -0.006759 & 2.112468 \\
\hline 17 & 6 & 0 & -3.793577 & -0.006211 & 2.032036 \\
\hline 18 & 1 & 0 & -3.570599 & -0.887489 & 2.646735 \\
\hline 19 & 1 & 0 & -3.570471 & 0.871091 & 2.652349 \\
\hline 20 & 1 & 0 & -4.862260 & -0.005451 & 1.818184 \\
\hline
\end{tabular}

2-(3-chloro-4,5-dimethylphenyl)-4,4,5,5-tetramethyl-1,3,2-dioxaborolane (3-chloro-o-xylene-5Bpin) $\left(C_{1}\right.$ symmetry)

$\mathrm{E}(\mathrm{B} 3 \mathrm{LYP} / 6-31 \mathrm{G}(\mathrm{d}))=-1181.17213326$ hartree

\begin{tabular}{|c|c|c|c|c|c|}
\hline \multirow{2}{*}{$\begin{array}{l}\text { Center } \\
\text { Number }\end{array}$} & \multirow{2}{*}{$\begin{array}{l}\text { Atomic } \\
\text { Number }\end{array}$} & \multirow{2}{*}{$\begin{array}{l}\text { Atomic } \\
\text { Type }\end{array}$} & \multicolumn{3}{|c|}{ Coordinates (Angstroms) } \\
\hline & & & $X$ & $\mathrm{Y}$ & Z \\
\hline 1 & 17 & 0 & -4.310473 & -1.829843 & 0.014527 \\
\hline 2 & 5 & 0 & 0.876951 & -1.172843 & 0.000107 \\
\hline 3 & 8 & 0 & 1.659215 & -0.742452 & 1.232897 \\
\hline 4 & 8 & 0 & 1.663541 & -0.740939 & -1.229034 \\
\hline 5 & 6 & 0 & -2.915313 & -0.739397 & 0.003538 \\
\hline 6 & 6 & 0 & -3.091885 & 0.651462 & -0.007104 \\
\hline 7 & 6 & 0 & -1.925807 & 1.454307 & -0.015673 \\
\hline 8 & 1 & 0 & 0.221278 & 1.487644 & -0.019903 \\
\hline 9 & 1 & 0 & -1.574036 & -2.420330 & 0.014405 \\
\hline 10 & 6 & 0 & -2.042049 & 2.961128 & -0.027500 \\
\hline 11 & 1 & 0 & -2.587932 & 3.320647 & -0.909174 \\
\hline 12 & 1 & 0 & -2.583350 & 3.334821 & 0.851114 \\
\hline 13 & 1 & 0 & -1.053384 & 3.429114 & -0.033813 \\
\hline 14 & 6 & 0 & -4.450668 & 1.308534 & -0.009844 \\
\hline 15 & 1 & 0 & -4.573949 & 1.960046 & 0.864418 \\
\hline 16 & 1 & 0 & -4.579248 & 1.941846 & -0.896671 \\
\hline 17 & 1 & 0 & -5.258822 & 0.577363 & 0.000069 \\
\hline 18 & 6 & 0 & 2.977759 & -0.136628 & -0.756062 \\
\hline 19 & 6 & 0 & 2.944534 & -0.075385 & 0.764320 \\
\hline 20 & 6 & 0 & -0.663216 & 0.855714 & -0.013234 \\
\hline 21 & 6 & 0 & -0.523063 & -0.531319 & -0.002447 \\
\hline 22 & 6 & 0 & -1.654954 & -1.338833 & 0.006001 \\
\hline 23 & 6 & 0 & 2.994337 & 1.387317 & 1.243510 \\
\hline 24 & 1 & 0 & 2.408891 & 1.491337 & 2.133079 \\
\hline & & & S29 & & \\
\hline
\end{tabular}




$\begin{array}{lllrrr}25 & 1 & 0 & 4.008052 & 1.660775 & 1.449673 \\ 26 & 1 & 0 & 2.600673 & 2.026130 & 0.480722 \\ 27 & 6 & 0 & 3.172107 & 1.270570 & -1.350724 \\ 28 & 1 & 0 & 4.098255 & 1.679385 & -1.004287 \\ 29 & 1 & 0 & 3.187412 & 1.207449 & -2.418751 \\ 30 & 1 & 0 & 2.365686 & 1.902603 & -1.042308 \\ 31 & 6 & 0 & 4.163753 & -0.996527 & -1.231027 \\ 32 & 1 & 0 & 4.140789 & -1.077786 & -2.297690 \\ 33 & 1 & 0 & 5.081352 & -0.537086 & -0.927999 \\ 34 & 1 & 0 & 4.093153 & -1.972172 & -0.797400 \\ 35 & 6 & 0 & 4.172242 & -0.789201 & 1.359970 \\ 36 & 1 & 0 & 5.065569 & -0.331044 & 0.989890 \\ 37 & 1 & 0 & 4.147177 & -0.711522 & 2.426852 \\ 38 & 1 & 0 & 4.156999 & -1.821001 & 1.077028\end{array}$

naphthalene (1c) $\left(D_{2 \mathrm{~h}}\right.$ symmetry)

$\mathrm{E}(\mathrm{B} 3 \mathrm{LYP} / 6-31 \mathrm{G}(\mathrm{d}))=-385.89272966$ hartree

\begin{tabular}{|c|c|c|c|c|c|}
\hline \multirow{2}{*}{$\begin{array}{l}\text { Center } \\
\text { Number }\end{array}$} & \multirow{2}{*}{$\begin{array}{l}\text { Atomic } \\
\text { Number }\end{array}$} & \multirow{2}{*}{$\begin{array}{l}\text { Atomic } \\
\text { Type }\end{array}$} & \multicolumn{3}{|c|}{ Coordinates (Angstroms) } \\
\hline & & & $X$ & $\mathrm{Y}$ & $\mathrm{Z}$ \\
\hline 1 & 6 & 0 & 2.426104 & -0.708815 & 0.000060 \\
\hline 2 & 6 & 0 & 1.241200 & -1.402057 & -0.000132 \\
\hline 3 & 6 & 0 & -0.000592 & -0.709186 & -0.000027 \\
\hline 4 & 6 & 0 & 0.000990 & 0.709681 & -0.000089 \\
\hline 5 & 6 & 0 & 1.242689 & 1.402120 & -0.000049 \\
\hline 6 & 6 & 0 & 2.427242 & 0.707212 & 0.000133 \\
\hline 7 & 1 & 0 & -1.233064 & -2.501900 & -0.000215 \\
\hline 8 & 1 & 0 & 3.387976 & -1.242474 & 0.000059 \\
\hline 9 & 1 & 0 & 1.230581 & -2.502338 & -0.000143 \\
\hline 10 & 6 & 0 & -1.242645 & -1.401635 & 0.000042 \\
\hline 11 & 6 & 0 & -1.241303 & 1.401800 & -0.000099 \\
\hline 12 & 1 & 0 & 1.233044 & 2.502286 & 0.000106 \\
\hline 13 & 1 & 0 & 3.389685 & 1.239752 & 0.000180 \\
\hline 14 & 6 & 0 & -2.426293 & 0.708587 & -0.000018 \\
\hline 15 & 6 & 0 & -2.427269 & -0.707638 & 0.000134 \\
\hline 16 & 1 & 0 & -1.231082 & 2.502102 & -0.000121 \\
\hline 17 & 1 & 0 & -3.388124 & 1.242252 & 0.000241 \\
\hline 18 & 1 & 0 & -3.389754 & -1.240089 & 0.000166 \\
\hline
\end{tabular}

diiodo-1-naphthalenylborane (naphthalene-1-BI 2$)\left(C_{1}\right.$ symmetry)

$\mathrm{E}(\mathrm{B} 3 \mathrm{LYP} / 631 \mathrm{LAN})=-432.96480512$ hartree

\begin{tabular}{rrrrrr}
$\begin{array}{c}\text { Center } \\
\text { Number }\end{array}$ & $\begin{array}{c}\text { Atomic } \\
\text { Number }\end{array}$ & $\begin{array}{c}\text { Atomic } \\
\text { Type }\end{array}$ & \multicolumn{2}{c}{ Coordinates (Angstroms) } \\
-1 & 5 & 0 & X & Z \\
2 & 53 & 0 & 1.672032 & 0.000000 & 0.269741 \\
3 & 53 & 0 & 1.672032 & -1.913916 & -0.097346 \\
4 & 6 & 0 & -4.118761 & 0.000002 & -1.917347 \\
5 & 6 & 0 & -4.376485 & 0.000002 & -0.540455 \\
6 & 6 & 0 & -3.312399 & 0.000000 & 0.371489 \\
7 & 6 & 0 & -1.990589 & 0.000000 & -0.094064 \\
8 & 6 & 0 & -1.732865 & -0.000002 & -1.471562 \\
9 & 6 & 0 & -2.796951 & -0.000001 & -2.383506 \\
10 & 1 & 0 & -4.579355 & -0.000004 & 2.104447 \\
& & & S30 & &
\end{tabular}




\begin{tabular}{rlrrrr}
11 & 1 & 0 & -4.931214 & 0.000006 & -2.614243 \\
12 & 1 & 0 & -5.385717 & 0.000002 & -0.184995 \\
13 & 6 & 0 & -3.570123 & -0.000002 & 1.748987 \\
14 & 6 & 0 & -0.926503 & 0.000000 & 0.817880 \\
15 & 1 & 0 & -0.723634 & -0.000005 & -1.827022 \\
16 & 1 & 0 & -2.600173 & -0.000001 & -3.435257 \\
17 & 6 & 0 & -1.184227 & -0.000001 & 2.195378 \\
18 & 6 & 0 & -2.506037 & -0.000003 & 2.660932 \\
19 & 1 & 0 & -0.371774 & 0.000001 & 2.891668 \\
20 & 1 & 0 & -2.702815 & -0.000004 & 3.712682 \\
\hline
\end{tabular}

diiodo-2-naphthalenylborane (naphthalene-2-BI 2$)\left(C_{1}\right.$ symmetry) $\mathrm{E}(\mathrm{B} 3 \mathrm{LYP} / 631 \mathrm{LAN})=-432.96632174$ hartree

\begin{tabular}{|c|c|c|c|c|c|}
\hline \multirow{2}{*}{$\begin{array}{l}\text { Center } \\
\text { Number }\end{array}$} & \multirow{2}{*}{$\begin{array}{l}\text { Atomic } \\
\text { Number }\end{array}$} & \multirow{2}{*}{$\begin{array}{c}\text { Atomic } \\
\text { Type }\end{array}$} & \multicolumn{3}{|c|}{ Coordinates (Angstroms) } \\
\hline & & & $X$ & $\mathrm{Y}$ & $\mathrm{Z}$ \\
\hline 1 & 5 & 0 & 0.930897 & 0.000000 & 0.088830 \\
\hline 2 & 53 & 0 & 2.022584 & 1.913917 & -0.082174 \\
\hline 3 & 53 & 0 & 2.022586 & -1.913916 & -0.082174 \\
\hline 4 & 6 & 0 & -5.732853 & 0.000000 & -0.095796 \\
\hline 5 & 6 & 0 & -4.852777 & 0.000000 & 0.994795 \\
\hline 6 & 6 & 0 & -3.468259 & -0.000001 & 0.777922 \\
\hline 7 & 6 & 0 & -2.963819 & -0.000001 & -0.529542 \\
\hline 8 & 6 & 0 & -3.843895 & -0.000002 & -1.620133 \\
\hline 9 & 6 & 0 & -5.228412 & -0.000001 & -1.403259 \\
\hline 10 & 1 & 0 & -2.973335 & 0.000001 & 2.866790 \\
\hline 11 & 1 & 0 & -6.789963 & 0.000002 & 0.069792 \\
\hline 12 & 1 & 0 & -5.237928 & 0.000002 & 1.993072 \\
\hline 13 & 6 & 0 & -2.588183 & 0.000000 & 1.868512 \\
\hline 14 & 6 & 0 & -1.579301 & -0.000002 & -0.746415 \\
\hline 15 & 1 & 0 & -3.458743 & -0.000002 & -2.618410 \\
\hline 16 & 1 & 0 & -5.900370 & -0.000001 & -2.235949 \\
\hline 17 & 6 & 0 & -0.699225 & -0.000001 & 0.344175 \\
\hline 18 & 6 & 0 & -1.203666 & -0.000001 & 1.651639 \\
\hline 19 & 1 & 0 & -1.194149 & -0.000002 & -1.744693 \\
\hline 20 & 1 & 0 & -0.531708 & -0.000002 & 2.484329 \\
\hline
\end{tabular}

\section{4,4,5,5-tetramethyl-2-(2-naphthalenyl)-1,3,2-dioxaborolane (naphthalene-2-Bpin)}

$\left(C_{1}\right.$ symmetry)

$\mathrm{E}(\mathrm{B} 3 \mathrm{LYP} / 6-31 \mathrm{G}(\mathrm{d}))=-796.58574167$ hartree

\begin{tabular}{|c|c|c|c|c|c|}
\hline \multirow{2}{*}{$\begin{array}{l}\text { Center } \\
\text { Number }\end{array}$} & \multirow{2}{*}{$\begin{array}{l}\text { Atomic } \\
\text { Number }\end{array}$} & \multirow{2}{*}{$\begin{array}{l}\text { Atomic } \\
\text { Type }\end{array}$} & \multicolumn{3}{|c|}{ Coordinates (Angstroms) } \\
\hline & & & $\mathrm{X}$ & $\mathrm{Y}$ & $\mathrm{Z}$ \\
\hline 1 & 5 & 0 & 0.923948 & -1.084534 & 0.020924 \\
\hline 2 & 8 & 0 & 1.709422 & -0.675840 & -1.216837 \\
\hline 3 & 8 & 0 & 1.706333 & -0.629232 & 1.244656 \\
\hline 4 & 6 & 0 & -0.601981 & 0.966195 & -0.026840 \\
\hline 5 & 6 & 0 & -1.847497 & 1.552322 & -0.040018 \\
\hline 6 & 6 & 0 & -3.026836 & 0.759673 & -0.020515 \\
\hline 7 & 6 & 0 & -2.900187 & -0.668091 & 0.013286 \\
\hline 8 & 6 & 0 & -1.599692 & -1.240883 & 0.026110 \\
\hline 9 & 6 & 0 & -0.476808 & -0.444787 & 0.006564 \\
\hline
\end{tabular}




\begin{tabular}{|c|c|c|c|c|c|}
\hline 10 & 1 & 0 & -4.420687 & 2.415837 & -0.058997 \\
\hline 11 & 1 & 0 & 0.291575 & 1.584498 & -0.042033 \\
\hline 12 & 1 & 0 & -1.946324 & 2.635205 & -0.065651 \\
\hline 13 & 6 & 0 & -4.327334 & 1.332468 & -0.033341 \\
\hline 14 & 6 & 0 & -4.079526 & -1.460751 & 0.032787 \\
\hline 15 & 1 & 0 & -1.506329 & -2.324252 & 0.051762 \\
\hline 16 & 6 & 0 & -5.325036 & -0.874620 & 0.019612 \\
\hline 17 & 6 & 0 & -5.450210 & 0.536362 & -0.013789 \\
\hline 18 & 1 & 0 & -3.980686 & -2.543631 & 0.058418 \\
\hline 19 & 1 & 0 & -6.218605 & -1.492908 & 0.034809 \\
\hline 20 & 1 & 0 & -6.438678 & 0.987802 & -0.023920 \\
\hline 21 & 6 & 0 & 3.023178 & -0.060894 & -0.756457 \\
\hline 22 & 6 & 0 & 2.990645 & 0.030020 & 0.762454 \\
\hline 23 & 6 & 0 & 3.215601 & 1.334632 & -1.378615 \\
\hline 24 & 1 & 0 & 4.261065 & 1.542086 & -1.472782 \\
\hline 25 & 1 & 0 & 2.758399 & 1.360125 & -2.345680 \\
\hline 26 & 1 & 0 & 2.761036 & 2.071304 & -0.749659 \\
\hline 27 & 6 & 0 & 3.038999 & 1.501865 & 1.212935 \\
\hline 28 & 1 & 0 & 2.870422 & 1.557636 & 2.268099 \\
\hline 29 & 1 & 0 & 3.999315 & 1.913905 & 0.982897 \\
\hline 30 & 1 & 0 & 2.280856 & 2.056700 & 0.700804 \\
\hline 31 & 6 & 0 & 4.219476 & -0.670610 & 1.371300 \\
\hline 32 & 1 & 0 & 5.110389 & -0.177152 & 1.043164 \\
\hline 33 & 1 & 0 & 4.161250 & -0.628948 & 2.438902 \\
\hline 34 & 1 & 0 & 4.240586 & -1.692530 & 1.054863 \\
\hline 35 & 6 & 0 & 4.209926 & -0.928555 & -1.215144 \\
\hline 36 & 1 & 0 & 4.202599 & -1.007523 & -2.282201 \\
\hline 37 & 1 & 0 & 5.126151 & -0.476483 & -0.897241 \\
\hline 38 & 1 & 0 & 4.125587 & -1.904513 & -0.784689 \\
\hline
\end{tabular}

anthracene (1d) ( $D_{2 \mathrm{~h}}$ symmetry)

$\mathrm{E}(\mathrm{B} 3 \mathrm{LYP} / 6-31 \mathrm{G}(\mathrm{d}))=-539.53052354$ hartree

\begin{tabular}{|c|c|c|c|c|c|}
\hline \multirow{2}{*}{$\begin{array}{l}\text { Center } \\
\text { Number }\end{array}$} & \multirow{2}{*}{$\begin{array}{l}\text { Atomic } \\
\text { Number }\end{array}$} & \multirow{2}{*}{$\begin{array}{l}\text { Atomic } \\
\text { Type }\end{array}$} & \multicolumn{3}{|c|}{ Coordinates (Angstroms) } \\
\hline & & & $\mathrm{X}$ & Y & $\mathrm{Z}$ \\
\hline 1 & 6 & 0 & 0.000000 & 3.660680 & 0.713146 \\
\hline 2 & 6 & 0 & 0.000000 & 2.479530 & 1.407037 \\
\hline 3 & 6 & 0 & 0.000000 & 1.223923 & 0.722624 \\
\hline 4 & 6 & 0 & 0.000000 & 1.223923 & -0.722624 \\
\hline 5 & 6 & 0 & 0.000000 & 2.479530 & -1.407037 \\
\hline 6 & 6 & 0 & 0.000000 & 3.660680 & -0.713146 \\
\hline 7 & 6 & 0 & 0.000000 & 0.000000 & 1.403377 \\
\hline 8 & 6 & 0 & 0.000000 & 0.000000 & -1.403377 \\
\hline 9 & 6 & 0 & 0.000000 & -1.223923 & -0.722624 \\
\hline 10 & 6 & 0 & 0.000000 & -1.223923 & 0.722624 \\
\hline 11 & 6 & 0 & 0.000000 & -2.479530 & 1.407037 \\
\hline 12 & 1 & 0 & 0.000000 & -2.476865 & 2.494580 \\
\hline 13 & 6 & 0 & 0.000000 & -3.660680 & 0.713146 \\
\hline 14 & 6 & 0 & 0.000000 & -3.660680 & -0.713146 \\
\hline 15 & 6 & 0 & 0.000000 & -2.479530 & -1.407037 \\
\hline 16 & 1 & 0 & 0.000000 & 0.000000 & 2.491747 \\
\hline 17 & 1 & 0 & 0.000000 & 4.607360 & 1.246657 \\
\hline 18 & 1 & 0 & 0.000000 & 2.476865 & 2.494580 \\
\hline 19 & 1 & 0 & 0.000000 & 2.476865 & -2.494580 \\
\hline \multirow[t]{2}{*}{20} & 1 & 0 & 0.000000 & 4.607360 & -1.246657 \\
\hline & & & S32 & & \\
\hline
\end{tabular}




$\begin{array}{rrrrrr}21 & 1 & 0 & 0.000000 & 0.000000 & -2.491747 \\ 22 & 1 & 0 & 0.000000 & -4.607360 & 1.246657 \\ 23 & 1 & 0 & 0.000000 & -4.607360 & -1.246657 \\ 24 & 1 & 0 & 0.000000 & -2.476865 & -2.494580\end{array}$

diiodo-1-anthracenylborane (anthracene-1-BI $)\left(C_{1}\right.$ symmetry)

$\mathrm{E}(\mathrm{B} 3 \mathrm{LYP} / 631 \mathrm{LAN})=-586.60276127$ hartree

\begin{tabular}{|c|c|c|c|c|c|}
\hline \multirow{2}{*}{$\begin{array}{l}\text { Center } \\
\text { Number }\end{array}$} & \multirow{2}{*}{$\begin{array}{l}\text { Atomic } \\
\text { Number }\end{array}$} & \multirow{2}{*}{$\begin{array}{c}\text { Atomic } \\
\text { Type }\end{array}$} & \multicolumn{3}{|c|}{ Coordinates (Angstroms) } \\
\hline & & & $\mathrm{X}$ & $\mathrm{Y}$ & $\mathrm{Z}$ \\
\hline 1 & 5 & 0 & 1.385996 & 0.274049 & -0.000001 \\
\hline 2 & 53 & 0 & 2.195560 & -0.478039 & -1.913915 \\
\hline 3 & 53 & 0 & 2.195561 & -0.478030 & 1.913917 \\
\hline 4 & 6 & 0 & 0.469408 & 2.735421 & -0.000005 \\
\hline 5 & 6 & 0 & 0.177143 & 1.397070 & -0.000003 \\
\hline 6 & 6 & 0 & -1.177756 & 0.939668 & -0.000002 \\
\hline 7 & 6 & 0 & -2.239007 & 1.920734 & -0.000003 \\
\hline 8 & 6 & 0 & -1.889239 & 3.307325 & -0.000006 \\
\hline 9 & 6 & 0 & -0.577923 & 3.703618 & -0.000007 \\
\hline 10 & 6 & 0 & -1.508703 & -0.421172 & 0.000001 \\
\hline 11 & 6 & 0 & -3.569711 & 1.484114 & -0.000002 \\
\hline 12 & 6 & 0 & -3.900658 & 0.123274 & 0.000000 \\
\hline 13 & 6 & 0 & -2.839407 & -0.857792 & 0.000002 \\
\hline 14 & 6 & 0 & -3.189175 & -2.244383 & 0.000005 \\
\hline 15 & 1 & 0 & -2.388780 & -2.980674 & 0.000006 \\
\hline 16 & 6 & 0 & -4.500491 & -2.640676 & 0.000005 \\
\hline 17 & 6 & 0 & -5.547821 & -1.672479 & 0.000004 \\
\hline 18 & 6 & 0 & -5.255557 & -0.334129 & 0.000001 \\
\hline 19 & 1 & 0 & -0.709509 & -1.159981 & 0.000002 \\
\hline 20 & 1 & 0 & 1.503794 & 3.068412 & -0.000006 \\
\hline 21 & 1 & 0 & -2.689634 & 4.043616 & -0.000007 \\
\hline 22 & 1 & 0 & -0.327055 & 4.760928 & -0.000009 \\
\hline 23 & 1 & 0 & -4.368905 & 2.222923 & -0.000004 \\
\hline 24 & 1 & 0 & -4.751359 & -3.697986 & 0.000007 \\
\hline 25 & 1 & 0 & -6.582208 & -2.005470 & 0.000004 \\
\hline 26 & 1 & 0 & -6.052335 & 0.406076 & 0.000000 \\
\hline
\end{tabular}

diiodo-2-anthracenylborane (anthracene-2-BI $)\left(C_{1}\right.$ symmetry)

$\mathrm{E}(\mathrm{B} 3 \mathrm{LYP} / 631 \mathrm{LAN})=-586.61406243$ hartree

\begin{tabular}{|c|c|c|c|c|c|}
\hline \multirow{2}{*}{$\begin{array}{l}\text { Center } \\
\text { Number }\end{array}$} & \multirow{2}{*}{$\begin{array}{l}\text { Atomic } \\
\text { Number }\end{array}$} & \multirow{2}{*}{$\begin{array}{l}\text { Atomic } \\
\text { Type }\end{array}$} & \multicolumn{3}{|c|}{ Coordinates (Angstroms) } \\
\hline & & & $X$ & $\mathrm{Y}$ & $\mathrm{Z}$ \\
\hline 1 & 5 & 0 & 1.891418 & -0.000020 & 0.086366 \\
\hline 2 & 53 & 0 & 2.963379 & 1.914034 & -0.180830 \\
\hline 3 & 53 & 0 & 2.963664 & -1.913798 & -0.181671 \\
\hline 4 & 6 & 0 & -6.768350 & 0.000616 & -1.456804 \\
\hline & & & S33 & & \\
\hline
\end{tabular}




\begin{tabular}{rrrrrr}
5 & 6 & 0 & -5.445415 & 0.000799 & -1.812391 \\
6 & 6 & 0 & -4.416435 & 0.000340 & -0.819330 \\
7 & 6 & 0 & -4.799943 & -0.000325 & 0.574106 \\
8 & 6 & 0 & -6.192151 & -0.000495 & 0.900797 \\
9 & 6 & 0 & -7.146828 & -0.000040 & -0.081645 \\
10 & 6 & 0 & -3.055747 & 0.000512 & -1.150900 \\
11 & 6 & 0 & -3.800541 & -0.000778 & 1.555231 \\
12 & 6 & 0 & -2.439853 & -0.000606 & 1.223661 \\
13 & 6 & 0 & -2.056345 & 0.000058 & -0.169776 \\
14 & 6 & 0 & -0.664137 & 0.000228 & -0.496467 \\
15 & 1 & 0 & -0.378118 & 0.000728 & -1.545728 \\
16 & 6 & 0 & 0.290539 & -0.000227 & 0.485976 \\
17 & 6 & 0 & -0.087938 & -0.000882 & 1.861134 \\
18 & 6 & 0 & -1.410873 & -0.001065 & 2.216722 \\
19 & 1 & 0 & -2.766940 & 0.001012 & -2.200253 \\
20 & 1 & 0 & -7.539520 & 0.000970 & -2.222398 \\
21 & 1 & 0 & -5.154258 & 0.001298 & -2.860239 \\
22 & 1 & 0 & -6.478170 & -0.000995 & 1.950059 \\
23 & 1 & 0 & -8.201141 & -0.000176 & 0.181531 \\
24 & 1 & 0 & -4.089348 & -0.001278 & 2.604583 \\
25 & 1 & 0 & 0.683232 & -0.001237 & 2.626728 \\
26 & 1 & 0 & -1.702030 & -0.001565 & 3.264570 \\
\hline-----------------------------------------------------------------------------------------------------
\end{tabular}

diiodo-9-anthracenylborane (anthracene-9-BI $)\left(C_{1}\right.$ symmetry)

$\mathrm{E}(\mathrm{B} 3 \mathrm{LYP} / 631 \mathrm{LAN})=-586.60063495$ hartree

\begin{tabular}{|c|c|c|c|c|c|}
\hline \multirow{2}{*}{$\begin{array}{l}\text { Center } \\
\text { Number }\end{array}$} & \multirow{2}{*}{$\begin{array}{l}\text { Atomic } \\
\text { Number }\end{array}$} & \multirow{2}{*}{$\begin{array}{l}\text { Atomic } \\
\text { Type }\end{array}$} & \multicolumn{3}{|c|}{ Coordinates (Angstroms) } \\
\hline & & & $\mathrm{X}$ & $\mathrm{Y}$ & Z \\
\hline 1 & 5 & 0 & 0.000000 & 0.000000 & -0.830028 \\
\hline 2 & 53 & 0 & 1.913916 & 0.000371 & -1.935028 \\
\hline 3 & 53 & 0 & -1.913916 & -0.000371 & -1.935028 \\
\hline 4 & 6 & 0 & 0.000000 & -3.660680 & 1.510203 \\
\hline 5 & 6 & 0 & 0.000000 & -2.479530 & 0.816312 \\
\hline 6 & 6 & 0 & 0.000000 & -1.223923 & 1.500724 \\
\hline 7 & 6 & 0 & 0.000000 & -1.223923 & 2.945973 \\
\hline 8 & 6 & 0 & 0.000000 & -2.479530 & 3.630385 \\
\hline 9 & 6 & 0 & 0.000000 & -3.660680 & 2.936494 \\
\hline 10 & 6 & 0 & 0.000000 & 0.000000 & 0.819972 \\
\hline 11 & 6 & 0 & 0.000000 & 0.000000 & 3.626725 \\
\hline 12 & 6 & 0 & 0.000000 & 1.223923 & 2.945973 \\
\hline 13 & 6 & 0 & 0.000000 & 1.223923 & 1.500724 \\
\hline 14 & 6 & 0 & 0.000000 & 2.479530 & 0.816312 \\
\hline 15 & 1 & 0 & 0.000000 & 2.476866 & -0.271231 \\
\hline 16 & 6 & 0 & 0.000000 & 3.660680 & 1.510203 \\
\hline 17 & 6 & 0 & 0.000000 & 3.660680 & 2.936494 \\
\hline 18 & 6 & 0 & 0.000000 & 2.479530 & 3.630385 \\
\hline 19 & 1 & 0 & 0.000000 & -4.607360 & 0.976691 \\
\hline 20 & 1 & 0 & 0.000000 & -2.476866 & -0.271231 \\
\hline 21 & 1 & 0 & 0.000000 & -2.476866 & 4.717928 \\
\hline 22 & 1 & 0 & 0.000000 & -4.607360 & 3.470006 \\
\hline 23 & 1 & 0 & 0.000000 & 0.000000 & 4.715096 \\
\hline 24 & 1 & 0 & 0.000000 & 4.607360 & 0.976691 \\
\hline 25 & 1 & 0 & 0.000000 & 4.607360 & 3.470006 \\
\hline \multirow[t]{2}{*}{26} & 1 & 0 & 0.000000 & 2.476866 & 4.717928 \\
\hline & & & S34 & & \\
\hline
\end{tabular}


2-(2-anthracenyl)-4,4,5,5-tetramethyl-1,3,2-dioxaborolane (anthracene-2-Bpin) $\left(C_{1}\right.$ symmetry) $\mathrm{E}(\mathrm{B} 3 \mathrm{LYP} / 6-31 \mathrm{G}(\mathrm{d}))=-950.22369898$ hartree

\begin{tabular}{|c|c|c|c|c|c|}
\hline \multirow{2}{*}{$\begin{array}{l}\text { Center } \\
\text { Number }\end{array}$} & \multirow{2}{*}{$\begin{array}{l}\text { Atomic } \\
\text { Number }\end{array}$} & \multirow{2}{*}{$\begin{array}{l}\text { Atomic } \\
\text { Type }\end{array}$} & \multicolumn{3}{|c|}{ Coordinates (Angstroms) } \\
\hline & & & $\mathrm{X}$ & Y & $\mathrm{Z}$ \\
\hline 1 & 5 & 0 & 2.088562 & -1.078779 & 0.013206 \\
\hline 2 & 8 & 0 & 2.881481 & -0.675661 & -1.221633 \\
\hline 3 & 8 & 0 & 2.879214 & -0.646969 & 1.240134 \\
\hline 4 & 6 & 0 & -1.730917 & -0.583727 & 0.006835 \\
\hline 5 & 6 & 0 & -0.432796 & -1.183542 & 0.015406 \\
\hline 6 & 6 & 0 & 0.699879 & -0.413146 & 0.003682 \\
\hline 7 & 6 & 0 & -3.092760 & 1.456459 & -0.023097 \\
\hline 8 & 6 & 0 & -2.907140 & -1.343843 & 0.018418 \\
\hline 9 & 6 & 0 & -4.173404 & -0.745584 & 0.009862 \\
\hline 10 & 6 & 0 & -4.268983 & 0.696342 & -0.011515 \\
\hline 11 & 6 & 0 & -5.567104 & 1.296158 & -0.020085 \\
\hline 12 & 1 & 0 & -5.636368 & 2.381377 & -0.036174 \\
\hline 13 & 6 & 0 & -6.699779 & 0.525761 & -0.008361 \\
\hline 14 & 6 & 0 & -6.605453 & -0.897251 & 0.012735 \\
\hline 15 & 6 & 0 & -5.381000 & -1.511447 & 0.021538 \\
\hline 16 & 1 & 0 & -3.164738 & 2.542327 & -0.039195 \\
\hline 17 & 1 & 0 & 1.514878 & 1.604749 & -0.026477 \\
\hline 18 & 1 & 0 & -0.693482 & 2.708929 & -0.042300 \\
\hline 19 & 1 & 0 & -0.363532 & -2.268761 & 0.031495 \\
\hline 20 & 1 & 0 & -2.835162 & -2.429711 & 0.034516 \\
\hline 21 & 1 & 0 & -7.679669 & 0.995450 & -0.015082 \\
\hline 22 & 1 & 0 & -7.514778 & -1.492133 & 0.021797 \\
\hline 23 & 1 & 0 & -5.306418 & -2.596313 & 0.037621 \\
\hline 24 & 6 & 0 & 4.206379 & -0.088496 & -0.756935 \\
\hline 25 & 6 & 0 & 4.175509 & -0.008083 & 0.762603 \\
\hline 26 & 6 & 0 & 0.605553 & 1.009867 & -0.017415 \\
\hline 27 & 6 & 0 & -0.618900 & 1.624062 & -0.026218 \\
\hline 28 & 6 & 0 & -1.826496 & 0.858200 & -0.014542 \\
\hline 29 & 6 & 0 & 5.376883 & -0.974588 & -1.222082 \\
\hline 30 & 1 & 0 & 5.364407 & -1.050716 & -2.289297 \\
\hline 31 & 1 & 0 & 6.301633 & -0.538609 & -0.906394 \\
\hline 32 & 1 & 0 & 5.277879 & -1.950099 & -0.793741 \\
\hline 33 & 6 & 0 & 4.424591 & 1.307736 & -1.368915 \\
\hline 34 & 1 & 0 & 5.468941 & 1.540540 & -1.362914 \\
\hline 35 & 1 & 0 & 4.063055 & 1.316239 & -2.375950 \\
\hline 36 & 1 & 0 & 3.893393 & 2.036537 & -0.793088 \\
\hline 37 & 6 & 0 & 4.251071 & 1.459289 & 1.223807 \\
\hline 38 & 1 & 0 & 4.057083 & 1.513301 & 2.274688 \\
\hline 39 & 1 & 0 & 5.227476 & 1.847085 & 1.020959 \\
\hline 40 & 1 & 0 & 3.521154 & 2.037020 & 0.696219 \\
\hline 41 & 6 & 0 & 5.391160 & -0.735746 & 1.366174 \\
\hline 42 & 1 & 0 & 6.292011 & -0.292558 & 0.996105 \\
\hline 43 & 1 & 0 & 5.364061 & -0.651375 & 2.432499 \\
\hline 44 & 1 & 0 & 5.362048 & -1.768889 & 1.089284 \\
\hline
\end{tabular}

9,10-diphenylanthracene (1e) $\left(D_{2 h}\right.$ symmetry) $\mathrm{E}(\mathrm{B} 3 \mathrm{LYP} / 6-31 \mathrm{G}(\mathrm{d}))=-1001.63080377$ hartree 


\begin{tabular}{|c|c|c|c|c|c|}
\hline \multirow{2}{*}{$\begin{array}{l}\text { Center } \\
\text { Number }\end{array}$} & \multirow{2}{*}{$\begin{array}{l}\text { Atomic } \\
\text { Number }\end{array}$} & \multirow{2}{*}{$\begin{array}{l}\text { Atomic } \\
\text { Type }\end{array}$} & \multicolumn{3}{|c|}{ Coordinates (Angstroms) } \\
\hline & & & $\mathrm{X}$ & $\mathrm{Y}$ & $\mathrm{Z}$ \\
\hline 1 & 6 & 0 & -0.711233 & 3.667373 & 0.000000 \\
\hline 2 & 6 & 0 & -1.402071 & 2.485081 & 0.000000 \\
\hline 3 & 6 & 0 & -0.723372 & 1.222941 & 0.000000 \\
\hline 4 & 6 & 0 & 0.723372 & 1.222941 & 0.000000 \\
\hline 5 & 6 & 0 & 1.402071 & 2.485081 & 0.000000 \\
\hline 6 & 6 & 0 & 0.711233 & 3.667373 & 0.000000 \\
\hline 7 & 6 & 0 & -1.428737 & 0.000000 & 0.000000 \\
\hline 8 & 6 & 0 & 1.428737 & 0.000000 & 0.000000 \\
\hline 9 & 6 & 0 & 0.723372 & -1.222941 & 0.000000 \\
\hline 10 & 6 & 0 & -0.723372 & -1.222941 & 0.000000 \\
\hline 11 & 6 & 0 & -1.402071 & -2.485081 & 0.000000 \\
\hline 12 & 1 & 0 & -2.486450 & -2.490018 & 0.000000 \\
\hline 13 & 6 & 0 & -0.711233 & -3.667373 & 0.000000 \\
\hline 14 & 6 & 0 & 0.711233 & -3.667373 & 0.000000 \\
\hline 15 & 6 & 0 & 1.402071 & -2.485081 & 0.000000 \\
\hline 16 & 1 & 0 & -1.249760 & 4.611352 & 0.000000 \\
\hline 17 & 1 & 0 & -2.486450 & 2.490018 & 0.000000 \\
\hline 18 & 1 & 0 & 2.486450 & 2.490018 & 0.000000 \\
\hline 19 & 1 & 0 & 1.249760 & 4.611352 & 0.000000 \\
\hline 20 & 1 & 0 & -1.249760 & -4.611352 & 0.000000 \\
\hline 21 & 1 & 0 & 1.249760 & -4.611352 & 0.000000 \\
\hline 22 & 1 & 0 & 2.486450 & -2.490018 & 0.000000 \\
\hline 23 & 6 & 0 & 2.927236 & 0.000000 & 0.000000 \\
\hline 24 & 6 & 0 & 3.644007 & 0.000000 & 1.206167 \\
\hline 25 & 6 & 0 & 3.644007 & 0.000000 & -1.206167 \\
\hline 26 & 6 & 0 & 5.039904 & 0.000000 & 1.206704 \\
\hline 27 & 1 & 0 & 3.099827 & 0.000000 & 2.146911 \\
\hline 28 & 6 & 0 & 5.039904 & 0.000000 & -1.206704 \\
\hline 29 & 1 & 0 & 3.099827 & 0.000000 & -2.146911 \\
\hline 30 & 6 & 0 & 5.741838 & 0.000000 & 0.000000 \\
\hline 31 & 1 & 0 & 5.578411 & 0.000000 & 2.150897 \\
\hline 32 & 1 & 0 & 5.578411 & 0.000000 & -2.150897 \\
\hline 33 & 1 & 0 & 6.828619 & 0.000000 & 0.000000 \\
\hline 34 & 6 & 0 & -2.927236 & 0.000000 & 0.000000 \\
\hline 35 & 6 & 0 & -3.644007 & 0.000000 & -1.206167 \\
\hline 36 & 6 & 0 & -3.644007 & 0.000000 & 1.206167 \\
\hline 37 & 6 & 0 & -5.039904 & 0.000000 & -1.206704 \\
\hline 38 & 1 & 0 & -3.099827 & 0.000000 & -2.146911 \\
\hline 39 & 6 & 0 & -5.039904 & 0.000000 & 1.206704 \\
\hline 40 & 1 & 0 & -3.099827 & 0.000000 & 2.146911 \\
\hline 41 & 6 & 0 & -5.741838 & 0.000000 & 0.000000 \\
\hline 42 & 1 & 0 & -5.578411 & 0.000000 & -2.150897 \\
\hline 43 & 1 & 0 & -5.578411 & 0.000000 & 2.150897 \\
\hline 44 & 1 & 0 & -6.828619 & 0.000000 & 0.000000 \\
\hline
\end{tabular}

diiodo-(9,10-diphenyl-1-anthracenyl)borane ( $\mathbf{P h}_{2}$-anthracene-1-BI $\left.\mathbf{I}_{2}\right)\left(C_{1}\right.$ symmetry)

$\mathrm{E}(\mathrm{B} 3 \mathrm{LYP} / 631 \mathrm{LAN})=-1048.69574007$ hartree

\begin{tabular}{cccccc}
$\begin{array}{c}\text { Center } \\
\text { Number }\end{array}$ & $\begin{array}{c}\text { Atomic } \\
\text { Number }\end{array}$ & $\begin{array}{c}\text { Atomic } \\
\text { Type }\end{array}$ & \multicolumn{3}{c}{ Coordinates (Angstroms) } \\
- \\
1 & 5 & 0 & 1.854154 & -1.003498 & Z \\
2 & 53 & 0 & 2.905850 & -0.660045 & 1.915564 \\
3 & 53 & 0 & 2.908332 & -0.676582 & -1.912232 \\
4 & 6 & 0 & -2.268250 & 4.137520 & -0.009643 \\
& & & &
\end{tabular}




$\begin{array}{rrrrrr}5 & 6 & 0 & -1.247183 & 3.225112 & -0.007416 \\ 6 & 6 & 0 & -1.504662 & 1.815387 & -0.004275 \\ 7 & 6 & 0 & -2.881232 & 1.370298 & -0.003414 \\ 8 & 6 & 0 & -3.915308 & 2.362423 & -0.005841 \\ 9 & 6 & 0 & -3.621704 & 3.699905 & -0.008864 \\ 10 & 6 & 0 & -0.457264 & 0.868763 & -0.001936 \\ 11 & 6 & 0 & -3.176148 & -0.010339 & -0.000191 \\ 12 & 6 & 0 & -2.128751 & -0.956964 & 0.002170 \\ 13 & 6 & 0 & -0.752180 & -0.511875 & 0.001265 \\ 14 & 6 & 0 & 0.281896 & -1.504001 & 0.003574 \\ 15 & 6 & 0 & -0.011708 & -2.841483 & 0.006642 \\ 16 & 6 & 0 & -1.365162 & -3.279097 & 0.007599 \\ 17 & 6 & 0 & -2.386229 & -2.366689 & 0.005422 \\ 18 & 1 & 0 & -2.046260 & 5.201392 & -0.011989 \\ 19 & 1 & 0 & -0.216893 & 3.563399 & -0.007991 \\ 20 & 1 & 0 & -4.948620 & 2.033484 & -0.005220 \\ 21 & 1 & 0 & -4.424526 & 4.432424 & -0.010651 \\ 22 & 1 & 0 & 0.791114 & -3.574002 & 0.008329 \\ 23 & 1 & 0 & -1.587151 & -4.342969 & 0.010041 \\ 24 & 1 & 0 & -3.416518 & -2.704977 & 0.006124 \\ 25 & 6 & 0 & -4.601970 & -0.471352 & 0.000720 \\ 26 & 6 & 0 & -5.283948 & -0.694349 & -1.205007 \\ 27 & 6 & 0 & -5.284013 & -0.689382 & 1.207319 \\ 28 & 6 & 0 & -6.612143 & -1.123799 & -1.204691 \\ 29 & 1 & 0 & -4.766138 & -0.528872 & -2.146082 \\ 30 & 6 & 0 & -6.612208 & -1.118827 & 1.208701 \\ 31 & 1 & 0 & -4.766253 & -0.520028 & 2.147732 \\ 32 & 6 & 0 & -7.280063 & -1.337262 & 0.002432 \\ 33 & 1 & 0 & -7.124509 & -1.291417 & -2.148553 \\ 34 & 1 & 0 & -7.124626 & -1.282552 & 2.153218 \\ 35 & 1 & 0 & -8.314135 & -1.671609 & 0.003093 \\ 36 & 6 & 0 & 0.968555 & 1.329782 & -0.002855 \\ 37 & 6 & 0 & 1.650825 & 1.551901 & 1.202868 \\ 38 & 6 & 0 & 1.650299 & 1.548711 & -1.209460 \\ 39 & 6 & 0 & 2.979015 & 1.981367 & 1.202544 \\ 40 & 1 & 0 & 1.133245 & 1.385731 & 2.143948 \\ 41 & 6 & 0 & 2.978494 & 1.978155 & -1.210851 \\ 42 & 1 & 0 & 1.132310 & 1.380046 & -2.149870 \\ 43 & 6 & 0 & 3.646641 & 2.195713 & -0.004585 \\ 44 & 1 & 0 & 3.491606 & 2.148308 & 2.146404 \\ 45 & 1 & 0 & 3.490684 & 2.142567 & -2.155373 \\ 46 & 1 & 0 & 4.680710 & 2.530067 & -0.005253\end{array}$

diiodo-(9,10-diphenyl-2-anthracenyl)borane ( $\mathbf{P h}_{2}$-anthracene-2-BI $\left.\mathbf{~}_{2}\right)\left(C_{1}\right.$ symmetry) $\mathrm{E}(\mathrm{B} 3 \mathrm{LYP} / 631 \mathrm{LAN})=-1048.71437996$ hartree

\begin{tabular}{cccccc}
$\begin{array}{c}\text { Center } \\
\text { Number }\end{array}$ & $\begin{array}{c}\text { Atomic } \\
\text { Number }\end{array}$ & $\begin{array}{c}\text { Atomic } \\
\text { Type }\end{array}$ & \multicolumn{2}{c}{ Coordinates (Angstroms) } \\
-1 & 5 & 0 & 2.761106 & -0.746042 & Z \\
\hline 2 & 53 & 0 & 3.866264 & -0.745038 & 1.914306 \\
3 & 53 & 0 & 3.865930 & -0.755959 & -1.913511 \\
4 & 6 & 0 & -5.245124 & 2.920787 & -0.001999 \\
5 & 6 & 0 & -3.875820 & 2.929481 & -0.001988 \\
6 & 6 & 0 & -3.120738 & 1.711499 & -0.001190 \\
7 & 6 & 0 & -3.842686 & 0.457768 & -0.000307 \\
8 & 6 & 0 & -5.275129 & 0.499449 & -0.000369 \\
9 & 6 & 0 & -5.954949 & 1.688109 & -0.001197 \\
& & 0 & & &
\end{tabular}




\begin{tabular}{rrrrrr}
10 & 6 & 0 & -1.708951 & 1.712493 & -0.001143 \\
11 & 6 & 0 & -3.134880 & -0.763770 & 0.000647 \\
12 & 6 & 0 & -1.723092 & -0.762776 & 0.000716 \\
13 & 6 & 0 & -1.001144 & 0.490955 & -0.000212 \\
14 & 6 & 0 & 0.431300 & 0.449273 & -0.000268 \\
15 & 1 & 0 & 0.976689 & 1.386548 & -0.001045 \\
16 & 6 & 0 & 1.111120 & -0.739387 & 0.000606 \\
17 & 6 & 0 & 0.401295 & -1.972065 & 0.001586 \\
18 & 6 & 0 & -0.968009 & -1.980759 & 0.001624 \\
19 & 1 & 0 & -5.794437 & 3.858531 & -0.002592 \\
20 & 1 & 0 & -3.338944 & 3.871659 & -0.002570 \\
21 & 1 & 0 & -5.820518 & -0.437827 & 0.000278 \\
22 & 1 & 0 & -7.041729 & 1.692493 & -0.001215 \\
23 & 1 & 0 & 0.950609 & -2.909808 & 0.002276 \\
24 & 1 & 0 & -1.504884 & -2.922937 & 0.002335 \\
25 & 6 & 0 & -3.882658 & -2.062357 & 0.001582 \\
26 & 6 & 0 & -4.240610 & -2.684220 & -1.204136 \\
27 & 6 & 0 & -4.240073 & -2.682793 & 1.208194 \\
28 & 6 & 0 & -4.937187 & -3.893894 & -1.203799 \\
29 & 1 & 0 & -3.969264 & -2.213198 & -2.145222 \\
30 & 6 & 0 & -4.936652 & -3.892464 & 1.209598 \\
31 & 1 & 0 & -3.968310 & -2.210656 & 2.148600 \\
32 & 6 & 0 & -5.287196 & -4.501467 & 0.003337 \\
33 & 1 & 0 & -5.206121 & -4.361121 & -2.147654 \\
34 & 1 & 0 & -5.205170 & -4.358572 & 2.154125 \\
35 & 1 & 0 & -5.829519 & -5.443261 & 0.004015 \\
36 & 6 & 0 & -0.961179 & 3.011083 & -0.002086 \\
37 & 6 & 0 & -0.602420 & 3.632490 & 1.203627 \\
38 & 6 & 0 & -0.604588 & 3.631985 & -1.208702 \\
39 & 6 & 0 & 0.094141 & 4.842172 & 1.203281 \\
40 & 1 & 0 & -0.873131 & 3.161109 & 2.144716 \\
41 & 6 & 0 & 0.091991 & 4.841655 & -1.210116 \\
42 & 1 & 0 & -0.876983 & 3.160205 & -2.149105 \\
43 & 6 & 0 & 0.443340 & 5.450203 & -0.003860 \\
44 & 1 & 0 & 0.363697 & 5.309049 & 2.147132 \\
45 & 1 & 0 & 0.359879 & 5.308119 & -2.154646 \\
46 & 1 & 0 & 0.985657 & 6.392002 & -0.004545 \\
------------------------------------------------------------------ \\
\hline 3
\end{tabular}

diiodo-(2-(10-phenylanthracen-9-yl)phenyl)borane ( $\mathbf{P h} \mathbf{h}_{2}$-anthracene-9a-BI $\left.\mathbf{I}_{2}\right)\left(C_{1}\right.$ symmetry) $\mathrm{E}(\mathrm{B} 3 \mathrm{LYP} / 631 \mathrm{LAN})=-1048.70302567$ hartree

\begin{tabular}{rrrrrr}
$\begin{array}{c}\text { Center } \\
\text { Number }\end{array}$ & $\begin{array}{c}\text { Atomic } \\
\text { Number }\end{array}$ & $\begin{array}{c}\text { Atomic } \\
\text { Type }\end{array}$ & \multicolumn{2}{c}{ Coordinates (Angstroms) } \\
- & 5 & 0 & X & $Z$ \\
2 & 53 & 0 & 2.256800 & 0.000493 & -0.471979 \\
3 & 53 & 0 & 2.087603 & 1.916962 & -1.559461 \\
4 & 6 & 0 & -0.661516 & -1.910859 & -1.568052 \\
5 & 6 & 0 & -0.016567 & -2.488468 & 1.218658 \\
6 & 6 & 0 & -0.648665 & -1.225597 & 1.226382 \\
7 & 6 & 0 & -1.997723 & -1.224042 & 0.703804 \\
8 & 6 & 0 & -2.631402 & -2.485454 & 0.456981 \\
9 & 6 & 0 & -1.987946 & -3.668485 & 0.704917 \\
10 & 6 & 0 & 0.009868 & -0.003410 & 1.482773 \\
11 & 6 & 0 & -2.654662 & -0.000337 & 0.450580 \\
12 & 6 & 0 & -1.996122 & 1.221852 & 0.706951 \\
13 & 6 & 0 & -0.647078 & 1.220296 & 1.229571 \\
14 & 6 & 0 & -0.013443 & 2.481709 & 1.476504
\end{tabular}




$\begin{array}{llrrrr}15 & 1 & 0 & 0.997698 & 2.485457 & 1.868315 \\ 16 & 6 & 0 & -0.656882 & 3.664740 & 1.228525 \\ 17 & 6 & 0 & -1.983247 & 3.666269 & 0.714619 \\ 18 & 6 & 0 & -2.628179 & 2.484724 & 0.463454 \\ 19 & 1 & 0 & -0.159922 & -4.614572 & 1.411843 \\ 20 & 1 & 0 & 0.994639 & -2.494545 & 1.861389 \\ 21 & 1 & 0 & -3.642590 & -2.489201 & 0.065291 \\ 22 & 1 & 0 & -2.490711 & -4.611886 & 0.509137 \\ 23 & 1 & 0 & -0.154153 & 4.608141 & 1.424398 \\ 24 & 1 & 0 & -2.484806 & 4.610828 & 0.521343 \\ 25 & 1 & 0 & -3.639339 & 2.490802 & 0.071722 \\ 26 & 6 & 0 & -4.051981 & 0.001274 & -0.090713 \\ 27 & 6 & 0 & -5.156054 & 0.000501 & 0.775093 \\ 28 & 6 & 0 & -4.284660 & 0.003586 & -1.474352 \\ 29 & 6 & 0 & -6.457891 & 0.002002 & 0.271360 \\ 30 & 1 & 0 & -4.988441 & -0.001286 & 1.848888 \\ 31 & 6 & 0 & -5.586111 & 0.005085 & -1.979080 \\ 32 & 1 & 0 & -3.437405 & 0.004203 & -2.155006 \\ 33 & 6 & 0 & -6.676537 & 0.004298 & -1.107414 \\ 34 & 1 & 0 & -7.301105 & 0.001375 & 0.957275 \\ 35 & 1 & 0 & -5.747195 & 0.006869 & -3.054040 \\ 36 & 1 & 0 & -7.689937 & 0.005466 & -1.499985 \\ 37 & 6 & 0 & 1.407184 & -0.005027 & 2.024074 \\ 38 & 6 & 0 & 2.511262 & -0.003328 & 1.158277 \\ 39 & 6 & 0 & 1.639854 & -0.008286 & 3.407712 \\ 40 & 6 & 0 & 3.813096 & -0.004846 & 1.662019 \\ 41 & 6 & 0 & 2.941301 & -0.009785 & 3.912449 \\ 42 & 1 & 0 & 0.792594 & -0.009629 & 4.088360 \\ 43 & 6 & 0 & 4.031733 & -0.008073 & 3.040792 \\ 44 & 1 & 0 & 4.656314 & -0.003506 & 0.976110 \\ 45 & 1 & 0 & 3.102377 & -0.012292 & 4.987409 \\ 46 & 1 & 0 & 5.045131 & -0.009249 & 3.433370 \\ ----------------------------------------------------------------------------------------------\end{array}$

diiodo-(3-(10-phenylanthracen-9-yl)phenyl)borane ( $\mathbf{P h}_{2}$-anthracene-9b-BI $)\left(C_{1}\right.$ symmetry) $\mathrm{E}(\mathrm{B} 3 \mathrm{LYP} / 631 \mathrm{LAN})=-1048.71166931$ hartree

\begin{tabular}{rrrrrr} 
Center & Atomic & Atomic & \multicolumn{2}{c}{ Coordinates (Angstroms) } \\
Number & Number & Type & X & Y & $Z$ \\
-1 & 5 & 0 & 3.609471 & -0.000250 & -0.006614 \\
2 & 53 & 0 & 4.456292 & 1.914131 & -0.715230 \\
3 & 53 & 0 & 4.455141 & -1.913699 & -0.719115 \\
4 & 6 & 0 & -2.134273 & -3.667731 & 0.686430 \\
5 & 6 & 0 & -1.485767 & -2.485665 & 0.925653 \\
6 & 6 & 0 & -2.121873 & -1.223304 & 0.690190 \\
7 & 6 & 0 & -3.478908 & -1.222837 & 0.188686 \\
8 & 6 & 0 & -4.116060 & -2.484761 & -0.046296 \\
9 & 6 & 0 & -3.468544 & -3.667273 & 0.193410 \\
10 & 6 & 0 & -1.459713 & -0.000584 & 0.934411 \\
11 & 6 & 0 & -4.139997 & 0.000339 & -0.056159 \\
12 & 6 & 0 & -3.477828 & 1.223059 & 0.188042 \\
13 & 6 & 0 & -2.120809 & 1.222592 & 0.689588 \\
14 & 6 & 0 & -1.483698 & 2.484517 & 0.924680 \\
15 & 1 & 0 & -0.466579 & 2.489081 & 1.300688 \\
16 & 6 & 0 & -2.131198 & 3.667029 & 0.684932 \\
17 & 6 & 0 & -3.465407 & 3.667488 & 0.191745 \\
18 & 6 & 0 & -4.113896 & 2.485421 & -0.047525 \\
19 & 1 & 0 & -1.629510 & -4.611886 & 0.873233 \\
& & 0 & &
\end{tabular}




$\begin{array}{rrrrrr}20 & 1 & 0 & -0.468583 & -2.490926 & 1.301478 \\ 21 & 1 & 0 & -5.133224 & -2.489324 & -0.422182 \\ 22 & 1 & 0 & -3.974077 & -4.611081 & 0.006937 \\ 23 & 1 & 0 & -1.625700 & 4.610837 & 0.871499 \\ 24 & 1 & 0 & -3.970136 & 4.611642 & 0.004851 \\ 25 & 1 & 0 & -5.131036 & 2.490683 & -0.423469 \\ 26 & 6 & 0 & -5.545577 & 0.000821 & -0.575625 \\ 27 & 6 & 0 & -6.636031 & 0.001152 & 0.307273 \\ 28 & 6 & 0 & -5.799779 & 0.000950 & -1.955473 \\ 29 & 6 & 0 & -7.945556 & 0.001603 & -0.176124 \\ 30 & 1 & 0 & -6.451714 & 0.001057 & 1.378329 \\ 31 & 6 & 0 & -7.108934 & 0.001398 & -2.439871 \\ 32 & 1 & 0 & -4.963228 & 0.000694 & -2.649242 \\ 33 & 6 & 0 & -8.185651 & 0.001727 & -1.551327 \\ 34 & 1 & 0 & -8.777984 & 0.001858 & 0.522841 \\ 35 & 1 & 0 & -7.286741 & 0.001489 & -3.512194 \\ 36 & 1 & 0 & -9.205042 & 0.002076 & -1.928068 \\ 37 & 6 & 0 & -0.054135 & -0.001073 & 1.453885 \\ 38 & 6 & 0 & 1.036324 & -0.000478 & 0.570994 \\ 39 & 6 & 0 & 0.200057 & -0.002149 & 2.833734 \\ 40 & 6 & 0 & 2.345846 & -0.000947 & 1.054400 \\ 41 & 1 & 0 & 0.852014 & 0.000346 & -0.500062 \\ 42 & 6 & 0 & 1.509209 & -0.002596 & 3.318141 \\ 43 & 1 & 0 & -0.636498 & -0.002619 & 3.527497 \\ 44 & 6 & 0 & 2.585932 & -0.002001 & 2.429604 \\ 45 & 1 & 0 & 1.687009 & -0.003411 & 4.390464 \\ 46 & 1 & 0 & 3.605321 & -0.002358 & 2.806351 \\ --------------------------------------\end{array}$

diiodo-(4-(10-phenylanthracen-9-yl)phenyl)borane ( $\mathbf{P h}_{2}$-anthracene-9c-BI $)\left(C_{1}\right.$ symmetry) $\mathrm{E}(\mathrm{B} 3 \mathrm{LYP} / 631 \mathrm{LAN})=-1048.71258701$ hartree

\begin{tabular}{|c|c|c|c|c|c|}
\hline \multirow{2}{*}{$\begin{array}{l}\text { Center } \\
\text { Number }\end{array}$} & \multirow{2}{*}{$\begin{array}{l}\text { Atomic } \\
\text { Number }\end{array}$} & \multirow{2}{*}{$\begin{array}{l}\text { Atomic } \\
\text { Type }\end{array}$} & \multicolumn{3}{|c|}{ Coordinates (Angstroms) } \\
\hline & & & $\mathrm{X}$ & $\mathrm{Y}$ & $\mathrm{Z}$ \\
\hline 1 & 5 & 0 & 4.114389 & 0.000000 & 0.000007 \\
\hline 2 & 53 & 0 & 5.219394 & 1.913913 & 0.001576 \\
\hline 3 & 53 & 0 & 5.219385 & -1.913918 & -0.001572 \\
\hline 4 & 6 & 0 & -2.566211 & -3.667370 & 0.001738 \\
\hline 5 & 6 & 0 & -1.875375 & -2.485078 & 0.001152 \\
\hline 6 & 6 & 0 & -2.554075 & -1.222938 & 0.000629 \\
\hline 7 & 6 & 0 & -4.000813 & -1.222944 & 0.000653 \\
\hline 8 & 6 & 0 & -4.679502 & -2.485090 & 0.001293 \\
\hline 9 & 6 & 0 & -3.988655 & -3.667376 & 0.001829 \\
\hline 10 & 6 & 0 & -1.848712 & 0.000011 & -0.000008 \\
\hline 11 & 6 & 0 & -4.706185 & 0.000001 & -0.000004 \\
\hline 12 & 6 & 0 & -4.000822 & 1.222952 & -0.000663 \\
\hline 13 & 6 & 0 & -2.554084 & 1.222957 & -0.000643 \\
\hline 14 & 6 & 0 & -1.875396 & 2.485104 & -0.001164 \\
\hline 15 & 1 & 0 & -0.791002 & 2.490022 & -0.001068 \\
\hline 16 & 6 & 0 & -2.566242 & 3.667390 & -0.001746 \\
\hline 17 & 6 & 0 & -3.988686 & 3.667384 & -0.001833 \\
\hline 18 & 6 & 0 & -4.679522 & 2.485092 & -0.001297 \\
\hline 19 & 1 & 0 & -2.027682 & -4.611349 & 0.002107 \\
\hline 20 & 1 & 0 & -0.790980 & -2.489985 & 0.001054 \\
\hline 21 & 1 & 0 & -5.763896 & -2.490007 & 0.001327 \\
\hline 22 & 1 & 0 & -4.527174 & -4.611360 & 0.002298 \\
\hline 23 & 1 & 0 & -2.027722 & 4.611374 & -0.002115 \\
\hline \multirow[t]{2}{*}{24} & 1 & 0 & -4.527214 & 4.611363 & -0.002299 \\
\hline & & & $\mathrm{S} 40$ & & \\
\hline
\end{tabular}




\begin{tabular}{rrrrrr}
25 & 1 & 0 & -5.763916 & 2.490000 & -0.001327 \\
26 & 6 & 0 & -6.204685 & -0.000006 & 0.000001 \\
27 & 6 & 0 & -6.921454 & 0.000258 & 1.206169 \\
28 & 6 & 0 & -6.921463 & -0.000278 & -1.206161 \\
29 & 6 & 0 & -8.317351 & 0.000253 & 1.206708 \\
30 & 1 & 0 & -6.377273 & 0.000470 & 2.146913 \\
31 & 6 & 0 & -8.317360 & -0.000286 & -1.206690 \\
32 & 1 & 0 & -6.377289 & -0.000486 & -2.146909 \\
33 & 6 & 0 & -9.019287 & -0.000020 & 0.000012 \\
34 & 1 & 0 & -8.855857 & 0.000462 & 2.150901 \\
35 & 1 & 0 & -8.855873 & -0.000502 & -2.150879 \\
36 & 1 & 0 & -10.106068 & -0.000025 & 0.000016 \\
37 & 6 & 0 & -0.350213 & 0.000012 & -0.000005 \\
38 & 6 & 0 & 0.366564 & 0.000674 & -1.206168 \\
39 & 6 & 0 & 0.366558 & -0.000663 & 1.206162 \\
40 & 6 & 0 & 1.762461 & 0.000662 & -1.206698 \\
41 & 1 & 0 & -0.177611 & 0.001191 & -2.146916 \\
42 & 6 & 0 & 1.762455 & -0.000654 & 1.206699 \\
43 & 1 & 0 & -0.177622 & -0.001181 & 2.146906 \\
44 & 6 & 0 & 2.464389 & 0.000004 & 0.000002 \\
45 & 1 & 0 & 2.300973 & 0.001166 & -2.150888 \\
46 & 1 & 0 & 2.300962 & -0.001163 & 2.150892 \\
\hline--------------------------------------------------------------------------------------------------------
\end{tabular}

2-(9,10-diphenyl-2-anthracenyl)-4,4,5,5-tetramethyl-1,3,2-dioxaborolane

$\left(\mathbf{P h}_{2}\right.$-anthracene-2-

Bpin) ( $C_{1}$ symmetry)

$\mathrm{E}(\mathrm{B} 3 \mathrm{LYP} / 6-31 \mathrm{G}(\mathrm{d}))=-1412.32338821$ hartree

\begin{tabular}{|c|c|c|c|c|c|}
\hline \multirow{2}{*}{$\begin{array}{l}\text { Center } \\
\text { Number }\end{array}$} & \multirow{2}{*}{$\begin{array}{l}\text { Atomic } \\
\text { Number }\end{array}$} & \multirow{2}{*}{$\begin{array}{l}\text { Atomic } \\
\text { Type }\end{array}$} & \multicolumn{3}{|c|}{ Coordinates (Angstroms) } \\
\hline & & & $X$ & $\mathrm{Y}$ & Z \\
\hline 1 & 5 & 0 & -3.169623 & 0.807275 & -0.000096 \\
\hline 2 & 8 & 0 & -3.922392 & 0.320767 & -1.230014 \\
\hline 3 & 8 & 0 & -3.921197 & 0.323313 & 1.231920 \\
\hline 4 & 6 & 0 & 5.514128 & 1.428027 & 0.000694 \\
\hline 5 & 6 & 0 & 4.237307 & 1.922797 & 0.001142 \\
\hline 6 & 6 & 0 & 3.098655 & 1.052681 & 0.000286 \\
\hline 7 & 6 & 0 & 3.327902 & -0.375778 & -0.001156 \\
\hline 8 & 6 & 0 & 4.681643 & -0.845900 & -0.001560 \\
\hline 9 & 6 & 0 & 5.739526 & 0.023556 & -0.000657 \\
\hline 10 & 6 & 0 & 1.779388 & 1.555352 & 0.000709 \\
\hline 11 & 6 & 0 & 2.232176 & -1.266018 & -0.002184 \\
\hline 12 & 6 & 0 & 0.912908 & -0.763348 & -0.001783 \\
\hline 13 & 6 & 0 & 0.683661 & 0.665111 & -0.000296 \\
\hline 14 & 6 & 0 & -0.670081 & 1.135233 & 0.000226 \\
\hline 15 & 1 & 0 & -0.846763 & 2.205147 & 0.001422 \\
\hline 16 & 6 & 0 & -1.727964 & 0.265778 & -0.000724 \\
\hline 17 & 6 & 0 & -1.502566 & -1.138694 & -0.002252 \\
\hline 18 & 6 & 0 & -0.225745 & -1.633463 & -0.002749 \\
\hline 19 & 1 & 0 & 6.360849 & 2.109329 & 0.001343 \\
\hline 20 & 1 & 0 & 4.070326 & 2.994269 & 0.002142 \\
\hline 21 & 1 & 0 & 4.858325 & -1.915815 & -0.002627 \\
\hline 22 & 1 & 0 & 6.756914 & -0.358583 & -0.000999 \\
\hline 23 & 1 & 0 & -2.349288 & -1.819995 & -0.002998 \\
\hline 24 & 1 & 0 & -0.058765 & -2.704935 & -0.003877 \\
\hline 25 & 6 & 0 & 2.469625 & -2.745585 & -0.003697 \\
\hline 26 & 6 & 0 & 2.583469 & -3.454493 & 1.201743 \\
\hline \multirow[t]{2}{*}{27} & 6 & 0 & 2.582941 & -3.452111 & -1.210586 \\
\hline & & & S41 & & \\
\hline
\end{tabular}




\begin{tabular}{|c|c|c|c|c|c|}
\hline 28 & 6 & 0 & 2.804659 & -4.832754 & 1.200867 \\
\hline 29 & 1 & 0 & 2.497444 & -2.918121 & 2.143039 \\
\hline 30 & 6 & 0 & 2.804134 & -4.830370 & -1.212529 \\
\hline 31 & 1 & 0 & 2.496507 & -2.913879 & -2.150782 \\
\hline 32 & 6 & 0 & 2.915623 & -5.524625 & -0.006540 \\
\hline 33 & 1 & 0 & 2.890195 & -5.365393 & 2.144514 \\
\hline 34 & 1 & 0 & 2.889261 & -5.361142 & -2.157264 \\
\hline 35 & 1 & 0 & 3.087833 & -6.597674 & -0.007637 \\
\hline 36 & 6 & 0 & 1.541945 & 3.034919 & 0.002230 \\
\hline 37 & 6 & 0 & 1.427186 & 3.743688 & -1.203205 \\
\hline 38 & 6 & 0 & 1.429565 & 3.741587 & 1.209123 \\
\hline 39 & 6 & 0 & 1.206013 & 5.121952 & -1.202320 \\
\hline 40 & 1 & 0 & 1.512489 & 3.207206 & -2.144503 \\
\hline 41 & 6 & 0 & 1.208372 & 5.119846 & 1.211076 \\
\hline 42 & 1 & 0 & 1.516717 & 3.203465 & 2.149315 \\
\hline 43 & 6 & 0 & 1.095969 & 5.813962 & 0.005091 \\
\hline 44 & 1 & 0 & 1.119771 & 5.654483 & -2.145963 \\
\hline 45 & 1 & 0 & 1.123960 & 5.650727 & 2.155814 \\
\hline 46 & 1 & 0 & 0.923767 & 6.887013 & 0.006196 \\
\hline 47 & 6 & 0 & -5.191445 & -0.374313 & -0.758389 \\
\hline 48 & 6 & 0 & -5.155858 & -0.432617 & 0.762055 \\
\hline 49 & 6 & 0 & -6.431446 & 0.193287 & 1.355957 \\
\hline 50 & 1 & 0 & -7.285723 & -0.364977 & 1.034320 \\
\hline 51 & 1 & 0 & -6.374207 & 0.173824 & 2.424248 \\
\hline 52 & 1 & 0 & -6.520691 & 1.205895 & 1.021950 \\
\hline 53 & 6 & 0 & -6.434383 & 0.399926 & -1.235106 \\
\hline 54 & 1 & 0 & -6.423658 & 0.469160 & -2.302810 \\
\hline 55 & 1 & 0 & -7.317859 & -0.114888 & -0.919929 \\
\hline 56 & 1 & 0 & -6.425231 & 1.383451 & -0.813805 \\
\hline 57 & 6 & 0 & -5.285640 & -1.791870 & -1.352745 \\
\hline 58 & 1 & 0 & -6.312729 & -2.085649 & -1.413435 \\
\hline 59 & 1 & 0 & -4.854631 & -1.797366 & -2.332082 \\
\hline 60 & 1 & 0 & -4.755008 & -2.477522 & -0.725679 \\
\hline 61 & 6 & 0 & -5.103278 & -1.895057 & 1.241745 \\
\hline 62 & 1 & 0 & -4.908943 & -1.918608 & 2.293686 \\
\hline 63 & 1 & 0 & -6.040952 & -2.370018 & 1.041547 \\
\hline 64 & 1 & 0 & -4.323407 & -2.412656 & 0.723294 \\
\hline
\end{tabular}

phenanthrene (1f) ( $C_{2}$ symmetry)

$\mathrm{E}(\mathrm{B} 3 \mathrm{LYP} / 6-31 \mathrm{G}(\mathrm{d}))=-539.53865624$ hartree

\begin{tabular}{|c|c|c|c|c|c|}
\hline \multirow{2}{*}{$\begin{array}{l}\text { Center } \\
\text { Number }\end{array}$} & \multirow{2}{*}{$\begin{array}{l}\text { Atomic } \\
\text { Number }\end{array}$} & \multirow{2}{*}{$\begin{array}{l}\text { Atomic } \\
\text { Type }\end{array}$} & \multicolumn{3}{|c|}{ Coordinates (Angstroms) } \\
\hline & & & $\mathrm{X}$ & $\mathrm{Y}$ & Z \\
\hline 1 & 6 & 0 & 3.561758 & -0.296277 & 0.000042 \\
\hline 2 & 6 & 0 & 2.837358 & 0.879092 & 0.000123 \\
\hline 3 & 6 & 0 & 1.422926 & 0.865926 & 0.000067 \\
\hline 4 & 6 & 0 & 0.728916 & -0.380865 & -0.000018 \\
\hline 5 & 6 & 0 & 1.500367 & -1.566900 & -0.000149 \\
\hline 6 & 6 & 0 & 2.882905 & -1.529303 & -0.000119 \\
\hline 7 & 6 & 0 & 0.679767 & 2.093896 & 0.000049 \\
\hline 8 & 6 & 0 & -0.728917 & -0.380863 & 0.000007 \\
\hline 9 & 6 & 0 & -1.422925 & 0.865925 & -0.000073 \\
\hline 10 & 6 & 0 & -0.679768 & 2.093896 & -0.000065 \\
\hline 11 & 6 & 0 & -2.837360 & 0.879092 & -0.000104 \\
\hline 12 & 1 & 0 & -3.347926 & 1.839431 & -0.000184 \\
\hline 13 & 6 & 0 & -3.561757 & -0.296276 & -0.000022 \\
\hline 14 & 6 & 0 & -2.882903 & -1.529305 & 0.000121 \\
\hline & & & $\mathrm{S} 42$ & & \\
\hline
\end{tabular}




$\begin{array}{rrrrrr}15 & 6 & 0 & -1.500368 & -1.566902 & 0.000138 \\ 16 & 1 & 0 & 1.232810 & 3.030187 & 0.000111 \\ 17 & 1 & 0 & 4.648026 & -0.271894 & 0.000087 \\ 18 & 1 & 0 & 3.347926 & 1.839431 & 0.000214 \\ 19 & 1 & 0 & 1.006893 & -2.532907 & -0.000329 \\ 20 & 1 & 0 & 3.446835 & -2.458226 & -0.000243 \\ 21 & 1 & 0 & -1.232809 & 3.030187 & -0.000135 \\ 22 & 1 & 0 & -4.648025 & -0.271899 & -0.000043 \\ 23 & 1 & 0 & -3.446840 & -2.458223 & 0.000238 \\ 24 & 1 & 0 & -1.006884 & -2.532904 & 0.000303\end{array}$

diiodo-(phenanthren-1-yl)borane (phenanthrene-1-BI $\left.\mathbf{B}_{2}\right)\left(C_{1}\right.$ symmetry)

$\mathrm{E}(\mathrm{B} 3 \mathrm{LYP} / 631 \mathrm{LAN})=-586.61027263$ hartree

\begin{tabular}{|c|c|c|c|c|c|}
\hline \multirow{2}{*}{$\begin{array}{l}\text { Center } \\
\text { Number }\end{array}$} & \multirow{2}{*}{$\begin{array}{l}\text { Atomic } \\
\text { Number }\end{array}$} & \multirow{2}{*}{$\begin{array}{l}\text { Atomic } \\
\text { Type }\end{array}$} & \multicolumn{3}{|c|}{ Coordinates (Angstroms) } \\
\hline & & & $\mathrm{X}$ & $\mathrm{Y}$ & Z \\
\hline 1 & 5 & 0 & -1.476427 & -0.000266 & 0.271374 \\
\hline 2 & 53 & 0 & -2.441404 & 1.914102 & -0.265390 \\
\hline 3 & 53 & 0 & -2.441639 & -1.913729 & -0.268187 \\
\hline 4 & 6 & 0 & 5.968930 & 0.001583 & -1.362671 \\
\hline 5 & 6 & 0 & 4.730748 & 0.003742 & -1.971770 \\
\hline 6 & 6 & 0 & 3.548242 & 0.001666 & -1.197599 \\
\hline 7 & 6 & 0 & 3.630416 & -0.000598 & 0.215711 \\
\hline 8 & 6 & 0 & 4.912372 & -0.002323 & 0.813064 \\
\hline 9 & 6 & 0 & 6.057401 & -0.001729 & 0.040596 \\
\hline 10 & 6 & 0 & 2.264309 & 0.002253 & -1.838664 \\
\hline 11 & 6 & 0 & 2.412057 & -0.001209 & 0.994587 \\
\hline 12 & 6 & 0 & 1.163819 & -0.000685 & 0.326735 \\
\hline 13 & 6 & 0 & 1.120899 & 0.000611 & -1.107700 \\
\hline 14 & 6 & 0 & -0.035337 & -0.000942 & 1.074964 \\
\hline 15 & 6 & 0 & -0.002232 & -0.001479 & 2.454431 \\
\hline 16 & 6 & 0 & 1.234295 & -0.001899 & 3.123800 \\
\hline 17 & 6 & 0 & 2.416085 & -0.001921 & 2.408913 \\
\hline 18 & 1 & 0 & 2.237773 & 0.003794 & -2.939164 \\
\hline 19 & 1 & 0 & 6.888702 & 0.002346 & -1.965493 \\
\hline 20 & 1 & 0 & 4.647573 & 0.006406 & -3.069117 \\
\hline 21 & 1 & 0 & 5.007921 & -0.004386 & 1.908736 \\
\hline 22 & 1 & 0 & 7.047576 & -0.003709 & 0.519978 \\
\hline 23 & 1 & 0 & 0.133343 & 0.000706 & -1.594049 \\
\hline 24 & 1 & 0 & -0.935477 & -0.001561 & 3.036193 \\
\hline 25 & 1 & 0 & 1.253459 & -0.002280 & 4.223747 \\
\hline 26 & 1 & 0 & 3.370515 & -0.002183 & 2.955445 \\
\hline
\end{tabular}

diiodo-(phenanthren-2-yl)borane (phenanthrene-2-BI 2$)\left(C_{1}\right.$ symmetry)

$\mathrm{E}(\mathrm{B} 3 \mathrm{LYP} / 631 \mathrm{LAN})=-586.61234556$ hartree

\begin{tabular}{cccccc} 
Center & Atomic & Atomic & \multicolumn{2}{c}{ Coordinates (Angstroms) } \\
Number & Number & Type & X & Y & Z \\
-1 & 5 & 0 & 1.820015 & -0.000038 & -0.069226 \\
2 & 53 & 0 & 2.923317 & 1.914134 & -0.016571 \\
3 & 53 & 0 & 2.924119 & -1.913698 & -0.014838 \\
4 & 6 & 0 & -6.910135 & 0.000468 & -0.412007 \\
5 & 6 & 0 & -6.239228 & 0.002782 & 0.793803 \\
6 & 6 & 0 & -4.826326 & 0.001141 & 0.830861 \\
& & & &
\end{tabular}




$\begin{array}{rrrrrr}7 & 6 & 0 & -4.089007 & -0.000844 & -0.377675 \\ 8 & 6 & 0 & -4.802586 & -0.002736 & -1.598759 \\ 9 & 6 & 0 & -6.183711 & -0.002569 & -1.615874 \\ 10 & 6 & 0 & -4.136015 & 0.001887 & 2.089002 \\ 11 & 6 & 0 & -2.643956 & -0.001011 & -0.324067 \\ 12 & 6 & 0 & -1.998241 & -0.000342 & 0.935765 \\ 13 & 6 & 0 & -2.779857 & 0.000662 & 2.139311 \\ 14 & 6 & 0 & -0.586428 & -0.000165 & 1.003628 \\ 15 & 1 & 0 & -0.101585 & 0.000393 & 1.991563 \\ 16 & 6 & 0 & 0.171952 & -0.000419 & -0.149145 \\ 17 & 6 & 0 & -0.463273 & -0.000982 & -1.403555 \\ 18 & 6 & 0 & -1.841823 & -0.001425 & -1.488935 \\ 19 & 1 & 0 & -4.740926 & 0.003202 & 3.008723 \\ 20 & 1 & 0 & -8.009487 & 0.000893 & -0.440288 \\ 21 & 1 & 0 & -6.795786 & 0.005233 & 1.743188 \\ 22 & 1 & 0 & -4.257154 & -0.004590 & -2.553814 \\ 23 & 1 & 0 & -6.724630 & -0.004675 & -2.573819 \\ 24 & 1 & 0 & -2.245058 & 0.000880 & 3.101491 \\ 25 & 1 & 0 & 0.147383 & -0.001135 & -2.318623 \\ 26 & 1 & 0 & -2.315119 & -0.001791 & -2.481722\end{array}$

diiodo-(phenanthren-3-yl)borane (phenanthrene-3-BI $)\left(C_{1}\right.$ symmetry)

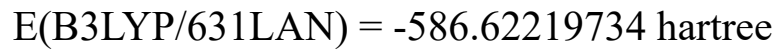

\begin{tabular}{|c|c|c|c|c|c|}
\hline \multirow{2}{*}{$\begin{array}{l}\text { Center } \\
\text { Number }\end{array}$} & \multirow{2}{*}{$\begin{array}{l}\text { Atomic } \\
\text { Number }\end{array}$} & \multirow{2}{*}{$\begin{array}{l}\text { Atomic } \\
\text { Type }\end{array}$} & \multicolumn{3}{|c|}{ Coordinates (Angstroms) } \\
\hline & & & $X$ & $\mathrm{Y}$ & $\mathrm{Z}$ \\
\hline 1 & 5 & 0 & 1.649466 & 0.000093 & 0.154724 \\
\hline 2 & 53 & 0 & 2.675565 & 1.914186 & -0.254508 \\
\hline 3 & 53 & 0 & 2.676659 & -1.913646 & -0.253416 \\
\hline 4 & 6 & 0 & -5.754365 & -0.000143 & -2.077298 \\
\hline 5 & 6 & 0 & -5.886279 & 0.002010 & -0.703729 \\
\hline 6 & 6 & 0 & -4.743977 & 0.000619 & 0.128624 \\
\hline 7 & 6 & 0 & -3.451033 & -0.000943 & -0.448003 \\
\hline 8 & 6 & 0 & -3.345586 & -0.002678 & -1.858365 \\
\hline 9 & 6 & 0 & -4.473042 & -0.002760 & -2.656264 \\
\hline 10 & 6 & 0 & -4.889612 & 0.001195 & 1.556293 \\
\hline 11 & 6 & 0 & -2.291654 & -0.000857 & 0.416222 \\
\hline 12 & 6 & 0 & -2.474967 & -0.000368 & 1.819975 \\
\hline 13 & 6 & 0 & -3.801551 & 0.000207 & 2.367354 \\
\hline 14 & 6 & 0 & -1.351044 & 0.000053 & 2.677074 \\
\hline 15 & 1 & 0 & -1.512509 & 0.000466 & 3.765659 \\
\hline 16 & 6 & 0 & -0.072408 & 0.000211 & 2.158313 \\
\hline 17 & 6 & 0 & 0.116466 & -0.000171 & 0.764979 \\
\hline 18 & 6 & 0 & -0.970128 & -0.000848 & -0.087667 \\
\hline 19 & 1 & 0 & -5.909628 & 0.002180 & 1.970263 \\
\hline 20 & 1 & 0 & -6.643483 & 0.000087 & -2.724482 \\
\hline 21 & 1 & 0 & -6.883318 & 0.004133 & -0.237893 \\
\hline 22 & 1 & 0 & -2.354490 & -0.004205 & -2.335183 \\
\hline 23 & 1 & 0 & -4.374768 & -0.004739 & -3.751981 \\
\hline 24 & 1 & 0 & -3.907267 & 0.000296 & 3.463084 \\
\hline 25 & 1 & 0 & 0.801774 & 0.000655 & 2.825547 \\
\hline 26 & 1 & 0 & -0.796403 & -0.001066 & -1.173693 \\
\hline
\end{tabular}

diiodo-(phenanthren-4-yl)borane (phenanthrene-4-BI $)\left(C_{1}\right.$ symmetry)

$\mathrm{E}(\mathrm{B} 3 \mathrm{LYP} / 631 \mathrm{LAN})=-586.60662477$ hartree 


\begin{tabular}{|c|c|c|c|c|c|}
\hline \multirow{2}{*}{$\begin{array}{l}\text { Center } \\
\text { Number }\end{array}$} & \multirow{2}{*}{$\begin{array}{l}\text { Atomic } \\
\text { Number }\end{array}$} & \multirow{2}{*}{$\begin{array}{l}\text { Atomic } \\
\text { Type }\end{array}$} & \multicolumn{3}{|c|}{ Coordinates (Angstroms) } \\
\hline & & & $X$ & $\mathrm{Y}$ & Z \\
\hline 1 & 5 & 0 & -0.933326 & 0.516845 & 0.000960 \\
\hline 2 & 53 & 0 & -1.861804 & -0.083517 & 1.914490 \\
\hline 3 & 53 & 0 & -1.864544 & -0.076792 & -1.913336 \\
\hline 4 & 6 & 0 & 2.159938 & -3.484678 & -0.005024 \\
\hline 5 & 6 & 0 & 3.271964 & -2.667677 & -0.002545 \\
\hline 6 & 6 & 0 & 3.131317 & -1.261304 & -0.002070 \\
\hline 7 & 6 & 0 & 1.839764 & -0.681568 & -0.002081 \\
\hline 8 & 6 & 0 & 0.717943 & -1.542822 & -0.004192 \\
\hline 9 & 6 & 0 & 0.874384 & -2.915164 & -0.006107 \\
\hline 10 & 6 & 0 & 4.292823 & -0.418479 & -0.001166 \\
\hline 11 & 6 & 0 & 1.711501 & 0.758775 & -0.000094 \\
\hline 12 & 6 & 0 & 2.880284 & 1.557570 & 0.000663 \\
\hline 13 & 6 & 0 & 4.172448 & 0.933263 & -0.000370 \\
\hline 14 & 6 & 0 & 2.770337 & 2.966728 & 0.002933 \\
\hline 15 & 1 & 0 & 3.689574 & 3.571783 & 0.003528 \\
\hline 16 & 6 & 0 & 1.531463 & 3.574357 & 0.004645 \\
\hline 17 & 6 & 0 & 0.366742 & 2.786651 & 0.004006 \\
\hline 18 & 6 & 0 & 0.455134 & 1.408292 & 0.001532 \\
\hline 19 & 1 & 0 & 5.281221 & -0.903119 & -0.001420 \\
\hline 20 & 1 & 0 & 2.269920 & -4.578880 & -0.006247 \\
\hline 21 & 1 & 0 & 4.283721 & -3.100623 & -0.001610 \\
\hline 22 & 1 & 0 & -0.298041 & -1.121626 & -0.004543 \\
\hline 23 & 1 & 0 & -0.008061 & -3.572083 & -0.008356 \\
\hline 24 & 1 & 0 & 5.059861 & 1.584644 & -0.000021 \\
\hline 25 & 1 & 0 & 1.446384 & 4.670784 & 0.006528 \\
\hline 26 & 1 & 0 & -0.617760 & 3.277573 & 0.005426 \\
\hline
\end{tabular}

diiodo-(phenanthren-9-yl)borane (phenanthrene-9-BI 2$)\left(C_{1}\right.$ symmetry)

$\mathrm{E}(\mathrm{B} 3 \mathrm{LYP} / 631 \mathrm{LAN})=-586.61217458$ hartree

\begin{tabular}{rrrrrr} 
Center & Atomic & Atomic & \multicolumn{2}{c}{ Coordinates (Angstroms) } \\
Number & Number & Type & X & Z \\
-1 & 5 & 0 & -1.167246 & -0.251849 & -0.000068 \\
2 & 53 & 0 & -2.272446 & -0.268771 & -1.913794 \\
3 & 53 & 0 & -2.271745 & -0.271438 & 1.914037 \\
4 & 6 & 0 & 4.757687 & -2.524900 & -0.002204 \\
5 & 6 & 0 & 3.377976 & -2.547091 & -0.004008 \\
6 & 6 & 0 & 2.639164 & -1.342176 & -0.001854 \\
7 & 6 & 0 & 3.316850 & -0.099220 & 0.000121 \\
8 & 6 & 0 & 4.731132 & -0.106340 & 0.001484 \\
9 & 6 & 0 & 5.436781 & -1.293714 & 0.000821 \\
10 & 6 & 0 & 1.204433 & -1.373740 & -0.002071 \\
11 & 6 & 0 & 2.547625 & 1.125255 & 0.000815 \\
12 & 6 & 0 & 1.133737 & 1.054229 & 0.000660 \\
13 & 6 & 0 & 0.482529 & -0.224589 & -0.000351 \\
14 & 6 & 0 & 0.368794 & 2.242793 & 0.001002 \\
15 & 1 & 0 & -0.729188 & 2.168466 & 0.000839 \\
16 & 6 & 0 & 0.987659 & 3.476093 & 0.001266 \\
17 & 6 & 0 & 2.391605 & 3.553491 & 0.001320 \\
18 & 6 & 0 & 3.155079 & 2.402492 & 0.001253 \\
19 & 1 & 0 & 0.710606 & -2.357579 & -0.003394 \\
20 & 1 & 0 & 5.332065 & -3.462699 & -0.003027 \\
21 & 1 & 0 & 2.834276 & -3.503898 & -0.006443 \\
& & & $S 45$ & &
\end{tabular}




$\begin{array}{rrrrrr}22 & 1 & 0 & 5.285306 & 0.843669 & 0.003318 \\ 23 & 1 & 0 & 6.536844 & -1.282946 & 0.002518 \\ 24 & 1 & 0 & 0.392106 & 4.400599 & 0.001415 \\ 25 & 1 & 0 & 2.878529 & 4.539978 & 0.001485 \\ 26 & 1 & 0 & 4.251486 & 2.489244 & 0.001227\end{array}$

4,4,5,5-tetramethyl-2-(2-phenanthrenyl)-1,3,2-dioxaborolane

(phenanthrene-2-Bpin) ( $C_{1}$ symmetry)

$\mathrm{E}(\mathrm{B} 3 \mathrm{LYP} / 6-31 \mathrm{G}(\mathrm{d}))=-950.23175853$ hartree

\begin{tabular}{|c|c|c|c|c|c|}
\hline \multirow{2}{*}{$\begin{array}{l}\text { Center } \\
\text { Number }\end{array}$} & \multirow{2}{*}{$\begin{array}{l}\text { Atomic } \\
\text { Number }\end{array}$} & \multirow{2}{*}{$\begin{array}{l}\text { Atomic } \\
\text { Type }\end{array}$} & \multicolumn{3}{|c|}{ Coordinates (Angstroms) } \\
\hline & & & $X$ & $\mathrm{Y}$ & $\mathrm{Z}$ \\
\hline 1 & 5 & 0 & -2.116782 & -1.168987 & -0.004921 \\
\hline 2 & 8 & 0 & -2.829925 & -0.630959 & 1.227152 \\
\hline 3 & 8 & 0 & -2.828377 & -0.623049 & -1.234771 \\
\hline 4 & 6 & 0 & -0.637545 & -0.740676 & -0.002127 \\
\hline 5 & 6 & 0 & 0.364106 & -1.690869 & -0.011030 \\
\hline 6 & 6 & 0 & 1.727838 & -1.315343 & -0.008621 \\
\hline 7 & 6 & 0 & 2.078822 & 0.067701 & 0.003254 \\
\hline 8 & 6 & 0 & 1.028959 & 1.016133 & 0.012121 \\
\hline 9 & 6 & 0 & -0.297680 & 0.625175 & 0.009528 \\
\hline 10 & 6 & 0 & 2.761112 & -2.311561 & -0.018048 \\
\hline 11 & 6 & 0 & 3.487881 & 0.441631 & 0.005702 \\
\hline 12 & 6 & 0 & 4.478475 & -0.585382 & -0.004032 \\
\hline 13 & 6 & 0 & 4.075161 & -1.962840 & -0.015903 \\
\hline 14 & 6 & 0 & 5.848964 & -0.235307 & -0.001826 \\
\hline 15 & 1 & 0 & 6.588780 & -1.032518 & -0.009382 \\
\hline 16 & 6 & 0 & 6.247640 & 1.086498 & 0.009648 \\
\hline 17 & 6 & 0 & 5.275221 & 2.104099 & 0.019350 \\
\hline 18 & 6 & 0 & 3.929298 & 1.785818 & 0.017396 \\
\hline 19 & 1 & 0 & 2.466734 & -3.358346 & -0.027021 \\
\hline 20 & 1 & 0 & 0.116950 & -2.750001 & -0.020112 \\
\hline 21 & 1 & 0 & 1.258138 & 2.076360 & 0.021135 \\
\hline 22 & 1 & 0 & -1.081015 & 1.378337 & 0.016519 \\
\hline 23 & 1 & 0 & 4.849861 & -2.725915 & -0.023170 \\
\hline 24 & 1 & 0 & 7.303817 & 1.341565 & 0.011221 \\
\hline 25 & 1 & 0 & 5.582019 & 3.146551 & 0.028457 \\
\hline 26 & 1 & 0 & 3.204542 & 2.592884 & 0.025114 \\
\hline 27 & 6 & 0 & -4.041282 & 0.162249 & 0.758646 \\
\hline 28 & 6 & 0 & -4.000948 & 0.224133 & -0.761538 \\
\hline 29 & 6 & 0 & -5.321009 & -0.298640 & -1.357981 \\
\hline 30 & 1 & 0 & -6.132922 & 0.306555 & -1.012385 \\
\hline 31 & 1 & 0 & -5.272255 & -0.253974 & -2.425936 \\
\hline 32 & 1 & 0 & -5.475036 & -1.311727 & -1.050035 \\
\hline 33 & 6 & 0 & -3.835255 & 1.680161 & -1.234961 \\
\hline 34 & 1 & 0 & -3.339198 & 1.692713 & -2.182943 \\
\hline 35 & 1 & 0 & -4.798590 & 2.135977 & -1.330442 \\
\hline 36 & 1 & 0 & -3.252853 & 2.223448 & -0.520435 \\
\hline 37 & 6 & 0 & -5.340511 & -0.515524 & 1.232173 \\
\hline 38 & 1 & 0 & -5.332670 & -0.595507 & 2.299151 \\
\hline 39 & 1 & 0 & -6.181165 & 0.070657 & 0.924614 \\
\hline 40 & 1 & 0 & -5.410408 & -1.492643 & 0.801763 \\
\hline 41 & 6 & 0 & -4.025638 & 1.580287 & 1.359082 \\
\hline 42 & 1 & 0 & -4.891917 & 2.115999 & 1.031251 \\
\hline 43 & 1 & 0 & -4.029377 & 1.515439 & 2.427109 \\
\hline 44 & 1 & 0 & -3.144751 & 2.094683 & 1.036073 \\
\hline
\end{tabular}


4,4,5,5-tetramethyl-2-(3-phenanthrenyl)-1,3,2-dioxaborolane

(phenanthrene-3-Bpin) ( $C_{1}$ symmetry)

$\mathrm{E}(\mathrm{B} 3 \mathrm{LYP} / 6-31 \mathrm{G}(\mathrm{d}))=-950.23184035$ hartree

\begin{tabular}{|c|c|c|c|c|c|}
\hline \multirow{2}{*}{$\begin{array}{l}\text { Center } \\
\text { Number }\end{array}$} & \multirow{2}{*}{$\begin{array}{l}\text { Atomic } \\
\text { Number }\end{array}$} & \multirow{2}{*}{$\begin{array}{l}\text { Atomic } \\
\text { Type }\end{array}$} & \multicolumn{3}{|c|}{ Coordinates (Angstroms) } \\
\hline & & & $\mathrm{X}$ & $\mathrm{Y}$ & $\mathrm{Z}$ \\
\hline 1 & 5 & 0 & 1.699474 & -0.924984 & 0.006182 \\
\hline 2 & 8 & 0 & 2.547742 & -0.634375 & 1.236196 \\
\hline 3 & 8 & 0 & 2.549766 & -0.650118 & -1.225689 \\
\hline 4 & 6 & 0 & 0.563008 & 1.360227 & -0.012676 \\
\hline 5 & 6 & 0 & -0.573271 & 2.144495 & -0.019120 \\
\hline 6 & 6 & 0 & -1.862724 & 1.563052 & -0.014157 \\
\hline 7 & 6 & 0 & -1.996088 & 0.142415 & -0.002238 \\
\hline 8 & 6 & 0 & -0.812445 & -0.632676 & 0.004073 \\
\hline 9 & 6 & 0 & 0.437977 & -0.041709 & -0.000991 \\
\hline 10 & 6 & 0 & -3.037351 & 2.387917 & -0.021039 \\
\hline 11 & 6 & 0 & -3.330572 & -0.444446 & 0.002854 \\
\hline 12 & 6 & 0 & -4.467782 & 0.417430 & -0.004405 \\
\hline 13 & 6 & 0 & -4.281852 & 1.840623 & -0.016427 \\
\hline 14 & 6 & 0 & -5.767838 & -0.139910 & 0.000380 \\
\hline 15 & 1 & 0 & -6.621812 & 0.533608 & -0.005314 \\
\hline 16 & 6 & 0 & -5.957772 & -1.507401 & 0.012022 \\
\hline 17 & 6 & 0 & -4.839972 & -2.362782 & 0.019291 \\
\hline 18 & 6 & 0 & -3.559281 & -1.840645 & 0.014791 \\
\hline 19 & 1 & 0 & -2.908028 & 3.467588 & -0.030102 \\
\hline 20 & 1 & 0 & 1.547549 & 1.819832 & -0.016595 \\
\hline 21 & 1 & 0 & -0.492510 & 3.229081 & -0.028191 \\
\hline 22 & 1 & 0 & -0.875278 & -1.715570 & 0.013040 \\
\hline 23 & 1 & 0 & -5.165025 & 2.475031 & -0.021778 \\
\hline 24 & 1 & 0 & -6.961942 & -1.922374 & 0.015590 \\
\hline 25 & 1 & 0 & -4.982236 & -3.440088 & 0.028515 \\
\hline 26 & 1 & 0 & -2.718666 & -2.526224 & 0.020673 \\
\hline 27 & 6 & 0 & 3.951846 & -0.289377 & -0.756543 \\
\hline 28 & 6 & 0 & 3.931748 & -0.212991 & 0.763383 \\
\hline 29 & 6 & 0 & 4.244800 & 1.219961 & 1.232690 \\
\hline 30 & 1 & 0 & 3.719637 & 1.419773 & 2.143283 \\
\hline 31 & 1 & 0 & 5.297182 & 1.318959 & 1.398799 \\
\hline 32 & 1 & 0 & 3.935092 & 1.916771 & 0.482067 \\
\hline 33 & 6 & 0 & 5.011336 & -1.132360 & 1.364092 \\
\hline 34 & 1 & 0 & 5.973747 & -0.836015 & 1.002369 \\
\hline 35 & 1 & 0 & 4.992087 & -1.055204 & 2.431133 \\
\hline 36 & 1 & 0 & 4.818275 & -2.144643 & 1.076151 \\
\hline 37 & 6 & 0 & 4.962990 & -1.352072 & -1.225446 \\
\hline 38 & 1 & 0 & 4.926838 & -1.432823 & -2.291782 \\
\hline 39 & 1 & 0 & 5.948300 & -1.065005 & -0.922697 \\
\hline 40 & 1 & 0 & 4.716379 & -2.296753 & -0.787655 \\
\hline 41 & 6 & 0 & 4.395983 & 1.055757 & -1.360660 \\
\hline 42 & 1 & 0 & 5.383188 & 1.290565 & -1.021261 \\
\hline 43 & 1 & 0 & 4.393179 & 0.985507 & -2.428347 \\
\hline 44 & 1 & 0 & 3.720169 & 1.825805 & -1.052113 \\
\hline
\end{tabular}

$9 \mathrm{H}$-fluorene (1g) ( $C_{2 \mathrm{v}}$ symmetry)

$\mathrm{E}(\mathrm{B} 3 \mathrm{LYP} / 6-31 \mathrm{G}(\mathrm{d}))=-501.42318748$ hartree 


\begin{tabular}{|c|c|c|c|c|c|}
\hline \multirow{2}{*}{$\begin{array}{l}\text { Center } \\
\text { Number }\end{array}$} & \multirow{2}{*}{$\begin{array}{l}\text { Atomic } \\
\text { Number }\end{array}$} & \multirow{2}{*}{$\begin{array}{l}\text { Atomic } \\
\text { Type }\end{array}$} & \multicolumn{3}{|c|}{ Coordinates (Angstroms) } \\
\hline & & & $\mathrm{X}$ & $\mathrm{Y}$ & Z \\
\hline 1 & 6 & 0 & 0.000000 & 3.016572 & -1.211825 \\
\hline 2 & 6 & 0 & 0.000000 & 3.461862 & 0.114691 \\
\hline 3 & 6 & 0 & 0.000000 & 2.545252 & 1.172284 \\
\hline 4 & 6 & 0 & 0.000000 & 1.184674 & 0.886686 \\
\hline 5 & 6 & 0 & 0.000000 & 0.734801 & -0.450353 \\
\hline 6 & 6 & 0 & 0.000000 & 1.651189 & -1.504434 \\
\hline 7 & 1 & 0 & 0.000000 & 3.740628 & -2.022382 \\
\hline 8 & 1 & 0 & 0.000000 & 4.527990 & 0.325383 \\
\hline 9 & 1 & 0 & 0.000000 & 1.311931 & -2.537298 \\
\hline 10 & 6 & 0 & 0.000000 & -1.184674 & 0.886686 \\
\hline 11 & 6 & 0 & 0.000000 & -2.545252 & 1.172284 \\
\hline 12 & 6 & 0 & 0.000000 & -3.461862 & 0.114691 \\
\hline 13 & 6 & 0 & 0.000000 & -3.016572 & -1.211825 \\
\hline 14 & 6 & 0 & 0.000000 & -1.651189 & -1.504434 \\
\hline 15 & 6 & 0 & 0.000000 & -0.734801 & -0.450353 \\
\hline 16 & 1 & 0 & 0.000000 & -2.897559 & 2.201345 \\
\hline 17 & 1 & 0 & 0.000000 & -4.527990 & 0.325383 \\
\hline 18 & 1 & 0 & 0.000000 & -3.740628 & -2.022382 \\
\hline 19 & 1 & 0 & 0.000000 & -1.311931 & -2.537298 \\
\hline 20 & 1 & 0 & 0.000000 & 2.897559 & 2.201345 \\
\hline 21 & 6 & 0 & 0.000000 & 0.000000 & 1.832732 \\
\hline 22 & 1 & 0 & 0.878901 & 0.000000 & 2.492467 \\
\hline 23 & 1 & 0 & -0.878901 & 0.000000 & 2.492467 \\
\hline
\end{tabular}

diiodo-(9H-fluoren-1-yl)borane (fluorene-1-BI 2$)\left(C_{1}\right.$ symmetry)

$\mathrm{E}(\mathrm{B} 3 \mathrm{LYP} / 631 \mathrm{LAN})=-548.50006823$ hartree

\begin{tabular}{rrrrrr} 
Center & Atomic & Atomic & \multicolumn{2}{c}{ Coordinates (Angstroms) } \\
Number & Number & Type & X & Y & Z \\
\hline 1 & 5 & 0 & 1.320738 & -0.000031 & 0.254666 \\
2 & 53 & 0 & 2.266363 & -1.913777 & -0.317584 \\
3 & 53 & 0 & 2.266105 & 1.914055 & -0.316872 \\
4 & 6 & 0 & -1.259951 & -0.000799 & 3.239326 \\
5 & 6 & 0 & -0.067049 & -0.000532 & 2.507948 \\
6 & 6 & 0 & -0.091087 & -0.000285 & 1.108627 \\
7 & 6 & 0 & -1.317717 & -0.000221 & 0.454319 \\
8 & 6 & 0 & -2.520887 & -0.000402 & 1.190824 \\
9 & 6 & 0 & -2.494764 & -0.000708 & 2.587310 \\
10 & 1 & 0 & -1.225002 & -0.001021 & 4.325621 \\
11 & 1 & 0 & 0.885712 & -0.000518 & 3.030697 \\
12 & 1 & 0 & -3.417874 & -0.000969 & 3.161568 \\
13 & 6 & 0 & -3.134569 & 0.000033 & -1.066495 \\
14 & 6 & 0 & -3.994549 & 0.000454 & -2.158786 \\
15 & 6 & 0 & -5.376267 & 0.000573 & -1.936159 \\
16 & 6 & 0 & -5.886274 & 0.000298 & -0.633168 \\
17 & 6 & 0 & -5.027083 & -0.000165 & 0.467614 \\
18 & 6 & 0 & -3.647820 & -0.000245 & 0.247535 \\
19 & 1 & 0 & -3.604206 & 0.000560 & -3.174031 \\
20 & 1 & 0 & -6.058529 & 0.000969 & -2.782058 \\
21 & 1 & 0 & -6.961758 & 0.000464 & -0.476360 \\
22 & 1 & 0 & -5.429924 & -0.000523 & 1.477380 \\
23 & 6 & 0 & -1.618896 & -0.000126 & -1.031517 \\
24 & 1 & 0 & -1.195448 & 0.879043 & -1.537280 \\
& & & $S 48$ & & \\
& & 0 & & &
\end{tabular}


diiodo-(9H-fluoren-2-yl)borane (fluorene-2-BI $)$ ( $C_{1}$ symmetry)

$\mathrm{E}(\mathrm{B} 3 \mathrm{LYP} / 631 \mathrm{LAN})=-548.50781878$ hartree

\begin{tabular}{|c|c|c|c|c|c|}
\hline \multirow{2}{*}{$\begin{array}{l}\text { Center } \\
\text { Number }\end{array}$} & \multirow{2}{*}{$\begin{array}{l}\text { Atomic } \\
\text { Number }\end{array}$} & \multirow{2}{*}{$\begin{array}{l}\text { Atomic } \\
\text { Type }\end{array}$} & \multicolumn{3}{|c|}{ Coordinates (Angstroms) } \\
\hline & & & $\mathrm{X}$ & $\mathrm{Y}$ & $\mathrm{Z}$ \\
\hline 1 & 5 & 0 & 1.675226 & -0.000143 & 0.070362 \\
\hline 2 & 53 & 0 & 2.774588 & -1.913564 & -0.049327 \\
\hline 3 & 53 & 0 & 2.773308 & 1.914266 & -0.045197 \\
\hline 4 & 6 & 0 & -0.526138 & -0.004899 & 1.527984 \\
\hline 5 & 6 & 0 & 0.034601 & -0.000882 & 0.245999 \\
\hline 6 & 6 & 0 & -0.784992 & 0.002511 & -0.888431 \\
\hline 7 & 6 & 0 & -2.165481 & 0.001923 & -0.724150 \\
\hline 8 & 6 & 0 & -2.731715 & -0.002036 & 0.567911 \\
\hline 9 & 6 & 0 & -1.912032 & -0.005479 & 1.698823 \\
\hline 10 & 1 & 0 & 0.123478 & -0.007535 & 2.399334 \\
\hline 11 & 1 & 0 & -2.341212 & -0.008660 & 2.697672 \\
\hline 12 & 6 & 0 & -4.525568 & 0.002470 & -0.933478 \\
\hline 13 & 6 & 0 & -5.855569 & 0.003877 & -1.338151 \\
\hline 14 & 6 & 0 & -6.862045 & 0.001076 & -0.365677 \\
\hline 15 & 6 & 0 & -6.535712 & -0.003058 & 0.994978 \\
\hline 16 & 6 & 0 & -5.201504 & -0.004529 & 1.407069 \\
\hline 17 & 6 & 0 & -4.195586 & -0.001699 & 0.438089 \\
\hline 18 & 1 & 0 & -6.115610 & 0.006978 & -2.394304 \\
\hline 19 & 1 & 0 & -7.905383 & 0.002207 & -0.669766 \\
\hline 20 & 1 & 0 & -7.328538 & -0.005128 & 1.738400 \\
\hline 21 & 1 & 0 & -4.954850 & -0.007891 & 2.465870 \\
\hline 22 & 1 & 0 & -0.343121 & 0.005586 & -1.882327 \\
\hline 23 & 6 & 0 & -3.261928 & 0.004878 & -1.771152 \\
\hline 24 & 1 & 0 & -3.203694 & 0.885936 & -2.425667 \\
\hline 25 & 1 & 0 & -3.203681 & -0.872899 & -2.430134 \\
\hline
\end{tabular}

diiodo-(9H-fluoren-3-yl)borane (fluorene-3-BI $)_{2}\left(C_{1}\right.$ symmetry)

$\mathrm{E}(\mathrm{B} 3 \mathrm{LYP} / 631 \mathrm{LAN})=-548.50627930$ hartree

\begin{tabular}{rrrrrr}
$\begin{array}{c}\text { Center } \\
\text { Number }\end{array}$ & $\begin{array}{c}\text { Atomic } \\
\text { Number }\end{array}$ & $\begin{array}{c}\text { Atomic } \\
\text { Type }\end{array}$ & \multicolumn{3}{c}{ Cordinates (Angstroms) } \\
-1 & 5 & 0 & 1.565118 & -0.000341 & Z \\
2 & 53 & 0 & 2.606327 & 1.915344 & -0.230289 \\
3 & 53 & 0 & 2.609378 & -1.912474 & -0.240177 \\
4 & 6 & 0 & 0.008093 & -0.002993 & 0.676500 \\
5 & 6 & 0 & -0.243158 & -0.009281 & 2.053003 \\
6 & 6 & 0 & -1.556736 & -0.011578 & 2.535897 \\
7 & 6 & 0 & -2.609845 & -0.007610 & 1.628327 \\
8 & 6 & 0 & -2.357520 & -0.001360 & 0.240396 \\
9 & 6 & 0 & -1.045834 & 0.000984 & -0.239529 \\
10 & 1 & 0 & 0.588372 & -0.012403 & 2.752695 \\
11 & 1 & 0 & -0.843674 & 0.005917 & -1.307710 \\
12 & 6 & 0 & -4.684129 & -0.002742 & 0.483271 \\
13 & 6 & 0 & -6.013265 & -0.001310 & 0.075765 \\
14 & 6 & 0 & -6.304619 & 0.004586 & -1.293097 \\
15 & 6 & 0 & -5.273722 & 0.008955 & -2.239209 \\
16 & 6 & 0 & -3.936953 & 0.007582 & -1.835505 \\
17 & 6 & 0 & -3.644110 & 0.001662 & -0.469852 \\
& & & & &
\end{tabular}




$\begin{array}{rrrrrr}18 & 1 & 0 & -6.819044 & -0.004577 & 0.806381 \\ 19 & 1 & 0 & -7.339812 & 0.005692 & -1.623853 \\ 20 & 1 & 0 & -5.515868 & 0.013445 & -3.298737 \\ 21 & 1 & 0 & -3.140796 & 0.011144 & -2.575796 \\ 22 & 1 & 0 & -1.745604 & -0.016430 & 3.607062 \\ 23 & 6 & 0 & -4.104179 & -0.008751 & 1.884025 \\ 24 & 1 & 0 & -4.422946 & -0.890428 & 2.457679 \\ 25 & 1 & 0 & -4.422714 & 0.868399 & 2.464789\end{array}$

diiodo-(9H-fluoren-4-yl)borane (fluorene-4-BI 2$)\left(C_{1}\right.$ symmetry)

$\mathrm{E}(\mathrm{B} 3 \mathrm{LYP} / 631 \mathrm{LAN})=-548.49523373$ hartree

\begin{tabular}{|c|c|c|c|c|c|}
\hline \multirow{2}{*}{$\begin{array}{l}\text { Center } \\
\text { Number }\end{array}$} & \multirow{2}{*}{$\begin{array}{l}\text { Atomic } \\
\text { Number }\end{array}$} & \multirow{2}{*}{$\begin{array}{l}\text { Atomic } \\
\text { Type }\end{array}$} & \multicolumn{3}{|c|}{ Coordinates (Angstroms) } \\
\hline & & & $X$ & $\mathrm{Y}$ & $\mathrm{Z}$ \\
\hline 1 & 5 & 0 & 0.972566 & 0.414595 & -0.004821 \\
\hline 2 & 53 & 0 & 1.863931 & -0.254842 & -1.913072 \\
\hline 3 & 53 & 0 & 1.872055 & -0.210889 & 1.914499 \\
\hline 4 & 6 & 0 & -0.228441 & 2.771065 & -0.026734 \\
\hline 5 & 6 & 0 & -1.356963 & 3.598299 & -0.033886 \\
\hline 6 & 6 & 0 & -2.643048 & 3.046372 & -0.027590 \\
\hline 7 & 6 & 0 & -2.784322 & 1.663405 & -0.014031 \\
\hline 8 & 6 & 0 & -1.647167 & 0.828609 & -0.006724 \\
\hline 9 & 6 & 0 & -0.364496 & 1.381392 & -0.013086 \\
\hline 10 & 1 & 0 & 0.763783 & 3.214602 & -0.031795 \\
\hline 11 & 1 & 0 & -1.233784 & 4.677992 & -0.044415 \\
\hline 12 & 6 & 0 & -3.504189 & -0.593830 & 0.008514 \\
\hline 13 & 6 & 0 & -4.189647 & -1.803233 & 0.021040 \\
\hline 14 & 6 & 0 & -3.460539 & -2.997796 & 0.032357 \\
\hline 15 & 6 & 0 & -2.061452 & -2.976613 & 0.031123 \\
\hline 16 & 6 & 0 & -1.367837 & -1.764726 & 0.018526 \\
\hline 17 & 6 & 0 & -2.093657 & -0.571471 & 0.007259 \\
\hline 18 & 1 & 0 & -5.277103 & -1.826252 & 0.021955 \\
\hline 19 & 1 & 0 & -3.985217 & -3.949448 & 0.042270 \\
\hline 20 & 1 & 0 & -1.509190 & -3.912657 & 0.040074 \\
\hline 21 & 1 & 0 & -0.280721 & -1.755351 & 0.017491 \\
\hline 22 & 1 & 0 & -3.516408 & 3.694677 & -0.033243 \\
\hline 23 & 6 & 0 & -4.045572 & 0.822224 & -0.005093 \\
\hline 24 & 1 & 0 & -4.673519 & 1.031227 & 0.872428 \\
\hline 25 & 1 & 0 & -4.673951 & 1.013824 & -0.886327 \\
\hline
\end{tabular}

2-(9H-fluoren-2-yl)-4,4,5,5-tetramethyl-1,3,2-dioxaborolane (fluorene-2-Bpin) $\left(C_{1}\right.$ symmetry) $\mathrm{E}(\mathrm{B} 3 \mathrm{LYP} / 6-31 \mathrm{G}(\mathrm{d}))=-912.11656938$ hartree

\begin{tabular}{cccccc}
$\begin{array}{c}\text { Center } \\
\text { Number }\end{array}$ & $\begin{array}{c}\text { Atomic } \\
\text { Number }\end{array}$ & $\begin{array}{c}\text { Atomic } \\
\text { Type }\end{array}$ & \multicolumn{2}{c}{ Coordinates (Angstroms) } \\
-1 & 5 & 0 & -1.906560 & -1.148712 & -0.012541 \\
2 & 8 & 0 & -2.645871 & -0.655045 & 1.222677 \\
3 & 8 & 0 & -2.643844 & -0.630224 & -1.239133 \\
4 & 6 & 0 & 5.788069 & 1.545697 & 0.022694 \\
5 & 6 & 0 & 6.409312 & 0.292040 & 0.004569 \\
6 & 6 & 0 & 5.644757 & -0.880082 & -0.011618 \\
7 & 6 & 0 & 4.258004 & -0.781847 & -0.009530 \\
8 & 6 & 0 & 3.630791 & 0.481613 & 0.008649 \\
9 & 6 & 0 & 4.395604 & 1.650225 & 0.024847 \\
& & 0 & S50 & &
\end{tabular}




\begin{tabular}{|c|c|c|c|c|c|}
\hline 10 & 1 & 0 & 6.395388 & 2.446956 & 0.035162 \\
\hline 11 & 1 & 0 & 7.494173 & 0.228041 & 0.003051 \\
\hline 12 & 1 & 0 & 3.919274 & 2.627370 & 0.039023 \\
\hline 13 & 6 & 0 & 1.910586 & -1.103482 & -0.012956 \\
\hline 14 & 6 & 0 & 0.601399 & -1.571088 & -0.019171 \\
\hline 15 & 6 & 0 & -0.450312 & -0.647816 & -0.005804 \\
\hline 16 & 6 & 0 & -0.189245 & 0.726725 & 0.013521 \\
\hline 17 & 6 & 0 & 1.123790 & 1.201940 & 0.019883 \\
\hline 18 & 6 & 0 & 2.174777 & 0.282132 & 0.006526 \\
\hline 19 & 1 & 0 & 0.392026 & -2.638342 & -0.034064 \\
\hline 20 & 1 & 0 & -1.016626 & 1.431419 & 0.023663 \\
\hline 21 & 1 & 0 & 1.319665 & 2.271198 & 0.035064 \\
\hline 22 & 1 & 0 & 6.133527 & -1.851677 & -0.025630 \\
\hline 23 & 6 & 0 & 3.212740 & -1.879854 & -0.024372 \\
\hline 24 & 1 & 0 & 3.301864 & -2.521022 & -0.912632 \\
\hline 25 & 1 & 0 & 3.302610 & -2.545017 & 0.846045 \\
\hline 26 & 6 & 0 & -3.894802 & 0.080487 & 0.758751 \\
\hline 27 & 6 & 0 & -3.857039 & 0.154689 & -0.760947 \\
\hline 28 & 6 & 0 & -5.149425 & -0.428619 & -1.361854 \\
\hline 29 & 1 & 0 & -5.990423 & 0.133091 & -1.012420 \\
\hline 30 & 1 & 0 & -5.102713 & -0.373965 & -2.429435 \\
\hline 31 & 1 & 0 & -5.253096 & -1.450270 & -1.061218 \\
\hline 32 & 6 & 0 & -3.763377 & 1.620334 & -1.224321 \\
\hline 33 & 1 & 0 & -3.271979 & 1.663499 & -2.173828 \\
\hline 34 & 1 & 0 & -4.747884 & 2.029882 & -1.313286 \\
\hline 35 & 1 & 0 & -3.205191 & 2.185956 & -0.507802 \\
\hline 36 & 6 & 0 & -5.159095 & -0.663919 & 1.226748 \\
\hline 37 & 1 & 0 & -5.147717 & -0.750660 & 2.293166 \\
\hline 38 & 1 & 0 & -6.027598 & -0.117961 & 0.922557 \\
\hline 39 & 1 & 0 & -5.180408 & -1.640354 & 0.789689 \\
\hline 40 & 6 & 0 & -3.949507 & 1.493423 & 1.368849 \\
\hline 41 & 1 & 0 & -4.839783 & 1.988847 & 1.041951 \\
\hline 42 & 1 & 0 & -3.953093 & 1.421064 & 2.436394 \\
\hline 43 & 1 & 0 & -3.093654 & 2.052071 & 1.052103 \\
\hline
\end{tabular}

3-(9H-fluoren-2-yl)-4,4,5,5-tetramethyl-1,3,2-dioxaborolane (fluorene-3-Bpin) $\left(C_{1}\right.$ symmetry) $\mathrm{E}(\mathrm{B} 3 \mathrm{LYP} / 6-31 \mathrm{G}(\mathrm{d}))=-912.11610620$ hartree

\begin{tabular}{|c|c|c|c|c|c|}
\hline \multirow{2}{*}{$\begin{array}{l}\text { Center } \\
\text { Number }\end{array}$} & \multirow{2}{*}{$\begin{array}{l}\text { Atomic } \\
\text { Number }\end{array}$} & \multirow{2}{*}{$\begin{array}{l}\text { Atomic } \\
\text { Type }\end{array}$} & \multicolumn{3}{|c|}{ Coordinates (Angstroms) } \\
\hline & & & $X$ & $\mathrm{Y}$ & Z \\
\hline 1 & 5 & 0 & 1.620790 & -0.975901 & 0.010360 \\
\hline 2 & 8 & 0 & 2.453176 & -0.636956 & 1.238853 \\
\hline 3 & 8 & 0 & 2.455434 & -0.662271 & -1.222951 \\
\hline 4 & 6 & 0 & -5.478550 & -1.841376 & 0.022716 \\
\hline 5 & 6 & 0 & -6.276089 & -0.691750 & 0.007913 \\
\hline 6 & 6 & 0 & -5.690760 & 0.579391 & -0.008421 \\
\hline 7 & 6 & 0 & -4.304512 & 0.684524 & -0.009829 \\
\hline 8 & 6 & 0 & -3.499638 & -0.473930 & 0.004998 \\
\hline 9 & 6 & 0 & -4.085734 & -1.741636 & 0.021349 \\
\hline 10 & 1 & 0 & -5.947852 & -2.821609 & 0.035344 \\
\hline 11 & 1 & 0 & -7.358680 & -0.786708 & 0.009113 \\
\hline 12 & 1 & 0 & -3.471908 & -2.638847 & 0.032954 \\
\hline 13 & 6 & 0 & -2.029151 & 1.345195 & -0.018465 \\
\hline 14 & 6 & 0 & -0.802211 & 1.998806 & -0.027168 \\
\hline 15 & 6 & 0 & 0.372976 & 1.238837 & -0.017685 \\
\hline 16 & 6 & 0 & 0.315286 & -0.159105 & 0.000276 \\
\hline 17 & 6 & 0 & -0.914350 & -0.820805 & 0.009123 \\
\hline & & & S51 & & \\
\hline
\end{tabular}




$\begin{array}{rrrrrr}18 & 6 & 0 & -2.088316 & -0.064158 & -0.000357 \\ 19 & 1 & 0 & -0.750820 & 3.085203 & -0.041018 \\ 20 & 1 & 0 & 1.338044 & 1.738461 & -0.024411 \\ 21 & 1 & 0 & -0.952095 & -1.907215 & 0.023226 \\ 22 & 1 & 0 & -6.316083 & 1.469296 & -0.019840 \\ 23 & 6 & 0 & -3.430663 & 1.923296 & -0.025605 \\ 24 & 1 & 0 & -3.614687 & 2.545562 & -0.912716 \\ 25 & 1 & 0 & -3.614345 & 2.567287 & 0.845991 \\ 26 & 6 & 0 & 3.837085 & -0.227635 & -0.755838 \\ 27 & 6 & 0 & 3.813418 & -0.146404 & 0.763785 \\ 28 & 6 & 0 & 4.052030 & 1.302661 & 1.227332 \\ 29 & 1 & 0 & 3.516085 & 1.479136 & 2.136463 \\ 30 & 1 & 0 & 5.097733 & 1.456132 & 1.394218 \\ 31 & 1 & 0 & 3.708059 & 1.979532 & 0.473389 \\ 32 & 6 & 0 & 4.210896 & 1.136284 & -1.365420 \\ 33 & 1 & 0 & 5.178732 & 1.429854 & -1.016119 \\ 34 & 1 & 0 & 4.225392 & 1.058125 & -2.432463 \\ 35 & 1 & 0 & 3.488290 & 1.868531 & -1.071217 \\ 36 & 6 & 0 & 4.939263 & -1.006319 & 1.367623 \\ 37 & 1 & 0 & 5.886766 & -0.642255 & 1.029103 \\ 38 & 1 & 0 & 4.896708 & -0.951019 & 2.435345 \\ 39 & 1 & 0 & 4.816559 & -2.023156 & 1.057969 \\ 40 & 6 & 0 & 4.901741 & -1.238437 & -1.221013 \\ 41 & 1 & 0 & 4.876554 & -1.317426 & -2.287796 \\ 42 & 1 & 0 & 5.869926 & -0.904810 & -0.910832 \\ 43 & 1 & 0 & 4.698472 & -2.195384 & -0.787615 \\ -------------------------------------------------------------------------\end{array}$

triphenylene (1h) ( $D_{3 \mathrm{~h}}$ symmetry)

$\mathrm{E}(\mathrm{B} 3 \mathrm{LYP} / 6-31 \mathrm{G}(\mathrm{d}))=-693.18106547$ hartree

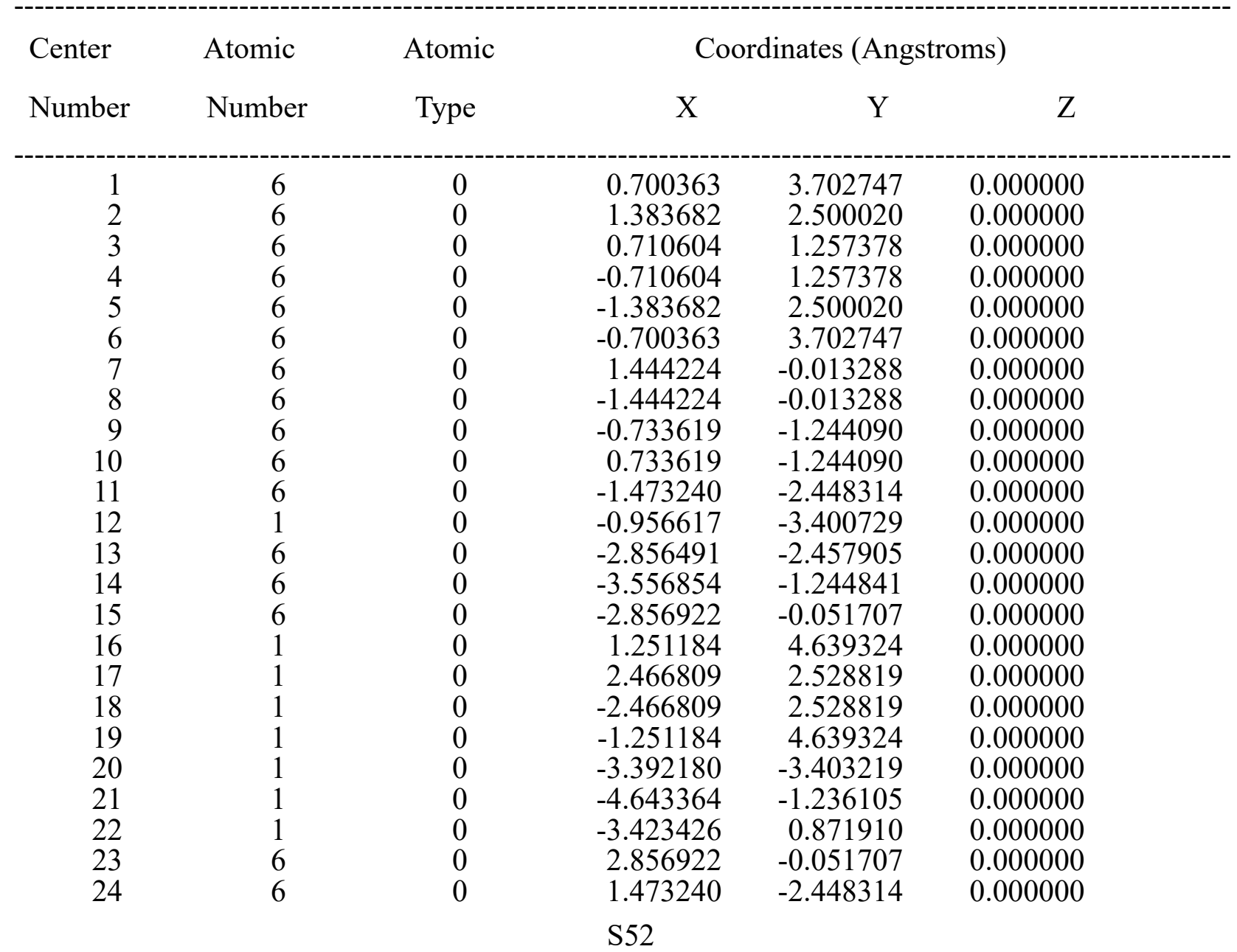




$\begin{array}{rrrrrr}25 & 6 & 0 & 2.856491 & -2.457905 & 0.000000 \\ 26 & 1 & 0 & 3.392180 & -3.403219 & 0.000000 \\ 27 & 6 & 0 & 3.556854 & -1.244841 & 0.000000 \\ 28 & 1 & 0 & 4.643364 & -1.236105 & 0.000000 \\ 29 & 1 & 0 & 3.423426 & 0.871910 & 0.000000 \\ 30 & 1 & 0 & 0.956617 & -3.400729 & 0.000000\end{array}$

diiodo-(triphenylen-1-yl)borane (triphenylene-1-BI $\left.\mathbf{I}_{2}\right)\left(C_{1}\right.$ symmetry)

$\mathrm{E}(\mathrm{B} 3 \mathrm{LYP} / 631 \mathrm{LAN})=-740.24633656$ hartree

\begin{tabular}{|c|c|c|c|c|c|}
\hline \multirow{2}{*}{$\begin{array}{l}\text { Center } \\
\text { Number }\end{array}$} & \multirow{2}{*}{$\begin{array}{l}\text { Atomic } \\
\text { Number }\end{array}$} & \multirow{2}{*}{$\begin{array}{c}\text { Atomic } \\
\text { Type }\end{array}$} & \multicolumn{3}{|c|}{ Coordinates (Angstroms) } \\
\hline & & & $\mathrm{X}$ & Y & $\mathrm{Z}$ \\
\hline 1 & 5 & 0 & -1.604394 & -0.545924 & -0.000134 \\
\hline 2 & 53 & 0 & -2.555851 & 0.014507 & 1.914218 \\
\hline 3 & 53 & 0 & -2.556779 & 0.015911 & -1.913613 \\
\hline 4 & 6 & 0 & -0.040656 & 2.943246 & 0.000421 \\
\hline 5 & 6 & 0 & -0.079550 & 1.560508 & 0.000203 \\
\hline 6 & 6 & 0 & 1.098011 & 0.779136 & -0.000094 \\
\hline 7 & 6 & 0 & 2.353265 & 1.446157 & -0.000047 \\
\hline 8 & 6 & 0 & 2.364224 & 2.859238 & 0.000082 \\
\hline 9 & 6 & 0 & 1.196285 & 3.600519 & 0.000309 \\
\hline 10 & 6 & 0 & 1.046405 & -0.687076 & -0.000548 \\
\hline 11 & 6 & 0 & 3.597400 & 0.668458 & -0.000050 \\
\hline 12 & 6 & 0 & 3.547459 & -0.752153 & -0.000349 \\
\hline 13 & 6 & 0 & 2.251755 & -1.440663 & -0.000729 \\
\hline 14 & 6 & 0 & 4.765879 & -1.468198 & -0.000197 \\
\hline 15 & 1 & 0 & 4.756435 & -2.551557 & -0.000304 \\
\hline 16 & 6 & 0 & 5.991848 & -0.827479 & 0.000170 \\
\hline 17 & 6 & 0 & 6.040976 & 0.572346 & 0.000458 \\
\hline 18 & 6 & 0 & 4.862933 & 1.297374 & 0.000376 \\
\hline 19 & 1 & 0 & -0.966615 & 3.511737 & 0.000655 \\
\hline 20 & 1 & 0 & -1.049092 & 1.077123 & 0.000284 \\
\hline 21 & 1 & 0 & 3.307170 & 3.392736 & -0.000014 \\
\hline 22 & 1 & 0 & 1.243181 & 4.686060 & 0.000409 \\
\hline 23 & 1 & 0 & 6.908518 & -1.410816 & 0.000283 \\
\hline 24 & 1 & 0 & 6.996211 & 1.090134 & 0.000799 \\
\hline 25 & 1 & 0 & 4.929407 & 2.378776 & 0.000714 \\
\hline 26 & 6 & 0 & -0.182972 & -1.383814 & -0.000786 \\
\hline 27 & 6 & 0 & 2.163254 & -2.850956 & -0.001338 \\
\hline 28 & 6 & 0 & 0.946223 & -3.508367 & -0.001764 \\
\hline 29 & 1 & 0 & 0.916928 & -4.594482 & -0.002151 \\
\hline 30 & 6 & 0 & -0.241413 & -2.765776 & -0.001046 \\
\hline 31 & 1 & 0 & -1.205058 & -3.267639 & -0.000959 \\
\hline 32 & 1 & 0 & 3.066353 & -3.449586 & -0.001539 \\
\hline
\end{tabular}

diiodo-(triphenylen-2-yl)borane (triphenylene-2- $\left.\mathbf{B I}_{2}\right)\left(C_{1}\right.$ symmetry)

$\mathrm{E}(\mathrm{B} 3 \mathrm{LYP} / 631 \mathrm{LAN})=-740.26460729$ hartree

\begin{tabular}{llcccc} 
Center & Atomic & Atomic & \multicolumn{3}{c}{ Coordinates (Angstroms) } \\
Number & Number & Type & X & Y & Z
\end{tabular}




\begin{tabular}{|c|c|c|c|c|c|}
\hline 1 & 5 & 0 & 2.287760 & -0.213764 & 0.001141 \\
\hline 2 & 53 & 0 & 3.369762 & 0.019504 & 1.913981 \\
\hline 3 & 53 & 0 & 3.371543 & -0.007181 & -1.913758 \\
\hline 4 & 6 & 0 & -5.392423 & -2.957691 & 0.014340 \\
\hline 5 & 6 & 0 & -4.036496 & -2.683944 & 0.013334 \\
\hline 6 & 6 & 0 & -3.540628 & -1.360591 & 0.006636 \\
\hline 7 & 6 & 0 & -4.473397 & -0.287983 & 0.000923 \\
\hline 8 & 6 & 0 & -5.852590 & -0.595786 & 0.002000 \\
\hline 9 & 6 & 0 & -6.311568 & -1.900728 & 0.008553 \\
\hline 10 & 6 & 0 & -2.100510 & -1.080415 & 0.005399 \\
\hline 11 & 6 & 0 & -3.996113 & 1.099404 & -0.005809 \\
\hline 12 & 6 & 0 & -2.600797 & 1.370925 & -0.006868 \\
\hline 13 & 6 & 0 & -1.637975 & 0.263750 & -0.001303 \\
\hline 14 & 6 & 0 & -2.177784 & 2.719364 & -0.013275 \\
\hline 15 & 1 & 0 & -1.120171 & 2.954330 & -0.014104 \\
\hline 16 & 6 & 0 & -3.078328 & 3.769371 & -0.018541 \\
\hline 17 & 6 & 0 & -4.453209 & 3.501742 & -0.017504 \\
\hline 18 & 6 & 0 & -4.894074 & 2.190616 & -0.011222 \\
\hline 19 & 1 & 0 & -5.737593 & -3.987940 & 0.019576 \\
\hline 20 & 1 & 0 & -3.347032 & -3.519581 & 0.017888 \\
\hline 21 & 1 & 0 & -6.584884 & 0.202647 & -0.002399 \\
\hline 22 & 1 & 0 & -7.379748 & -2.099699 & 0.009190 \\
\hline 23 & 1 & 0 & -2.716600 & 4.793917 & -0.023394 \\
\hline 24 & 1 & 0 & -5.172968 & 4.315690 & -0.021539 \\
\hline 25 & 1 & 0 & -5.962635 & 2.011655 & -0.010447 \\
\hline 26 & 6 & 0 & -1.144619 & -2.121116 & 0.010771 \\
\hline 27 & 6 & 0 & -0.244021 & 0.495377 & -0.002473 \\
\hline 28 & 6 & 0 & 0.670771 & -0.542161 & 0.002678 \\
\hline 29 & 6 & 0 & 0.214956 & -1.866586 & 0.009821 \\
\hline 30 & 1 & 0 & 0.921094 & -2.692314 & 0.014336 \\
\hline 31 & 1 & 0 & -1.470299 & -3.154469 & 0.016224 \\
\hline 32 & 1 & 0 & 0.135666 & 1.510146 & -0.007583 \\
\hline
\end{tabular}

4,4,5,5-tetramethyl-2-(2-triphenylenyl)-1,3,2-dioxaborolane

(triphenylene-2-Bpin) ( $C_{1}$ symmetry)

$\mathrm{E}(\mathrm{B} 3 \mathrm{LYP} / 6-31 \mathrm{G}(\mathrm{d}))=-1103.87437199$ hartree

\begin{tabular}{|c|c|c|c|c|c|}
\hline \multirow{2}{*}{$\begin{array}{l}\text { Center } \\
\text { Number }\end{array}$} & \multirow{2}{*}{$\begin{array}{l}\text { Atomic } \\
\text { Number }\end{array}$} & \multirow{2}{*}{$\begin{array}{l}\text { Atomic } \\
\text { Type }\end{array}$} & \multicolumn{3}{|c|}{ Coordinates (Angstroms) } \\
\hline & & & $X$ & $\mathrm{Y}$ & Z \\
\hline 1 & 5 & 0 & 2.538928 & 1.022544 & -0.002272 \\
\hline 2 & 8 & 0 & 3.328288 & 0.600187 & -1.233316 \\
\hline 3 & 8 & 0 & 3.329811 & 0.603653 & 1.228616 \\
\hline 4 & 6 & 0 & -5.304016 & -2.635282 & 0.009349 \\
\hline 5 & 6 & 0 & -5.172019 & -1.258318 & 0.004647 \\
\hline 6 & 6 & 0 & -3.907053 & -0.628199 & 0.002910 \\
\hline 7 & 6 & 0 & -2.743682 & -1.444983 & 0.006008 \\
\hline 8 & 6 & 0 & -2.907144 & -2.848612 & 0.010889 \\
\hline 9 & 6 & 0 & -4.157615 & -3.440134 & 0.012559 \\
\hline 10 & 6 & 0 & -3.777373 & 0.833171 & -0.001861 \\
\hline 11 & 6 & 0 & -1.413104 & -0.826723 & 0.004031 \\
\hline 12 & 6 & 0 & -1.287397 & 0.589188 & -0.000731 \\
\hline 13 & 6 & 0 & -2.488257 & 1.432290 & -0.003597 \\
\hline 14 & 6 & 0 & 0.010056 & 1.149439 & -0.002659 \\
\hline 15 & 1 & 0 & 0.134343 & 2.225680 & -0.006395 \\
\hline & & & S54 & & \\
\hline
\end{tabular}




\begin{tabular}{|c|c|c|c|c|c|}
\hline 16 & 6 & 0 & 1.147611 & 0.362367 & 0.000006 \\
\hline 17 & 6 & 0 & 1.023662 & -1.032818 & 0.004705 \\
\hline 18 & 6 & 0 & -0.234831 & -1.606963 & 0.006621 \\
\hline 19 & 1 & 0 & -6.293037 & -3.085190 & 0.010551 \\
\hline 20 & 1 & 0 & -6.074516 & -0.659014 & 0.002249 \\
\hline 21 & 1 & 0 & -2.037222 & -3.494364 & 0.013461 \\
\hline 22 & 1 & 0 & -4.245005 & -4.523161 & 0.016332 \\
\hline 23 & 1 & 0 & 1.907717 & -1.664498 & 0.006794 \\
\hline 24 & 1 & 0 & -0.302282 & -2.688300 & 0.010154 \\
\hline 25 & 6 & 0 & -4.911398 & 1.676255 & -0.004855 \\
\hline 26 & 6 & 0 & -2.402089 & 2.842721 & -0.008037 \\
\hline 27 & 6 & 0 & -3.528715 & 3.645255 & -0.010697 \\
\hline 28 & 1 & 0 & -3.423788 & 4.726681 & -0.014174 \\
\hline 29 & 6 & 0 & -4.798894 & 3.054861 & -0.009526 \\
\hline 30 & 1 & 0 & -5.693260 & 3.671773 & -0.011990 \\
\hline 31 & 1 & 0 & -5.905526 & 1.245412 & -0.003917 \\
\hline 32 & 1 & 0 & -1.432035 & 3.325357 & -0.009393 \\
\hline 33 & 6 & 0 & 4.652531 & 0.016023 & 0.758335 \\
\hline 34 & 6 & 0 & 4.621871 & -0.048698 & -0.761956 \\
\hline 35 & 6 & 0 & 4.691772 & -1.511558 & -1.238143 \\
\hline 36 & 1 & 0 & 4.138324 & -1.617383 & -2.147757 \\
\hline 37 & 1 & 0 & 5.712731 & -1.782886 & -1.408217 \\
\hline 38 & 1 & 0 & 4.272829 & -2.150809 & -0.489314 \\
\hline 39 & 6 & 0 & 5.840610 & 0.680276 & -1.357668 \\
\hline 40 & 1 & 0 & 6.739545 & 0.225421 & -0.997220 \\
\hline 41 & 1 & 0 & 5.809921 & 0.612770 & -2.425095 \\
\hline 42 & 1 & 0 & 5.819150 & 1.709133 & -1.064591 \\
\hline 43 & 6 & 0 & 5.826379 & 0.892650 & 1.232895 \\
\hline 44 & 1 & 0 & 5.802659 & 0.973539 & 2.299569 \\
\hline 45 & 1 & 0 & 6.750293 & 0.446286 & 0.929510 \\
\hline 46 & 1 & 0 & 5.741780 & 1.867209 & 0.799331 \\
\hline 47 & 6 & 0 & 4.864989 & -1.387259 & 1.356048 \\
\hline 48 & 1 & 0 & 5.803281 & -1.778703 & 1.022460 \\
\hline 49 & 1 & 0 & 4.864475 & -1.323800 & 2.424164 \\
\hline 50 & 1 & 0 & 4.074830 & -2.034281 & 1.036812 \\
\hline
\end{tabular}

pyrene (1i) ( $D_{2 \mathrm{~h}}$ symmetry)

$\mathrm{E}(\mathrm{B} 3 \mathrm{LYP} / 6-31 \mathrm{G}(\mathrm{d}))=-615.77313432$ hartree

\begin{tabular}{rrrrrr} 
Center & Atomic & Atomic & \multicolumn{3}{c}{ Coordinates (Angstroms) } \\
Number & Number & Type & X & Y & $Z$ \\
\hline 1 & 6 & 0 & 3.515933 & -0.000074 & 0.000184 \\
2 & 6 & 0 & 2.827166 & -1.210704 & 0.000184 \\
3 & 6 & 0 & 1.426443 & -1.228883 & 0.000184 \\
4 & 6 & 0 & 0.716656 & -0.000013 & 0.000146 \\
5 & 6 & 0 & 1.426537 & 1.228847 & 0.000116 \\
6 & 6 & 0 & 2.827195 & 1.210647 & 0.000219 \\
7 & 6 & 0 & 0.678249 & -2.458865 & 0.000119 \\
8 & 6 & 0 & -0.716664 & -0.000018 & -0.000073 \\
9 & 6 & 0 & -1.426511 & -1.228845 & -0.000108 \\
10 & 6 & 0 & -0.678351 & -2.458844 & 0.000030 \\
11 & 6 & 0 & -2.827247 & -1.210638 & -0.000265 \\
12 & 1 & 0 & -3.382475 & -2.160525 & -0.000233 \\
13 & 6 & 0 & -3.515937 & 0.000029 & -0.000416 \\
14 & 6 & 0 & -2.827137 & 1.210717 & -0.000443 \\
& & 0 & &
\end{tabular}




$\begin{array}{rrrrrr}15 & 6 & 0 & -1.426474 & 1.228888 & -0.000235 \\ 16 & 6 & 0 & -0.678224 & 2.458875 & -0.000025 \\ 17 & 6 & 0 & 0.678358 & 2.458855 & 0.000130 \\ 18 & 1 & 0 & 1.246976 & 3.401570 & 0.000653 \\ 19 & 1 & 0 & -1.246741 & 3.401654 & -0.000107 \\ 20 & 1 & 0 & 1.246882 & -3.401544 & 0.000415 \\ 21 & 1 & 0 & 4.616403 & -0.000036 & 0.000447 \\ 22 & 1 & 0 & 3.382372 & -2.160574 & 0.000278 \\ 23 & 1 & 0 & 3.382379 & 2.160532 & 0.000599 \\ 24 & 1 & 0 & -1.246864 & -3.401596 & 0.000317 \\ 25 & 1 & 0 & -4.616434 & 0.000114 & -0.000391 \\ 26 & 1 & 0 & -3.382446 & 2.160559 & -0.000461\end{array}$

diiodo-(pyrene-1-yl)borane (pyrene-1-BI 2$)\left(C_{1}\right.$ symmetry)

$\mathrm{E}(\mathrm{B} 3 \mathrm{LYP} / 631 \mathrm{LAN})=-662.84763312$ hartree

\begin{tabular}{|c|c|c|c|c|c|}
\hline \multirow{2}{*}{$\begin{array}{l}\text { Center } \\
\text { Number }\end{array}$} & \multirow{2}{*}{$\begin{array}{l}\text { Atomic } \\
\text { Number }\end{array}$} & \multirow{2}{*}{$\begin{array}{c}\text { Atomic } \\
\text { Type }\end{array}$} & \multicolumn{3}{|c|}{ Coordinates (Angstroms) } \\
\hline & & & $\mathrm{X}$ & Y & $\mathrm{Z}$ \\
\hline 1 & 5 & 0 & 1.711635 & -0.000263 & 0.247172 \\
\hline 2 & 53 & 0 & 2.728399 & 1.914133 & -0.183385 \\
\hline 3 & 53 & 0 & 2.728289 & -1.913696 & -0.187900 \\
\hline 4 & 6 & 0 & -5.619360 & 0.002595 & -1.919282 \\
\hline 5 & 6 & 0 & -5.788900 & 0.000819 & -0.536792 \\
\hline 6 & 6 & 0 & -4.675637 & -0.000390 & 0.313501 \\
\hline 7 & 6 & 0 & -3.372845 & 0.000253 & -0.249217 \\
\hline 8 & 6 & 0 & -3.209372 & 0.002094 & -1.658934 \\
\hline 9 & 6 & 0 & -4.344288 & 0.003164 & -2.479993 \\
\hline 10 & 6 & 0 & -4.809032 & -0.002170 & 1.746977 \\
\hline 11 & 6 & 0 & -2.222579 & -0.000747 & 0.605940 \\
\hline 12 & 6 & 0 & -2.386059 & -0.002523 & 2.015609 \\
\hline 13 & 6 & 0 & -3.720320 & -0.003234 & 2.556339 \\
\hline 14 & 6 & 0 & -1.251076 & -0.003540 & 2.836710 \\
\hline 15 & 1 & 0 & -1.372219 & -0.004977 & 3.930275 \\
\hline 16 & 6 & 0 & 0.023925 & -0.002784 & 2.276014 \\
\hline 17 & 6 & 0 & 0.193473 & -0.000982 & 0.893457 \\
\hline 18 & 6 & 0 & -0.919746 & 0.000019 & 0.043206 \\
\hline 19 & 6 & 0 & -0.786393 & 0.001654 & -1.390307 \\
\hline 20 & 6 & 0 & -1.875091 & 0.002653 & -2.199659 \\
\hline 21 & 1 & 0 & -1.768973 & 0.003540 & -3.295458 \\
\hline 22 & 1 & 0 & 0.232338 & 0.002179 & -1.807716 \\
\hline 23 & 1 & 0 & -5.827797 & -0.002908 & 2.164236 \\
\hline 24 & 1 & 0 & -6.502488 & 0.003268 & -2.575878 \\
\hline 25 & 1 & 0 & -6.801181 & 0.000264 & -0.105751 \\
\hline 26 & 1 & 0 & -4.223112 & 0.004190 & -3.573531 \\
\hline 27 & 1 & 0 & -3.826544 & -0.004931 & 3.652105 \\
\hline 28 & 1 & 0 & 0.907147 & -0.003745 & 2.932527 \\
\hline
\end{tabular}

diiodo-(pyrene-2-yl)borane (pyrene-2-BI $)\left(C_{1}\right.$ symmetry)

$\mathrm{E}(\mathrm{B} 3 \mathrm{LYP} / 631 \mathrm{LAN})=-662.85528307$ hartree

$\begin{array}{llll}\text { Center Atomic } & \text { Atomic } & \text { Coordinates (Angstroms) }\end{array}$ 


\begin{tabular}{|c|c|c|c|c|c|}
\hline Number & Number & Type & $X$ & $\mathrm{Y}$ & Z \\
\hline 1 & 5 & 0 & -1.990323 & -0.000039 & 0.000035 \\
\hline 2 & 53 & 0 & -3.095520 & -1.913815 & 0.010028 \\
\hline 3 & 53 & 0 & -3.095127 & 1.913964 & -0.010053 \\
\hline 4 & 6 & 0 & 6.691547 & -0.000171 & -0.000036 \\
\hline 5 & 6 & 0 & 6.002822 & 0.008630 & 1.210586 \\
\hline 6 & 6 & 0 & 4.602099 & 0.008874 & 1.228813 \\
\hline 7 & 6 & 0 & 3.892270 & 0.000015 & -0.000001 \\
\hline 8 & 6 & 0 & 4.602108 & -0.008949 & -1.228853 \\
\hline 9 & 6 & 0 & 6.002766 & -0.008827 & -1.210702 \\
\hline 10 & 6 & 0 & 3.853948 & 0.017754 & 2.458789 \\
\hline 11 & 6 & 0 & 2.458950 & -0.000089 & 0.000055 \\
\hline 12 & 6 & 0 & 1.749146 & 0.008810 & 1.228874 \\
\hline 13 & 6 & 0 & 2.497349 & 0.017774 & 2.458815 \\
\hline 14 & 6 & 0 & 0.348409 & 0.008634 & 1.210716 \\
\hline 15 & 1 & 0 & -0.206786 & 0.015573 & 2.160598 \\
\hline 16 & 6 & 0 & -0.340323 & -0.000208 & 0.000106 \\
\hline 17 & 6 & 0 & 0.348435 & -0.009036 & -1.210574 \\
\hline 18 & 6 & 0 & 1.749097 & -0.009072 & -1.228794 \\
\hline 19 & 6 & 0 & 2.497305 & -0.017808 & -2.458776 \\
\hline 20 & 6 & 0 & 3.853886 & -0.017761 & -2.458803 \\
\hline 21 & 1 & 0 & 4.422471 & -0.024094 & -3.401516 \\
\hline 22 & 1 & 0 & 1.928755 & -0.024655 & -3.401510 \\
\hline 23 & 1 & 0 & 4.422614 & 0.024815 & 3.401422 \\
\hline 24 & 1 & 0 & 7.792016 & 0.000003 & -0.000113 \\
\hline 25 & 1 & 0 & 6.558061 & 0.015542 & 2.160412 \\
\hline 26 & 1 & 0 & 6.557918 & -0.015354 & -2.160583 \\
\hline 27 & 1 & 0 & 1.928868 & 0.024917 & 3.401559 \\
\hline 28 & 1 & 0 & -0.206907 & -0.015871 & -2.160372 \\
\hline
\end{tabular}

diiodo-(pyrene-4-yl)borane (pyrene-4-BI $)$ ( $C_{1}$ symmetry)

$\mathrm{E}(\mathrm{B} 3 \mathrm{LYP} / 631 \mathrm{LAN})=-662.84697181$ hartree

\begin{tabular}{rrrrrr} 
Center & Atomic & Atomic & \multicolumn{2}{c}{ Coordinates (Angstroms) } \\
Number & Number & Type & $\mathrm{X}$ & $\mathrm{Y}$ & $\mathrm{Z}$ \\
& & & & & \\
\hline & 5 & 0 & -1.490584 & -0.231786 & -0.000076 \\
3 & 53 & 0 & -2.593138 & -0.152177 & -1.913747 \\
4 & 53 & 0 & -2.592344 & -0.152955 & 1.914085 \\
5 & 6 & 0 & 4.249215 & -2.972360 & -0.000817 \\
6 & 6 & 0 & 2.857864 & -2.907800 & -0.000882 \\
7 & 6 & 0 & 2.206177 & -1.667776 & -0.000554 \\
8 & 6 & 0 & 2.979281 & -0.477722 & -0.000191 \\
9 & 6 & 0 & 4.396457 & -0.552826 & -0.000158 \\
10 & 6 & 0 & 5.015694 & -1.809297 & -0.000390 \\
11 & 6 & 0 & 0.770619 & -1.559038 & -0.000674 \\
12 & 6 & 0 & 2.329001 & 0.799597 & -0.000070 \\
13 & 6 & 0 & 0.911870 & 0.874686 & -0.000169 \\
14 & 6 & 0 & 0.155170 & -0.350078 & -0.000441 \\
15 & 6 & 0 & 0.292603 & 2.131230 & 0.000009 \\
16 & 1 & 0 & -0.805799 & 2.195078 & -0.000006 \\
17 & 6 & 0 & 1.059056 & 3.294225 & 0.000250 \\
& 6 & 0 & 2.450474 & 3.229662 & 0.000289 \\
& & & & &
\end{tabular}




$\begin{array}{rrrrrr}18 & 6 & 0 & 3.102126 & 1.989689 & 0.000168 \\ 19 & 6 & 0 & 4.537714 & 1.880902 & 0.000433 \\ 20 & 6 & 0 & 5.153156 & 0.671959 & 0.000266 \\ 21 & 1 & 0 & 6.251240 & 0.592922 & 0.000832 \\ 22 & 1 & 0 & 5.119955 & 2.815267 & 0.000664 \\ 23 & 1 & 0 & 0.188519 & -2.493461 & -0.000690 \\ 24 & 1 & 0 & 4.748514 & -3.953040 & -0.000815 \\ 25 & 1 & 0 & 2.263264 & -3.833521 & -0.001099 \\ 26 & 1 & 0 & 6.114074 & -1.873108 & 0.000038 \\ 27 & 1 & 0 & 0.559853 & 4.274985 & 0.000536 \\ 28 & 1 & 0 & 3.045002 & 4.155461 & 0.000582\end{array}$

\section{2,2'-(2,7-pyrenediyl)bis [4,4,5,5-tetramethyl-1,3,2-dioxaborolane]}

(pyrene-2,7-(Bpin) $\left.)_{2}\right)\left(C_{1}\right.$ symmetry)

$\mathrm{E}(\mathrm{B} 3 \mathrm{LYP} / 6-31 \mathrm{G}(\mathrm{d}))=-1437.15846474$ hartree

\begin{tabular}{|c|c|c|c|c|c|}
\hline \multirow{2}{*}{$\begin{array}{l}\text { Center } \\
\text { Number }\end{array}$} & \multirow{2}{*}{$\begin{array}{l}\text { Atomic } \\
\text { Number }\end{array}$} & \multirow{2}{*}{$\begin{array}{c}\text { Atomic } \\
\text { Type }\end{array}$} & \multicolumn{3}{|c|}{ Coordinates (Angstroms) } \\
\hline & & & $X$ & $\mathrm{Y}$ & $\mathrm{Z}$ \\
\hline 1 & 5 & 0 & 4.889836 & -1.316304 & -0.025092 \\
\hline 2 & 5 & 0 & -4.889836 & 1.316527 & -0.008278 \\
\hline 3 & 8 & 0 & -5.586867 & 0.773299 & 1.231076 \\
\hline 4 & 8 & 0 & -5.596795 & 0.749172 & -1.230722 \\
\hline 5 & 8 & 0 & 5.586708 & -0.789557 & 1.221445 \\
\hline 6 & 8 & 0 & 5.596953 & -0.732794 & -1.239815 \\
\hline 7 & 6 & 0 & -3.402783 & 0.916192 & -0.010839 \\
\hline 8 & 6 & 0 & -2.420868 & 1.905572 & -0.011313 \\
\hline 9 & 6 & 0 & -1.058586 & 1.565374 & -0.013633 \\
\hline 10 & 6 & 0 & -0.688777 & 0.185542 & -0.015510 \\
\hline 11 & 6 & 0 & -1.701341 & -0.822136 & -0.015011 \\
\hline 12 & 6 & 0 & -3.050293 & -0.432430 & -0.012665 \\
\hline 13 & 6 & 0 & -0.016884 & 2.556243 & -0.014192 \\
\hline 14 & 6 & 0 & 0.688777 & -0.185316 & -0.017877 \\
\hline 15 & 6 & 0 & 1.701340 & 0.822361 & -0.018377 \\
\hline 16 & 6 & 0 & 1.297893 & 2.202286 & -0.016453 \\
\hline 17 & 6 & 0 & 3.050294 & 0.432655 & -0.020720 \\
\hline 18 & 1 & 0 & 3.822648 & 1.198070 & -0.021104 \\
\hline 19 & 6 & 0 & 3.402784 & -0.915965 & -0.022537 \\
\hline 20 & 6 & 0 & 2.420868 & -1.905346 & -0.022065 \\
\hline 21 & 6 & 0 & 1.058588 & -1.565150 & -0.019751 \\
\hline 22 & 6 & 0 & 0.016884 & -2.556019 & -0.019195 \\
\hline 23 & 6 & 0 & -1.297892 & -2.202062 & -0.016938 \\
\hline 24 & 1 & 0 & -2.072539 & -2.965282 & -0.016555 \\
\hline 25 & 1 & 0 & 0.303662 & -3.604990 & -0.020628 \\
\hline 26 & 1 & 0 & -0.303659 & 3.605215 & -0.012760 \\
\hline 27 & 1 & 0 & -2.704561 & 2.955289 & -0.009878 \\
\hline 28 & 1 & 0 & -3.822651 & -1.197841 & -0.012282 \\
\hline 29 & 1 & 0 & 2.072541 & 2.965506 & -0.016848 \\
\hline 30 & 1 & 0 & 2.704563 & -2.955063 & -0.023494 \\
\hline 31 & 6 & 0 & 6.791463 & 0.070483 & -0.745898 \\
\hline 32 & 6 & 0 & 6.744688 & 0.091663 & 0.775213 \\
\hline 33 & 6 & 0 & -6.791365 & -0.060585 & -0.747652 \\
\hline 34 & 6 & 0 & -6.744787 & -0.101929 & 0.773051 \\
\hline 35 & 6 & 0 & -6.550014 & -1.548295 & 1.264671 \\
\hline & & & S58 & & \\
\hline
\end{tabular}




$\begin{array}{rrrrrr}36 & 1 & 0 & -5.944126 & -1.546693 & 2.146599 \\ 37 & 1 & 0 & -7.503070 & -1.980659 & 1.487482 \\ 38 & 1 & 0 & -6.067516 & -2.122475 & 0.501511 \\ 39 & 6 & 0 & -8.072495 & 0.403563 & 1.367414 \\ 40 & 1 & 0 & -8.877163 & -0.205763 & 1.012263 \\ 41 & 1 & 0 & -8.028704 & 0.349709 & 2.435161 \\ 42 & 1 & 0 & -8.234116 & 1.417960 & 1.067786 \\ 43 & 6 & 0 & -8.104751 & 0.586349 & -1.225242 \\ 44 & 1 & 0 & -8.100278 & 0.656242 & -2.292947 \\ 45 & 1 & 0 & -8.932928 & -0.013647 & -0.910555 \\ 46 & 1 & 0 & -8.193596 & 1.565946 & -0.804056 \\ 47 & 6 & 0 & -6.750922 & -1.485762 & -1.329748 \\ 48 & 1 & 0 & -7.612061 & -2.029580 & -1.001721 \\ 49 & 1 & 0 & -6.747217 & -1.434534 & -2.398515 \\ 50 & 1 & 0 & -5.865388 & -1.983391 & -0.993452 \\ 51 & 6 & 0 & 6.549855 & 1.531384 & 1.285940 \\ 52 & 1 & 0 & 5.943246 & 1.518157 & 2.167275 \\ 53 & 1 & 0 & 7.502830 & 1.960500 & 1.515280 \\ 54 & 1 & 0 & 6.068116 & 2.115820 & 0.530121 \\ 55 & 6 & 0 & 6.751098 & 1.503252 & -1.309053 \\ 56 & 1 & 0 & 7.608539 & 2.044849 & -0.967928 \\ 57 & 1 & 0 & 6.754726 & 1.466398 & -2.378412 \\ 58 & 1 & 0 & 5.861981 & 1.994003 & -0.972103 \\ 59 & 6 & 0 & 8.072319 & -0.421670 & 1.362994 \\ 60 & 1 & 0 & 8.872725 & 0.212433 & 1.043364 \\ 61 & 1 & 0 & 8.016010 & -0.413851 & 2.431483 \\ 62 & 1 & 0 & 8.250666 & -1.420257 & 1.022529 \\ 63 & 6 & 0 & 8.104910 & -0.570067 & -1.231854 \\ 64 & 1 & 0 & 8.104944 & -0.616494 & -2.300846 \\ 65 & 1 & 0 & 8.933568 & 0.020189 & -0.900459 \\ 66 & 1 & 0 & 8.188807 & -1.558955 & -0.831900 \\ ------------------------------------ \\ \end{array}$

perylene $(\mathbf{1} \mathbf{j})\left(D_{2 \mathrm{~h}}\right.$ symmetry)

$\mathrm{E}(\mathrm{B} 3 \mathrm{LYP} / 6-31 \mathrm{G}(\mathrm{d}))=-769.40611852$ hartree

\begin{tabular}{|c|c|c|c|c|c|}
\hline \multirow{2}{*}{$\begin{array}{l}\text { Center } \\
\text { Number }\end{array}$} & \multirow{2}{*}{$\begin{array}{l}\text { Atomic } \\
\text { Number }\end{array}$} & \multirow{2}{*}{$\begin{array}{c}\text { Atomic } \\
\text { Type }\end{array}$} & \multicolumn{3}{|c|}{ Coordinates (Angstroms) } \\
\hline & & & $\mathrm{X}$ & $\mathrm{Y}$ & $\mathrm{Z}$ \\
\hline 1 & 6 & 0 & 0.000000 & 1.249831 & 0.738028 \\
\hline 2 & 6 & 0 & 0.000000 & 1.249831 & -0.738028 \\
\hline 3 & 6 & 0 & 0.000000 & 0.000000 & 1.439858 \\
\hline 4 & 6 & 0 & 0.000000 & 0.000000 & -1.439858 \\
\hline 5 & 6 & 0 & 0.000000 & -1.249831 & -0.738028 \\
\hline 6 & 6 & 0 & 0.000000 & -1.249831 & 0.738028 \\
\hline 7 & 6 & 0 & 0.000000 & -2.422655 & -2.886032 \\
\hline 8 & 6 & 0 & 0.000000 & -1.232675 & -3.575889 \\
\hline 9 & 6 & 0 & 0.000000 & 0.000000 & -2.874720 \\
\hline 10 & 6 & 0 & 0.000000 & 1.232675 & -3.575889 \\
\hline 11 & 6 & 0 & 0.000000 & 2.422655 & -2.886032 \\
\hline 12 & 1 & 0 & 0.000000 & 3.368684 & -3.420708 \\
\hline 13 & 1 & 0 & 0.000000 & 1.217784 & -4.662831 \\
\hline 14 & 1 & 0 & 0.000000 & -3.368684 & -3.420708 \\
\hline 15 & 1 & 0 & 0.000000 & -1.217784 & -4.662831 \\
\hline 16 & 6 & 0 & 0.000000 & 2.422655 & 2.886032 \\
\hline \multirow[t]{2}{*}{17} & 6 & 0 & 0.000000 & 1.232675 & 3.575889 \\
\hline & & & S59 & & \\
\hline
\end{tabular}




\begin{tabular}{rrrrrr}
18 & 6 & 0 & 0.000000 & 0.000000 & 2.874720 \\
19 & 1 & 0 & 0.000000 & 3.368684 & 3.420708 \\
20 & 1 & 0 & 0.000000 & 1.217784 & 4.662831 \\
21 & 6 & 0 & 0.000000 & -1.232675 & 3.575889 \\
22 & 6 & 0 & 0.000000 & -2.422655 & 2.886032 \\
23 & 1 & 0 & 0.000000 & -1.217784 & 4.662831 \\
24 & 1 & 0 & 0.000000 & -3.368684 & 3.420708 \\
25 & 6 & 0 & 0.000000 & 2.427304 & -1.479540 \\
26 & 1 & 0 & 0.000000 & 3.388218 & -0.977415 \\
27 & 6 & 0 & 0.000000 & -2.427304 & -1.479540 \\
28 & 1 & 0 & 0.000000 & -3.388218 & -0.977415 \\
29 & 6 & 0 & 0.000000 & 2.427304 & 1.479540 \\
30 & 1 & 0 & 0.000000 & 3.388218 & 0.977415 \\
31 & 6 & 0 & 0.000000 & -2.427304 & 1.479540 \\
32 & 1 & 0 & 0.000000 & -3.388218 & 0.977415 \\
\hline------------------------------------------------------------------------------------------------
\end{tabular}

diiodo-(perylen-1-yl)borane (perylene-1-BI $)$ ( $C_{1}$ symmetry)

$\mathrm{E}(\mathrm{B} 3 \mathrm{LYP} / 631 \mathrm{LAN})=-816.47277762$ hartree

\begin{tabular}{|c|c|c|c|c|c|}
\hline \multirow{2}{*}{$\begin{array}{l}\text { Center } \\
\text { Number }\end{array}$} & \multirow{2}{*}{$\begin{array}{l}\text { Atomic } \\
\text { Number }\end{array}$} & \multirow{2}{*}{$\begin{array}{l}\text { Atomic } \\
\text { Type }\end{array}$} & \multicolumn{3}{|c|}{ Coordinates (Angstroms) } \\
\hline & & & $X$ & $\mathrm{Y}$ & Z \\
\hline 1 & 5 & 0 & 1.724884 & 0.511547 & 0.000117 \\
\hline 2 & 53 & 0 & 2.671056 & -0.058885 & 1.914136 \\
\hline 3 & 53 & 0 & 2.671578 & -0.058705 & -1.913697 \\
\hline 4 & 6 & 0 & -0.908709 & 0.694618 & -0.000138 \\
\hline 5 & 6 & 0 & -0.998429 & -0.778708 & -0.000195 \\
\hline 6 & 6 & 0 & -2.113569 & 1.471121 & -0.000191 \\
\hline 7 & 6 & 0 & -2.288609 & -1.403271 & -0.000171 \\
\hline 8 & 6 & 0 & -3.493470 & -0.626768 & -0.000139 \\
\hline 9 & 6 & 0 & -3.403749 & 0.846557 & -0.000191 \\
\hline 10 & 6 & 0 & -4.794689 & -2.699512 & -0.000070 \\
\hline 11 & 6 & 0 & -3.648842 & -3.460426 & -0.000135 \\
\hline 12 & 6 & 0 & -2.375826 & -2.835480 & -0.000188 \\
\hline 13 & 6 & 0 & -1.188051 & -3.610279 & -0.000257 \\
\hline 14 & 6 & 0 & 0.041661 & -2.994030 & -0.000310 \\
\hline 15 & 1 & 0 & 0.953442 & -3.585220 & -0.000374 \\
\hline 16 & 1 & 0 & -1.268983 & -4.694306 & -0.000278 \\
\hline 17 & 1 & 0 & -5.771469 & -3.175695 & -0.000016 \\
\hline 18 & 1 & 0 & -3.700047 & -4.546262 & -0.000148 \\
\hline 19 & 6 & 0 & 0.392511 & 2.767362 & -0.000048 \\
\hline 20 & 6 & 0 & -0.753338 & 3.528276 & -0.000155 \\
\hline 21 & 6 & 0 & -2.026353 & 2.903329 & -0.000216 \\
\hline 22 & 1 & 0 & 1.369290 & 3.243547 & 0.000034 \\
\hline 23 & 1 & 0 & -0.702132 & 4.614112 & -0.000185 \\
\hline 24 & 6 & 0 & -3.214128 & 3.678129 & -0.000282 \\
\hline 25 & 6 & 0 & -4.443840 & 3.061879 & -0.000290 \\
\hline 26 & 1 & 0 & -3.133196 & 4.762156 & -0.000326 \\
\hline 27 & 1 & 0 & -5.355621 & 3.653069 & -0.000331 \\
\hline 28 & 6 & 0 & 0.131794 & -1.590421 & -0.000281 \\
\hline 29 & 1 & 0 & 1.121453 & -1.147632 & -0.000336 \\
\hline 30 & 6 & 0 & -4.713837 & -1.295338 & -0.000077 \\
\hline 31 & 1 & 0 & -5.642453 & -0.735733 & -0.000032 \\
\hline 32 & 6 & 0 & 0.311659 & 1.363188 & -0.000035 \\
\hline 33 & 6 & 0 & -4.533972 & 1.658270 & -0.000236 \\
\hline & & & S60 & & \\
\hline
\end{tabular}


diiodo-(perylen-2-yl)borane (perylene-2-BI $)\left(C_{1}\right.$ symmetry)

$\mathrm{E}(\mathrm{B} 3 \mathrm{LYP} / 631 \mathrm{LAN})=-816.48850286$ hartree

\begin{tabular}{|c|c|c|c|c|c|}
\hline \multirow{2}{*}{$\begin{array}{l}\text { Center } \\
\text { Number }\end{array}$} & \multirow{2}{*}{$\begin{array}{l}\text { Atomic } \\
\text { Number }\end{array}$} & \multirow{2}{*}{$\begin{array}{c}\text { Atomic } \\
\text { Type }\end{array}$} & \multicolumn{3}{|c|}{ Coordinates (Angstroms) } \\
\hline & & & $X$ & $\mathrm{Y}$ & $\mathrm{Z}$ \\
\hline 1 & 5 & 0 & 2.446844 & 0.031451 & -0.000278 \\
\hline 2 & 53 & 0 & 3.505771 & -0.289448 & -1.913338 \\
\hline 3 & 53 & 0 & 3.508201 & -0.270941 & 1.914449 \\
\hline 4 & 6 & 0 & -2.553593 & -1.185617 & 0.003514 \\
\hline 5 & 6 & 0 & -1.494391 & -0.157599 & 0.000507 \\
\hline 6 & 6 & 0 & -3.927685 & -0.777553 & 0.002559 \\
\hline 7 & 6 & 0 & -1.861230 & 1.228063 & -0.003439 \\
\hline 8 & 6 & 0 & -3.235322 & 1.636127 & -0.004480 \\
\hline 9 & 6 & 0 & -4.294523 & 0.608109 & -0.001364 \\
\hline 10 & 6 & 0 & -2.510768 & 3.973735 & -0.011322 \\
\hline 11 & 6 & 0 & -1.186952 & 3.600281 & -0.010342 \\
\hline 12 & 6 & 0 & -0.831588 & 2.227391 & -0.006401 \\
\hline 13 & 6 & 0 & 0.530080 & 1.831179 & -0.005356 \\
\hline 14 & 6 & 0 & 0.863827 & 0.496805 & -0.001523 \\
\hline 15 & 1 & 0 & 1.299687 & 2.598879 & -0.007621 \\
\hline 16 & 1 & 0 & -2.785968 & 5.024975 & -0.014351 \\
\hline 17 & 1 & 0 & -0.396603 & 4.346610 & -0.012555 \\
\hline 18 & 6 & 0 & -3.278148 & -3.523225 & 0.010197 \\
\hline 19 & 6 & 0 & -4.601963 & -3.149770 & 0.009389 \\
\hline 20 & 6 & 0 & -4.957327 & -1.776881 & 0.005563 \\
\hline 21 & 1 & 0 & -3.002949 & -4.574466 & 0.013091 \\
\hline 22 & 1 & 0 & -5.392312 & -3.896100 & 0.011645 \\
\hline 23 & 6 & 0 & -6.318995 & -1.380667 & 0.004652 \\
\hline 24 & 6 & 0 & -6.652741 & -0.046293 & 0.000881 \\
\hline 25 & 1 & 0 & -7.088602 & -2.148367 & 0.006982 \\
\hline 26 & 1 & 0 & -7.695296 & 0.260184 & 0.000165 \\
\hline 27 & 6 & 0 & -0.142220 & -0.486101 & 0.001377 \\
\hline 28 & 1 & 0 & 0.166705 & -1.525352 & 0.004369 \\
\hline 29 & 6 & 0 & -3.523290 & 2.997501 & -0.008404 \\
\hline 30 & 1 & 0 & -4.552854 & 3.337329 & -0.009302 \\
\hline 31 & 6 & 0 & -2.265626 & -2.546992 & 0.007274 \\
\hline 32 & 1 & 0 & -1.236061 & -2.886820 & 0.008027 \\
\hline 33 & 6 & 0 & -5.646694 & 0.936613 & -0.002102 \\
\hline 34 & 1 & 0 & -5.955618 & 1.975864 & -0.005048 \\
\hline
\end{tabular}

diiodo-(perylen-3-yl)borane (perylene-3-BI $\left.{ }_{2}\right)\left(C_{1}\right.$ symmetry)

$\mathrm{E}(\mathrm{B} 3 \mathrm{LYP} / 631 \mathrm{LAN})=-816.48099395$ hartree

\begin{tabular}{|c|c|c|c|c|c|}
\hline Center & Atomic & Atomic & \multicolumn{3}{|c|}{ Coordinates (Angstroms) } \\
\hline Number & Number & Type & $\mathrm{X}$ & $\mathrm{Y}$ & Z \\
\hline $\begin{array}{l}1 \\
2\end{array}$ & $\begin{array}{r}5 \\
53\end{array}$ & $\begin{array}{l}0 \\
0\end{array}$ & $\begin{array}{l}2.440890 \\
3.529511\end{array}$ & $\begin{array}{l}-0.274886 \\
-0.102952\end{array}$ & $\begin{array}{r}0.002764 \\
-1.912816\end{array}$ \\
\hline & & & S61 & & \\
\hline
\end{tabular}




$\begin{array}{rrrrrr}3 & 53 & 0 & 3.527967 & -0.058967 & 1.914763 \\ 4 & 6 & 0 & -2.386048 & 1.425417 & -0.013862 \\ 5 & 6 & 0 & -3.842605 & 1.186301 & -0.011545 \\ 6 & 6 & 0 & -1.491011 & 0.305847 & -0.003052 \\ 7 & 6 & 0 & -4.332687 & -0.160658 & 0.001336 \\ 8 & 6 & 0 & -3.437650 & -1.280228 & 0.012061 \\ 9 & 6 & 0 & -1.981094 & -1.041112 & 0.009853 \\ 10 & 6 & 0 & -5.367278 & -2.785476 & 0.026382 \\ 11 & 6 & 0 & -6.240804 & -1.723023 & 0.016239 \\ 12 & 6 & 0 & -5.748595 & -0.393100 & 0.003550 \\ 13 & 6 & 0 & -6.640200 & 0.709648 & -0.006976 \\ 14 & 6 & 0 & -6.152237 & 1.995610 & -0.019243 \\ 15 & 1 & 0 & -6.833110 & 2.842484 & -0.027316 \\ 16 & 1 & 0 & -7.710371 & 0.518874 & -0.005150 \\ 17 & 1 & 0 & -5.741630 & -3.805582 & 0.036102 \\ 18 & 1 & 0 & -7.315799 & -1.884410 & 0.017775 \\ 19 & 6 & 0 & -0.456420 & 2.930662 & -0.028342 \\ 20 & 6 & 0 & 0.417105 & 1.868211 & -0.018027 \\ 21 & 6 & 0 & -0.075104 & 0.538289 & -0.005224 \\ 22 & 1 & 0 & -0.082067 & 3.950766 & -0.038198 \\ 23 & 1 & 0 & 1.492101 & 2.029599 & -0.019521 \\ 24 & 6 & 0 & 0.816501 & -0.564458 & 0.005436 \\ 25 & 6 & 0 & 0.328538 & -1.850419 & 0.017766 \\ 26 & 1 & 0 & 1.009410 & -2.697293 & 0.025942 \\ 27 & 6 & 0 & -4.765077 & 2.228044 & -0.021470 \\ 28 & 1 & 0 & -4.425257 & 3.257564 & -0.031280 \\ 29 & 6 & 0 & -3.978612 & -2.562216 & 0.024280 \\ 30 & 1 & 0 & -3.327448 & -3.429051 & 0.032562 \\ 31 & 6 & 0 & -1.845086 & 2.707403 & -0.026246 \\ 32 & 1 & 0 & -2.496250 & 3.574236 & -0.034673 \\ 33 & 6 & 0 & -1.058622 & -2.082854 & 0.019909 \\ 34 & 1 & 0 & -1.398442 & -3.112374 & 0.029765 \\ -------------------------------------------------------------------------------------------\end{array}$

\section{$2,2^{\prime}, 2^{\prime \prime}, 2^{\prime \prime \prime}-(2,5,8,11-p e r l y e n e t e t r a y l) t e t r a k i s[4,4,5,5$-tetramethyl-1,3,2-dioxaborolane]} (perylene-2,5,8,11-(Bpin) $)_{4}\left(C_{1}\right.$ symmetry)

$\mathrm{E}(\mathrm{B} 3 \mathrm{LYP} / 6-31 \mathrm{G}(\mathrm{d}))=-2412.17735009$ hartree

\begin{tabular}{|c|c|c|c|c|c|}
\hline \multirow{2}{*}{$\begin{array}{l}\text { Center } \\
\text { Number }\end{array}$} & \multirow{2}{*}{$\begin{array}{l}\text { Atomic } \\
\text { Number }\end{array}$} & \multirow{2}{*}{$\begin{array}{l}\text { Atomic } \\
\text { Type }\end{array}$} & \multicolumn{3}{|c|}{ Coordinates (Angstroms) } \\
\hline & & & $X$ & $\mathrm{Y}$ & Z \\
\hline 1 & 5 & 0 & -3.643743 & 3.763354 & 0.004706 \\
\hline 2 & 5 & 0 & 3.643771 & 3.763378 & -0.004715 \\
\hline 3 & 5 & 0 & 3.643791 & -3.763308 & -0.010391 \\
\hline 4 & 5 & 0 & -3.643729 & -3.763331 & -0.001792 \\
\hline 5 & 8 & 0 & -4.526728 & 3.875675 & 1.239480 \\
\hline 6 & 8 & 0 & -4.537180 & 3.872697 & -1.222432 \\
\hline 7 & 8 & 0 & 4.535911 & 3.871492 & 1.223475 \\
\hline 8 & 8 & 0 & 4.528059 & 3.876944 & -1.238442 \\
\hline 9 & 8 & 0 & 4.515984 & -3.885438 & 1.231104 \\
\hline 10 & 8 & 0 & 4.547893 & -3.862953 & -1.230522 \\
\hline 11 & 8 & 0 & -4.538370 & -3.870323 & 1.224675 \\
\hline 12 & 8 & 0 & -4.525500 & -3.878021 & -1.237215 \\
\hline 13 & 6 & 0 & -0.738002 & -1.249811 & -0.003170 \\
\hline 14 & 6 & 0 & 0.738053 & -1.249806 & -0.004950 \\
\hline & & & S62 & & \\
\hline
\end{tabular}




\begin{tabular}{|c|c|c|c|c|c|}
\hline 15 & 6 & 0 & -1.439834 & 0.000018 & -0.001234 \\
\hline 16 & 6 & 0 & 1.439880 & 0.000027 & -0.004837 \\
\hline 17 & 6 & 0 & 0.738047 & 1.249856 & -0.002985 \\
\hline 18 & 6 & 0 & -0.738007 & 1.249851 & -0.001097 \\
\hline 19 & 6 & 0 & 2.886047 & 2.422687 & -0.004780 \\
\hline 20 & 6 & 0 & 3.575906 & 1.232709 & -0.006535 \\
\hline 21 & 6 & 0 & 2.874741 & 0.000032 & -0.006605 \\
\hline 22 & 6 & 0 & 3.575913 & -1.232639 & -0.008405 \\
\hline 23 & 6 & 0 & 2.886059 & -2.422622 & -0.008455 \\
\hline 24 & 1 & 0 & 4.662853 & -1.217745 & -0.009725 \\
\hline 25 & 1 & 0 & 4.662847 & 1.217821 & -0.007886 \\
\hline 26 & 6 & 0 & -2.886002 & -2.422641 & -0.001535 \\
\hline 27 & 6 & 0 & -3.575861 & -1.232663 & 0.000392 \\
\hline 28 & 6 & 0 & -2.874695 & 0.000013 & 0.000577 \\
\hline 29 & 1 & 0 & -4.662802 & -1.217776 & 0.001786 \\
\hline 30 & 6 & 0 & -3.575866 & 1.232685 & 0.002511 \\
\hline 31 & 6 & 0 & -2.886012 & 2.422667 & 0.002623 \\
\hline 32 & 1 & 0 & -4.662807 & 1.217791 & 0.003895 \\
\hline 33 & 6 & 0 & 1.479567 & -2.427275 & -0.006738 \\
\hline 34 & 1 & 0 & 0.977445 & -3.388191 & -0.006835 \\
\hline 35 & 6 & 0 & 1.479555 & 2.427330 & -0.003019 \\
\hline 36 & 1 & 0 & 0.977428 & 3.388243 & -0.001663 \\
\hline 37 & 6 & 0 & -1.479511 & -2.427285 & -0.003302 \\
\hline 38 & 1 & 0 & -0.977384 & -3.388197 & -0.004804 \\
\hline 39 & 6 & 0 & -1.479521 & 2.427320 & 0.000823 \\
\hline 40 & 1 & 0 & -0.977398 & 3.388236 & 0.000965 \\
\hline 41 & 6 & 0 & -5.954899 & 4.154800 & -0.746460 \\
\hline 42 & 6 & 0 & -5.960163 & 4.088358 & 0.774058 \\
\hline 43 & 6 & 0 & 5.954131 & 4.154080 & 0.749285 \\
\hline 44 & 6 & 0 & 5.961001 & 4.089167 & -0.771292 \\
\hline 45 & 6 & 0 & 5.961421 & -4.148865 & -0.744462 \\
\hline 46 & 6 & 0 & 5.953429 & -4.094474 & 0.776523 \\
\hline 47 & 6 & 0 & -5.955622 & -4.153342 & 0.747852 \\
\hline 48 & 6 & 0 & -5.959392 & -4.089816 & -0.772794 \\
\hline 49 & 6 & 0 & -6.413573 & -5.543643 & 1.226333 \\
\hline 50 & 1 & 0 & -6.347769 & -5.593234 & 2.293156 \\
\hline 51 & 1 & 0 & -7.426311 & -5.709395 & 0.923368 \\
\hline 52 & 1 & 0 & -5.784827 & -6.294288 & 0.794928 \\
\hline 53 & 6 & 0 & -6.940357 & -3.125373 & 1.335368 \\
\hline 54 & 1 & 0 & -6.591240 & -2.804906 & 2.294700 \\
\hline 55 & 1 & 0 & -7.008321 & -2.281893 & 0.680523 \\
\hline 56 & 1 & 0 & -7.905708 & -3.575082 & 1.439090 \\
\hline 57 & 6 & 0 & -6.855904 & -2.936049 & -1.259301 \\
\hline 58 & 1 & 0 & -6.685320 & -2.767118 & -2.302020 \\
\hline 59 & 1 & 0 & -7.882971 & -3.192057 & -1.102801 \\
\hline 60 & 1 & 0 & -6.622321 & -2.047329 & -0.711108 \\
\hline 61 & 6 & 0 & -6.533116 & -5.390178 & -1.365669 \\
\hline 62 & 1 & 0 & -7.529832 & -5.537121 & -1.005301 \\
\hline 63 & 1 & 0 & -6.546587 & -5.319877 & -2.433272 \\
\hline 64 & 1 & 0 & -5.921556 & -6.217033 & -1.070366 \\
\hline 65 & 6 & 0 & -6.939054 & 3.127958 & -1.336912 \\
\hline 66 & 1 & 0 & -6.521314 & 2.703963 & -2.226081 \\
\hline 67 & 1 & 0 & -7.117158 & 2.352415 & -0.621572 \\
\hline 68 & 1 & 0 & -7.862487 & 3.614040 & -1.573334 \\
\hline 69 & 6 & 0 & -6.412385 & 5.546015 & -1.222724 \\
\hline 70 & 1 & 0 & -6.387110 & 5.583159 & -2.291780 \\
\hline 71 & 1 & 0 & -7.410388 & 5.728950 & -0.882981 \\
\hline 72 & 1 & 0 & -5.757520 & 6.292559 & -0.824321 \\
\hline 73 & 6 & 0 & -6.534473 & 5.387580 & 1.368861 \\
\hline 74 & 1 & 0 & -7.534341 & 5.530125 & 1.015526 \\
\hline
\end{tabular}




\begin{tabular}{|c|c|c|c|c|c|}
\hline 75 & 1 & 0 & -6.539890 & 5.319100 & 2.436653 \\
\hline 76 & 1 & 0 & -5.928222 & 6.216222 & 1.067675 \\
\hline 77 & 6 & 0 & -6.857149 & 2.933660 & 1.257470 \\
\hline 78 & 1 & 0 & -6.700341 & 2.774231 & 2.303842 \\
\hline 79 & 1 & 0 & -7.883289 & 3.182672 & 1.084466 \\
\hline 80 & 1 & 0 & -6.611048 & 2.041786 & 0.719981 \\
\hline 81 & 6 & 0 & 6.411105 & 5.544818 & 1.227430 \\
\hline 82 & 1 & 0 & 6.336100 & 5.597894 & 2.293478 \\
\hline 83 & 1 & 0 & 7.426831 & 5.707303 & 0.932815 \\
\hline 84 & 1 & 0 & 5.787891 & 6.295547 & 0.788216 \\
\hline 85 & 6 & 0 & 6.937667 & 3.126649 & 1.339744 \\
\hline 86 & 1 & 0 & 6.587302 & 2.808075 & 2.299252 \\
\hline 87 & 1 & 0 & 7.005931 & 2.281968 & 0.686481 \\
\hline 88 & 1 & 0 & 7.903135 & 3.576042 & 1.443754 \\
\hline 89 & 6 & 0 & 6.535932 & 5.388988 & -1.364182 \\
\hline 90 & 1 & 0 & 7.533172 & 5.534451 & -1.004667 \\
\hline 91 & 1 & 0 & 6.548333 & 5.319088 & -2.431824 \\
\hline 92 & 1 & 0 & 5.925753 & 6.216549 & -1.067998 \\
\hline 93 & 6 & 0 & 6.858504 & 2.934959 & -1.254917 \\
\hline 94 & 1 & 0 & 6.703443 & 2.777156 & -2.301796 \\
\hline 95 & 1 & 0 & 7.884413 & 3.183432 & -1.079782 \\
\hline 96 & 1 & 0 & 6.611246 & 2.042339 & -0.719200 \\
\hline 97 & 6 & 0 & 6.846126 & -2.943672 & 1.276870 \\
\hline 98 & 1 & 0 & 6.957516 & -2.215412 & 0.500899 \\
\hline 99 & 1 & 0 & 6.393468 & -2.487123 & 2.132185 \\
\hline 100 & 1 & 0 & 7.807643 & -3.328898 & 1.545169 \\
\hline 101 & 6 & 0 & 6.522580 & -5.398387 & 1.365995 \\
\hline 102 & 1 & 0 & 7.529039 & -5.532861 & 1.028570 \\
\hline 103 & 1 & 0 & 6.509072 & -5.342501 & 2.434450 \\
\hline 104 & 1 & 0 & 5.925077 & -6.225765 & 1.044535 \\
\hline 105 & 6 & 0 & 6.950648 & -3.117409 & -1.318159 \\
\hline 106 & 1 & 0 & 7.943553 & -3.361917 & -1.003110 \\
\hline 107 & 1 & 0 & 6.901714 & -3.132418 & -2.386934 \\
\hline 108 & 1 & 0 & 6.693997 & -2.141232 & -0.963041 \\
\hline 109 & 6 & 0 & 6.423091 & -5.536278 & -1.227729 \\
\hline 110 & 1 & 0 & 6.374057 & -5.576436 & -2.295850 \\
\hline 111 & 1 & 0 & 7.430446 & -5.707556 & -0.910240 \\
\hline 112 & 1 & 0 & 5.785540 & -6.288823 & -0.812874 \\
\hline
\end{tabular}

phenoxybenzene (1k) ( $C_{2}$ symmetry)

$\mathrm{E}(\mathrm{B} 3 \mathrm{LYP} / 6-31 \mathrm{G}(\mathrm{d}))=-538.51281327$ hartree

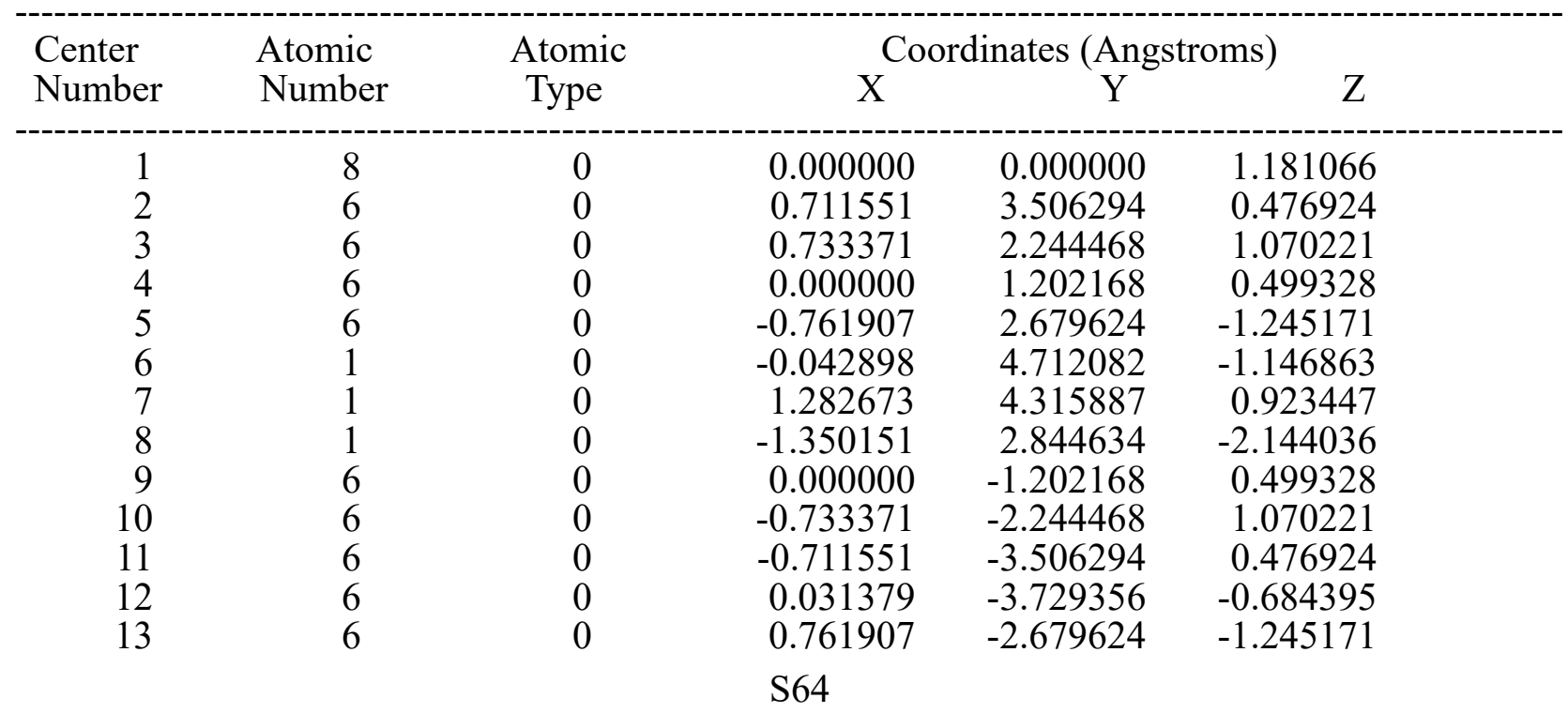




\begin{tabular}{rlrrrr}
14 & 1 & 0 & -1.305945 & -2.049854 & 1.971688 \\
15 & 1 & 0 & -1.282673 & -4.315887 & 0.923447 \\
16 & 1 & 0 & 0.042898 & -4.712082 & -1.146863 \\
17 & 1 & 0 & 1.350151 & -2.844634 & -2.144036 \\
18 & 1 & 0 & 1.305945 & 2.049854 & 1.971688 \\
19 & 6 & 0 & -0.031379 & 3.729356 & -0.684395 \\
20 & 6 & 0 & -0.756284 & 1.414161 & -0.657417 \\
21 & 1 & 0 & -1.332542 & 0.600540 & -1.085442 \\
22 & 6 & 0 & 0.756284 & -1.414161 & -0.657417 \\
23 & 1 & 0 & 1.332542 & -0.600540 & -1.085442 \\
\hline
\end{tabular}

diiodo-(2-phenoxyphenyl)borane (diphenylether-2-BI $)_{2}\left(C_{1}\right.$ symmetry)

$\mathrm{E}(\mathrm{B} 3 \mathrm{LYP} / 631 \mathrm{LAN})=-585.59026733$ hartree

\begin{tabular}{|c|c|c|c|c|c|}
\hline \multirow{2}{*}{$\begin{array}{l}\text { Center } \\
\text { Number }\end{array}$} & \multirow{2}{*}{$\begin{array}{l}\text { Atomic } \\
\text { Number }\end{array}$} & \multirow{2}{*}{$\begin{array}{l}\text { Atomic } \\
\text { Type }\end{array}$} & \multicolumn{3}{|c|}{ Coordinates (Angstroms) } \\
\hline & & & $X$ & $\mathrm{Y}$ & $\mathrm{Z}$ \\
\hline 1 & 5 & 0 & 1.199094 & 0.182562 & -0.185728 \\
\hline 2 & 53 & 0 & 2.223064 & 0.347977 & 1.765739 \\
\hline 3 & 53 & 0 & 1.532901 & -1.590530 & -1.461979 \\
\hline 4 & 8 & 0 & -1.489451 & 0.381184 & 0.644004 \\
\hline 5 & 6 & 0 & -5.186545 & -1.547616 & 0.655823 \\
\hline 6 & 6 & 0 & -4.532567 & -1.227228 & 1.847471 \\
\hline 7 & 6 & 0 & -3.310571 & -0.555757 & 1.824306 \\
\hline 8 & 6 & 0 & -2.737798 & -0.209998 & 0.598531 \\
\hline 9 & 6 & 0 & -4.603155 & -1.197967 & -0.563800 \\
\hline 10 & 1 & 0 & -6.140437 & -2.066654 & 0.677036 \\
\hline 11 & 1 & 0 & -4.975944 & -1.495670 & 2.802647 \\
\hline 12 & 1 & 0 & -5.099624 & -1.450075 & -1.497170 \\
\hline 13 & 6 & 0 & -1.141050 & 1.372963 & -0.253172 \\
\hline 14 & 6 & 0 & 0.185369 & 1.382863 & -0.689848 \\
\hline 15 & 6 & 0 & 0.624166 & 2.402936 & -1.533389 \\
\hline 16 & 6 & 0 & -0.254907 & 3.406097 & -1.947266 \\
\hline 17 & 6 & 0 & -1.578561 & 3.386375 & -1.502747 \\
\hline 18 & 1 & 0 & 1.656993 & 2.408709 & -1.871380 \\
\hline 19 & 1 & 0 & 0.088518 & 4.196853 & -2.607969 \\
\hline 20 & 1 & 0 & -2.268677 & 4.166951 & -1.812032 \\
\hline 21 & 1 & 0 & -2.787254 & -0.296211 & 2.739167 \\
\hline 22 & 6 & 0 & -3.376719 & -0.533696 & -0.602287 \\
\hline 23 & 1 & 0 & -2.916653 & -0.271598 & -1.549337 \\
\hline 24 & 6 & 0 & -2.029118 & 2.376743 & -0.651538 \\
\hline 25 & 1 & 0 & -3.054212 & 2.366468 & -0.296102 \\
\hline
\end{tabular}

diiodo-(3-phenoxyphenyl)borane (diphenylether-3-BI $)$ ( $C_{1}$ symmetry)

$\mathrm{E}(\mathrm{B} 3 \mathrm{LYP} / 631 \mathrm{LAN})=-585.59411178$ hartree

\begin{tabular}{|c|c|c|c|c|c|}
\hline \multirow{2}{*}{$\begin{array}{l}\text { Center } \\
\text { Number }\end{array}$} & \multirow{2}{*}{$\begin{array}{l}\text { Atomic } \\
\text { Number }\end{array}$} & \multirow{2}{*}{$\begin{array}{l}\text { Atomic } \\
\text { Type }\end{array}$} & \multicolumn{3}{|c|}{ Coordinates (Angstroms) } \\
\hline & & & $X$ & $\mathrm{Y}$ & $\mathrm{Z}$ \\
\hline 1 & 5 & 0 & 1.756399 & 0.006526 & 0.133511 \\
\hline 2 & 53 & 0 & 3.040884 & -1.748155 & -0.260562 \\
\hline 3 & 53 & 0 & 2.524633 & 2.044653 & -0.240594 \\
\hline 4 & 8 & 0 & -3.100446 & -0.837706 & -0.651243 \\
\hline 5 & 6 & 0 & -7.005921 & 0.603312 & -0.407017 \\
\hline 6 & 6 & 0 & -6.735849 & -0.680506 & -0.885803 \\
\hline
\end{tabular}




$\begin{array}{rrrrrr}7 & 6 & 0 & -5.425738 & -1.156013 & -0.932577 \\ 8 & 6 & 0 & -4.379782 & -0.336361 & -0.503290 \\ 9 & 6 & 0 & -5.951501 & 1.414219 & 0.018077 \\ 10 & 1 & 0 & -8.027785 & 0.969365 & -0.367509 \\ 11 & 1 & 0 & -7.547991 & -1.320113 & -1.220962 \\ 12 & 1 & 0 & -6.149878 & 2.418006 & 0.384513 \\ 13 & 6 & 0 & -2.122310 & -0.574812 & 0.289008 \\ 14 & 6 & 0 & -0.824155 & -0.394711 & -0.193205 \\ 15 & 6 & 0 & 0.223825 & -0.205097 & 0.707038 \\ 16 & 6 & 0 & -0.019152 & -0.187442 & 2.082191 \\ 17 & 6 & 0 & -1.321276 & -0.370155 & 2.552442 \\ 18 & 1 & 0 & -0.656537 & -0.410017 & -1.265525 \\ 19 & 1 & 0 & 0.798407 & -0.035025 & 2.780852 \\ 20 & 1 & 0 & -1.520010 & -0.366623 & 3.620950 \\ 21 & 1 & 0 & -5.195058 & -2.151146 & -1.299583 \\ 22 & 6 & 0 & -4.634928 & 0.954776 & -0.031242 \\ 23 & 1 & 0 & -3.813561 & 1.587840 & 0.287889 \\ 24 & 6 & 0 & -2.378179 & -0.569621 & 1.663575 \\ 25 & 1 & 0 & -3.388617 & -0.723202 & 2.027838\end{array}$

diiodo-(4-phenoxyphenyl)borane (diphenylether-4-BI $)_{2}\left(C_{1}\right.$ symmetry)

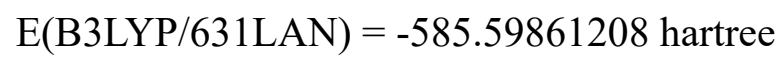

\begin{tabular}{|c|c|c|c|c|c|}
\hline \multirow{2}{*}{$\begin{array}{l}\text { Center } \\
\text { Number }\end{array}$} & \multirow{2}{*}{$\begin{array}{l}\text { Atomic } \\
\text { Number }\end{array}$} & \multirow{2}{*}{$\begin{array}{l}\text { Atomic } \\
\text { Type }\end{array}$} & \multicolumn{3}{|c|}{ Coordinates (Angstroms) } \\
\hline & & & $\mathrm{X}$ & $\mathrm{Y}$ & $\mathrm{Z}$ \\
\hline 1 & 5 & 0 & -1.855264 & -0.010489 & 0.035316 \\
\hline 2 & 53 & 0 & -3.187309 & -1.735601 & -0.330394 \\
\hline 3 & 53 & 0 & -2.680373 & 2.039670 & 0.047290 \\
\hline 4 & 8 & 0 & 3.802571 & -0.939632 & 1.032289 \\
\hline 5 & 6 & 0 & 7.033005 & 0.775100 & -0.970934 \\
\hline 6 & 6 & 0 & 7.057263 & -0.561764 & -0.567735 \\
\hline 7 & 6 & 0 & 5.955762 & -1.125975 & 0.074951 \\
\hline 8 & 6 & 0 & 4.826129 & -0.342693 & 0.321003 \\
\hline 9 & 6 & 0 & 5.898289 & 1.548750 & -0.718021 \\
\hline 10 & 1 & 0 & 7.890689 & 1.210644 & -1.475355 \\
\hline 11 & 1 & 0 & 7.935341 & -1.173276 & -0.757563 \\
\hline 12 & 1 & 0 & 5.872280 & 2.592446 & -1.020079 \\
\hline 13 & 6 & 0 & 2.480485 & -0.659073 & 0.743650 \\
\hline 14 & 6 & 0 & 1.600976 & -0.610207 & 1.827277 \\
\hline 15 & 6 & 0 & 0.239688 & -0.410733 & 1.599771 \\
\hline 16 & 6 & 0 & -0.244719 & -0.253171 & 0.299418 \\
\hline 17 & 6 & 0 & 0.644285 & -0.305444 & -0.776216 \\
\hline 18 & 1 & 0 & 1.997589 & -0.733142 & 2.830165 \\
\hline 19 & 1 & 0 & -0.442770 & -0.373643 & 2.444684 \\
\hline 20 & 1 & 0 & 0.276600 & -0.192475 & -1.792711 \\
\hline 21 & 1 & 0 & 5.954067 & -2.163280 & 0.394626 \\
\hline 22 & 6 & 0 & 4.792296 & 0.999841 & -0.068127 \\
\hline 23 & 1 & 0 & 3.914423 & 1.602792 & 0.139349 \\
\hline 24 & 6 & 0 & 2.007656 & -0.513089 & -0.564040 \\
\hline 25 & 1 & 0 & 2.697988 & -0.565433 & -1.399480 \\
\hline
\end{tabular}

\section{4,4,5,5-tetramethyl-2-(3-phenoxyphenyl)-1,3,2-dioxaborolane}

(diphenylether-3-Bpin) ( $C_{1}$ symmetry)

$\mathrm{E}(\mathrm{B} 3 \mathrm{LYP} / 6-31 \mathrm{G}(\mathrm{d}))=-\mathbf{9 4 9} .20545654$ hartree 


\begin{tabular}{|c|c|c|c|c|c|}
\hline \multirow{2}{*}{$\begin{array}{l}\text { Center } \\
\text { Number }\end{array}$} & \multirow{2}{*}{$\begin{array}{l}\text { Atomic } \\
\text { Number }\end{array}$} & \multirow{2}{*}{$\begin{array}{l}\text { Atomic } \\
\text { Type }\end{array}$} & \multicolumn{3}{|c|}{ Coordinates (Angstroms) } \\
\hline & & & $X$ & $\mathrm{Y}$ & Z \\
\hline 1 & 5 & 0 & 1.865799 & -0.498107 & -0.795692 \\
\hline 2 & 8 & 0 & 2.652567 & 0.757140 & -1.144121 \\
\hline 3 & 8 & 0 & 2.745093 & -1.373286 & 0.086266 \\
\hline 4 & 8 & 0 & -2.950869 & -1.106186 & -0.160836 \\
\hline 5 & 6 & 0 & 0.577548 & 0.806520 & 0.970864 \\
\hline 6 & 6 & 0 & 0.561442 & -0.131445 & -0.063701 \\
\hline 7 & 6 & 0 & -0.633465 & -0.739155 & -0.447850 \\
\hline 8 & 6 & 0 & -1.817893 & -0.409388 & 0.214362 \\
\hline 9 & 6 & 0 & -0.612997 & 1.127008 & 1.626583 \\
\hline 10 & 1 & 0 & 1.508990 & 1.281606 & 1.264923 \\
\hline 11 & 1 & 0 & -0.610138 & 1.849359 & 2.438629 \\
\hline 12 & 6 & 0 & -4.185384 & -0.486070 & -0.197803 \\
\hline 13 & 6 & 0 & -5.284718 & -1.259147 & 0.181652 \\
\hline 14 & 6 & 0 & -6.567982 & -0.720790 & 0.091884 \\
\hline 15 & 6 & 0 & -6.757125 & 0.584619 & -0.366948 \\
\hline 16 & 6 & 0 & -5.650187 & 1.347416 & -0.744940 \\
\hline 17 & 1 & 0 & -5.116364 & -2.270797 & 0.537211 \\
\hline 18 & 1 & 0 & -7.421887 & -1.324611 & 0.387263 \\
\hline 19 & 1 & 0 & -7.757458 & 1.002861 & -0.431517 \\
\hline 20 & 1 & 0 & -5.786964 & 2.361430 & -1.111388 \\
\hline 21 & 1 & 0 & -0.667467 & -1.469180 & -1.250411 \\
\hline 22 & 6 & 0 & -1.814451 & 0.520376 & 1.258628 \\
\hline 23 & 1 & 0 & -2.738303 & 0.759804 & 1.774796 \\
\hline 24 & 6 & 0 & -4.361186 & 0.818676 & -0.668614 \\
\hline 25 & 1 & 0 & -3.501144 & 1.406092 & -0.972802 \\
\hline 26 & 6 & 0 & 4.051476 & 0.609909 & -0.563228 \\
\hline 27 & 6 & 0 & 4.086380 & -0.673674 & 0.253846 \\
\hline 28 & 6 & 0 & 5.096734 & 0.553629 & -1.692771 \\
\hline 29 & 1 & 0 & 5.035846 & 1.446109 & -2.279858 \\
\hline 30 & 1 & 0 & 6.075792 & 0.471606 & -1.268956 \\
\hline 31 & 1 & 0 & 4.904817 & -0.295933 & -2.314312 \\
\hline 32 & 6 & 0 & 5.234038 & -1.584289 & -0.220774 \\
\hline 33 & 1 & 0 & 6.163365 & -1.060942 & -0.134990 \\
\hline 34 & 1 & 0 & 5.263827 & -2.465718 & 0.385109 \\
\hline 35 & 1 & 0 & 5.072319 & -1.858906 & -1.242211 \\
\hline 36 & 6 & 0 & 4.343665 & -0.357447 & 1.738906 \\
\hline 37 & 1 & 0 & 3.929876 & -1.135271 & 2.346085 \\
\hline 38 & 1 & 0 & 5.397502 & -0.292093 & 1.912275 \\
\hline 39 & 1 & 0 & 3.882381 & 0.574742 & 1.990185 \\
\hline 40 & 6 & 0 & 4.402492 & 1.822865 & 0.318320 \\
\hline 41 & 1 & 0 & 5.400386 & 1.717025 & 0.689676 \\
\hline 42 & 1 & 0 & 4.331263 & 2.718563 & -0.262672 \\
\hline 43 & 1 & 0 & 3.719714 & 1.875774 & 1.140461 \\
\hline
\end{tabular}

\section{4,4,5,5-tetramethyl-2-(4-phenoxyphenyl)-1,3,2-dioxaborolane (diphenylether-4-Bpin)}

$\left(C_{1}\right.$ symmetry)

$\mathrm{E}(\mathrm{B} 3 \mathrm{LYP} / 6-31 \mathrm{G}(\mathrm{d}))=-949.20674692$ hartree

\begin{tabular}{lllcc} 
Center & Atomic & Atomic & \multicolumn{3}{c}{ Coordinates (Angstroms) } \\
Number & Number & Type & $X$ & $Y$ \\
\end{tabular}




\begin{tabular}{|c|c|c|c|c|c|}
\hline 1 & 5 & 0 & 2.073921 & -1.055435 & -0.424308 \\
\hline 2 & 8 & 0 & 2.817139 & -1.059938 & 0.904057 \\
\hline 3 & 8 & 0 & 2.877851 & -0.218484 & -1.408819 \\
\hline 4 & 8 & 0 & -3.131377 & 1.199008 & 0.225862 \\
\hline 5 & 6 & 0 & 0.659296 & -0.472347 & -0.249826 \\
\hline 6 & 6 & 0 & 0.419465 & 0.520604 & 0.702483 \\
\hline 7 & 6 & 0 & -0.861389 & 1.046848 & 0.867304 \\
\hline 8 & 6 & 0 & -1.906245 & 0.579450 & 0.067331 \\
\hline 9 & 6 & 0 & -0.393251 & -0.930647 & -1.044925 \\
\hline 10 & 1 & 0 & 1.230981 & 0.886406 & 1.325888 \\
\hline 11 & 1 & 0 & -0.215689 & -1.697058 & -1.794794 \\
\hline 12 & 6 & 0 & -4.307707 & 0.480049 & 0.129518 \\
\hline 13 & 6 & 0 & -5.393300 & 1.133564 & -0.457489 \\
\hline 14 & 6 & 0 & -6.631669 & 0.494937 & -0.514505 \\
\hline 15 & 6 & 0 & -6.788495 & -0.792267 & 0.004056 \\
\hline 16 & 6 & 0 & -5.695958 & -1.435000 & 0.589978 \\
\hline 17 & 1 & 0 & -5.250297 & 2.132947 & -0.856216 \\
\hline 18 & 1 & 0 & -7.475056 & 1.005833 & -0.971328 \\
\hline 19 & 1 & 0 & -7.753135 & -1.289000 & -0.045828 \\
\hline 20 & 1 & 0 & -5.809264 & -2.433421 & 1.004146 \\
\hline 21 & 1 & 0 & -1.068825 & 1.817697 & 1.602803 \\
\hline 22 & 6 & 0 & -1.678074 & -0.406990 & -0.896951 \\
\hline 23 & 1 & 0 & -2.494446 & -0.753844 & -1.521840 \\
\hline 24 & 6 & 0 & -4.453207 & -0.804658 & 0.661768 \\
\hline 25 & 1 & 0 & -3.606307 & -1.298194 & 1.127001 \\
\hline 26 & 6 & 0 & 4.165956 & 0.207593 & -0.719395 \\
\hline 27 & 6 & 0 & 4.096430 & -0.254065 & 0.729209 \\
\hline 28 & 6 & 0 & 5.326990 & -1.110007 & 1.082316 \\
\hline 29 & 1 & 0 & 6.215883 & -0.530416 & 0.945040 \\
\hline 30 & 1 & 0 & 5.261030 & -1.425654 & 2.102569 \\
\hline 31 & 1 & 0 & 5.359058 & -1.968664 & 0.444679 \\
\hline 32 & 6 & 0 & 4.100014 & 0.957945 & 1.679270 \\
\hline 33 & 1 & 0 & 3.661479 & 0.679224 & 2.614632 \\
\hline 34 & 1 & 0 & 5.106962 & 1.282180 & 1.839977 \\
\hline 35 & 1 & 0 & 3.534090 & 1.754543 & 1.243309 \\
\hline 36 & 6 & 0 & 4.339952 & 1.735958 & -0.793058 \\
\hline 37 & 1 & 0 & 5.242145 & 2.016827 & -0.291003 \\
\hline 38 & 1 & 0 & 4.392445 & 2.039982 & -1.817614 \\
\hline 39 & 1 & 0 & 3.506160 & 2.212979 & -0.321740 \\
\hline 40 & 6 & 0 & 5.382879 & -0.420992 & -1.423369 \\
\hline 41 & 1 & 0 & 5.393678 & -0.123033 & -2.450989 \\
\hline 42 & 1 & 0 & 6.281449 & -0.089375 & -0.946397 \\
\hline 43 & 1 & 0 & 5.319035 & -1.487312 & -1.361844 \\
\hline
\end{tabular}

9-phenyl-9H-carbazole (11) ( $C_{2}$ symmetry)

$\mathrm{E}(\mathrm{B} 3 \mathrm{LYP} / 6-31 \mathrm{G}(\mathrm{d}))=-748.52121302$ hartree

\begin{tabular}{|c|c|c|c|c|c|}
\hline \multirow{2}{*}{$\begin{array}{l}\text { Center } \\
\text { Number }\end{array}$} & \multirow{2}{*}{$\begin{array}{l}\text { Atomic } \\
\text { Number }\end{array}$} & \multirow{2}{*}{$\begin{array}{l}\text { Atomic } \\
\text { Type }\end{array}$} & \multicolumn{3}{|c|}{ Coordinates (Angstroms) } \\
\hline & & & $X$ & $\mathrm{Y}$ & Z \\
\hline 1 & 7 & 0 & 0.000000 & 0.000000 & 0.239858 \\
\hline 2 & 6 & 0 & -0.005427 & 3.037997 & -2.602200 \\
\hline 3 & 6 & 0 & 0.001977 & 3.427411 & -1.251664 \\
\hline 4 & 6 & 0 & 0.000000 & 2.486620 & -0.223993 \\
\hline 5 & 6 & 0 & -0.014536 & 1.134948 & -0.579330 \\
\hline 6 & 6 & 0 & -0.008332 & 0.723960 & -1.936876 \\
\hline 7 & 6 & 0 & -0.007172 & 1.690287 & -2.949720 \\
\hline & & & S68 & & \\
\hline
\end{tabular}




$\begin{array}{rrrrrr}8 & 1 & 0 & -0.005619 & 3.796730 & -3.379599 \\ 9 & 1 & 0 & 0.012118 & 4.484513 & -0.999879 \\ 10 & 1 & 0 & 0.015071 & 2.795368 & 0.816065 \\ 11 & 1 & 0 & -0.003039 & 1.390319 & -3.994492 \\ 12 & 6 & 0 & 0.014536 & -1.134948 & -0.579330 \\ 13 & 6 & 0 & 0.000000 & -2.486620 & -0.223993 \\ 14 & 6 & 0 & -0.001977 & -3.427411 & -1.251664 \\ 15 & 6 & 0 & 0.005427 & -3.037997 & -2.602200 \\ 16 & 6 & 0 & 0.007172 & -1.690287 & -2.949720 \\ 17 & 6 & 0 & 0.008332 & -0.723960 & -1.936876 \\ 18 & 1 & 0 & -0.015071 & -2.795368 & 0.816065 \\ 19 & 1 & 0 & -0.012118 & -4.484513 & -0.999879 \\ 20 & 1 & 0 & 0.005619 & -3.796730 & -3.379599 \\ 21 & 1 & 0 & 0.003039 & -1.390319 & -3.994492 \\ 22 & 6 & 0 & 0.000000 & 0.000000 & 1.661022 \\ 23 & 6 & 0 & -1.013667 & 0.663759 & 2.363884 \\ 24 & 6 & 0 & 1.013667 & -0.663759 & 2.363884 \\ 25 & 6 & 0 & -1.004450 & 0.669660 & 3.758519 \\ 26 & 1 & 0 & -1.806234 & 1.160788 & 1.813208 \\ 27 & 6 & 0 & 1.004450 & -0.669660 & 3.758519 \\ 28 & 1 & 0 & 1.806234 & -1.160788 & 1.813208 \\ 29 & 6 & 0.000000 & 0.000000 & 4.460094 \\ 30 & 1 & 0 & -1.793111 & 1.188163 & 4.296903 \\ 31 & 1 & 0 & 1.793111 & -1.188163 & 4.296903 \\ 32 & 1 & 0 & 0.000000 & 0.000000 & 5.546434 \\ ---------------------------------------------------------------------------------------------\end{array}$

1-(diiodoboranyl)-9-phenyl-9H-carbazole (9-phenylcarbazole-1-BI $\mathbf{H}_{2}\left(C_{1}\right.$ symmetry)

$\mathrm{E}(\mathrm{B} 3 \mathrm{LYP} / 631 \mathrm{LAN})=-795.59349106$ hartree

\begin{tabular}{rrrrrr} 
Center & Atomic & Atomic & \multicolumn{2}{c}{ Cordinates (Angstroms) } \\
Number & Number & Type & X & Y & Z \\
\hline 1 & 7 & 0 & -1.680124 & 0.402040 & -0.171235 \\
2 & 5 & 0 & 1.217696 & -0.792181 & 0.235154 \\
3 & 53 & 0 & 1.967294 & 0.385891 & 1.948148 \\
4 & 53 & 0 & 2.392252 & -0.894465 & -1.634086 \\
5 & 6 & 0 & -1.477913 & -3.641293 & 0.786531 \\
6 & 6 & 0 & -0.259065 & -2.950525 & 0.672974 \\
7 & 6 & 0 & -0.218889 & -1.595372 & 0.351808 \\
8 & 6 & 0 & -1.435383 & -0.940609 & 0.139864 \\
9 & 6 & 0 & -2.675045 & -1.618685 & 0.263689 \\
10 & 6 & 0 & -2.686556 & -2.980747 & 0.586643 \\
11 & 1 & 0 & -1.474487 & -4.698428 & 1.036489 \\
12 & 1 & 0 & 0.674189 & -3.481260 & 0.841255 \\
13 & 1 & 0 & -3.628866 & -3.513717 & 0.684316 \\
14 & 6 & 0 & -3.064865 & 0.591329 & -0.249020 \\
15 & 6 & 0 & -3.786864 & 1.746383 & -0.562167 \\
16 & 6 & 0 & -5.176818 & 1.654644 & -0.590641 \\
17 & 6 & 0 & -5.837406 & 0.443951 & -0.319550 \\
18 & 6 & 0 & -5.112380 & -0.705880 & -0.020684 \\
19 & 6 & 0 & -3.714394 & -0.641926 & 0.013800 \\
20 & 1 & 0 & -3.284179 & 2.682543 & -0.781677 \\
21 & 1 & 0 & -5.759684 & 2.539876 & -0.830633 \\
22 & 1 & 0 & -6.922678 & 0.406562 & -0.348097 \\
23 & 1 & 0 & -5.623997 & -1.643877 & 0.179205 \\
24 & 6 & 0 & -0.691263 & 1.402491 & -0.373617 \\
25 & 6 & 0 & 0.308145 & 1.218085 & -1.337614 \\
26 & 6 & 0 & -0.712552 & 2.576479 & 0.390197 \\
& & & 569 & & \\
& & 0 & & &
\end{tabular}




$\begin{array}{rrrrrr}27 & 6 & 0 & 1.282471 & 2.198015 & -1.526169 \\ 28 & 1 & 0 & 0.308262 & 0.314127 & -1.938700 \\ 29 & 6 & 0 & 0.253927 & 3.560099 & 0.181545 \\ 30 & 1 & 0 & -1.479002 & 2.705123 & 1.148122 \\ 31 & 6 & 0 & 1.256363 & 3.372942 & -0.772221 \\ 32 & 1 & 0 & 2.055642 & 2.047187 & -2.274597 \\ 33 & 1 & 0 & 0.229983 & 4.468935 & 0.776635 \\ 34 & 1 & 0 & 2.012250 & 4.137688 & -0.926922\end{array}$

2-(diiodoboranyl)-9-phenyl-9H-carbazole (9-phenylcarbazole-2-BI $)\left(C_{1}\right.$ symmetry) $\mathrm{E}(\mathrm{B} 3 \mathrm{LYP} / 631 \mathrm{LAN})=-795.60592477$ hartree

\begin{tabular}{|c|c|c|c|c|c|}
\hline \multirow{2}{*}{$\begin{array}{l}\text { Center } \\
\text { Number }\end{array}$} & \multirow{2}{*}{$\begin{array}{l}\text { Atomic } \\
\text { Number }\end{array}$} & \multirow{2}{*}{$\begin{array}{l}\text { Atomic } \\
\text { Type }\end{array}$} & \multicolumn{3}{|c|}{ Coordinates (Angstroms) } \\
\hline & & & $\mathrm{X}$ & $\mathrm{Y}$ & $\mathrm{Z}$ \\
\hline 1 & 7 & 0 & -2.848799 & 0.198638 & -0.042876 \\
\hline 2 & 5 & 0 & 2.277694 & -0.320678 & 0.031415 \\
\hline 3 & 53 & 0 & 3.264238 & 0.126727 & 1.957722 \\
\hline 4 & 53 & 0 & 3.367075 & -0.010575 & -1.866264 \\
\hline 5 & 6 & 0 & 0.497097 & -2.272703 & 0.021991 \\
\hline 6 & 6 & 0 & 0.727796 & -0.886239 & 0.010041 \\
\hline 7 & 6 & 0 & -0.325355 & 0.025565 & -0.016144 \\
\hline 8 & 6 & 0 & -1.626727 & -0.483907 & -0.035160 \\
\hline 9 & 6 & 0 & -1.878175 & -1.879617 & -0.009654 \\
\hline 10 & 6 & 0 & -0.801365 & -2.773755 & 0.015666 \\
\hline 11 & 1 & 0 & 1.340526 & -2.957036 & 0.040473 \\
\hline 12 & 1 & 0 & -0.138983 & 1.094461 & -0.015912 \\
\hline 13 & 1 & 0 & -0.978653 & -3.846018 & 0.034779 \\
\hline 14 & 6 & 0 & -3.881599 & -0.745997 & -0.023492 \\
\hline 15 & 6 & 0 & -5.265071 & -0.549652 & -0.054262 \\
\hline 16 & 6 & 0 & -6.080780 & -1.679147 & -0.046452 \\
\hline 17 & 6 & 0 & -5.538042 & -2.975298 & -0.013726 \\
\hline 18 & 6 & 0 & -4.159286 & -3.164538 & 0.004096 \\
\hline 19 & 6 & 0 & -3.316492 & -2.046828 & -0.004090 \\
\hline 20 & 1 & 0 & -5.691746 & 0.447354 & -0.088900 \\
\hline 21 & 1 & 0 & -7.159747 & -1.551507 & -0.068858 \\
\hline 22 & 1 & 0 & -6.201855 & -3.835139 & -0.006492 \\
\hline 23 & 1 & 0 & -3.740605 & -4.167539 & 0.019543 \\
\hline 24 & 6 & 0 & -3.012977 & 1.610091 & -0.066382 \\
\hline 25 & 6 & 0 & -2.425235 & 2.369166 & -1.086418 \\
\hline 26 & 6 & 0 & -3.763115 & 2.247134 & 0.930404 \\
\hline 27 & 6 & 0 & -2.580575 & 3.755098 & -1.100226 \\
\hline 28 & 1 & 0 & -1.860390 & 1.867422 & -1.865936 \\
\hline 29 & 6 & 0 & -3.930003 & 3.631413 & 0.898076 \\
\hline 30 & 1 & 0 & -4.200727 & 1.655052 & 1.728138 \\
\hline 31 & 6 & 0 & -3.336338 & 4.390037 & -0.112679 \\
\hline 32 & 1 & 0 & -2.120251 & 4.337516 & -1.893686 \\
\hline 33 & 1 & 0 & -4.514719 & 4.118406 & 1.673726 \\
\hline 34 & 1 & 0 & -3.461836 & 5.468954 & -0.130647 \\
\hline
\end{tabular}

3-(diiodoboranyl)-9-phenyl-9H-carbazole (9-phenylcarbazole-3-BI 2$)\left(C_{1}\right.$ symmetry) $\mathrm{E}(\mathrm{B} 3 \mathrm{LYP} / 631 \mathrm{LAN})=-795.60825992$ hartree

\begin{tabular}{|c|c|c|c|c|}
\hline Center & Atomic & Atomic & Coordinates (An & \\
\hline Number & Number & Type & $X \quad Y$ & Z \\
\hline
\end{tabular}




\begin{tabular}{|c|c|c|c|c|c|}
\hline 1 & 7 & 0 & 3.270128 & -0.010723 & -0.019047 \\
\hline 2 & 5 & 0 & -2.535971 & -0.185025 & 0.029377 \\
\hline 3 & 53 & 0 & -3.628087 & -0.037965 & 1.945037 \\
\hline 4 & 53 & 0 & -3.653800 & -0.326556 & -1.871814 \\
\hline 5 & 6 & 0 & -0.886011 & -0.189153 & 0.018574 \\
\hline 6 & 6 & 0 & -0.193873 & -1.408391 & 0.118825 \\
\hline 7 & 6 & 0 & 1.198546 & -1.456428 & 0.111045 \\
\hline 8 & 6 & 0 & 1.890622 & -0.247696 & -0.005104 \\
\hline 9 & 6 & 0 & 1.208670 & 0.992873 & -0.093483 \\
\hline 10 & 6 & 0 & -0.191037 & 1.012324 & -0.084183 \\
\hline 11 & 1 & 0 & -0.753785 & -2.335573 & 0.207109 \\
\hline 12 & 1 & 0 & 1.725433 & -2.400927 & 0.198136 \\
\hline 13 & 1 & 0 & -0.727303 & 1.955398 & -0.151829 \\
\hline 14 & 6 & 0 & 3.479424 & 1.369891 & -0.116451 \\
\hline 15 & 6 & 0 & 4.679099 & 2.082548 & -0.196398 \\
\hline 16 & 6 & 0 & 4.603489 & 3.469210 & -0.308878 \\
\hline 17 & 6 & 0 & 3.366385 & 4.135412 & -0.346222 \\
\hline 18 & 6 & 0 & 2.174977 & 3.419097 & -0.278871 \\
\hline 19 & 6 & 0 & 2.222120 & 2.024548 & -0.166381 \\
\hline 20 & 1 & 0 & 5.637936 & 1.575056 & -0.177526 \\
\hline 21 & 1 & 0 & 5.523075 & 4.044884 & -0.371511 \\
\hline 22 & 1 & 0 & 3.342133 & 5.217996 & -0.432537 \\
\hline 23 & 1 & 0 & 1.218786 & 3.934595 & -0.317668 \\
\hline 24 & 6 & 0 & 4.285195 & -1.002744 & 0.053348 \\
\hline 25 & 6 & 0 & 4.315682 & -2.046595 & -0.880224 \\
\hline 26 & 6 & 0 & 5.258748 & -0.940135 & 1.058528 \\
\hline 27 & 6 & 0 & 5.307734 & -3.023562 & -0.799629 \\
\hline 28 & 1 & 0 & 3.569042 & -2.079144 & -1.667570 \\
\hline 29 & 6 & 0 & 6.258933 & -1.910174 & 1.120020 \\
\hline 30 & 1 & 0 & 5.218746 & -0.138806 & 1.789771 \\
\hline 31 & 6 & 0 & 6.284434 & -2.956592 & 0.195935 \\
\hline 32 & 1 & 0 & 5.323954 & -3.831281 & -1.526276 \\
\hline 33 & 1 & 0 & 7.011795 & -1.854076 & 1.901519 \\
\hline 34 & 1 & 0 & 7.060353 & -3.714894 & 0.251274 \\
\hline
\end{tabular}

4-(diiodoboranyl)-9-phenyl-9H-carbazole (9-phenylcarbazole-4-BI $\left.{ }_{2}\right)\left(C_{1}\right.$ symmetry) $\mathrm{E}(\mathrm{B} 3 \mathrm{LYP} / 631 \mathrm{LAN})=-795.59498604$ hartree

\begin{tabular}{rrrrrr}
$\begin{array}{r}\text { Center } \\
\text { Number }\end{array}$ & $\begin{array}{c}\text { Atomic } \\
\text { Number }\end{array}$ & $\begin{array}{c}\text { Atomic } \\
\text { Type }\end{array}$ & \multicolumn{3}{c}{ Coordinates (Angstroms) } \\
\hline 1 & 7 & 0 & 2.844727 & 0.018514 & Z \\
2 & 5 & 0 & -2.059547 & -0.484791 & 0.147279 \\
3 & 53 & 0 & -3.059708 & 0.497769 & 1.855598 \\
4 & 53 & 0 & -3.072336 & -0.590947 & -1.814121 \\
5 & 6 & 0 & -0.408722 & -2.463371 & 0.738189 \\
6 & 6 & 0 & 0.870881 & -3.018466 & 0.911773 \\
7 & 6 & 0 & 2.024377 & -2.271540 & 0.682106 \\
8 & 6 & 0 & 1.869256 & -0.946149 & 0.266451 \\
9 & 6 & 0 & 0.585873 & -0.365707 & 0.099426 \\
10 & 6 & 0 & -0.556667 & -1.139121 & 0.336231 \\
11 & 1 & 0 & -1.288146 & -3.073446 & 0.923793 \\
12 & 1 & 0 & 0.966359 & -4.051725 & 1.234609 \\
13 & 1 & 0 & 3.008535 & -2.704699 & 0.827291 \\
14 & 6 & 0 & 2.199224 & 1.211816 & -0.356112 \\
15 & 6 & 0 & 2.747229 & 2.438030 & -0.742869 \\
16 & 6 & 0 & 1.867211 & 3.471784 & -1.056114 \\
17 & 6 & 0 & 0.474429 & 3.293628 & -0.992366
\end{tabular}




$\begin{array}{rrrrrr}18 & 6 & 0 & -0.065285 & 2.066373 & -0.618671 \\ 19 & 6 & 0 & 0.796350 & 1.010270 & -0.299493 \\ 20 & 1 & 0 & 3.821094 & 2.580906 & -0.803468 \\ 21 & 1 & 0 & 2.269955 & 4.434769 & -1.358473 \\ 22 & 1 & 0 & -0.184433 & 4.120679 & -1.241194 \\ 23 & 1 & 0 & -1.142567 & 1.926913 & -0.579162 \\ 24 & 6 & 0 & 4.250798 & -0.179812 & 0.046271 \\ 25 & 6 & 0 & 4.848221 & -1.199817 & -0.705253 \\ 26 & 6 & 0 & 5.044169 & 0.644021 & 0.854956 \\ 27 & 6 & 0 & 6.227200 & -1.397362 & -0.638028 \\ 28 & 1 & 0 & 4.229980 & -1.823012 & -1.343884 \\ 29 & 6 & 0 & 6.424836 & 0.452319 & 0.901154 \\ 30 & 1 & 0 & 4.572755 & 1.420913 & 1.448803 \\ 31 & 6 & 0 & 7.020143 & -0.570428 & 0.160092 \\ 32 & 1 & 0 & 6.683338 & -2.191743 & -1.222482 \\ 33 & 1 & 0 & 7.034032 & 1.096434 & 1.529394 \\ 34 & 1 & 0 & 8.094945 & -0.722029 & 0.204267\end{array}$

9-(2-(diiodoboranyl)phenyl)-9H-carbazole (9-phenylcarbazole-9a-BI 2$)\left(C_{1}\right.$ symmetry) $\mathrm{E}(\mathrm{B} 3 \mathrm{LYP} / 631 \mathrm{LAN})=-795.59381751$ hartree

\begin{tabular}{|c|c|c|c|c|c|}
\hline \multirow{2}{*}{$\begin{array}{l}\text { Center } \\
\text { Number }\end{array}$} & \multirow{2}{*}{$\begin{array}{l}\text { Atomic } \\
\text { Number }\end{array}$} & \multirow{2}{*}{$\begin{array}{l}\text { Atomic } \\
\text { Type }\end{array}$} & \multicolumn{3}{|c|}{ Coordinates (Angstroms) } \\
\hline & & & $\mathrm{X}$ & $\mathrm{Y}$ & Z \\
\hline 1 & 7 & 0 & 1.337127 & 0.343003 & -0.917118 \\
\hline 2 & 5 & 0 & -1.275736 & 0.593386 & 0.387435 \\
\hline 3 & 53 & 0 & -2.959452 & -0.643238 & -0.333647 \\
\hline 4 & 53 & 0 & -0.456630 & 0.270893 & 2.414542 \\
\hline 5 & 6 & 0 & 0.395657 & -3.663591 & -1.523404 \\
\hline 6 & 6 & 0 & -0.572139 & -2.716439 & -1.900127 \\
\hline 7 & 6 & 0 & -0.355204 & -1.350211 & -1.734110 \\
\hline 8 & 6 & 0 & 0.860184 & -0.947825 & -1.173388 \\
\hline 9 & 6 & 0 & 1.853517 & -1.889042 & -0.800182 \\
\hline 10 & 6 & 0 & 1.609166 & -3.256013 & -0.977171 \\
\hline 11 & 1 & 0 & 0.194507 & -4.721744 & -1.664396 \\
\hline 12 & 1 & 0 & -1.510194 & -3.053429 & -2.333085 \\
\hline 13 & 1 & 0 & -1.103652 & -0.624984 & -2.036012 \\
\hline 14 & 1 & 0 & 2.360760 & -3.988932 & -0.695245 \\
\hline 15 & 6 & 0 & 2.626478 & 0.238603 & -0.382270 \\
\hline 16 & 6 & 0 & 3.489470 & 1.246194 & 0.057614 \\
\hline 17 & 6 & 0 & 4.725571 & 0.862107 & 0.573107 \\
\hline 18 & 6 & 0 & 5.097133 & -0.490953 & 0.655656 \\
\hline 19 & 6 & 0 & 4.228268 & -1.490423 & 0.227603 \\
\hline 20 & 6 & 0 & 2.979455 & -1.132333 & -0.293821 \\
\hline 21 & 1 & 0 & 3.206468 & 2.292378 & 0.005827 \\
\hline 22 & 1 & 0 & 5.413925 & 1.627933 & 0.920442 \\
\hline 23 & 1 & 0 & 6.069248 & -0.756855 & 1.061015 \\
\hline 24 & 1 & 0 & 4.512799 & -2.536943 & 0.300969 \\
\hline 25 & 6 & 0 & 0.632426 & 1.553256 & -1.158763 \\
\hline 26 & 6 & 0 & -0.630212 & 1.757433 & -0.587652 \\
\hline 27 & 6 & 0 & 1.198020 & 2.546183 & -1.968893 \\
\hline 28 & 6 & 0 & -1.322675 & 2.942448 & -0.835365 \\
\hline 29 & 6 & 0 & 0.507390 & 3.736490 & -2.195448 \\
\hline 30 & 1 & 0 & 2.168839 & 2.374125 & -2.423153 \\
\hline 31 & 6 & 0 & -0.755527 & 3.936925 & -1.634698 \\
\hline 32 & 1 & 0 & -2.302454 & 3.093011 & -0.390336 \\
\hline 33 & 1 & 0 & 0.953241 & 4.502894 & -2.823563 \\
\hline & & & S72 & & \\
\hline
\end{tabular}


1. 9-(3-(diiodoboranyl)phenyl)-9H-carbazole (9-phenylcarbazole-9b-BI $\left.\mathbf{I}_{2}\right)\left(C_{1}\right.$ symmetry) $\mathrm{E}(\mathrm{B} 3 \mathrm{LYP} / 631 \mathrm{LAN})=-795.60159372$ hartree

\begin{tabular}{|c|c|c|c|c|c|}
\hline \multirow{2}{*}{$\begin{array}{l}\text { Center } \\
\text { Number }\end{array}$} & \multirow{2}{*}{$\begin{array}{l}\text { Atomic } \\
\text { Number }\end{array}$} & \multirow{2}{*}{$\begin{array}{c}\text { Atomic } \\
\text { Type }\end{array}$} & \multicolumn{3}{|c|}{ Coordinates (Angstroms) } \\
\hline & & & $X$ & $\mathrm{Y}$ & $\mathrm{Z}$ \\
\hline 1 & 7 & 0 & 2.650715 & 0.001170 & 0.436935 \\
\hline 2 & 5 & 0 & -2.413848 & -0.206210 & 0.151150 \\
\hline 3 & 53 & 0 & -3.585895 & 1.645194 & 0.438742 \\
\hline 4 & 53 & 0 & -3.129633 & -1.761253 & -1.246560 \\
\hline 5 & 6 & 0 & 4.164063 & 3.463877 & -1.302659 \\
\hline 6 & 6 & 0 & 2.865951 & 3.532942 & -0.768075 \\
\hline 7 & 6 & 0 & 2.267287 & 2.427162 & -0.168031 \\
\hline 8 & 6 & 0 & 2.999925 & 1.237953 & -0.117870 \\
\hline 9 & 6 & 0 & 4.316066 & 1.154070 & -0.639998 \\
\hline 10 & 6 & 0 & 4.893406 & 2.280228 & -1.238404 \\
\hline 11 & 1 & 0 & 4.601392 & 4.343871 & -1.765672 \\
\hline 12 & 1 & 0 & 2.315287 & 4.468386 & -0.819816 \\
\hline 13 & 1 & 0 & 1.268294 & 2.491248 & 0.250512 \\
\hline 14 & 1 & 0 & 5.900899 & 2.230754 & -1.643443 \\
\hline 15 & 6 & 0 & 3.729962 & -0.875370 & 0.274796 \\
\hline 16 & 6 & 0 & 3.842981 & -2.220841 & 0.635944 \\
\hline 17 & 6 & 0 & 5.036318 & -2.874697 & 0.336580 \\
\hline 18 & 6 & 0 & 6.093200 & -2.211727 & -0.310812 \\
\hline 19 & 6 & 0 & 5.969845 & -0.875255 & -0.679213 \\
\hline 20 & 6 & 0 & 4.781040 & -0.194500 & -0.391187 \\
\hline 21 & 1 & 0 & 3.028376 & -2.742297 & 1.127650 \\
\hline 22 & 1 & 0 & 5.147932 & -3.920865 & 0.608681 \\
\hline 23 & 1 & 0 & 7.011459 & -2.749856 & -0.528171 \\
\hline 24 & 1 & 0 & 6.784171 & -0.366338 & -1.188548 \\
\hline 25 & 6 & 0 & 1.411639 & -0.311312 & 1.058830 \\
\hline 26 & 6 & 0 & 0.209994 & -0.128918 & 0.362486 \\
\hline 27 & 6 & 0 & 1.387669 & -0.802792 & 2.370311 \\
\hline 28 & 6 & 0 & -1.004380 & -0.427474 & 0.979970 \\
\hline 29 & 1 & 0 & 0.236668 & 0.235092 & -0.659879 \\
\hline 30 & 6 & 0 & 0.170151 & -1.117533 & 2.973399 \\
\hline 31 & 1 & 0 & 2.321235 & -0.924640 & 2.910730 \\
\hline 32 & 6 & 0 & -1.028800 & -0.926765 & 2.283690 \\
\hline 33 & 1 & 0 & 0.159538 & -1.500002 & 3.990401 \\
\hline 34 & 1 & 0 & -1.975952 & -1.165626 & 2.759067 \\
\hline
\end{tabular}

9-(4-(diiodoboranyl)phenyl)-9H-carbazole (9-phenylcarbazole-9c-BI $\left.{ }_{2}\right)\left(C_{1}\right.$ symmetry) $\mathrm{E}(\mathrm{B} 3 \mathrm{LYP} / 631 \mathrm{LAN})=-795.60411474$ hartree

\begin{tabular}{cccccc} 
Center & Atomic & Atomic & \multicolumn{2}{c}{ Coordinates (Angstroms) } \\
Number & Number & Type & X & Y & Z \\
-1 & 7 & 0 & 0.000000 & 0.000000 & 3.078646 \\
2 & 5 & 0 & 0.000000 & 0.000000 & -2.791590 \\
3 & 53 & 0 & 0.000000 & 1.913916 & -3.896590 \\
4 & 53 & 0 & 0.000000 & -1.913916 & -3.896590 \\
5 & 6 & 0 & -1.858867 & -2.402929 & 5.920705 \\
6 & 6 & 0 & -2.090723 & -2.715884 & 4.570169 \\
7 & 6 & 0 & -1.517976 & -1.969525 & 3.542497 \\
& & & & &
\end{tabular}




$\begin{array}{rrrrrr}8 & 6 & 0 & -0.704351 & -0.890061 & 3.897834 \\ 9 & 6 & 0 & -0.448546 & -0.568325 & 5.255380 \\ 10 & 6 & 0 & -1.037529 & -1.334412 & 6.268225 \\ 11 & 1 & 0 & -2.322194 & -3.003766 & 6.698104 \\ 12 & 1 & 0 & -2.728007 & -3.559351 & 4.318384 \\ 13 & 1 & 0 & -1.694517 & -2.223269 & 2.502440 \\ 14 & 1 & 0 & -0.851138 & -1.099346 & 7.312997 \\ 15 & 6 & 0 & 0.704351 & 0.890061 & 3.897834 \\ 16 & 6 & 0 & 1.517976 & 1.969525 & 3.542497 \\ 17 & 6 & 0 & 2.090723 & 2.715884 & 4.570169 \\ 18 & 6 & 0 & 1.858867 & 2.402929 & 5.920705 \\ 19 & 6 & 0 & 1.037529 & 1.334412 & 6.268225 \\ 20 & 6 & 0 & 0.448546 & 0.568325 & 5.255380 \\ 21 & 1 & 0 & 1.694517 & 2.223269 & 2.502440 \\ 22 & 1 & 0 & 2.728007 & 3.559351 & 4.318384 \\ 23 & 1 & 0 & 2.322194 & 3.003766 & 6.698104 \\ 24 & 1 & 0 & 0.851138 & 1.099346 & 7.312997 \\ 25 & 6 & 0 & 0.000000 & 0.000000 & 1.657482 \\ 26 & 6 & 0 & -1.208071 & 0.093071 & 0.954621 \\ 27 & 6 & 0 & 1.208071 & -0.093071 & 0.954621 \\ 28 & 6 & 0 & -1.204373 & 0.082771 & -0.440014 \\ 29 & 1 & 0 & -2.139238 & 0.183229 & 1.505297 \\ 30 & 6 & 0 & 1.204373 & -0.082771 & -0.440014 \\ 31 & 1 & 0 & 2.139238 & -0.183229 & 1.505297 \\ 32 & 6 & 0 & 0.000000 & 0.000000 & -1.141590 \\ 33 & 1 & 0 & -2.145555 & 0.153535 & -0.978399 \\ 34 & 1 & 0 & 2.145555 & -0.153535 & -0.978399 \\ -------------------------------- \\ \end{array}$

9-(phenyl)-2-(4,4,5,5-tetramethyl-1,3,2-dioxaborolane-2-yl)-9H-carbazole (9-phenylcarbazole2-Bpin) ( $C_{1}$ symmetry) $\mathrm{E}(\mathrm{B} 3 \mathrm{LYP} / 6-31 \mathrm{G}(\mathrm{d}))=-1159.21442679$ hartree

\begin{tabular}{rrrrrr} 
Center & Atomic & Atomic & \multicolumn{2}{c}{ Coordinates (Angstroms) } \\
Number & Number & Type & X & Y & Z \\
\hline 1 & 7 & 0 & -2.552160 & 0.115874 & -0.023581 \\
2 & 5 & 0 & 2.447093 & 0.860243 & 0.009773 \\
3 & 8 & 0 & 3.273108 & 0.571112 & 1.254825 \\
4 & 8 & 0 & 3.317733 & 0.578926 & -1.206694 \\
5 & 6 & 0 & 1.314554 & -1.418631 & -0.035627 \\
6 & 6 & 0 & 1.183260 & -0.019354 & -0.015443 \\
7 & 6 & 0 & -0.068128 & 0.593223 & -0.013840 \\
8 & 6 & 0 & -1.196274 & -0.231495 & -0.038485 \\
9 & 6 & 0 & -1.082477 & -1.645333 & -0.045408 \\
10 & 6 & 0 & 0.187211 & -2.234764 & -0.047029 \\
11 & 1 & 0 & 2.304960 & -1.864858 & -0.038154 \\
12 & 1 & 0 & 0.290026 & -3.316867 & -0.052692 \\
13 & 6 & 0 & -3.309095 & -1.061592 & -0.021850 \\
14 & 6 & 0 & -4.697073 & -1.225005 & -0.040675 \\
15 & 6 & 0 & -5.196931 & -2.525428 & -0.055887 \\
16 & 6 & 0 & -4.340654 & -3.640065 & -0.057802 \\
17 & 6 & 0 & -2.959216 & -3.470763 & -0.052012 \\
18 & 6 & 0 & -2.430196 & -2.174816 & -0.037019 \\
19 & 1 & 0 & -5.364749 & -0.369808 & -0.049062 \\
20 & 1 & 0 & -6.272869 & -2.677496 & -0.069525 \\
& & & & & \\
& 6 & & & &
\end{tabular}




$\begin{array}{rrrrrr}21 & 1 & 0 & -4.762560 & -4.641024 & -0.068036 \\ 22 & 1 & 0 & -2.297985 & -4.333429 & -0.063367 \\ 23 & 1 & 0 & -0.160972 & 1.673937 & 0.011856 \\ 24 & 6 & 0 & -3.071848 & 1.438670 & -0.011726 \\ 25 & 6 & 0 & -2.709640 & 2.345165 & -1.016331 \\ 26 & 6 & 0 & -3.947899 & 1.840756 & 1.004659 \\ 27 & 6 & 0 & -3.213739 & 3.645401 & -0.995295 \\ 28 & 1 & 0 & -2.045054 & 2.021924 & -1.811482 \\ 29 & 6 & 0 & -4.463344 & 3.136693 & 1.006919 \\ 30 & 1 & 0 & -4.210088 & 1.139359 & 1.790614 \\ 31 & 6 & 0 & -4.094994 & 4.044086 & 0.011672 \\ 32 & 1 & 0 & -2.926824 & 4.343491 & -1.776983 \\ 33 & 1 & 0 & -5.143801 & 3.440802 & 1.797605 \\ 34 & 1 & 0 & -4.492086 & 5.055209 & 0.020765 \\ 35 & 6 & 0 & 4.682836 & 0.210763 & 0.808869 \\ 36 & 6 & 0 & 4.691358 & 0.148979 & -0.711830 \\ 37 & 6 & 0 & 5.111731 & -1.141386 & 1.408320 \\ 38 & 1 & 0 & 6.175773 & -1.160857 & 1.519382 \\ 39 & 1 & 0 & 4.650823 & -1.269484 & 2.365427 \\ 40 & 1 & 0 & 4.806595 & -1.933296 & 0.756653 \\ 41 & 6 & 0 & 5.009310 & -1.280329 & -1.188887 \\ 42 & 1 & 0 & 4.857166 & -1.345632 & -2.246000 \\ 43 & 1 & 0 & 6.028004 & -1.514394 & -0.960027 \\ 44 & 1 & 0 & 4.363674 & -1.974053 & -0.692096 \\ 45 & 6 & 0 & 5.784671 & 1.070881 & -1.283114 \\ 46 & 1 & 0 & 6.744937 & 0.730508 & -0.956104 \\ 47 & 1 & 0 & 5.744541 & 1.051784 & -2.352190 \\ 48 & 1 & 0 & 5.624174 & 2.070891 & -0.937977 \\ 49 & 6 & 0 & 5.687825 & 1.265952 & 1.307038 \\ 50 & 1 & 0 & 5.646813 & 1.321442 & 2.374811 \\ 51 & 1 & 0 & 6.675528 & 0.989831 & 1.001914 \\ 52 & 1 & 0 & 5.439407 & 2.219735 & 0.890520 \\ ------------------------------------------------------------------------------------------------\end{array}$

9-(phenyl)-3-(4,4,5,5-tetramethyl-1,3,2-dioxaborolane-2-yl)-9H-carbazole (9-phenylcarbazole3-Bpin) ( $C_{1}$ symmetry)

$\mathrm{E}(\mathrm{B} 3 \mathrm{LYP} / 6-31 \mathrm{G}(\mathrm{d}))=-1159.21526578$ hartree

\begin{tabular}{rrrrrr}
$\begin{array}{c}\text { Center } \\
\text { Number }\end{array}$ & $\begin{array}{c}\text { Atomic } \\
\text { Number }\end{array}$ & $\begin{array}{c}\text { Atomic } \\
\text { Type }\end{array}$ & \multicolumn{3}{c}{ Coordinates (Angstroms) } \\
-1 & 7 & 0 & 2.644579 & -0.083180 & -0.021562 \\
2 & 5 & 0 & -2.919102 & 1.150953 & -0.030832 \\
3 & 8 & 0 & -3.603075 & 0.642075 & 1.230188 \\
4 & 8 & 0 & -3.626075 & 0.534564 & -1.229291 \\
5 & 6 & 0 & -1.427557 & 0.767694 & -0.028515 \\
6 & 6 & 0 & -1.058931 & -0.588332 & 0.001952 \\
7 & 6 & 0 & 0.278756 & -0.977974 & 0.002807 \\
8 & 6 & 0 & 1.249231 & 0.027193 & -0.033200 \\
9 & 6 & 0 & 0.895394 & 1.400668 & -0.050595 \\
10 & 6 & 0 & -0.456365 & 1.764377 & -0.051208 \\
11 & 1 & 0 & -1.831474 & -1.352156 & 0.028484 \\
12 & 1 & 0 & -0.742667 & 2.812897 & -0.064817 \\
13 & 6 & 0 & 3.189087 & 1.206307 & -0.032298 \\
14 & 6 & 0 & 4.528636 & 1.604383 & -0.058495 \\
15 & 6 & 0 & 4.798782 & 2.970932 & -0.085997 \\
16 & 6 & 0 & 3.764561 & 3.922756 & -0.092798 \\
& & 0 & &
\end{tabular}




\begin{tabular}{|c|c|c|c|c|c|}
\hline 17 & 6 & 0 & 2.432424 & 3.519887 & -0.079619 \\
\hline 18 & 6 & 0 & 2.132779 & 2.152762 & -0.052242 \\
\hline 19 & 1 & 0 & 5.332651 & 0.875849 & -0.063179 \\
\hline 20 & 1 & 0 & 5.832840 & 3.304540 & -0.105546 \\
\hline 21 & 1 & 0 & 4.009111 & 4.980976 & -0.112656 \\
\hline 22 & 1 & 0 & 1.633423 & 4.256715 & -0.094762 \\
\hline 23 & 1 & 0 & 0.555058 & -2.026663 & 0.036481 \\
\hline 24 & 6 & 0 & 3.382782 & -1.297540 & -0.001726 \\
\hline 25 & 6 & 0 & 3.177852 & -2.261009 & -0.997663 \\
\hline 26 & 6 & 0 & 4.317724 & -1.535425 & 1.013887 \\
\hline 27 & 6 & 0 & 3.896865 & -3.455729 & -0.968752 \\
\hline 28 & 1 & 0 & 2.465404 & -2.062805 & -1.792368 \\
\hline 29 & 6 & 0 & 5.047126 & -2.724127 & 1.023939 \\
\hline 30 & 1 & 0 & 4.458513 & -0.792960 & 1.793214 \\
\hline 31 & 6 & 0 & 4.836326 & -3.689452 & 0.037393 \\
\hline 32 & 1 & 0 & 3.731164 & -4.199126 & -1.743729 \\
\hline 33 & 1 & 0 & 5.771927 & -2.900806 & 1.813962 \\
\hline 34 & 1 & 0 & 5.400449 & -4.617714 & 0.052588 \\
\hline 35 & 6 & 0 & -4.808567 & -0.271916 & -0.712220 \\
\hline 36 & 6 & 0 & -4.753267 & -0.261257 & 0.808715 \\
\hline 37 & 6 & 0 & -4.539124 & -1.687854 & 1.347754 \\
\hline 38 & 1 & 0 & -3.951869 & -1.647106 & 2.241271 \\
\hline 39 & 1 & 0 & -5.487536 & -2.133417 & 1.564293 \\
\hline 40 & 1 & 0 & -4.029180 & -2.274245 & 0.612226 \\
\hline 41 & 6 & 0 & -6.083486 & 0.248926 & 1.393377 \\
\hline 42 & 1 & 0 & -6.882789 & -0.381998 & 1.064819 \\
\hline 43 & 1 & 0 & -6.032781 & 0.233047 & 2.462057 \\
\hline 44 & 1 & 0 & -6.259132 & 1.250206 & 1.059481 \\
\hline 45 & 6 & 0 & -6.131915 & 0.343515 & -1.203742 \\
\hline 46 & 1 & 0 & -6.133718 & 0.378005 & -2.273185 \\
\hline 47 & 1 & 0 & -6.951339 & -0.255297 & -0.864820 \\
\hline 48 & 1 & 0 & -6.230158 & 1.335443 & -0.814734 \\
\hline 49 & 6 & 0 & -4.754810 & -1.715418 & -1.246085 \\
\hline 50 & 1 & 0 & -5.603627 & -2.259855 & -0.888318 \\
\hline 51 & 1 & 0 & -4.765598 & -1.700658 & -2.315929 \\
\hline 52 & 1 & 0 & -3.857853 & -2.188694 & -0.904941 \\
\hline
\end{tabular}

(4,4,5,5-tetramethyl-1,3,2-dioxaborolane-2-yl)ferrocene (ferrocene-Bpin) ( $C_{1}$ symmetry) $\mathrm{E}(\mathrm{B} 3 \mathrm{LYP} / 6-31 \mathrm{G}(\mathrm{d}))=-2061.39918813$ hartree

\begin{tabular}{|c|c|c|c|c|c|}
\hline \multirow{2}{*}{$\begin{array}{l}\text { Center } \\
\text { Number }\end{array}$} & \multirow{2}{*}{$\begin{array}{l}\text { Atomic } \\
\text { Number }\end{array}$} & \multirow{2}{*}{$\begin{array}{l}\text { Atomic } \\
\text { Type }\end{array}$} & \multicolumn{3}{|c|}{ Coordinates (Angstroms) } \\
\hline & & & $\mathrm{X}$ & Y & $\mathrm{Z}$ \\
\hline 1 & 26 & 0 & -2.240022 & 0.121788 & -0.007156 \\
\hline 2 & 5 & 0 & 0.899410 & -0.515251 & 0.029394 \\
\hline 3 & 8 & 0 & 1.779629 & -0.284720 & 1.249646 \\
\hline 4 & 8 & 0 & 1.779375 & -0.419739 & -1.208585 \\
\hline 5 & 6 & 0 & -0.849727 & 1.031277 & -1.212945 \\
\hline 6 & 1 & 0 & -0.610366 & 0.740892 & -2.227781 \\
\hline 7 & 6 & 0 & -1.855926 & 1.966043 & -0.822761 \\
\hline 8 & 1 & 0 & -2.513210 & 2.508646 & -1.489896 \\
\hline 9 & 6 & 0 & -1.856701 & 2.044017 & 0.602857 \\
\hline 10 & 1 & 0 & -2.514676 & 2.656106 & 1.206120 \\
\hline 11 & 6 & 0 & -0.850981 & 1.157443 & 1.093753 \\
\hline 12 & 1 & 0 & -0.612737 & 0.979487 & 2.134465 \\
\hline 13 & 6 & 0 & -2.624117 & -1.722467 & 0.808449 \\
\hline 14 & 1 & 0 & -1.966833 & -2.265070 & 1.475584 \\
\hline & & & S76 & & \\
\hline
\end{tabular}




$\begin{array}{rrrrrr}15 & 6 & 0 & -2.623342 & -1.800442 & -0.617169 \\ 16 & 1 & 0 & -1.965368 & -2.412530 & -1.220432 \\ 17 & 6 & 0 & -3.629062 & -0.913867 & -1.108065 \\ 18 & 1 & 0 & -3.867307 & -0.735911 & -2.148777 \\ 19 & 6 & 0 & -4.251406 & -0.287960 & 0.014162 \\ 20 & 1 & 0 & -5.044235 & 0.447757 & -0.026509 \\ 21 & 6 & 0 & -3.630316 & -0.787702 & 1.198633 \\ 22 & 1 & 0 & -3.869678 & -0.497316 & 2.213469 \\ 23 & 6 & 0 & -0.228637 & 0.531535 & -0.028474 \\ 24 & 6 & 0 & 3.217761 & -0.232723 & -0.748006 \\ 25 & 6 & 0 & 3.208416 & -0.080819 & 0.766343 \\ 26 & 6 & 0 & 3.715567 & 1.316571 & 1.168462 \\ 27 & 1 & 0 & 3.394105 & 1.539727 & 2.164335 \\ 28 & 1 & 0 & 4.784606 & 1.333733 & 1.126480 \\ 29 & 1 & 0 & 3.320362 & 2.047164 & 0.493965 \\ 30 & 6 & 0 & 4.151339 & -1.109599 & 1.417577 \\ 31 & 1 & 0 & 5.149984 & -0.949189 & 1.068466 \\ 32 & 1 & 0 & 4.122819 & -0.996357 & 2.481186 \\ 33 & 1 & 0 & 3.836363 & -2.098051 & 1.155561 \\ 34 & 6 & 0 & 4.073140 & -1.445673 & -1.158710 \\ 35 & 1 & 0 & 4.020095 & -1.576795 & -2.219320 \\ 36 & 1 & 0 & 5.089990 & -1.279062 & -0.870340 \\ 37 & 1 & 0 & 3.703658 & -2.323927 & -0.671830 \\ 38 & 6 & 0 & 3.842634 & 1.007821 & -1.412974 \\ 39 & 1 & 0 & 4.853859 & 1.118977 & -1.081357 \\ 40 & 1 & 0 & 3.827015 & 0.888537 & -2.476189 \\ 41 & 1 & 0 & 3.281195 & 1.877887 & -1.143398\end{array}$

(5,6,7,8-tetrahydronaphthalen-2-yl)boronic acid (3) ( $C_{1}$ symmetry)

$\mathrm{E}(\mathrm{B} 3 \mathrm{LYP} / 6-31 \mathrm{G}(\mathrm{d}))=-564.32101721$ hartree

\begin{tabular}{|c|c|c|c|c|c|}
\hline \multirow{2}{*}{$\begin{array}{l}\text { Center } \\
\text { Number }\end{array}$} & \multirow{2}{*}{$\begin{array}{l}\text { Atomic } \\
\text { Number }\end{array}$} & \multirow{2}{*}{$\begin{array}{r}\text { Atomic } \\
\text { Type }\end{array}$} & \multicolumn{3}{|c|}{ Coordinates (Angstroms) } \\
\hline & & & $\mathrm{X}$ & $\mathrm{Y}$ & $\mathrm{Z}$ \\
\hline 1 & 5 & 0 & 3.044061 & -0.226457 & -0.030377 \\
\hline 2 & 8 & 0 & 3.522212 & -1.689995 & -0.062416 \\
\hline 3 & 8 & 0 & 4.159945 & 0.834775 & -0.016547 \\
\hline 4 & 6 & 0 & 1.134885 & 1.504433 & 0.037565 \\
\hline 5 & 6 & 0 & -0.210293 & 1.834115 & 0.056901 \\
\hline 6 & 6 & 0 & -1.207882 & 0.846942 & 0.024755 \\
\hline 7 & 6 & 0 & -0.823853 & -0.506849 & -0.033059 \\
\hline 8 & 6 & 0 & 0.535739 & -0.823809 & -0.046905 \\
\hline 9 & 6 & 0 & 1.555197 & 0.153100 & -0.013401 \\
\hline 10 & 1 & 0 & 1.875660 & 2.296146 & 0.062444 \\
\hline 11 & 1 & 0 & -0.505066 & 2.880577 & 0.098368 \\
\hline 12 & 1 & 0 & 0.813586 & -1.873521 & -0.085785 \\
\hline 13 & 6 & 0 & -3.256270 & -1.178499 & 0.338326 \\
\hline 14 & 1 & 0 & -3.275946 & -1.049583 & 1.429726 \\
\hline 15 & 1 & 0 & -3.985794 & -1.961104 & 0.099398 \\
\hline 16 & 6 & 0 & -3.636153 & 0.144485 & -0.335824 \\
\hline 17 & 1 & 0 & -4.662787 & 0.433377 & -0.082160 \\
\hline 18 & 1 & 0 & -3.603269 & 0.018442 & -1.427088 \\
\hline 19 & 6 & 0 & -2.667079 & 1.256196 & 0.086785 \\
\hline 20 & 1 & 0 & -2.894444 & 1.555803 & 1.121695 \\
\hline & & & S77 & & \\
\hline
\end{tabular}




$\begin{array}{rrrrrr}21 & 1 & 0 & -2.819784 & 2.156659 & -0.521746 \\ 22 & 6 & 0 & -1.856666 & -1.620131 & -0.107803 \\ 23 & 1 & 0 & -1.911706 & -1.979527 & -1.146547 \\ 24 & 1 & 0 & -1.515985 & -2.476451 & 0.487127 \\ 25 & 1 & 0 & 3.388497 & -2.089759 & 0.800086 \\ 26 & 1 & 0 & 5.013724 & 0.403089 & -0.095974\end{array}$

potassium trifluoro(5,6,7,8-tetrahydro-2-naphthalenyl)borate $(4)\left(C_{1}\right.$ symmetry)

$\mathrm{E}(\mathrm{B} 3 \mathrm{LYP} / 6-31 \mathrm{G}(\mathrm{d}))=-1312.23080974$ hartree

\begin{tabular}{|c|c|c|c|c|c|}
\hline \multirow{2}{*}{$\begin{array}{l}\text { Center } \\
\text { Number }\end{array}$} & \multirow{2}{*}{$\begin{array}{l}\text { Atomic } \\
\text { Number }\end{array}$} & \multirow{2}{*}{$\begin{array}{c}\text { Atomic } \\
\text { Type }\end{array}$} & \multicolumn{3}{|c|}{ Coordinates (Angstroms) } \\
\hline & & & $\mathrm{X}$ & Y & $\mathrm{Z}$ \\
\hline 1 & 5 & 0 & 2.189497 & -0.105046 & 0.124938 \\
\hline 2 & 9 & 0 & 2.513373 & -0.841298 & -0.959284 \\
\hline 3 & 9 & 0 & 2.916837 & 1.032253 & 0.130222 \\
\hline 4 & 9 & 0 & 2.452418 & -0.812553 & 1.244227 \\
\hline 5 & 19 & 0 & 3.347182 & -0.202006 & -0.246229 \\
\hline 6 & 6 & 0 & 0.690409 & 0.244545 & 0.078909 \\
\hline 7 & 6 & 0 & -0.262247 & -0.766145 & 0.065708 \\
\hline 8 & 6 & 0 & -1.639199 & -0.482104 & 0.023911 \\
\hline 9 & 6 & 0 & -2.055085 & 0.851852 & -0.004962 \\
\hline 10 & 6 & 0 & -1.083861 & 1.869182 & 0.008667 \\
\hline 11 & 6 & 0 & 0.274045 & 1.580033 & 0.050003 \\
\hline 12 & 1 & 0 & 0.057037 & -1.806910 & 0.088091 \\
\hline 13 & 1 & 0 & -1.412612 & 2.906990 & -0.013940 \\
\hline 14 & 1 & 0 & 1.004184 & 2.385217 & 0.059723 \\
\hline 15 & 6 & 0 & -4.587752 & 0.145468 & -0.065841 \\
\hline 16 & 1 & 0 & -5.211229 & 0.286985 & -0.954680 \\
\hline 17 & 1 & 0 & -5.259313 & 0.309609 & 0.783186 \\
\hline 18 & 6 & 0 & -4.123842 & -1.342526 & -0.033634 \\
\hline 19 & 1 & 0 & -4.545580 & -1.848083 & -0.908467 \\
\hline 20 & 1 & 0 & -4.593665 & -1.825459 & 0.829399 \\
\hline 21 & 6 & 0 & -2.605280 & -1.649946 & 0.012385 \\
\hline 22 & 1 & 0 & -2.345190 & -2.288400 & -0.842323 \\
\hline 23 & 1 & 0 & -2.393301 & -2.265763 & 0.896482 \\
\hline 24 & 6 & 0 & -3.513330 & 1.262625 & -0.050657 \\
\hline 25 & 1 & 0 & -3.699583 & 1.924137 & 0.805793 \\
\hline 26 & 1 & 0 & -3.651472 & 1.901500 & -0.933012 \\
\hline
\end{tabular}

10-mesityl-2,8-dimethyl-5-(p-tolyl)-10H-dibenzo $[\boldsymbol{b}, \boldsymbol{e}][1,4]$ oxaborine $(5)\left(C_{\mathrm{s}}\right.$ symmetry) $\mathrm{E}(\mathrm{B} 3 \mathrm{LYP} / 6-31 \mathrm{G}(\mathrm{d}))=-990.44817480$ hartree

\begin{tabular}{|c|c|c|c|c|c|}
\hline \multirow{2}{*}{$\begin{array}{l}\text { Center } \\
\text { Number }\end{array}$} & \multirow{2}{*}{$\begin{array}{l}\text { Atomic } \\
\text { Number }\end{array}$} & \multirow{2}{*}{$\begin{array}{l}\text { Atomic } \\
\text { Type }\end{array}$} & \multicolumn{3}{|c|}{ Coordinates (Angstroms) } \\
\hline & & & $X$ & $\mathrm{Y}^{-}$ & $\mathrm{Z}$ \\
\hline 1 & 5 & 0 & 0.0000820 & 0.1996680 & 0.0000000 \\
\hline 2 & 8 & 0 & 0.0006760 & 3.1059440 & 0.0000000 \\
\hline 3 & 6 & 0 & -3.7471340 & 1.2754470 & 0.0000000 \\
\hline 4 & 6 & 0 & -1.1948380 & 2.4423040 & 0.0000000 \\
\hline 5 & 6 & 0 & -2.3304770 & 3.2646940 & 0.0000000 \\
\hline 6 & 6 & 0 & -3.5850790 & 2.6784550 & 0.0000000 \\
\hline & & & S78 & & \\
\hline
\end{tabular}




\begin{tabular}{|c|c|c|c|c|c|}
\hline 7 & 6 & 0 & 1.1958710 & 2.4416580 & 0.0000000 \\
\hline 8 & 6 & 0 & 1.2935780 & 1.0373020 & 0.0000000 \\
\hline 9 & 6 & 0 & 2.6006960 & 0.4911800 & 0.0000000 \\
\hline 10 & 1 & 0 & 2.6944800 & -0.5926780 & 0.0000000 \\
\hline 11 & 6 & 0 & 3.7478410 & 1.2740690 & 0.0000000 \\
\hline 12 & 6 & 0 & 3.5861430 & 2.6771220 & 0.0000000 \\
\hline 13 & 6 & 0 & 2.3317380 & 3.2637150 & 0.0000000 \\
\hline 14 & 1 & 0 & -2.2057260 & 4.3430320 & 0.0000000 \\
\hline 15 & 1 & 0 & -4.4667110 & 3.3163160 & 0.0000000 \\
\hline 16 & 1 & 0 & 4.4679290 & 3.3147710 & 0.0000000 \\
\hline 17 & 1 & 0 & 2.2072940 & 4.3420860 & 0.0000000 \\
\hline 18 & 6 & 0 & 5.1296660 & 0.6629120 & 0.0000000 \\
\hline 19 & 1 & 0 & 5.7054880 & 0.9693210 & 0.8826840 \\
\hline 20 & 1 & 0 & 5.0790480 & -0.4302260 & 0.0000000 \\
\hline 21 & 1 & 0 & 5.7054880 & 0.9693210 & -0.8826840 \\
\hline 22 & 6 & 0 & -5.1290660 & 0.6645520 & 0.0000000 \\
\hline 23 & 1 & 0 & -5.0786400 & -0.4286070 & 0.0000000 \\
\hline 24 & 1 & 0 & -5.7049750 & 0.9712170 & 0.8823920 \\
\hline 25 & 1 & 0 & -5.7049750 & 0.9712170 & -0.8823920 \\
\hline 26 & 6 & 0 & -1.2929650 & 1.0379600 & 0.0000000 \\
\hline 27 & 6 & 0 & -2.6002170 & 0.4922560 & 0.0000000 \\
\hline 28 & 1 & 0 & -2.6943390 & -0.5915870 & 0.0000000 \\
\hline 29 & 6 & 0 & -0.0007560 & -1.3795980 & 0.0000000 \\
\hline 30 & 6 & 0 & 0.0014940 & -2.1019970 & 1.2147940 \\
\hline 31 & 6 & 0 & 0.0014940 & -2.1019970 & -1.2147940 \\
\hline 32 & 6 & 0 & 0.0051390 & -3.5006680 & 1.1973510 \\
\hline 33 & 6 & 0 & 0.0051390 & -3.5006680 & -1.1973510 \\
\hline 34 & 6 & 0 & 0.0036210 & -4.2216570 & 0.0000000 \\
\hline 35 & 1 & 0 & 0.0107400 & -4.0408520 & 2.1430510 \\
\hline 36 & 1 & 0 & 0.0107400 & -4.0408520 & -2.1430510 \\
\hline 37 & 6 & 0 & -0.0256740 & -5.7328100 & 0.0000000 \\
\hline 38 & 1 & 0 & 0.4753900 & -6.1440180 & 0.8835520 \\
\hline 39 & 1 & 0 & -1.0562810 & -6.1134100 & 0.0000000 \\
\hline 40 & 1 & 0 & 0.4753900 & -6.1440180 & -0.8835520 \\
\hline 41 & 6 & 0 & 0.0051390 & -1.3713570 & -2.5420960 \\
\hline 42 & 1 & 0 & 0.0068410 & -2.0723050 & -3.3832000 \\
\hline 43 & 1 & 0 & -0.8756770 & -0.7254140 & -2.6487340 \\
\hline 44 & 1 & 0 & 0.8862830 & -0.7252910 & -2.6450430 \\
\hline 45 & 6 & 0 & 0.0051390 & -1.3713570 & 2.5420960 \\
\hline 46 & 1 & 0 & 0.8862830 & -0.7252910 & 2.6450430 \\
\hline 47 & 1 & 0 & -0.8756770 & -0.7254140 & 2.6487340 \\
\hline 48 & 1 & 0 & 0.0068410 & -2.0723050 & 3.3832000 \\
\hline
\end{tabular}

10-mesityl-2,8-dimethyl-5-(p-tolyl)-5,10-dihydrodibenzo $[\boldsymbol{b}, \boldsymbol{e}][1,4]$ azaborine $(6)\left(C_{\mathrm{s}}\right.$ symmetry $)$ $\mathrm{E}(\mathrm{B} 3 \mathrm{LYP} / 6-31 \mathrm{G}(\mathrm{d}))=-1240.95147562$ hartree

\begin{tabular}{|c|c|c|c|c|c|}
\hline \multirow{2}{*}{$\begin{array}{l}\text { Center } \\
\text { Number }\end{array}$} & \multirow{2}{*}{$\begin{array}{l}\text { Atomic } \\
\text { Number }\end{array}$} & \multirow{2}{*}{$\begin{array}{l}\text { Atomic } \\
\text { Type }\end{array}$} & \multicolumn{3}{|c|}{ Coordinates (Angstroms) } \\
\hline & & & $\mathrm{X}$ & $\mathrm{Y}$ & Z \\
\hline 1 & 7 & 0 & 0.000606 & 1.731668 & 0.000000 \\
\hline 2 & 5 & 0 & 0.000525 & -1.177457 & 0.000000 \\
\hline 3 & 6 & 0 & -1.293941 & -0.354309 & 0.000000 \\
\hline 4 & 6 & 0 & -2.574058 & -0.960867 & 0.000000 \\
\hline 5 & 6 & 0 & -3.760747 & -0.244600 & 0.000000 \\
\hline 6 & 6 & 0 & -3.658537 & 1.161875 & 0.000000 \\
\hline 7 & 6 & 0 & -2.436694 & 1.810089 & 0.000000 \\
\hline 8 & 6 & 0 & -1.233023 & 1.065527 & 0.000000 \\
\hline & & & S79 & & \\
\hline
\end{tabular}




\begin{tabular}{|c|c|c|c|c|c|}
\hline 9 & 1 & 0 & -2.609866 & -2.048246 & 0.000000 \\
\hline 10 & 1 & 0 & -4.566542 & 1.762438 & 0.000000 \\
\hline 11 & 1 & 0 & -2.413523 & 2.892679 & 0.000000 \\
\hline 12 & 6 & 0 & 1.234232 & 1.065444 & 0.000000 \\
\hline 13 & 6 & 0 & 1.295043 & -0.354388 & 0.000000 \\
\hline 14 & 6 & 0 & 2.437932 & 1.809965 & 0.000000 \\
\hline 15 & 6 & 0 & 2.575126 & -0.961046 & 0.000000 \\
\hline 16 & 6 & 0 & 3.659726 & 1.161647 & 0.000000 \\
\hline 17 & 1 & 0 & 2.414939 & 2.892562 & 0.000000 \\
\hline 18 & 6 & 0 & 3.761853 & -0.244837 & 0.000000 \\
\hline 19 & 1 & 0 & 2.610915 & -2.048431 & 0.000000 \\
\hline 20 & 1 & 0 & 4.567759 & 1.762161 & 0.000000 \\
\hline 21 & 6 & 0 & 0.000389 & 3.174088 & 0.000000 \\
\hline 22 & 6 & 0 & 0.002878 & 3.875930 & 1.207341 \\
\hline 23 & 6 & 0 & 0.002878 & 3.875930 & -1.207341 \\
\hline 24 & 6 & 0 & 0.006621 & 5.270440 & 1.201914 \\
\hline 25 & 1 & 0 & 0.006199 & 3.325445 & 2.143531 \\
\hline 26 & 6 & 0 & 0.006621 & 5.270440 & -1.201914 \\
\hline 27 & 1 & 0 & 0.006199 & 3.325445 & -2.143531 \\
\hline 28 & 6 & 0 & 0.005258 & 5.991281 & 0.000000 \\
\hline 29 & 1 & 0 & 0.012471 & 5.807156 & 2.147719 \\
\hline 30 & 1 & 0 & 0.012471 & 5.807156 & -2.147719 \\
\hline 31 & 6 & 0 & -5.109907 & -0.923374 & 0.000000 \\
\hline 32 & 1 & 0 & -5.702122 & -0.646239 & 0.881916 \\
\hline 33 & 1 & 0 & -5.702122 & -0.646239 & -0.881916 \\
\hline 34 & 1 & 0 & -5.004248 & -2.012782 & 0.000000 \\
\hline 35 & 6 & 0 & 5.110964 & -0.923702 & 0.000000 \\
\hline 36 & 1 & 0 & 5.703192 & -0.646560 & 0.881924 \\
\hline 37 & 1 & 0 & 5.005245 & -2.013101 & 0.000000 \\
\hline 38 & 1 & 0 & 5.703192 & -0.646560 & -0.881924 \\
\hline 39 & 6 & 0 & -0.022271 & 7.501958 & 0.000000 \\
\hline 40 & 1 & 0 & 0.473338 & 7.912101 & -0.886228 \\
\hline 41 & 1 & 0 & -1.053594 & 7.879208 & 0.000000 \\
\hline 42 & 1 & 0 & 0.473338 & 7.912101 & 0.886228 \\
\hline 43 & 6 & 0 & 0.000163 & -2.761296 & 0.000000 \\
\hline 44 & 6 & 0 & 0.002559 & -3.485990 & 1.213771 \\
\hline 45 & 6 & 0 & 0.002559 & -3.485990 & -1.213771 \\
\hline 46 & 6 & 0 & 0.006034 & -4.884969 & 1.196874 \\
\hline 47 & 6 & 0 & 0.006034 & -4.884969 & -1.196874 \\
\hline 48 & 6 & 0 & 0.004261 & -5.606212 & 0.000000 \\
\hline 49 & 1 & 0 & 0.011678 & -5.425195 & 2.142716 \\
\hline 50 & 1 & 0 & 0.011678 & -5.425195 & -2.142716 \\
\hline 51 & 6 & 0 & -0.025501 & -7.117500 & 0.000000 \\
\hline 52 & 1 & 0 & 0.470590 & -7.528941 & 0.885971 \\
\hline 53 & 1 & 0 & -1.056011 & -7.498724 & 0.000000 \\
\hline 54 & 1 & 0 & 0.470590 & -7.528941 & -0.885971 \\
\hline 55 & 6 & 0 & 0.005814 & -2.756807 & 2.541838 \\
\hline 56 & 1 & 0 & 0.886388 & -2.110020 & 2.643842 \\
\hline 57 & 1 & 0 & -0.874894 & -2.110818 & 2.647887 \\
\hline 58 & 1 & 0 & 0.007951 & -3.458845 & 3.382302 \\
\hline 59 & 6 & 0 & 0.005814 & -2.756807 & -2.541838 \\
\hline 60 & 1 & 0 & -0.874894 & -2.110818 & -2.647887 \\
\hline 61 & 1 & 0 & 0.886388 & -2.110020 & -2.643842 \\
\hline 62 & 1 & 0 & 0.007951 & -3.458845 & -3.382302 \\
\hline
\end{tabular}




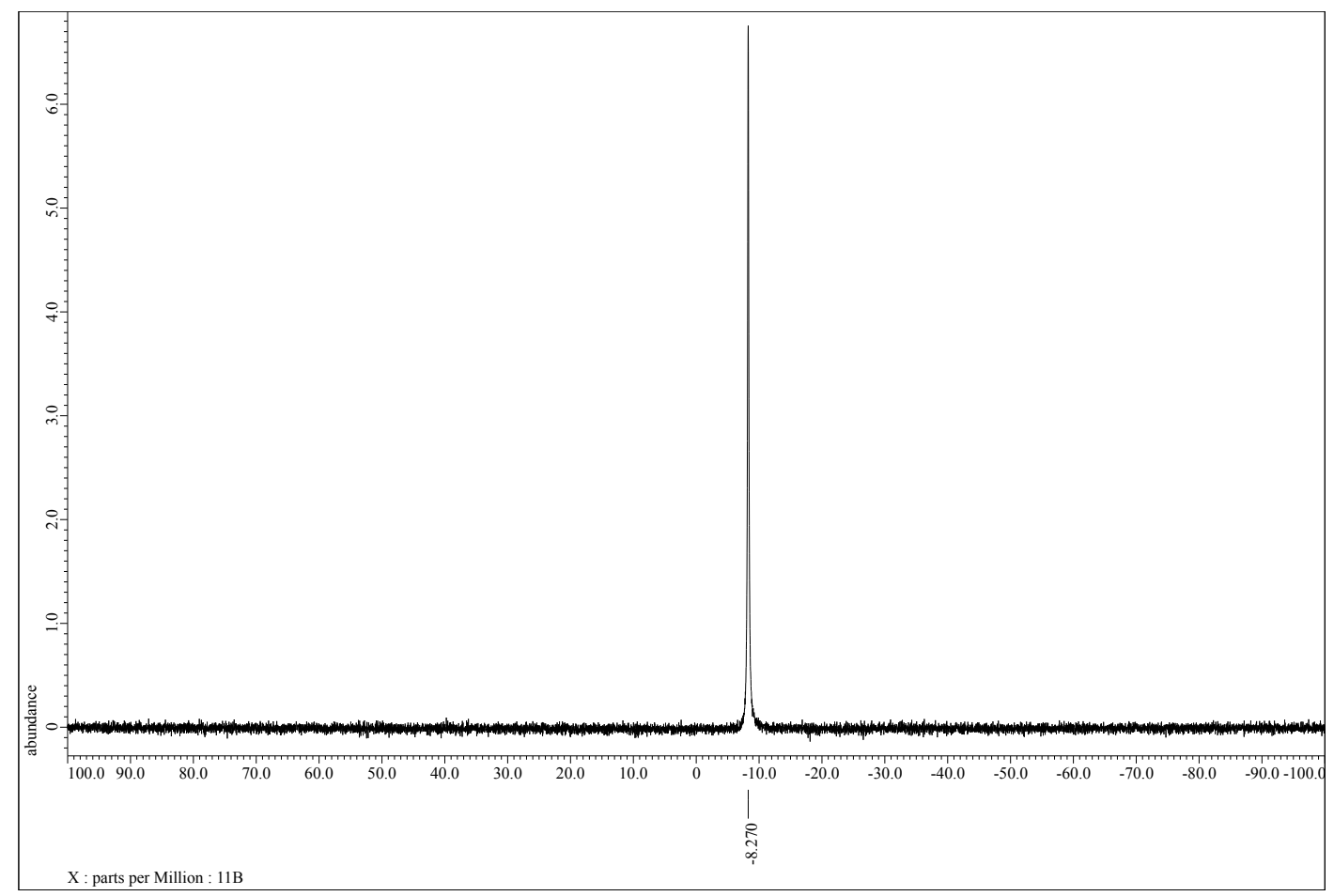

Figure S4. ${ }^{11} \mathrm{~B}$ NMR spectrum of $\mathrm{BI}_{3}$ in $\mathrm{CDCl}_{3}$ at $25^{\circ} \mathrm{C}$.

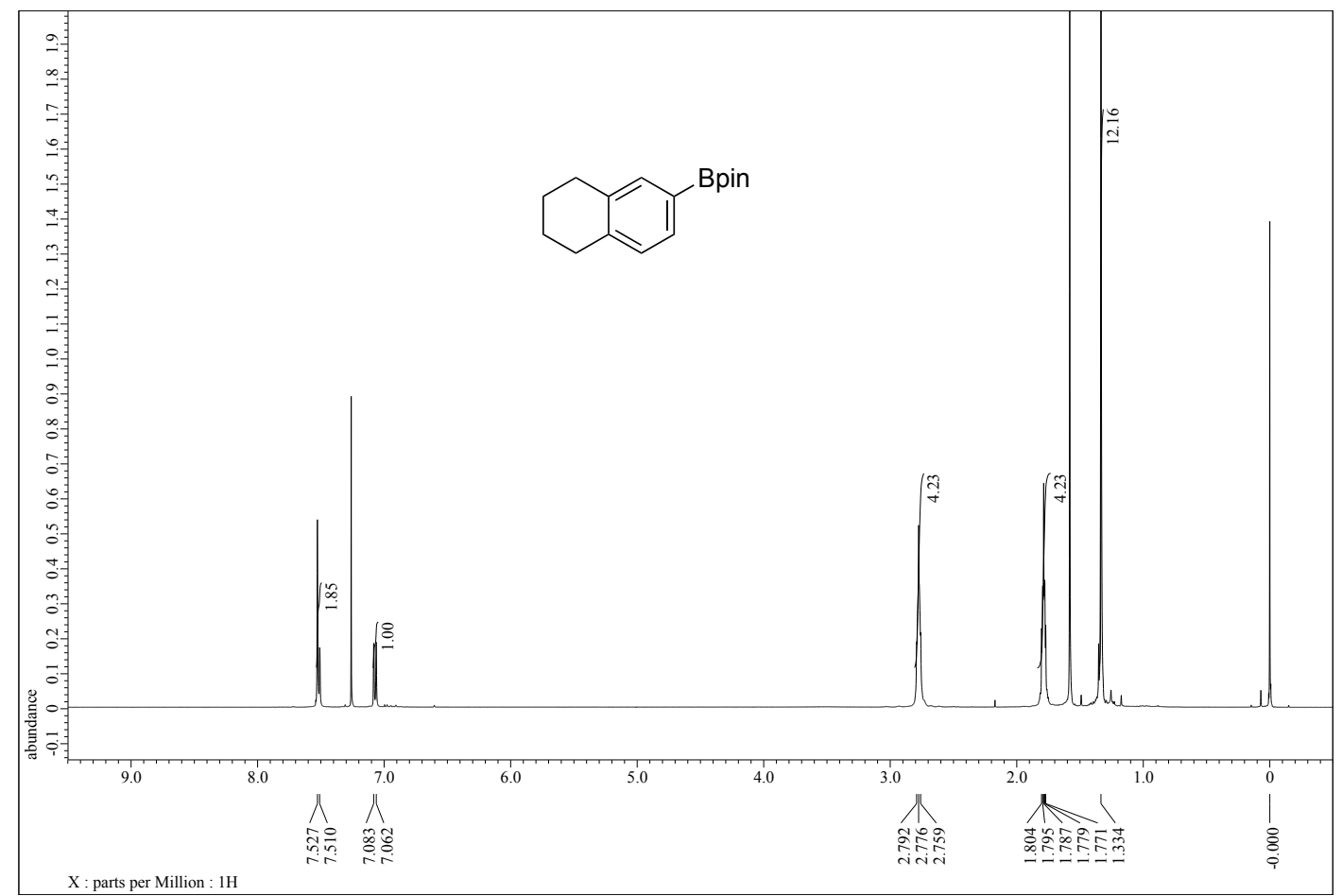

Figure S5. ${ }^{1} \mathrm{H}$ NMR spectrum of $\mathbf{2 a}$ in $\mathrm{CDCl}_{3}$ at $25^{\circ} \mathrm{C}$. 


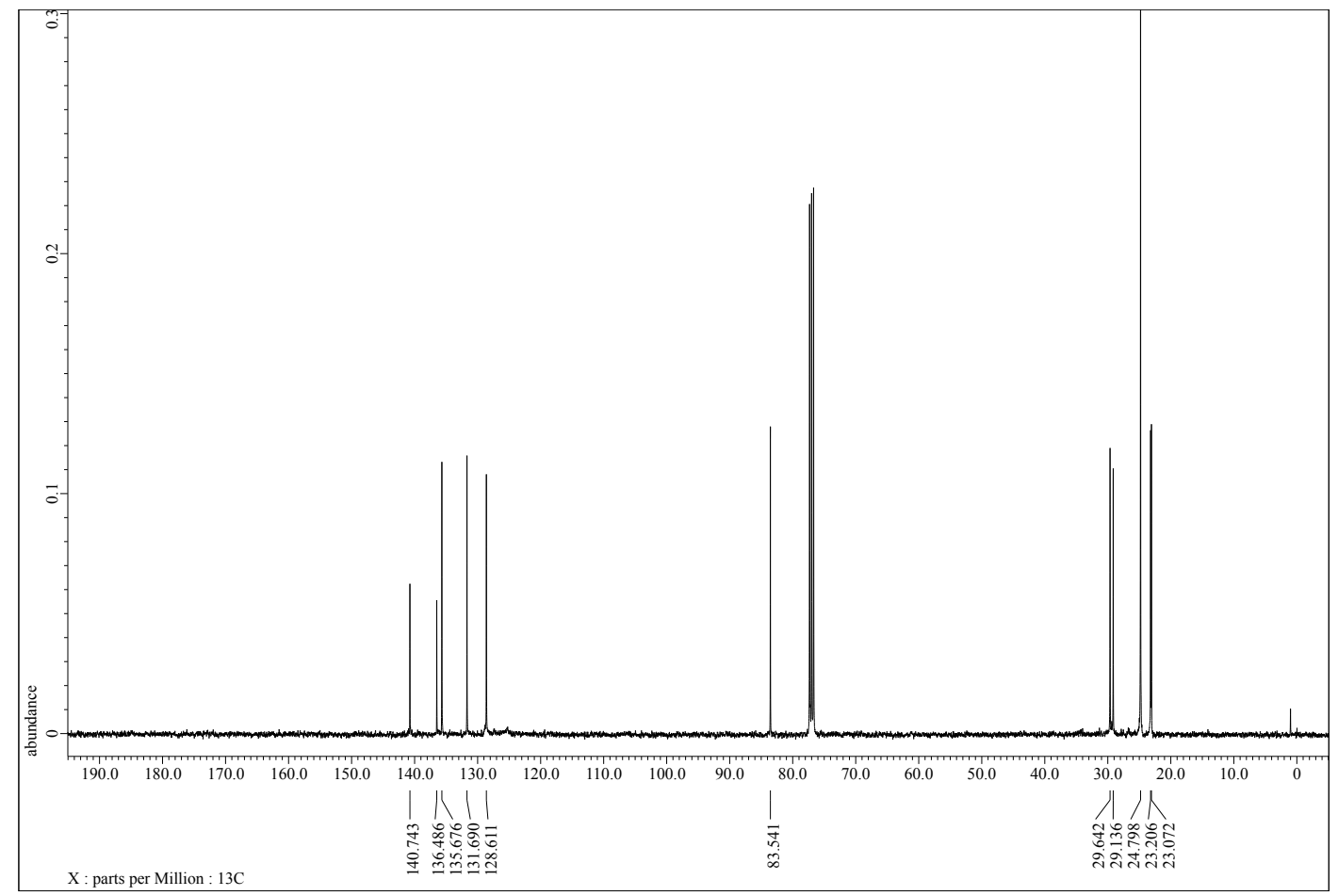

Figure S6. ${ }^{13} \mathrm{C}$ NMR spectrum of $\mathbf{2 a}$ in $\mathrm{CDCl}_{3}$ at $25{ }^{\circ} \mathrm{C}$.

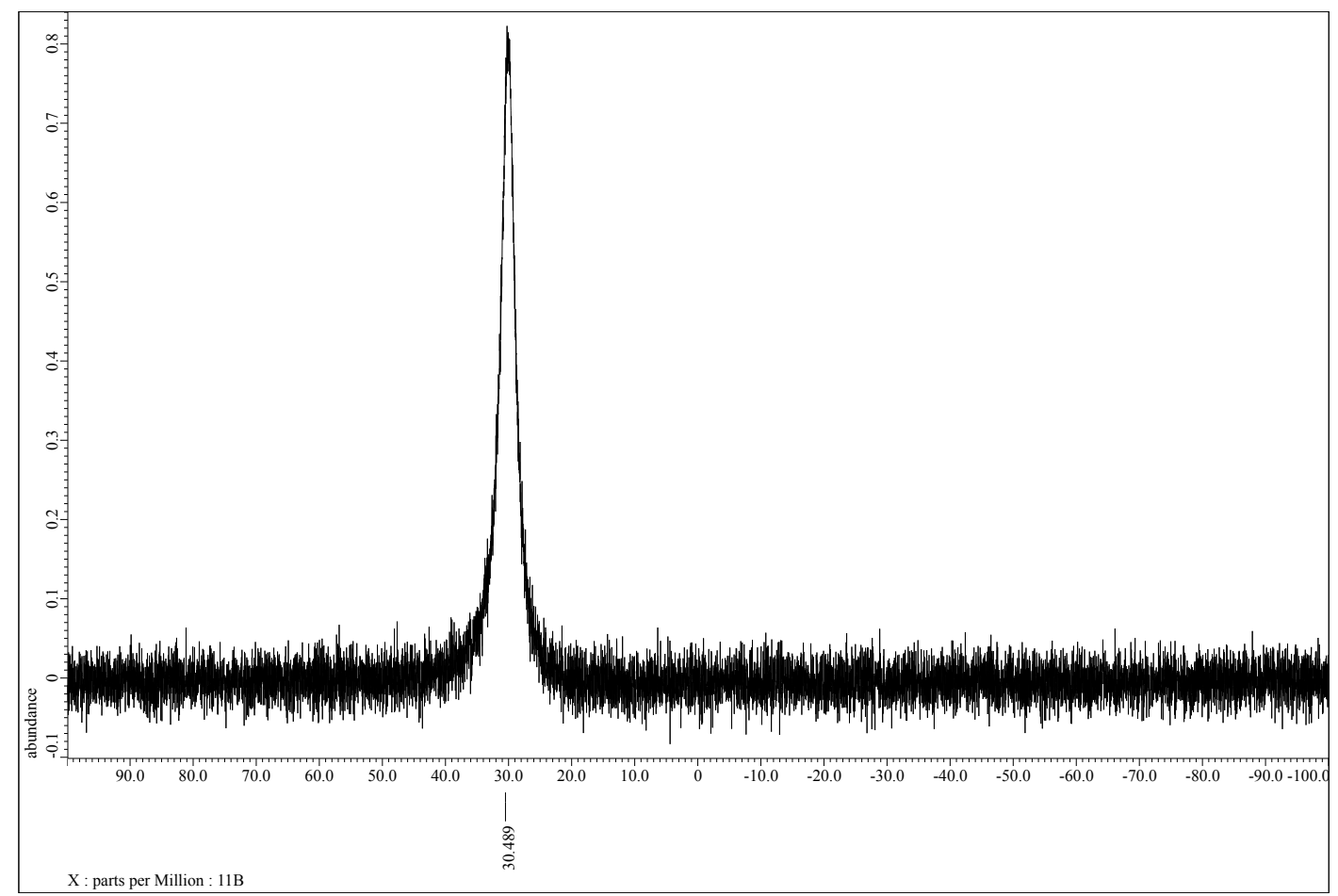

Figure S7. ${ }^{11} \mathrm{~B}$ NMR spectrum of $\mathbf{2 a}$ in $\mathrm{CDCl}_{3}$ at $25^{\circ} \mathrm{C}$. 


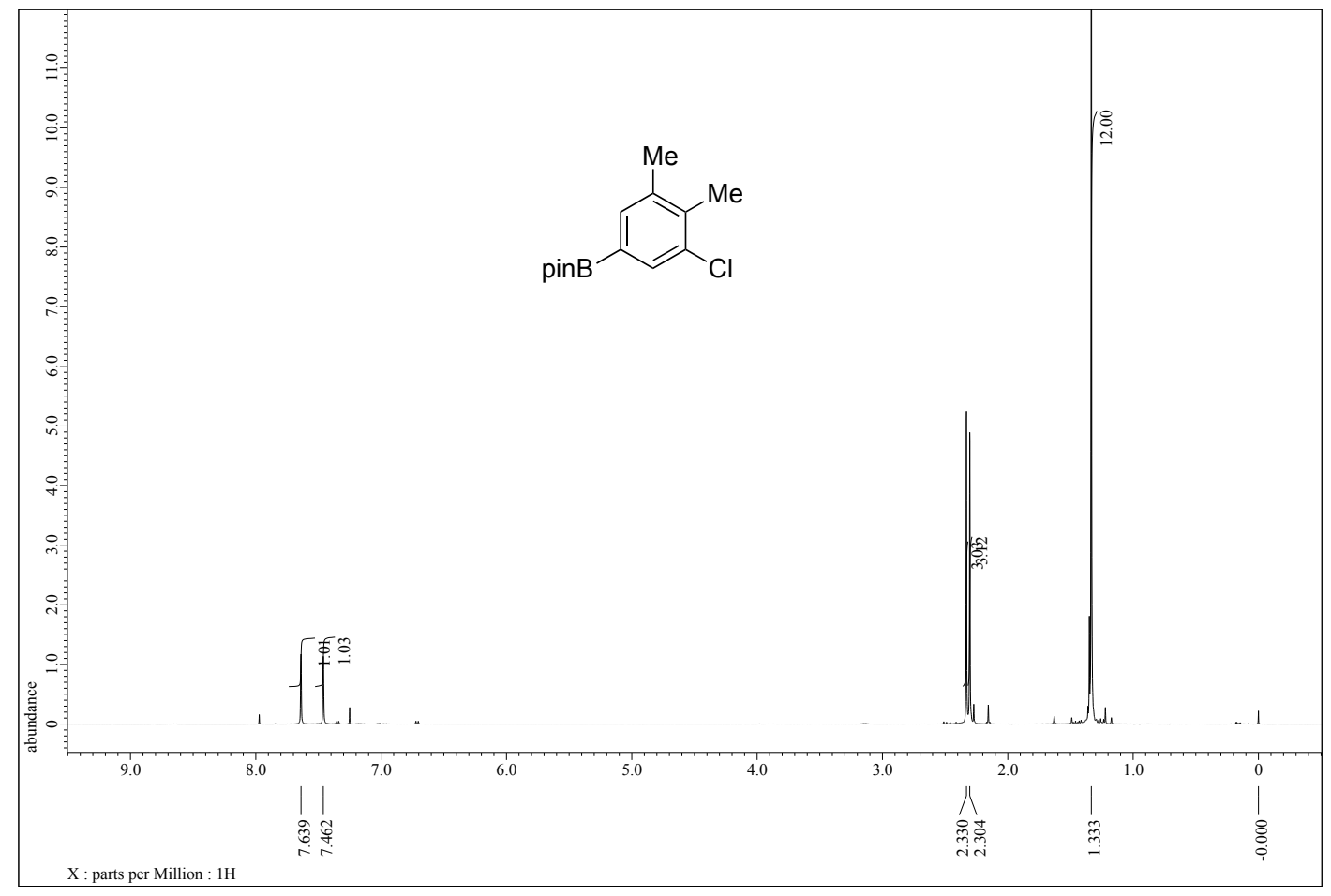

Figure S8. ${ }^{1} \mathrm{H}$ NMR spectrum of $\mathbf{2 b}$ in $\mathrm{CDCl}_{3}$ at $25{ }^{\circ} \mathrm{C}$.

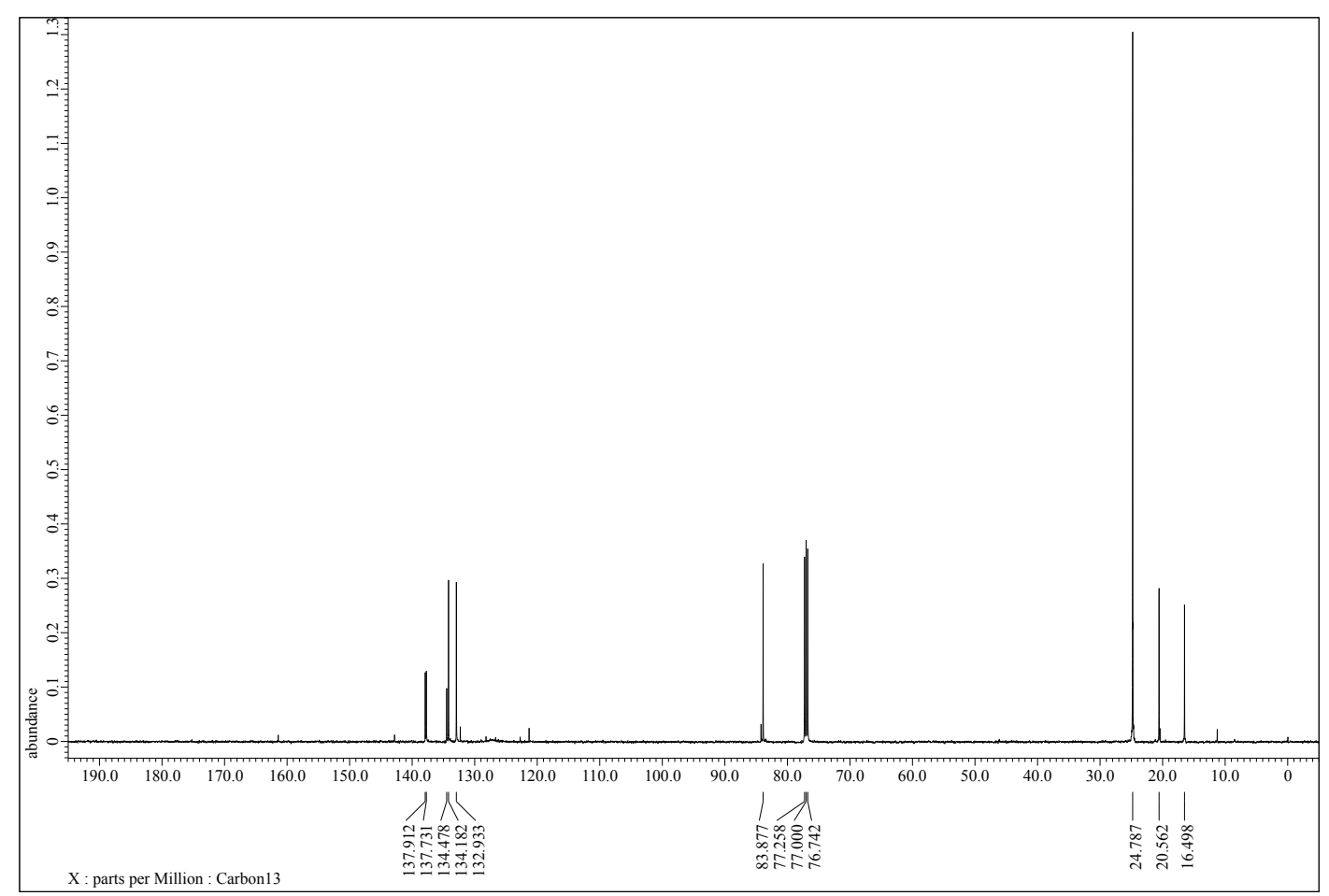

Figure S9. ${ }^{13} \mathrm{C}$ NMR spectrum of $\mathbf{2 b}$ in $\mathrm{CDCl}_{3}$ at $25{ }^{\circ} \mathrm{C}$. 


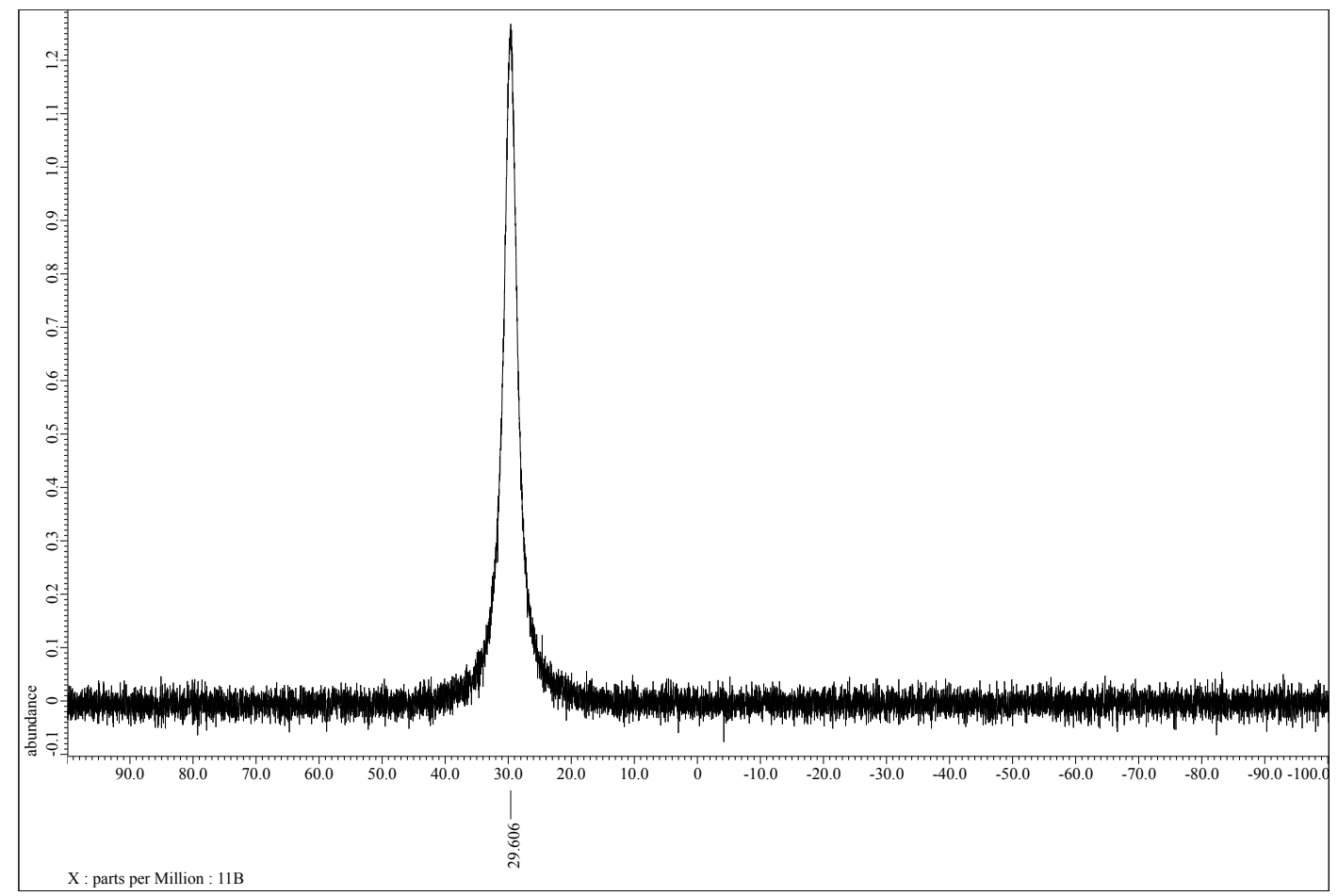

Figure S10. ${ }^{11} \mathrm{~B}$ NMR spectrum of $\mathbf{2 b}$ in $\mathrm{CDCl}_{3}$ at $25^{\circ} \mathrm{C}$.

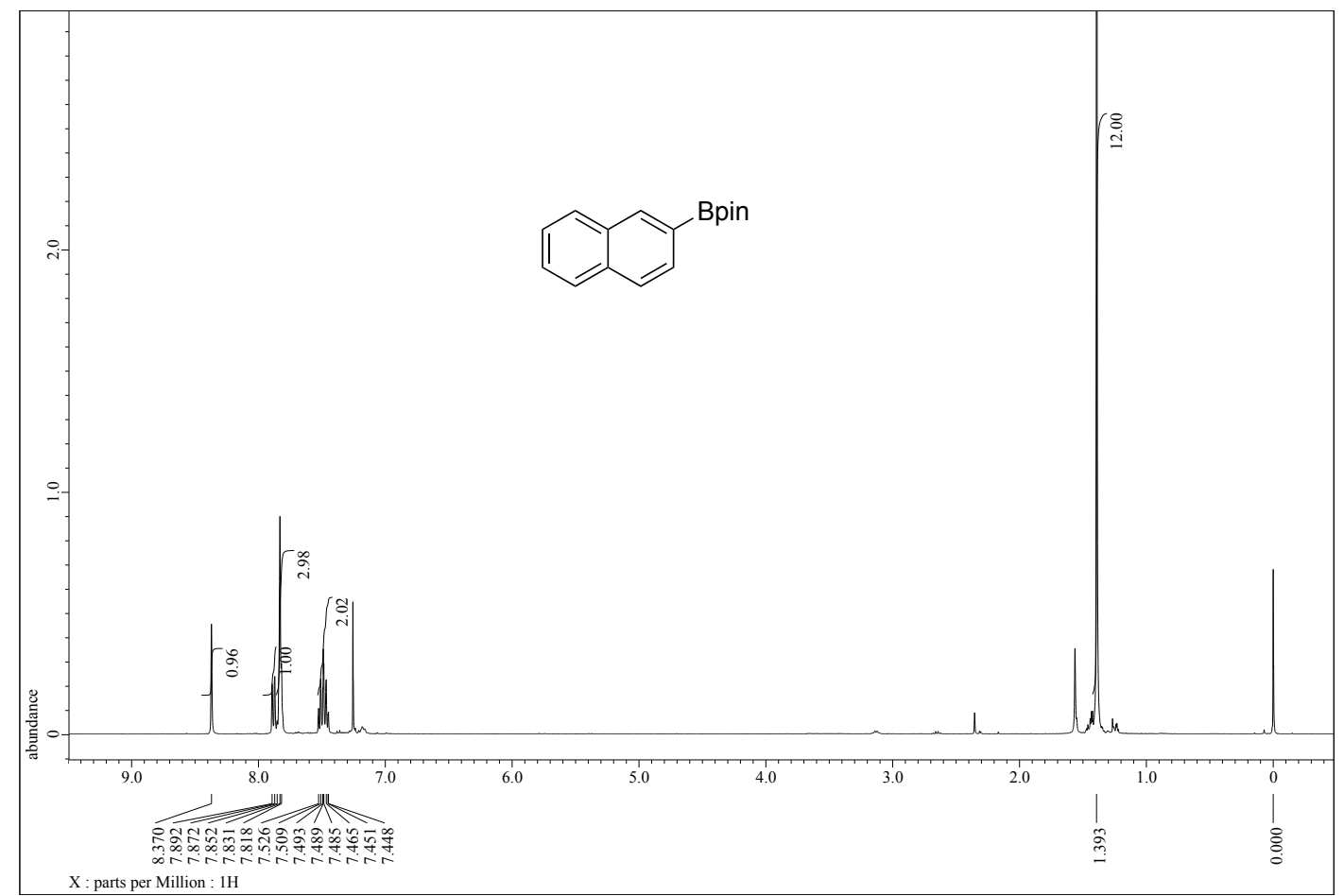

Figure S11. ${ }^{1} \mathrm{H}$ NMR spectrum of $2 \mathrm{c}$ in $\mathrm{CDCl}_{3}$ at $25{ }^{\circ} \mathrm{C}$. 


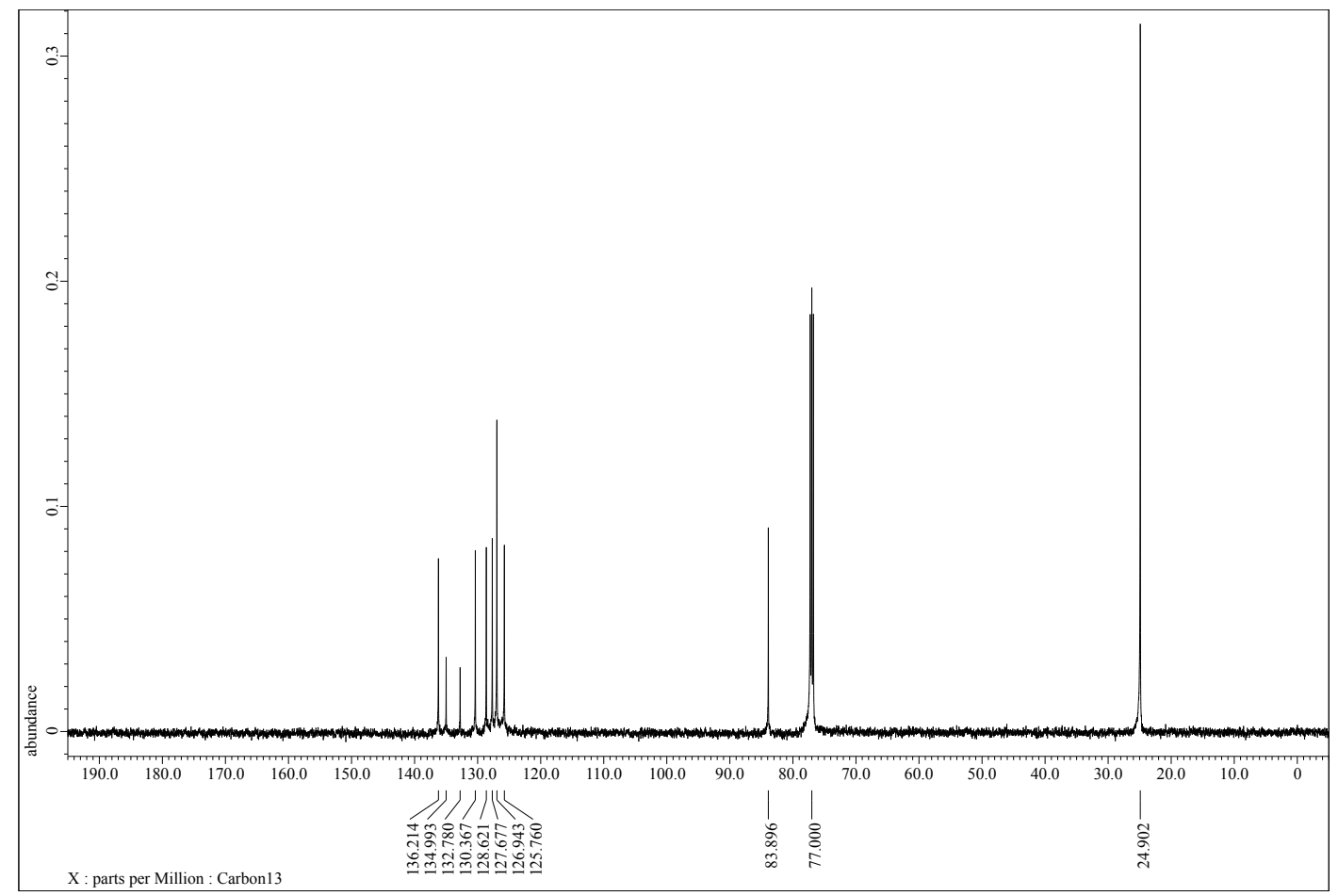

Figure S12. ${ }^{13} \mathrm{C}$ NMR spectrum of $2 \mathrm{c}$ in $\mathrm{CDCl}_{3}$ at $25{ }^{\circ} \mathrm{C}$.

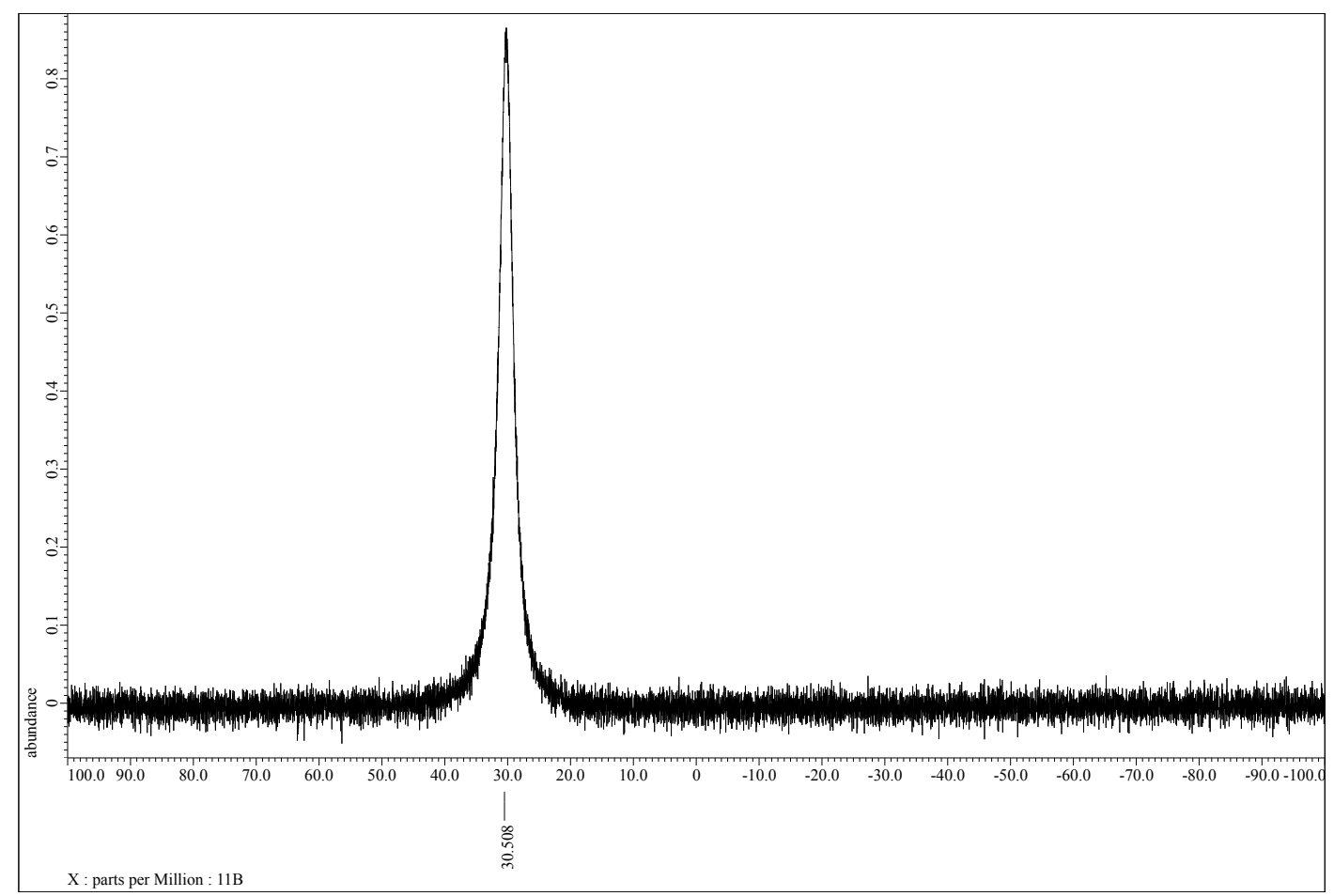

Figure S13. ${ }^{11} \mathrm{~B}$ NMR spectrum of $2 \mathrm{c}$ in $\mathrm{CDCl}_{3}$ at $25{ }^{\circ} \mathrm{C}$. 


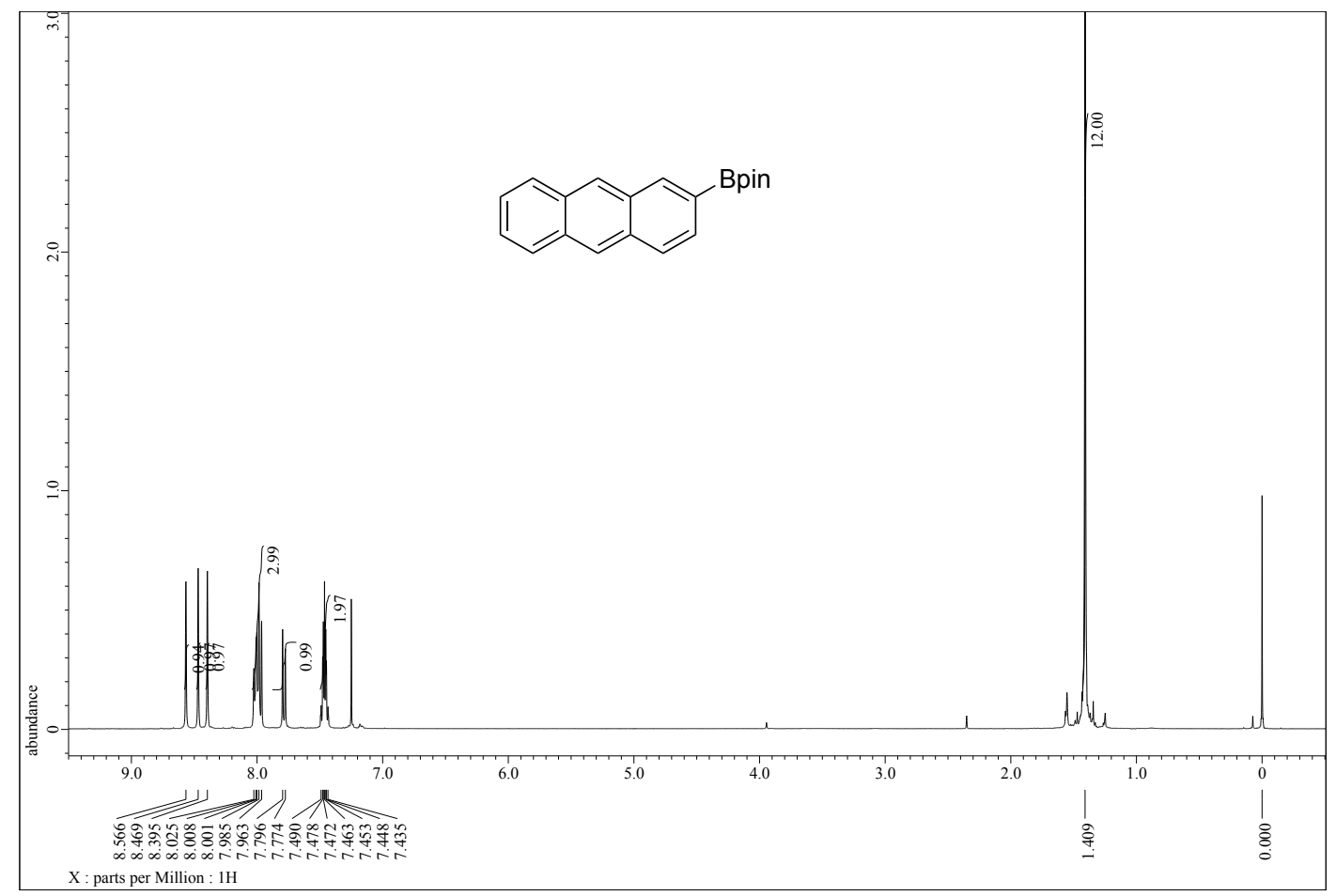

Figure S14. ${ }^{1} \mathrm{H}$ NMR spectrum of $\mathbf{2 d}$ in $\mathrm{CDCl}_{3}$ at $25^{\circ} \mathrm{C}$.

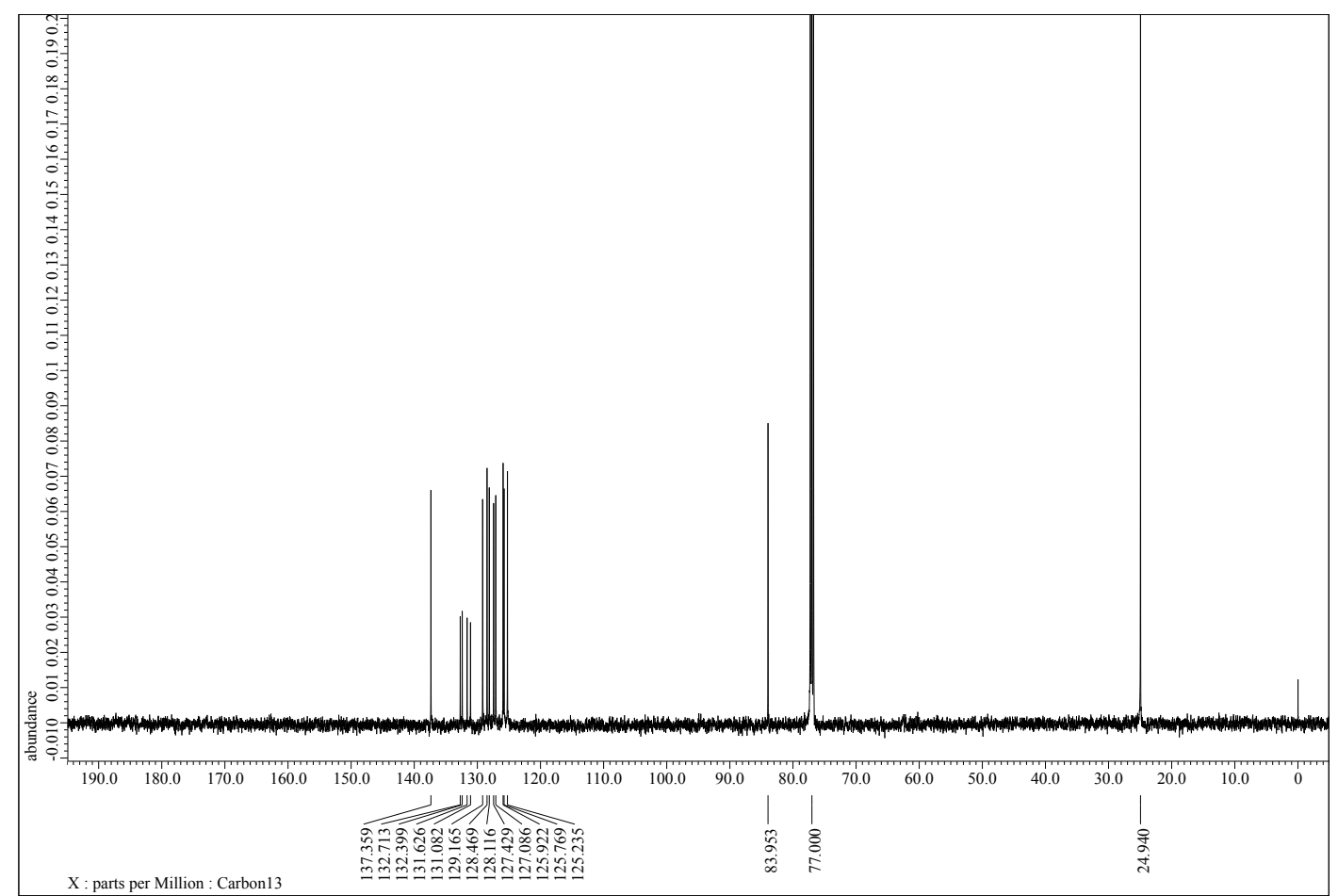

Figure S15. ${ }^{13} \mathrm{C}$ NMR spectrum of $\mathbf{2 d}$ in $\mathrm{CDCl}_{3}$ at $25{ }^{\circ} \mathrm{C}$. 


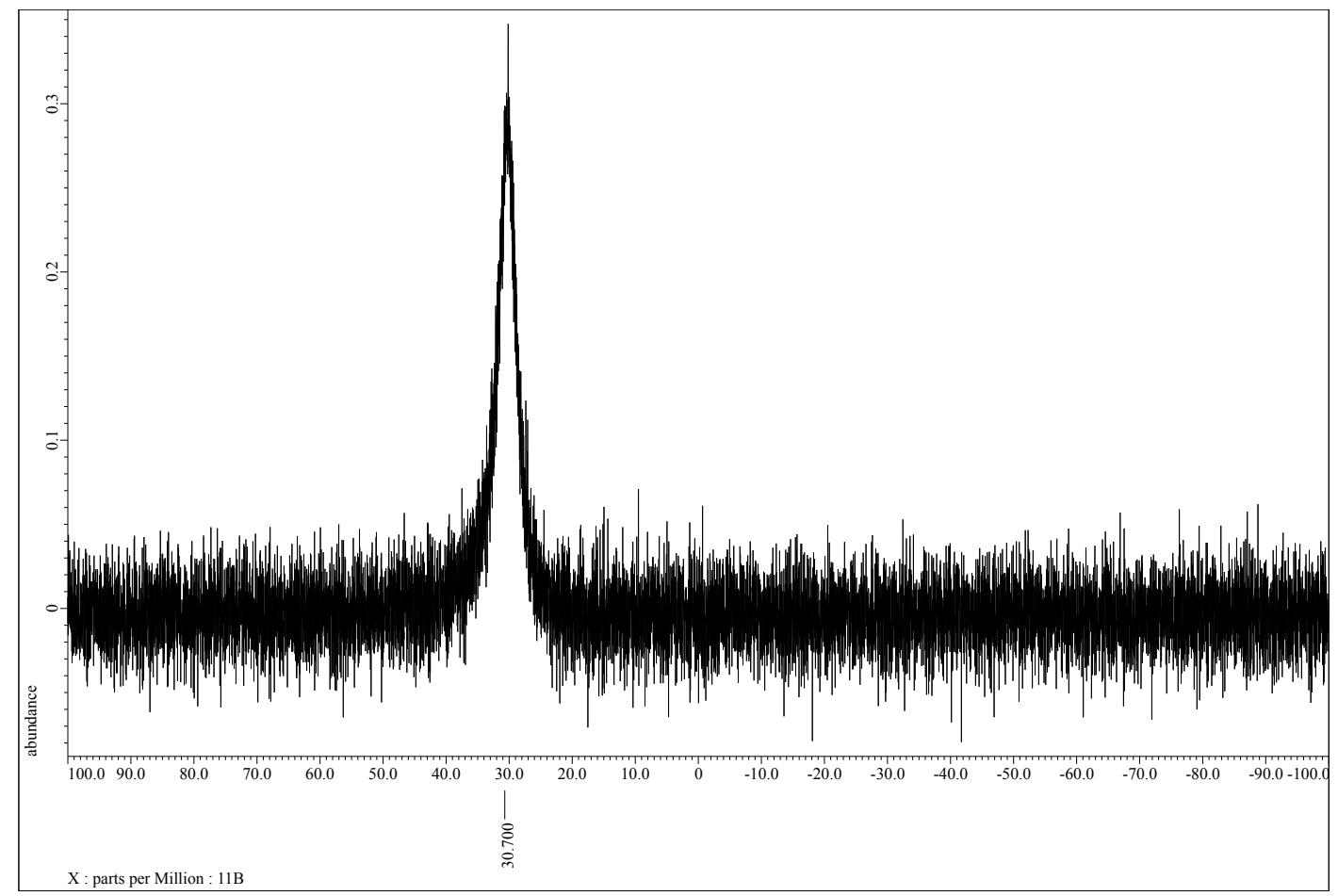

Figure S16. ${ }^{11} \mathrm{~B}$ NMR spectrum of $2 \mathbf{d}$ in $\mathrm{CDCl}_{3}$ at $25^{\circ} \mathrm{C}$.

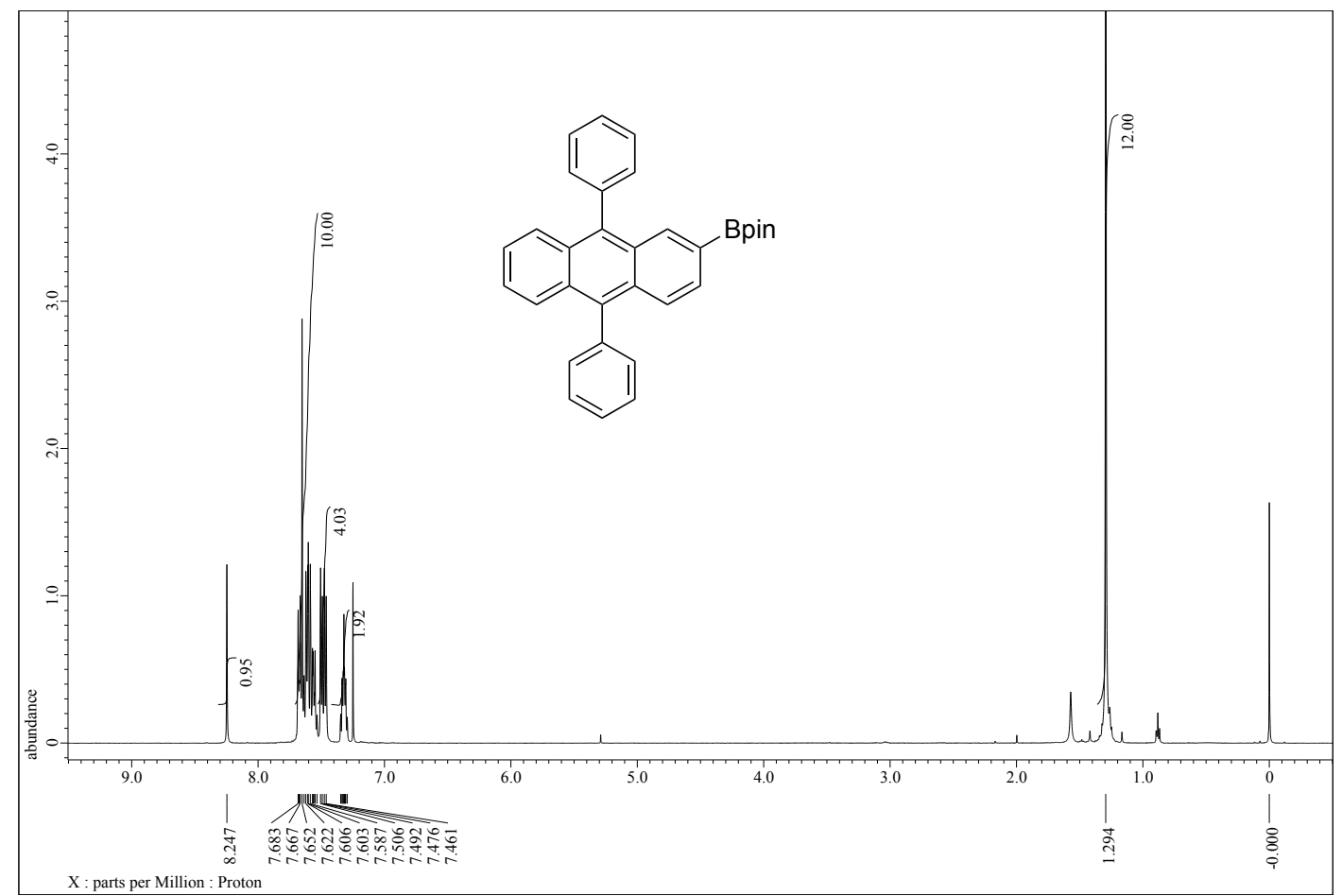

Figure S17. ${ }^{1} \mathrm{H}$ NMR spectrum of $2 \mathrm{e}$ in $\mathrm{CDCl}_{3}$ at $25^{\circ} \mathrm{C}$. 


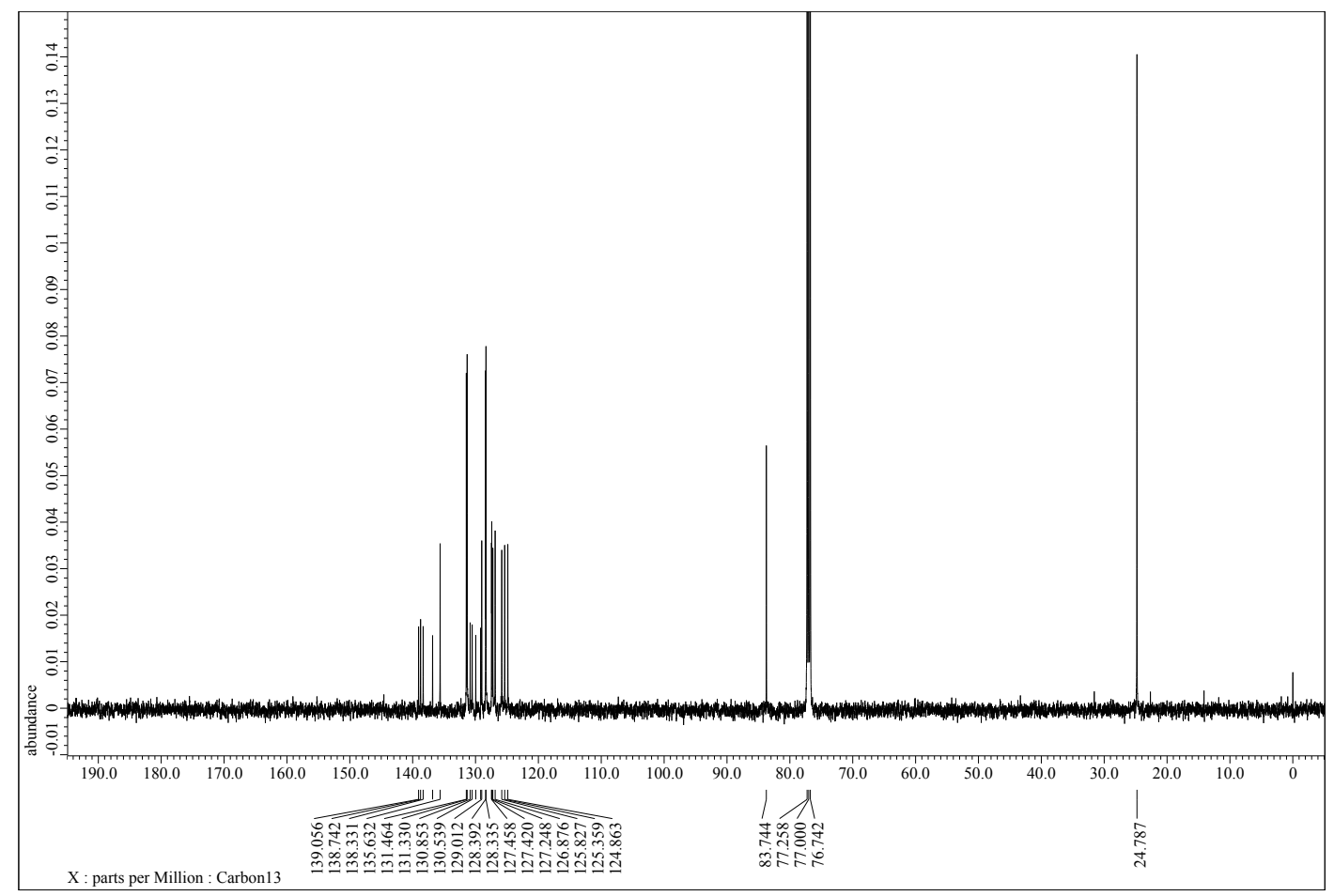

Figure S18. ${ }^{13} \mathrm{C}$ NMR spectrum of $2 \mathrm{e}$ in $\mathrm{CDCl}_{3}$ at $25{ }^{\circ} \mathrm{C}$.

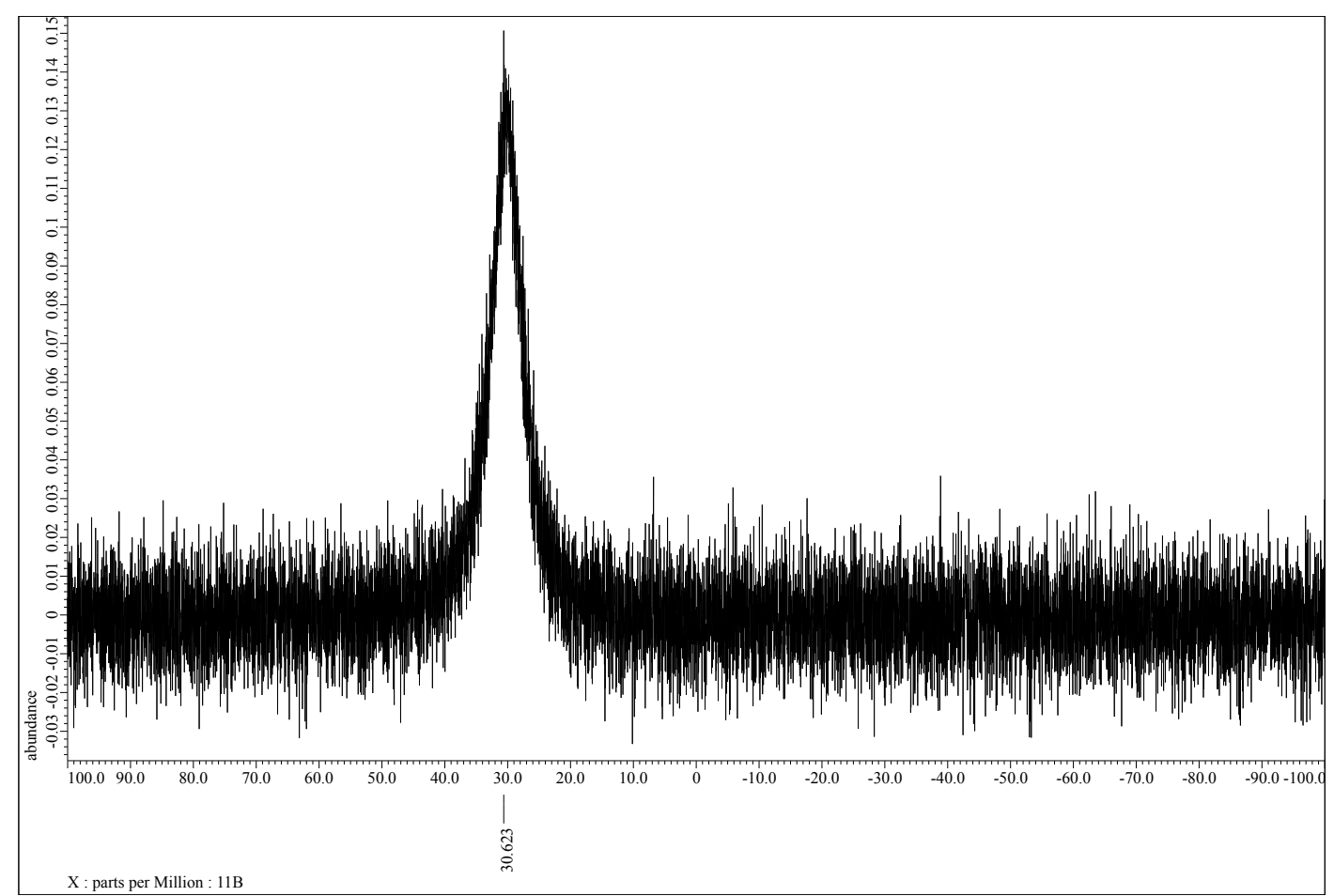

Figure S19. ${ }^{11} \mathrm{~B}$ NMR spectrum of $2 \mathbf{e}$ in $\mathrm{CDCl}_{3}$ at $25{ }^{\circ} \mathrm{C}$. 


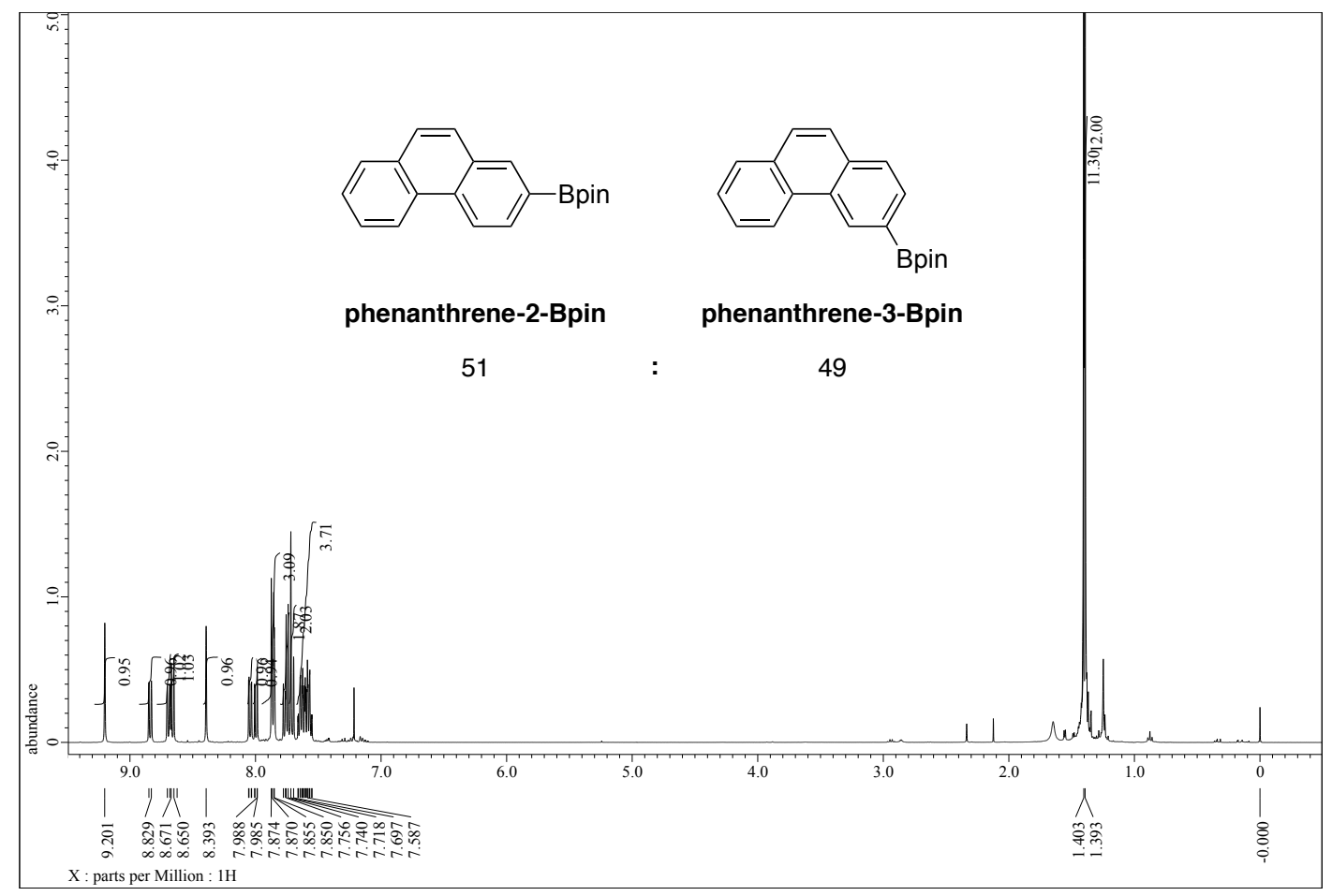

Figure S20. ${ }^{1} \mathrm{H}$ NMR spectrum of $\mathbf{2} \mathbf{f}$ in $\mathrm{CDCl}_{3}$ at $25{ }^{\circ} \mathrm{C}$.

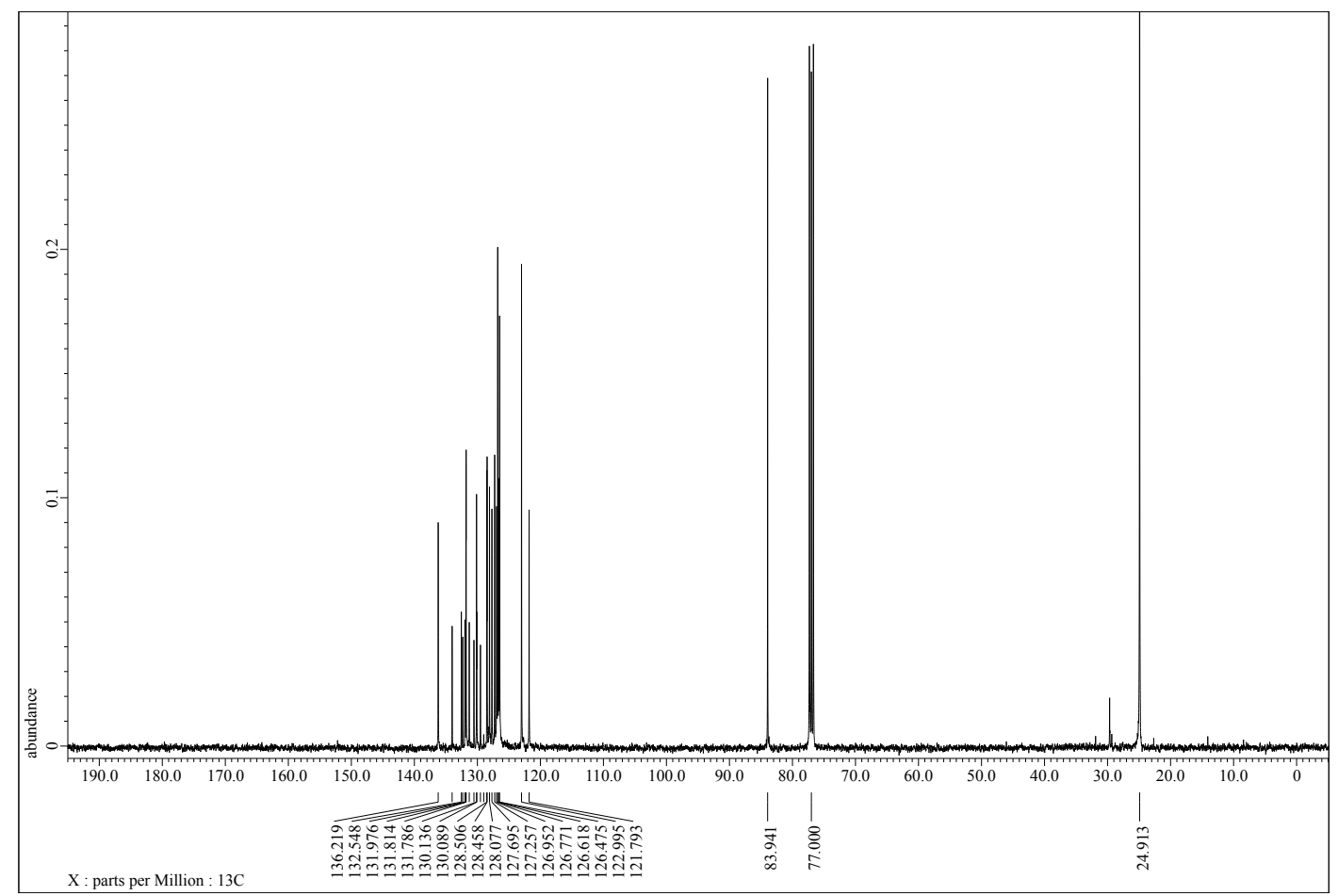

Figure S21. ${ }^{13} \mathrm{C}$ NMR spectrum of $\mathbf{2 f}$ in $\mathrm{CDCl}_{3}$ at $25{ }^{\circ} \mathrm{C}$. 


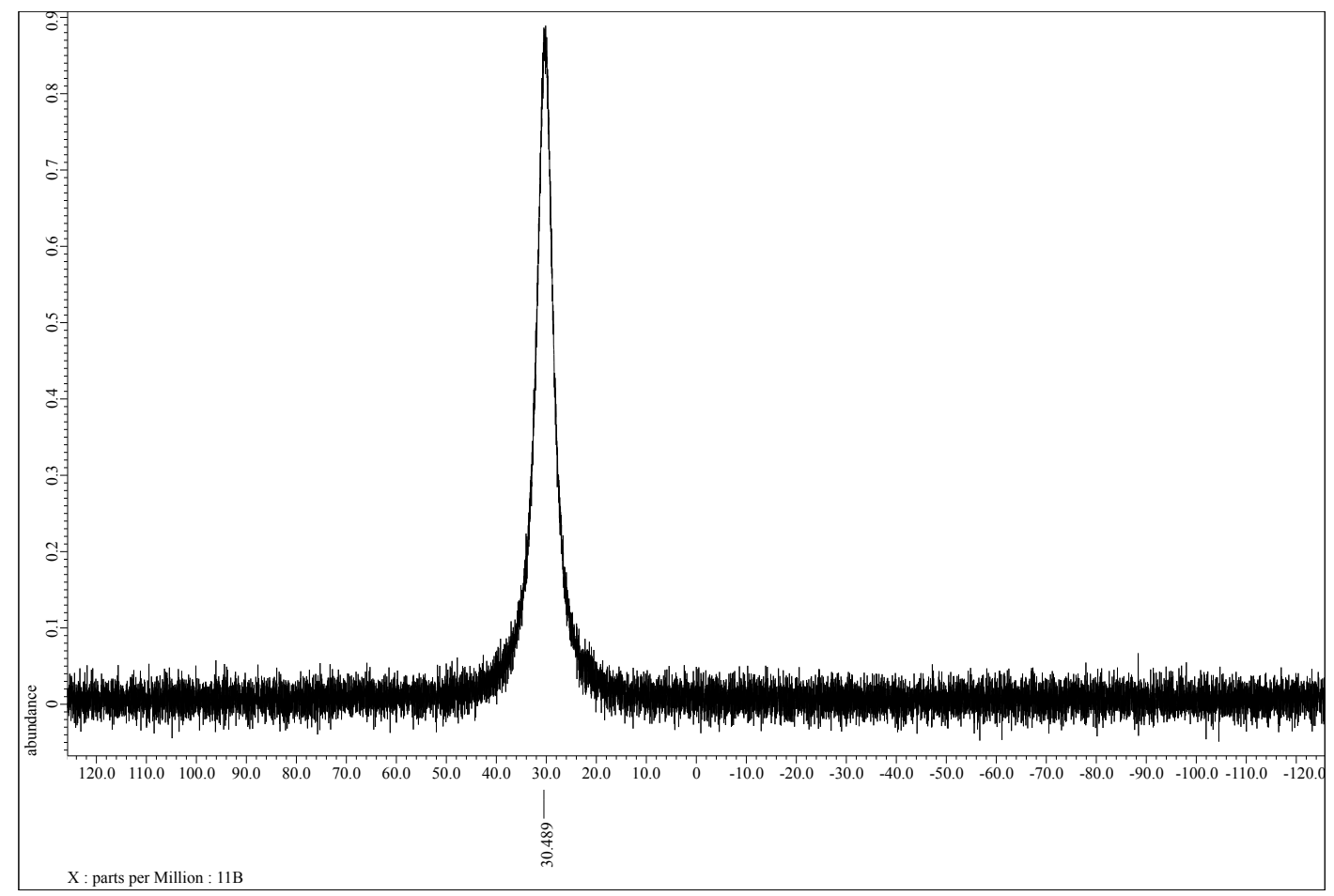

Figure S22. ${ }^{11} \mathrm{~B}$ NMR spectrum of $\mathbf{2 f}$ in $\mathrm{CDCl}_{3}$ at $25^{\circ} \mathrm{C}$.

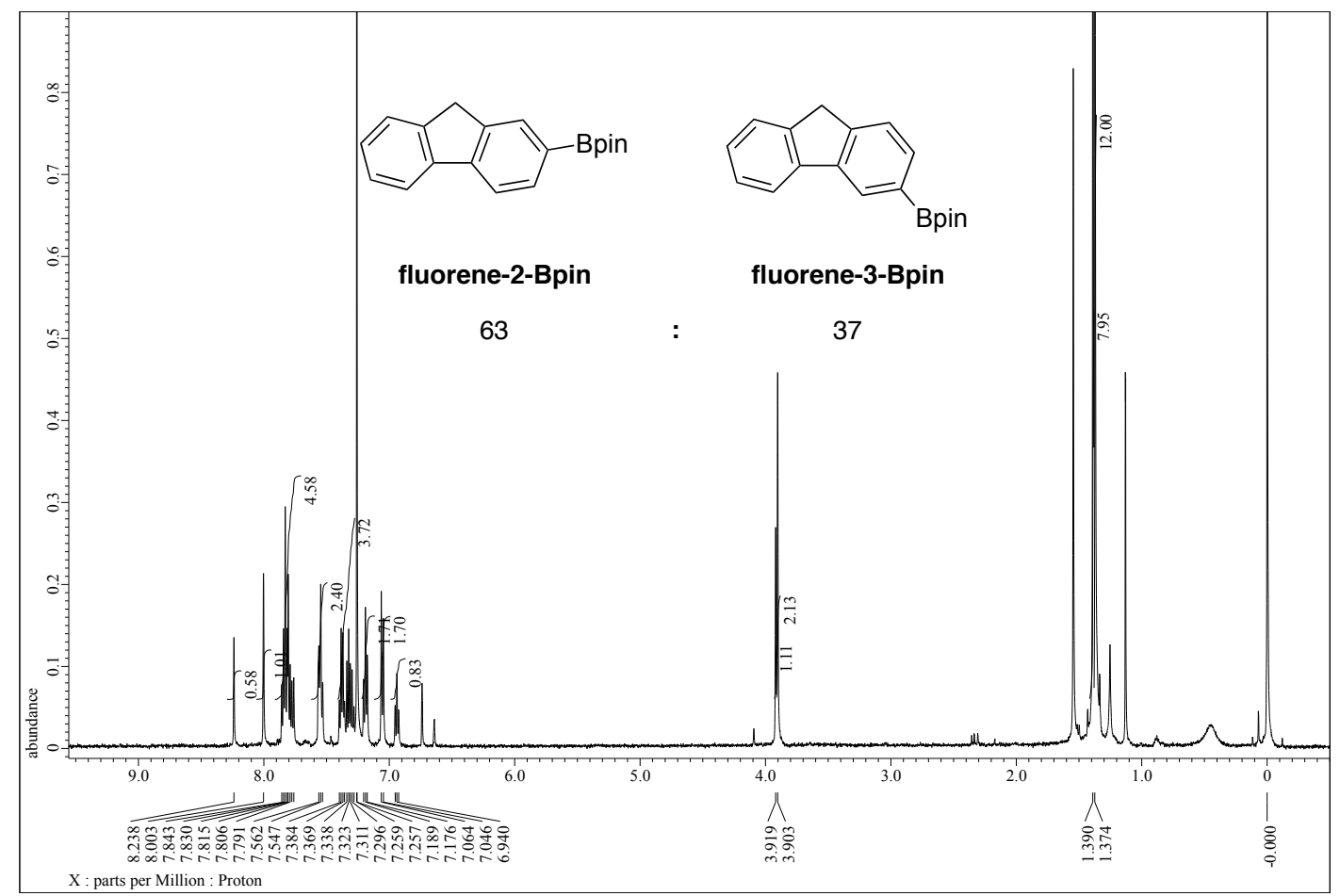

Figure S23. ${ }^{1} \mathrm{H}$ NMR spectrum of $\mathbf{2 g}$ in $\mathrm{CDCl}_{3}$ at $25{ }^{\circ} \mathrm{C}$. 


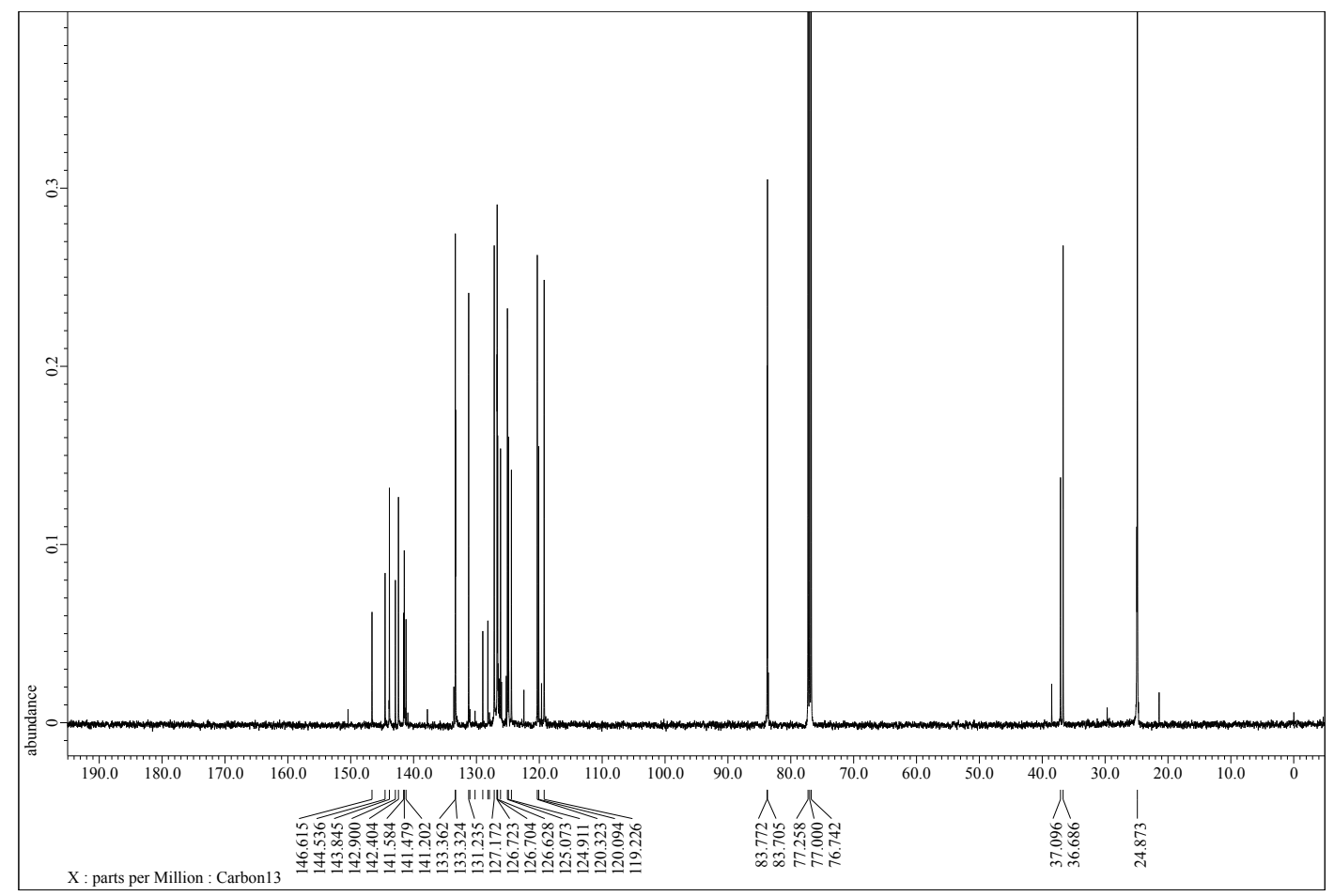

Figure S24. ${ }^{13} \mathrm{C}$ NMR spectrum of $2 \mathrm{~g}$ in $\mathrm{CDCl}_{3}$ at $25{ }^{\circ} \mathrm{C}$.

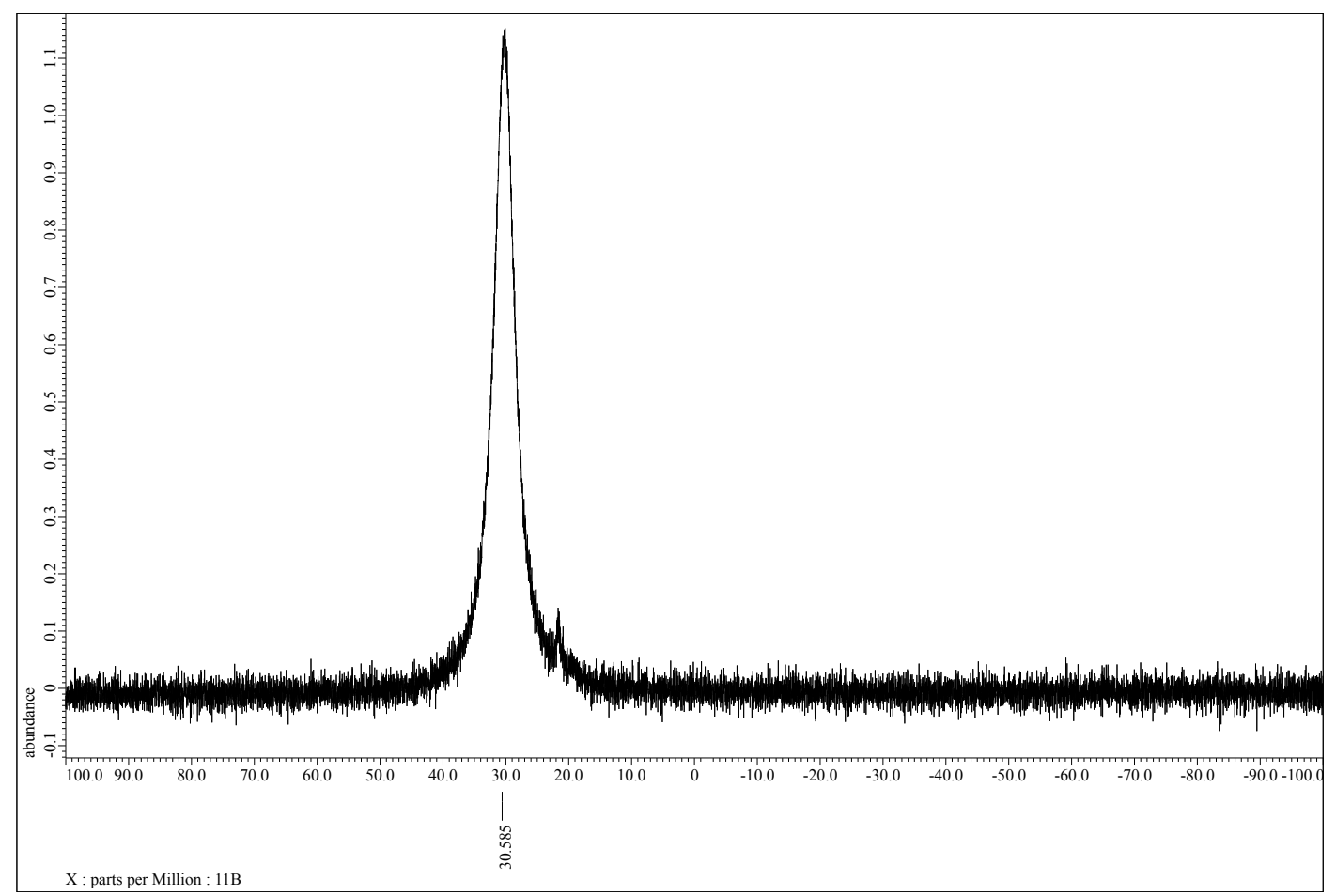

Figure S25. ${ }^{11} \mathrm{~B}$ NMR spectrum of $\mathbf{2 g}$ in $\mathrm{CDCl}_{3}$ at $25^{\circ} \mathrm{C}$. 


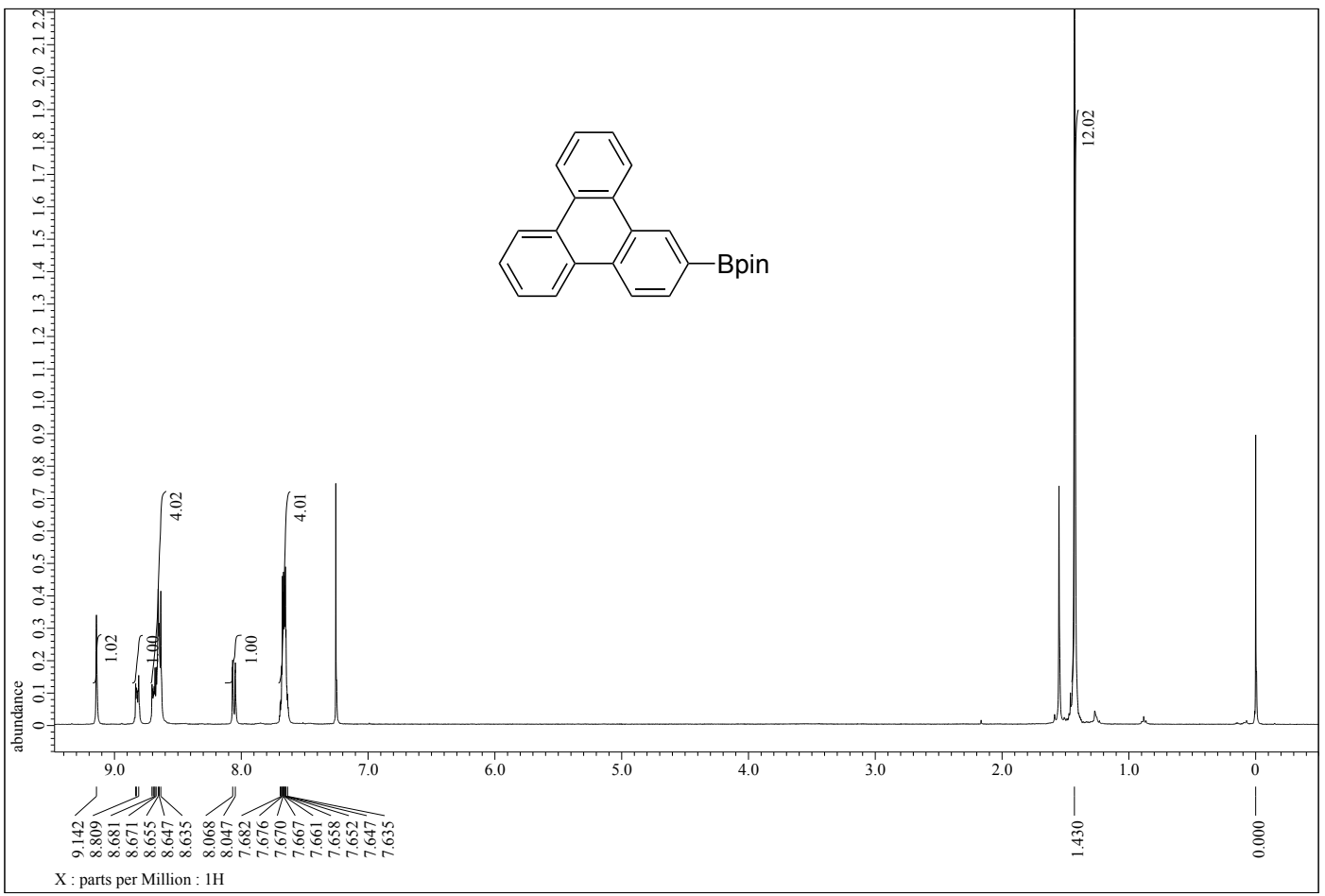

Figure S26. ${ }^{1} \mathrm{H}$ NMR spectrum of $\mathbf{2 h}$ in $\mathrm{CDCl}_{3}$ at $25{ }^{\circ} \mathrm{C}$.

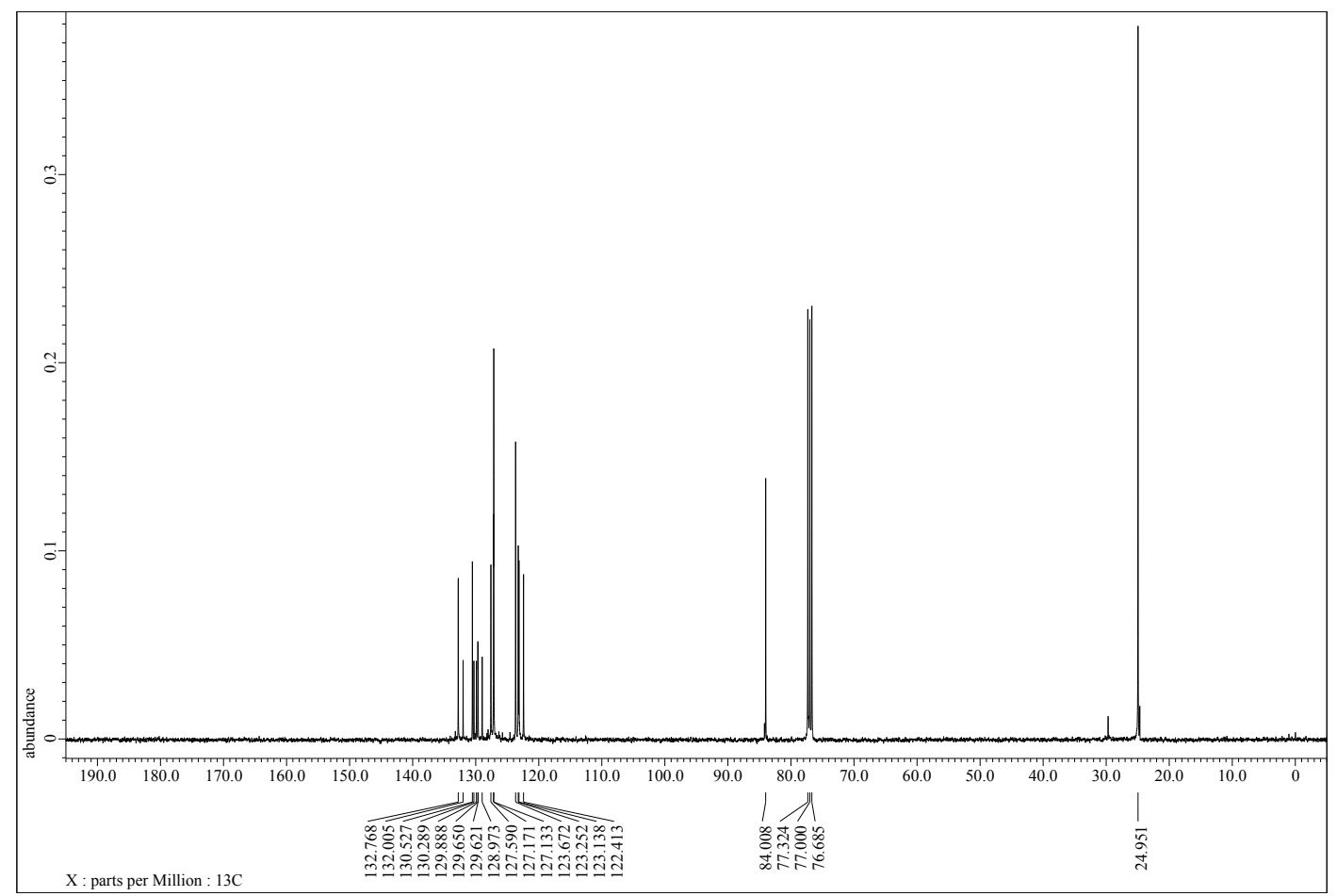

Figure S27. ${ }^{13} \mathrm{C}$ NMR spectrum of $\mathbf{2 h}$ in $\mathrm{CDCl}_{3}$ at $25{ }^{\circ} \mathrm{C}$. 


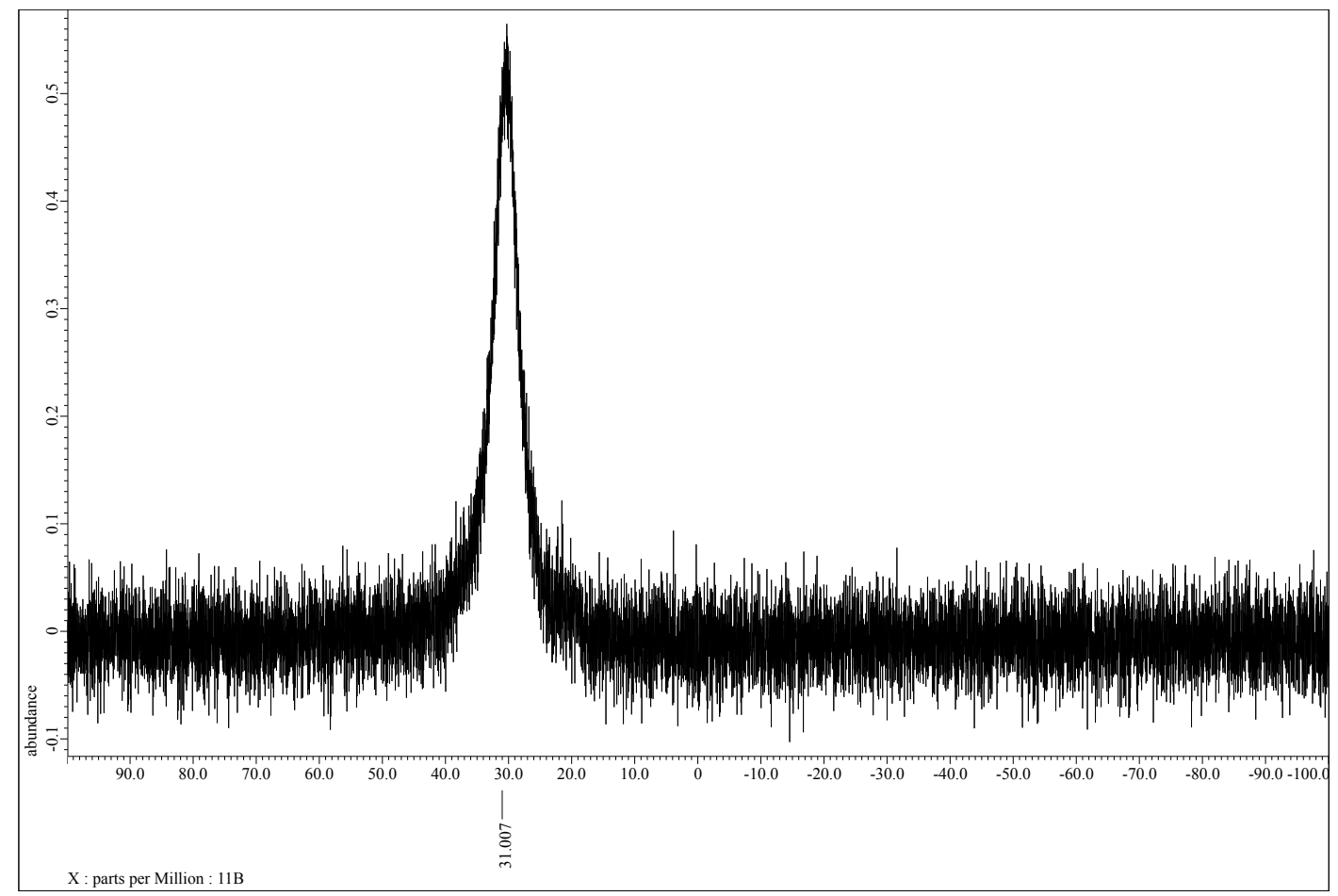

Figure S28. ${ }^{11} \mathrm{~B}$ NMR spectrum of $\mathbf{2 h}$ in $\mathrm{CDCl}_{3}$ at $25{ }^{\circ} \mathrm{C}$.

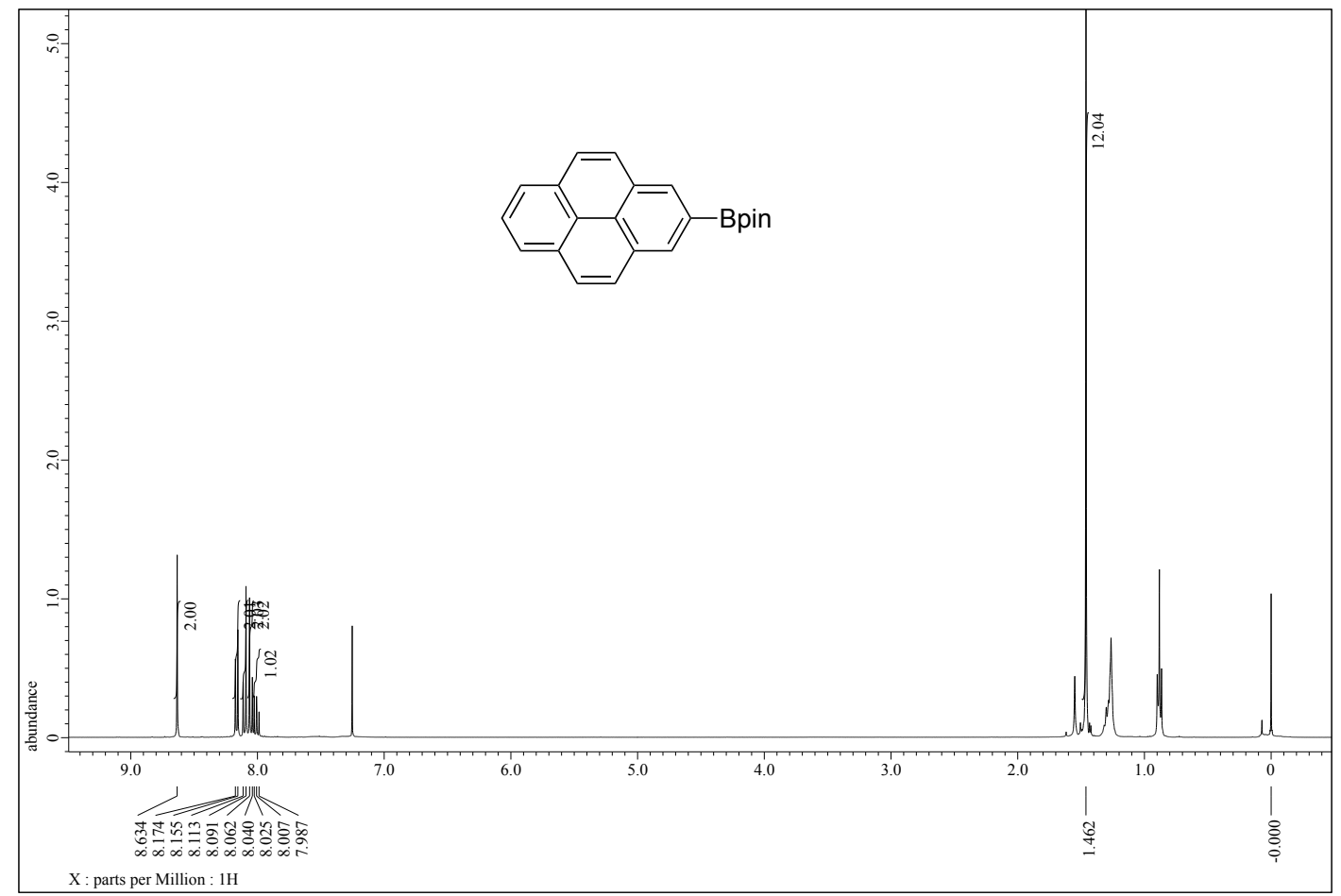

Figure S29. ${ }^{1} \mathrm{H}$ NMR spectrum of $2 \mathbf{i}$ in $\mathrm{CDCl}_{3}$ at $25{ }^{\circ} \mathrm{C}$. 


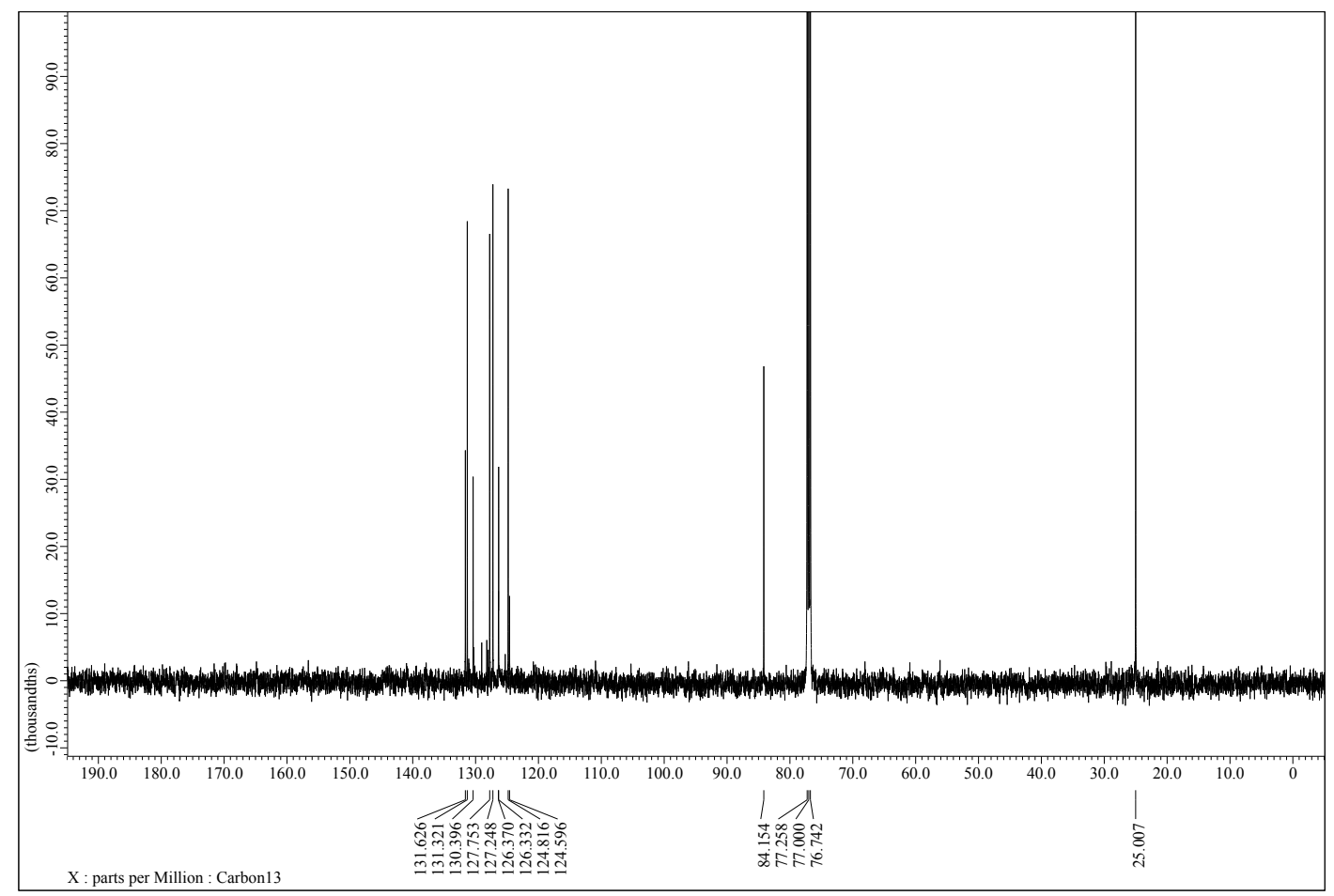

Figure S30. ${ }^{13} \mathrm{C}$ NMR spectrum of $2 \mathbf{i}$ in $\mathrm{CDCl}_{3}$ at $25{ }^{\circ} \mathrm{C}$.

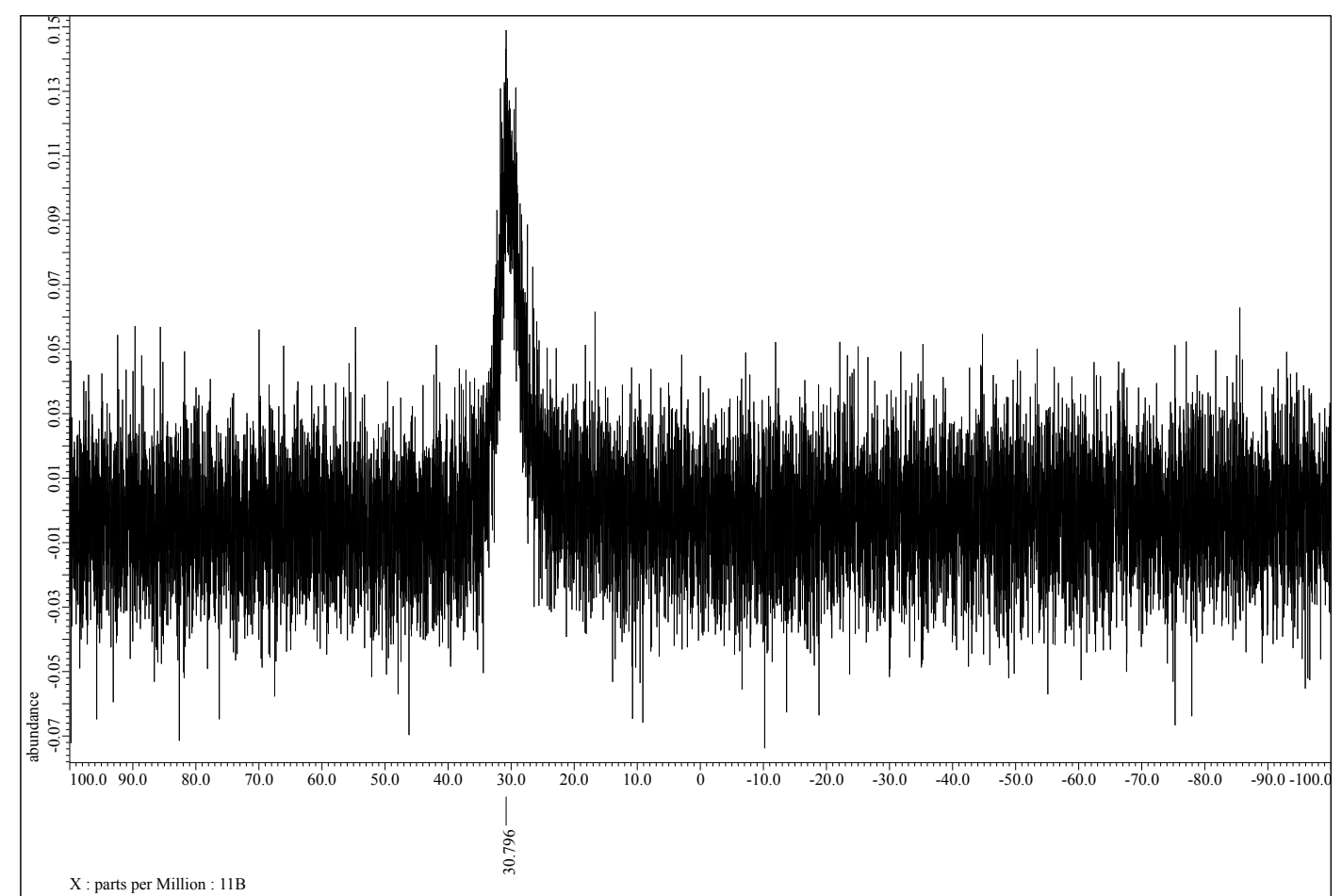

Figure S31. ${ }^{11} \mathrm{~B}$ NMR spectrum of $2 \mathbf{i}$ in $\mathrm{CDCl}_{3}$ at $25{ }^{\circ} \mathrm{C}$. 


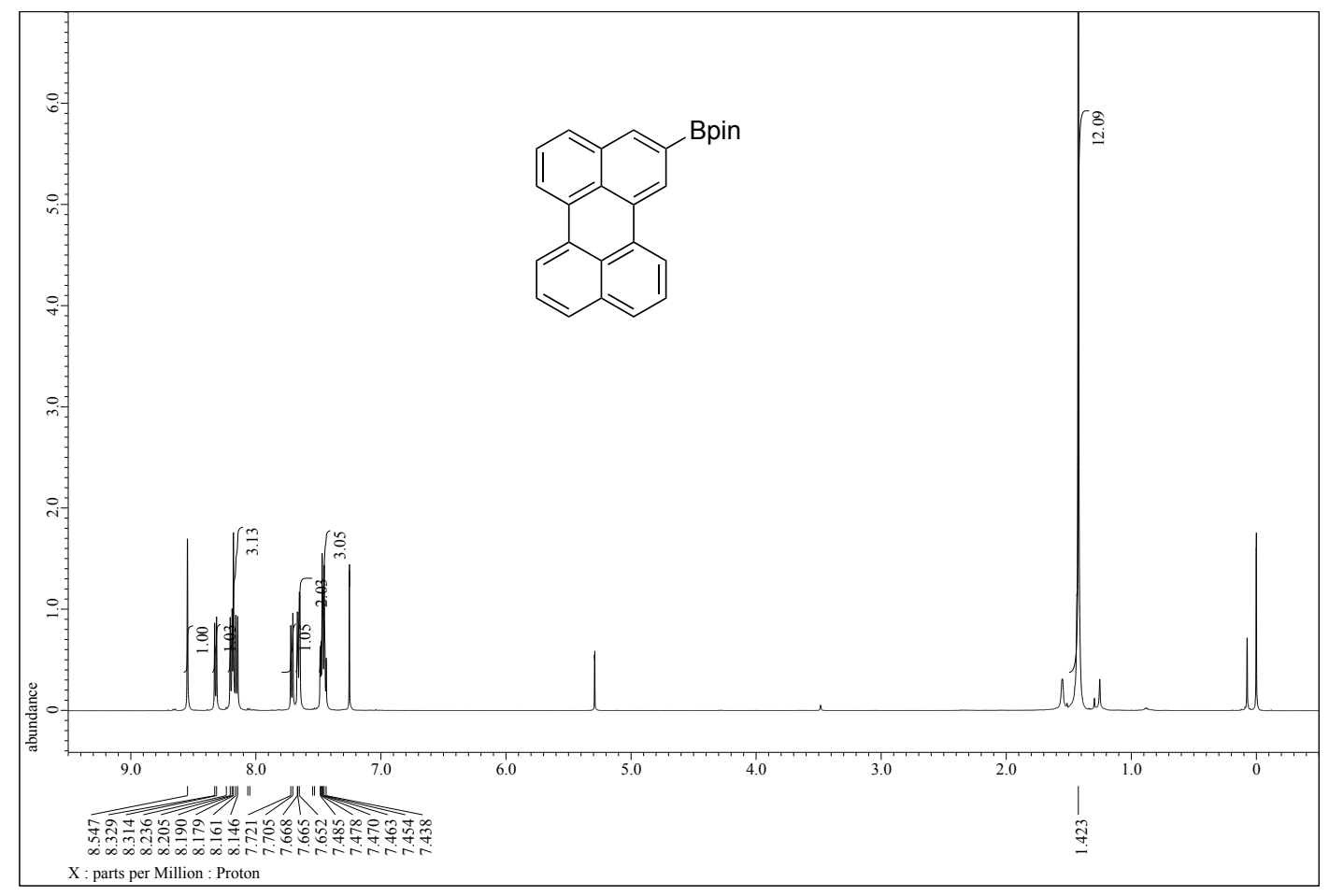

Figure S32. ${ }^{1} \mathrm{H}$ NMR spectrum of $\mathbf{2} \mathbf{j}$ in $\mathrm{CDCl}_{3}$ at $25{ }^{\circ} \mathrm{C}$.

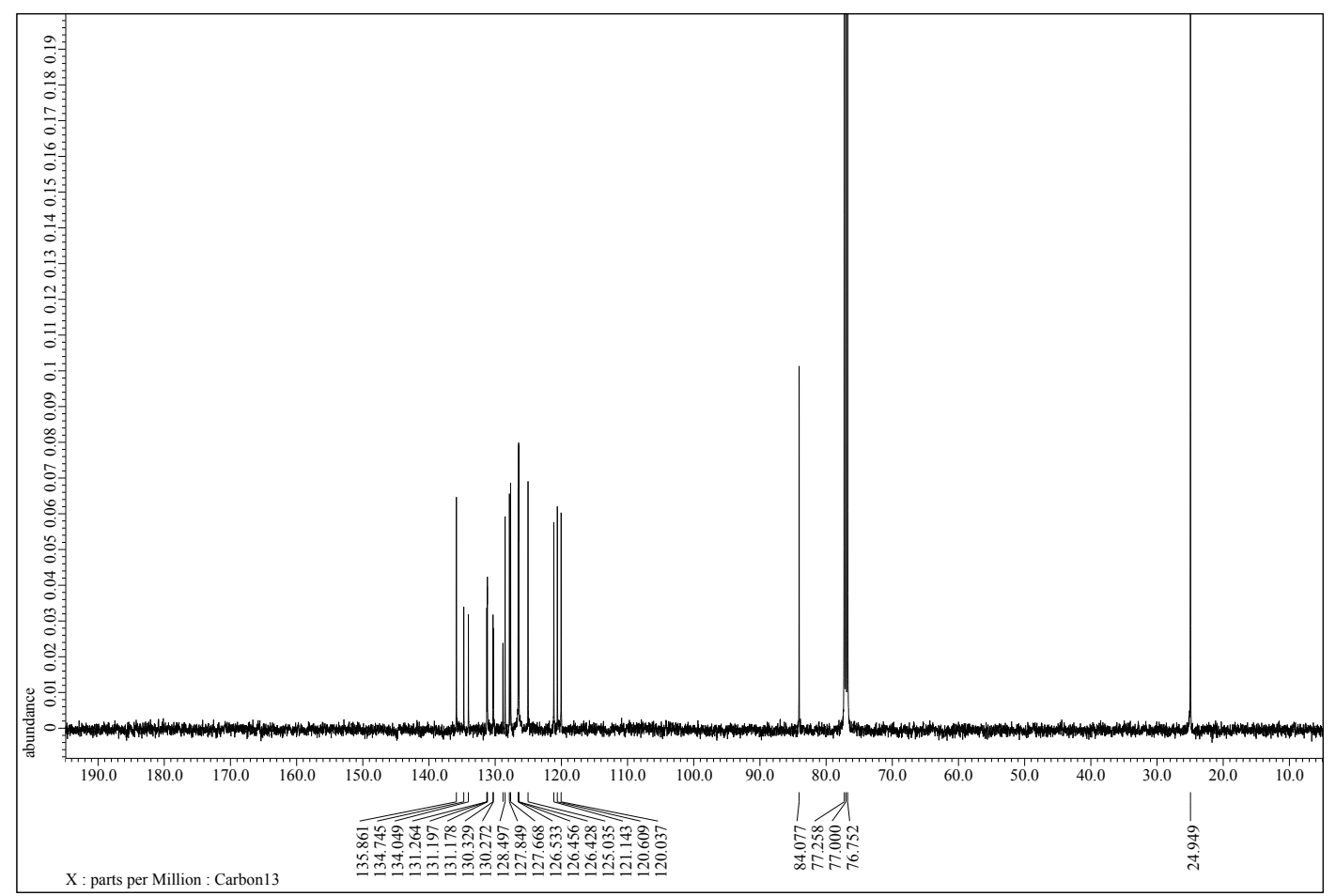

Figure S33. ${ }^{13} \mathrm{C}$ NMR spectrum of $2 \mathbf{j}$ in $\mathrm{CDCl}_{3}$ at $25{ }^{\circ} \mathrm{C}$. 


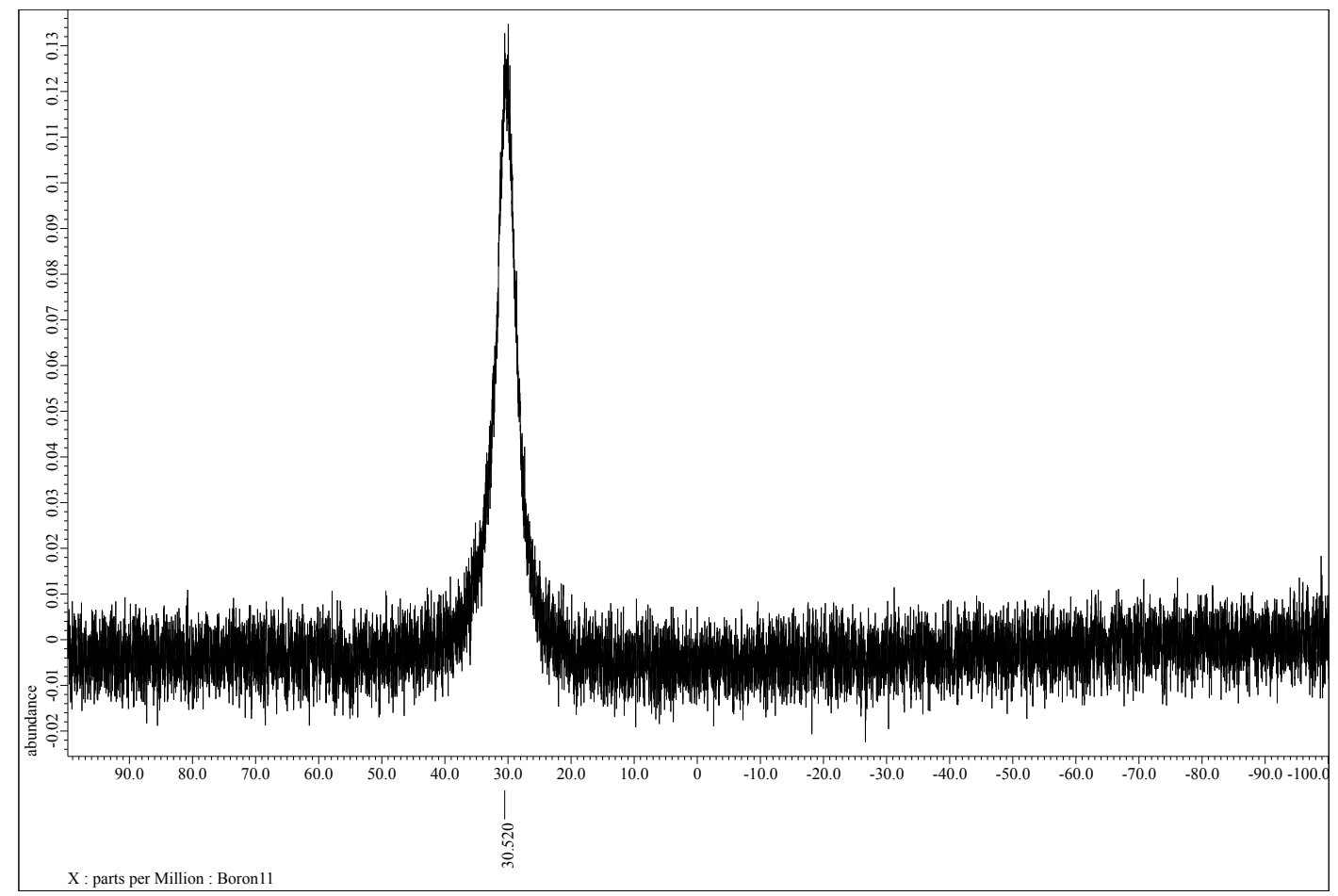

Figure S34. ${ }^{11} \mathrm{~B}$ NMR spectrum of $\mathbf{2} \mathbf{j}$ in $\mathrm{CDCl}_{3}$ at $25{ }^{\circ} \mathrm{C}$.

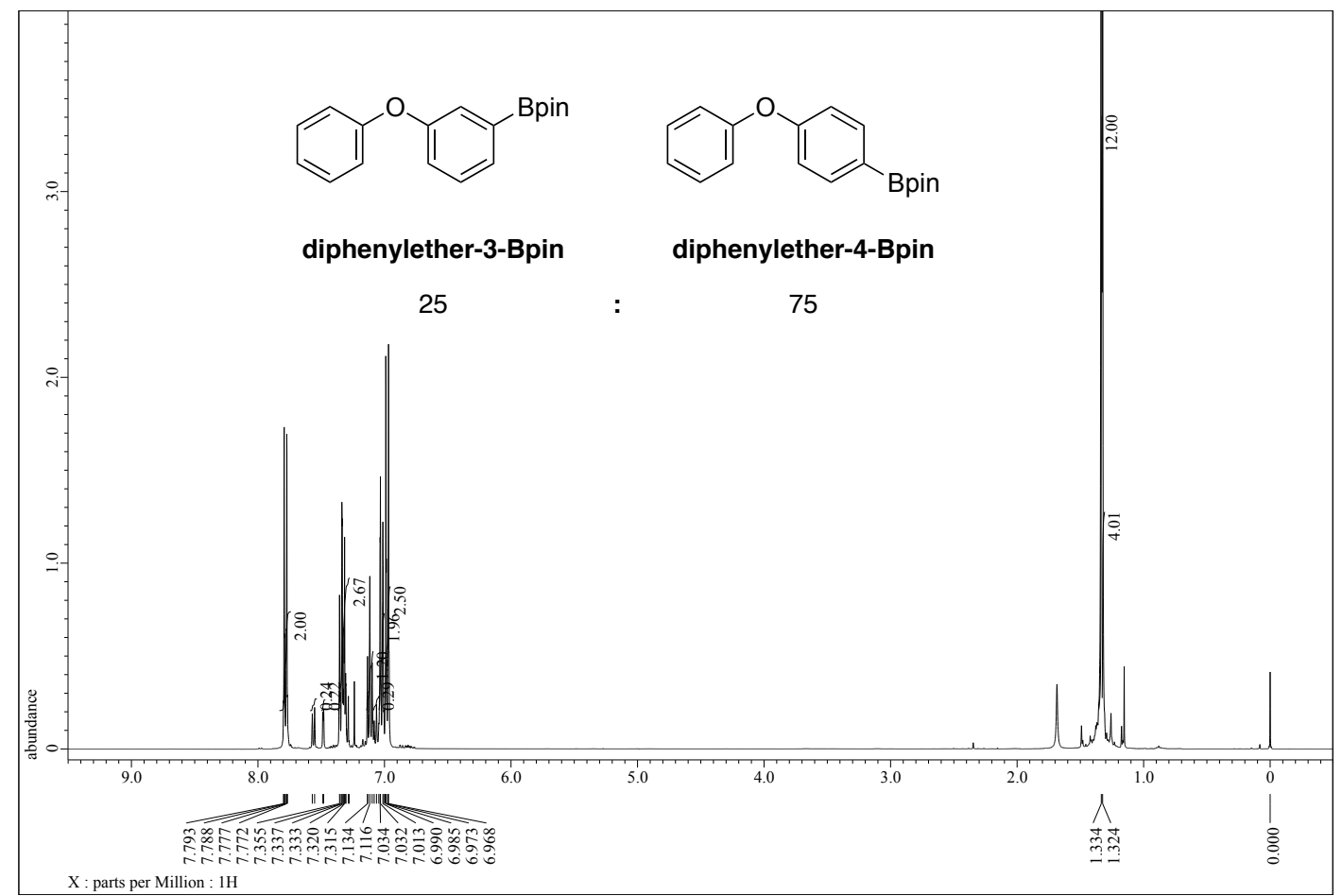

Figure S35. ${ }^{1} \mathrm{H}$ NMR spectrum of $\mathbf{2 k}$ in $\mathrm{CDCl}_{3}$ at $25{ }^{\circ} \mathrm{C}$. 


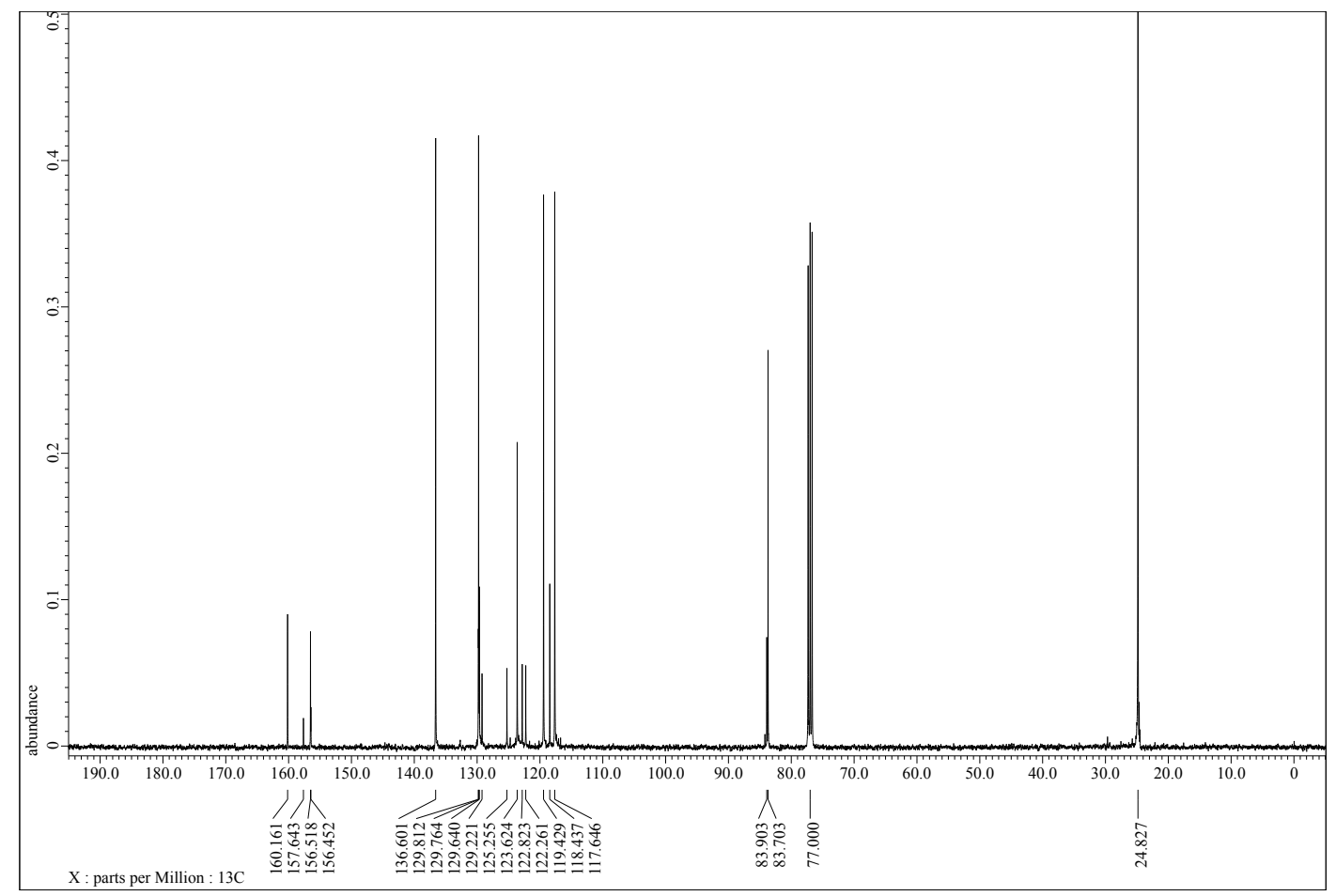

Figure S36. ${ }^{13} \mathrm{C}$ NMR spectrum of $\mathbf{2 k}$ in $\mathrm{CDCl}_{3}$ at $25{ }^{\circ} \mathrm{C}$.

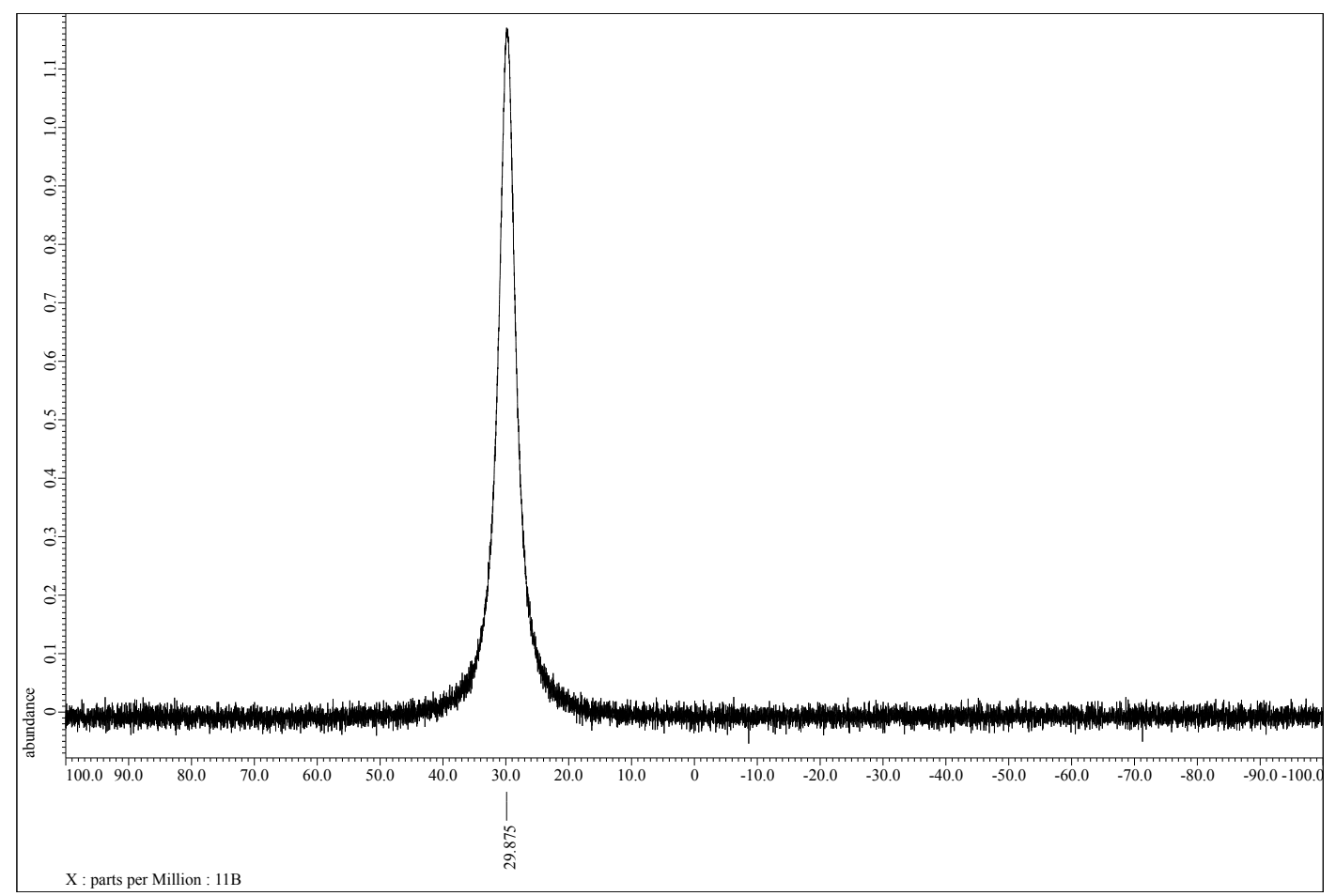

Figure S37. ${ }^{11} \mathrm{~B}$ NMR spectrum of $\mathbf{2} \mathbf{k}$ in $\mathrm{CDCl}_{3}$ at $25^{\circ} \mathrm{C}$. 


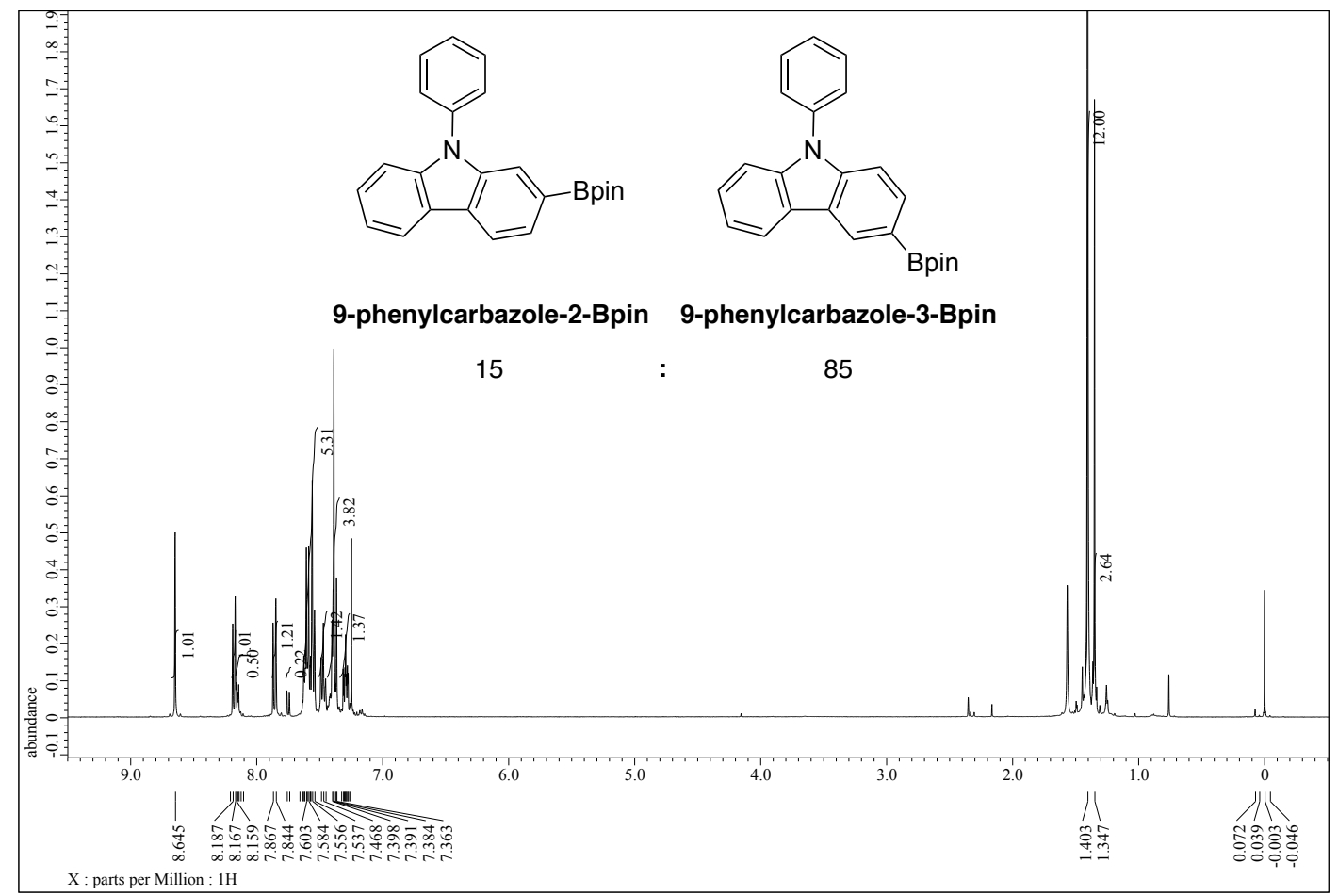

Figure S38. ${ }^{1} \mathrm{H}$ NMR spectrum of $2 \mathbf{l}$ in $\mathrm{CDCl}_{3}$ at $25{ }^{\circ} \mathrm{C}$.

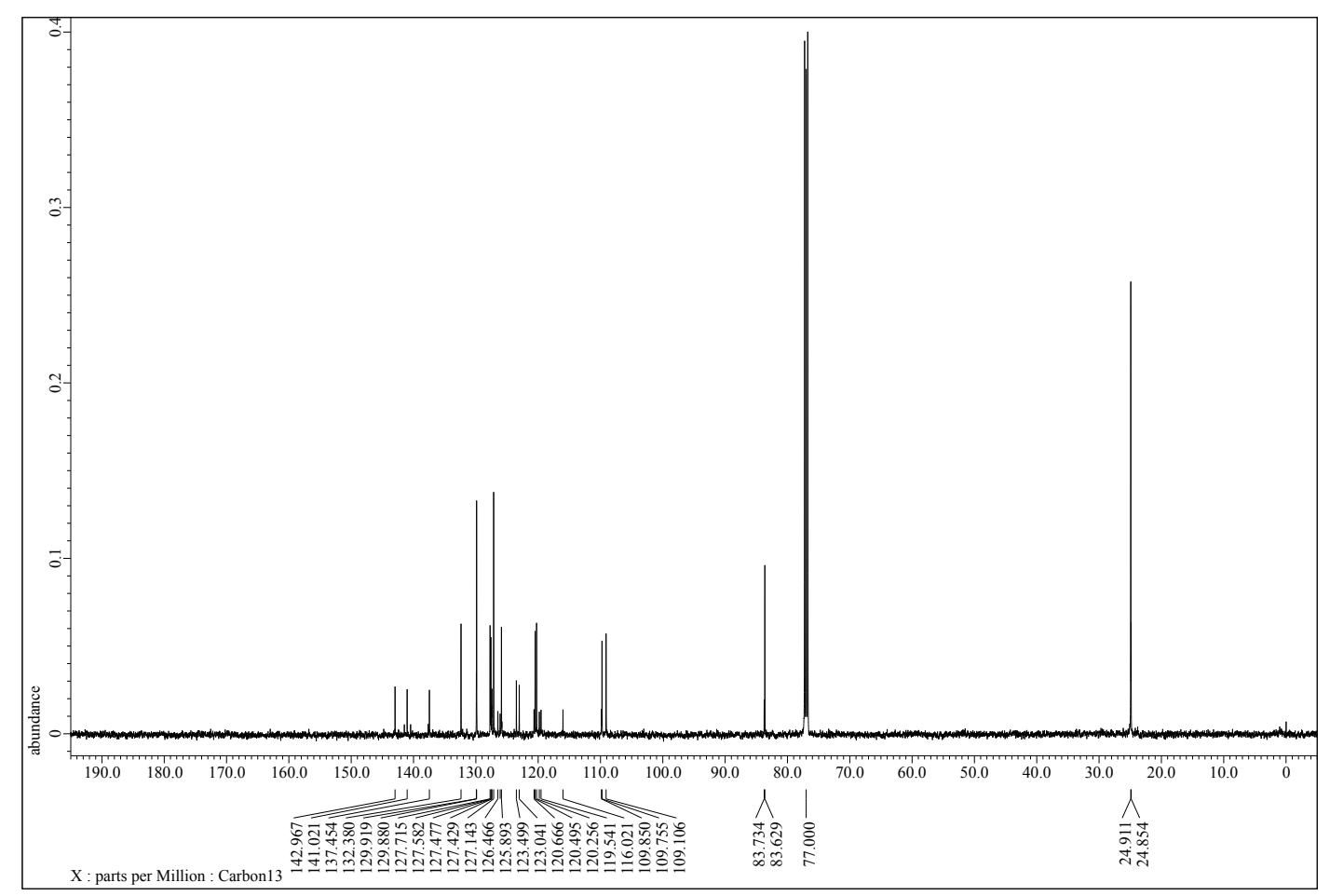

Figure S39. ${ }^{13} \mathrm{C}$ NMR spectrum of $2 \mathbf{l}$ in $\mathrm{CDCl}_{3}$ at $25{ }^{\circ} \mathrm{C}$. 


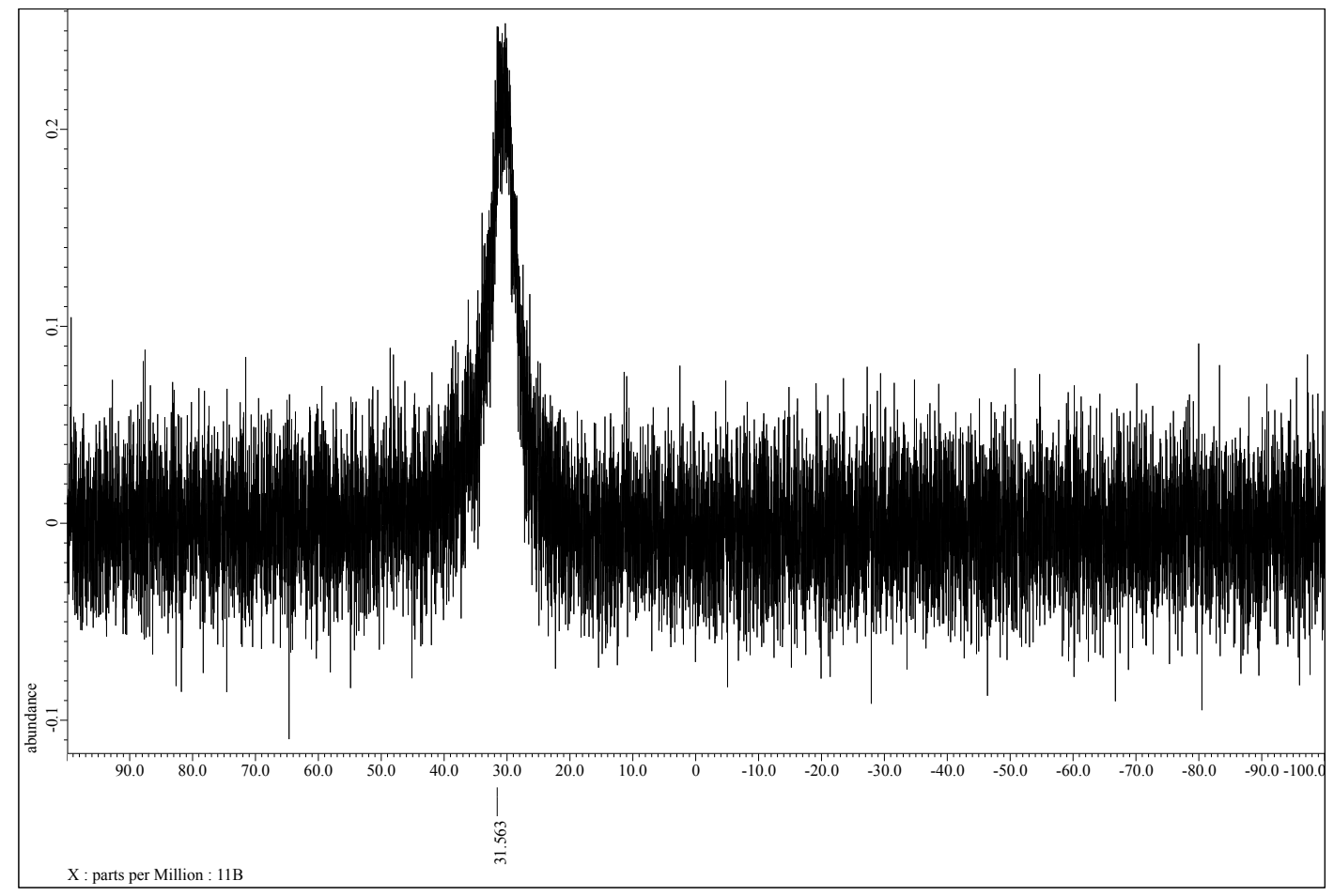

Figure S40. ${ }^{11} \mathrm{~B}$ NMR spectrum of 21 in $\mathrm{CDCl}_{3}$ at $25{ }^{\circ} \mathrm{C}$.

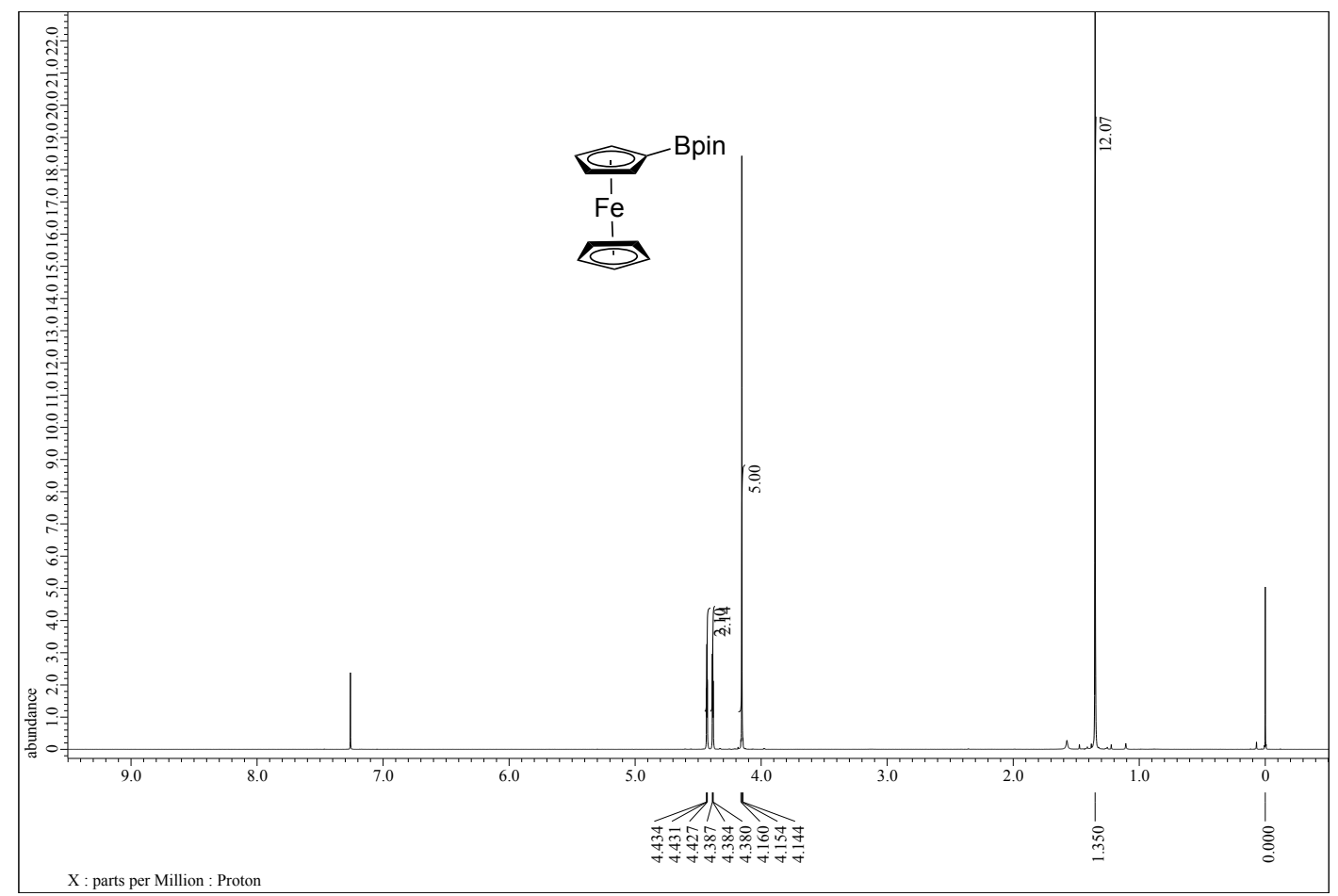

Figure S41. ${ }^{1} \mathrm{H}$ NMR spectrum of $\mathbf{2 m}$ in $\mathrm{CDCl}_{3}$ at $25{ }^{\circ} \mathrm{C}$. 


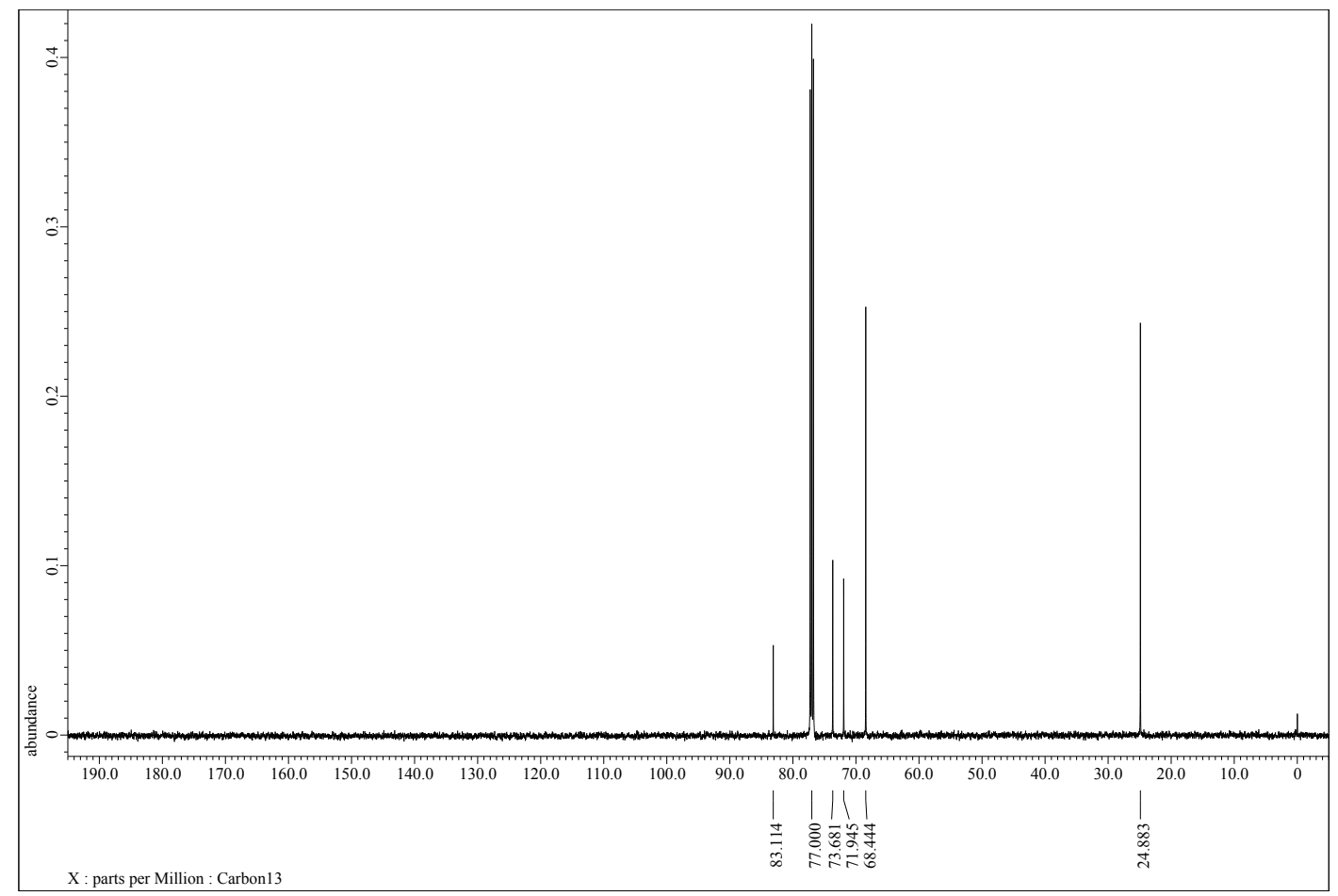

Figure S42. ${ }^{13} \mathrm{C}$ NMR spectrum of $\mathbf{2 m}$ in $\mathrm{CDCl}_{3}$ at $25{ }^{\circ} \mathrm{C}$.

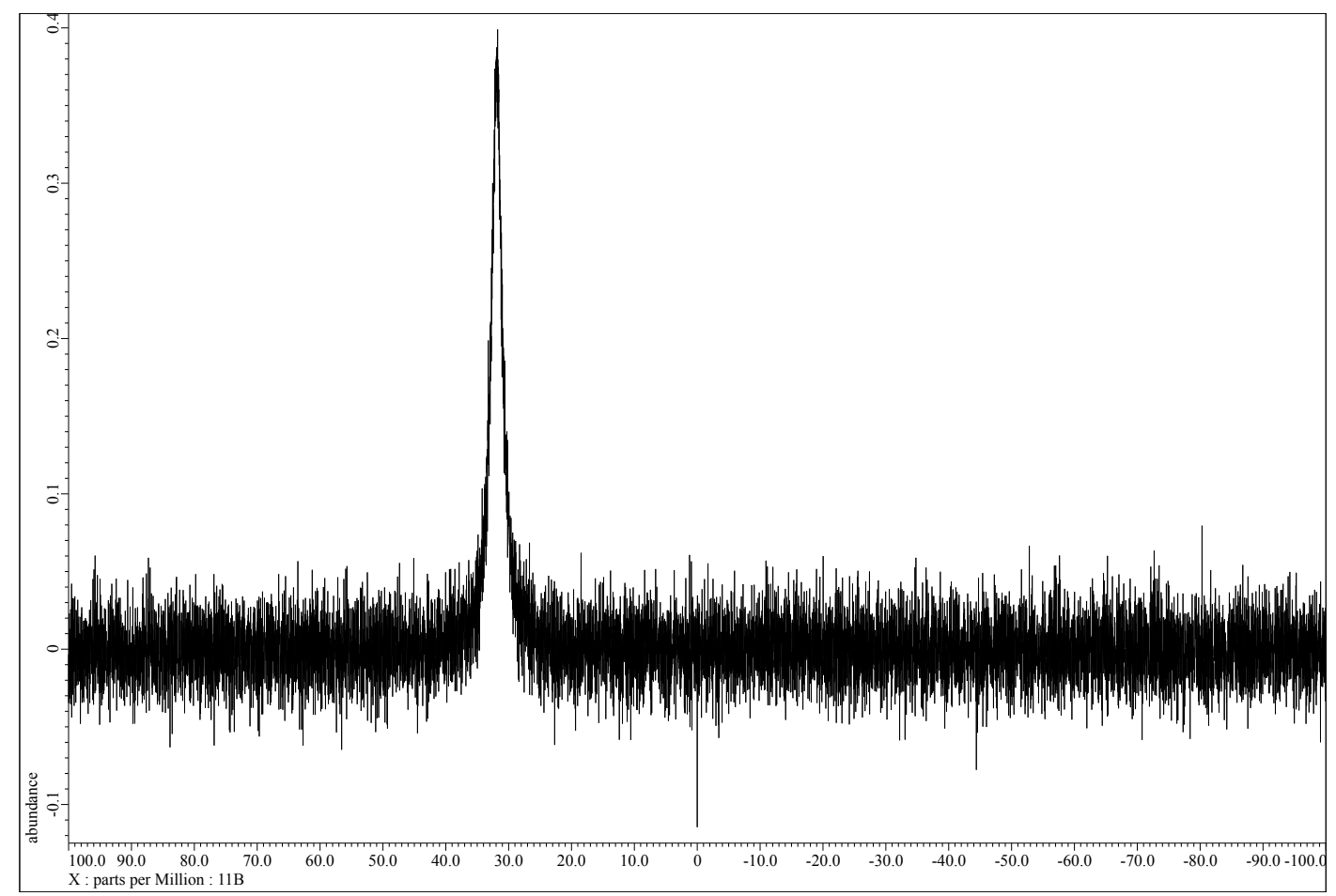

Figure S43. ${ }^{11} \mathrm{~B}$ NMR spectrum of $\mathbf{2 m}$ in $\mathrm{CDCl}_{3}$ at $25{ }^{\circ} \mathrm{C}$. 


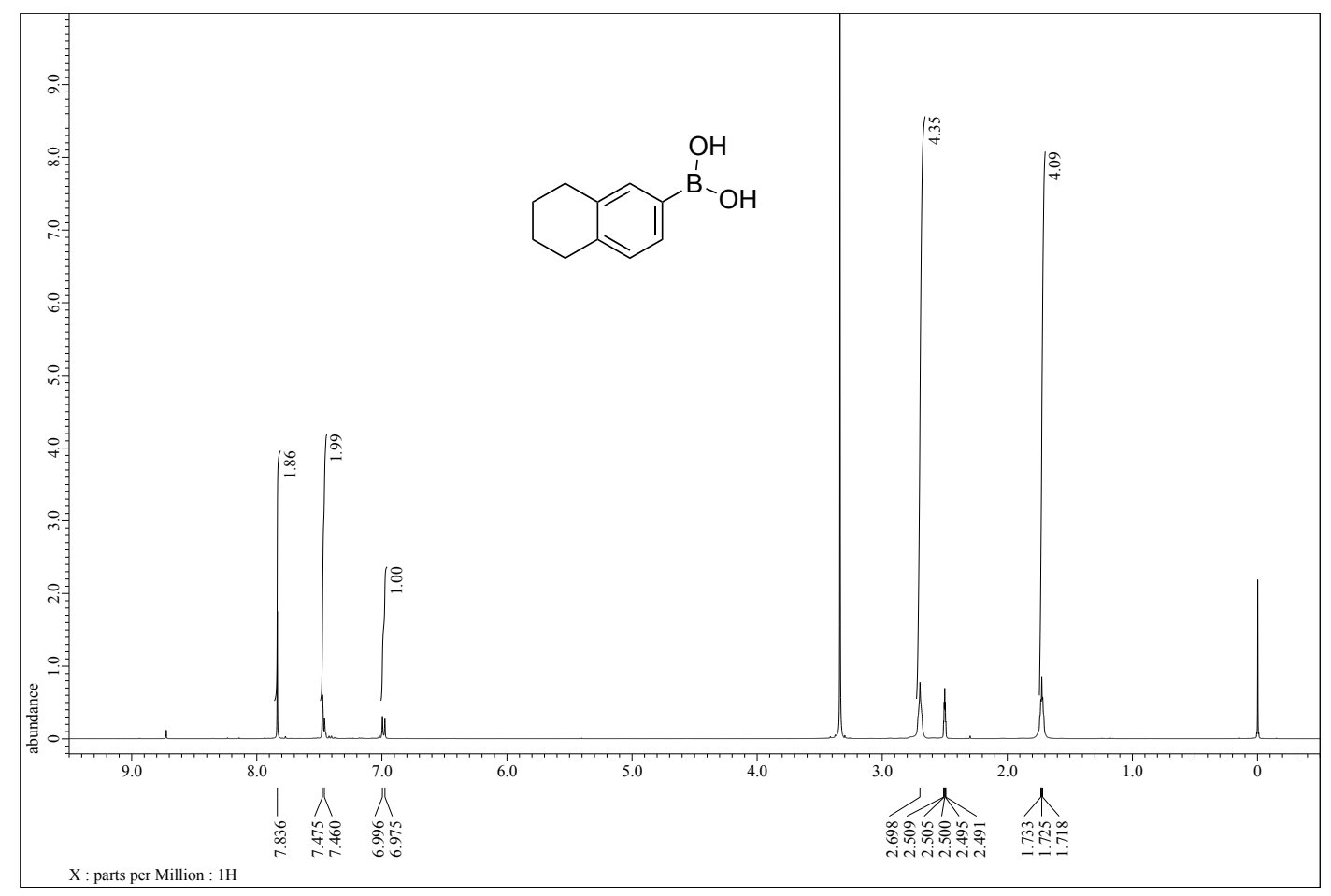

Figure S44. ${ }^{1} \mathrm{H}$ NMR spectrum of 3 in $\left(\mathrm{CD}_{3}\right)_{2} \mathrm{SO}$ at $25{ }^{\circ} \mathrm{C}$.

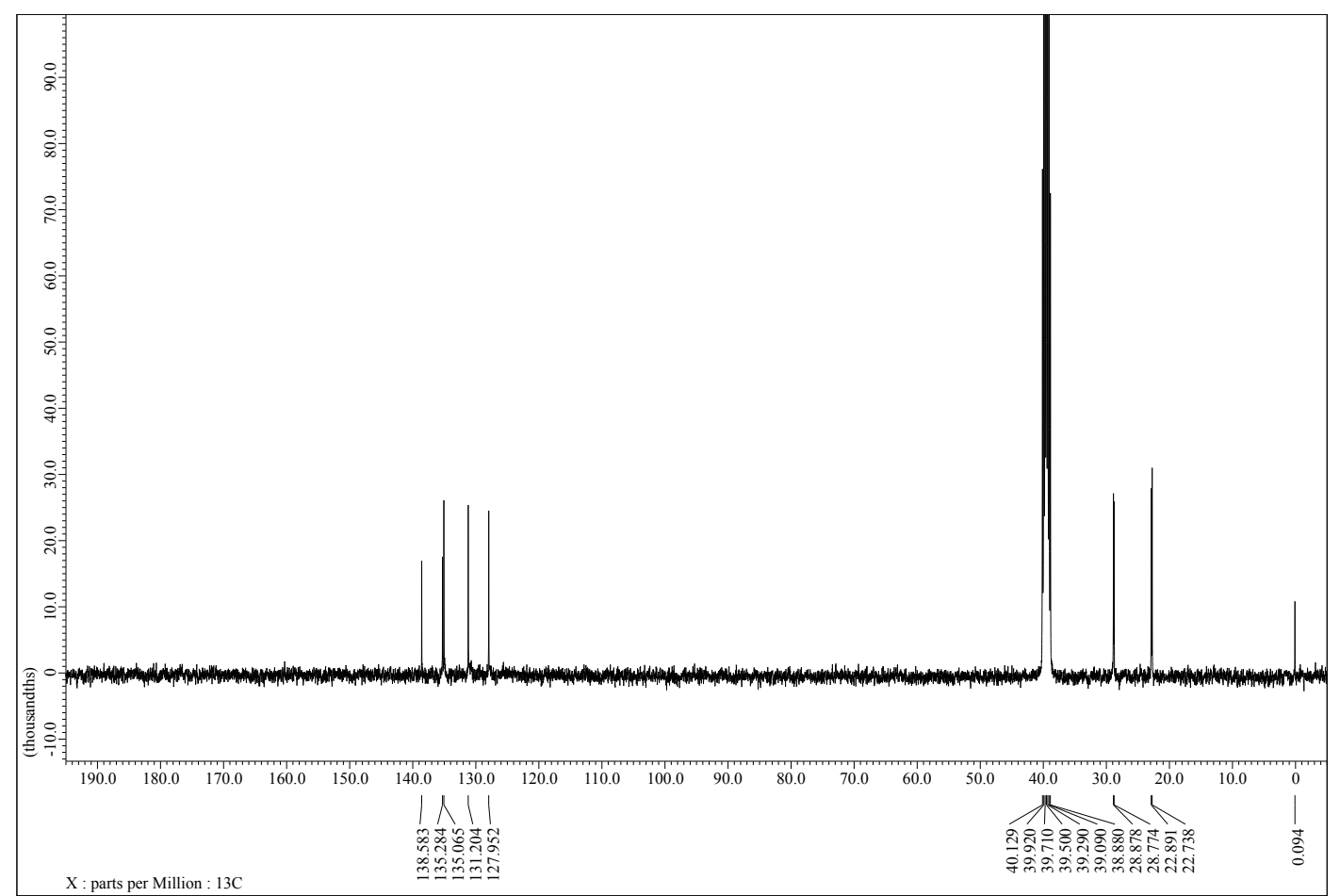

Figure S45. ${ }^{13} \mathrm{C}$ NMR spectrum of 3 in $\left(\mathrm{CD}_{3}\right)_{2} \mathrm{SO}$ at $25{ }^{\circ} \mathrm{C}$. 


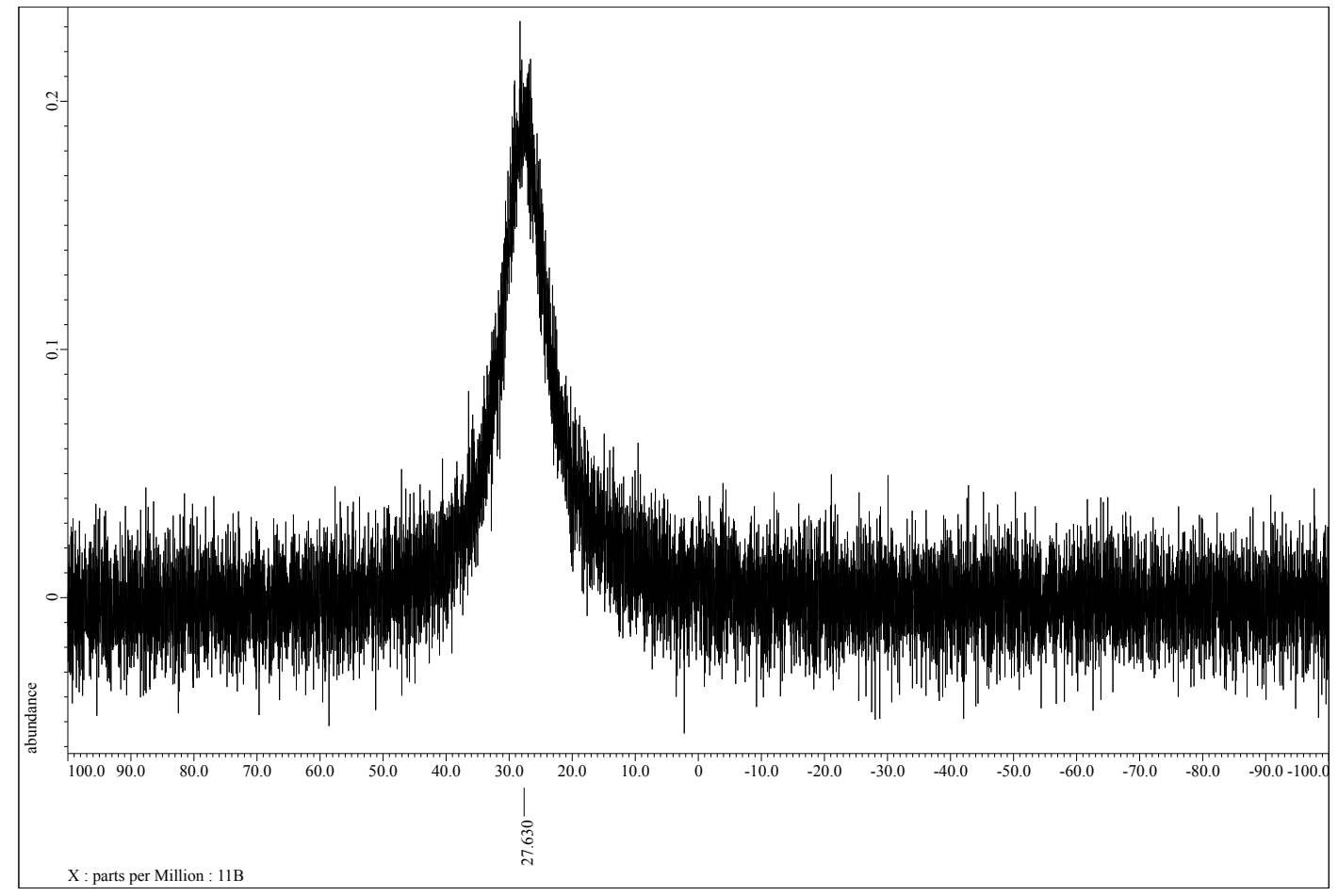

Figure S46. ${ }^{11} \mathrm{~B}$ NMR spectrum of 3 in $\left(\mathrm{CD}_{3}\right)_{2} \mathrm{SO}$ at $25{ }^{\circ} \mathrm{C}$.

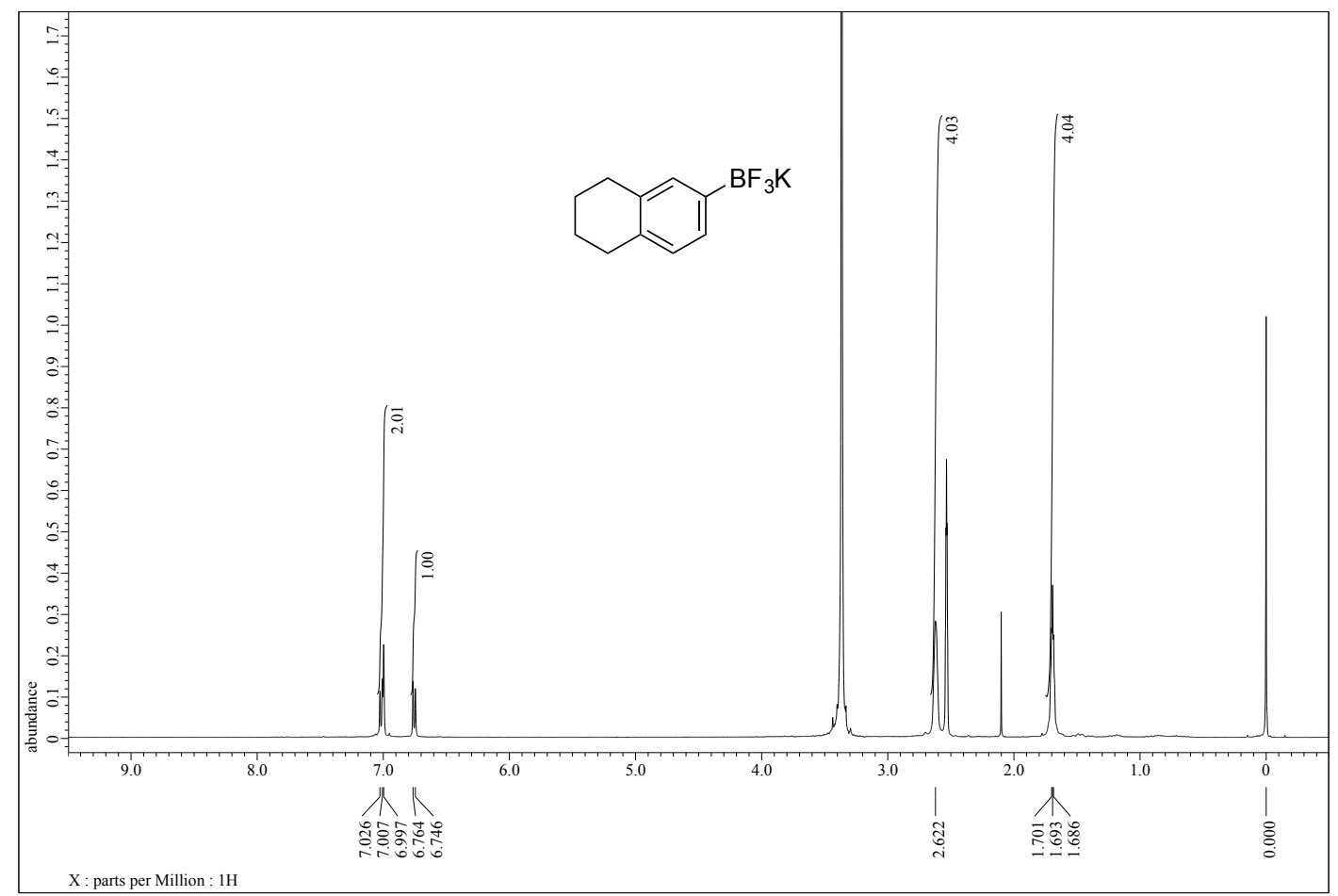

Figure S47. ${ }^{1} \mathrm{H}$ NMR spectrum of 4 in $\left(\mathrm{CD}_{3}\right)_{2} \mathrm{CO}$ at $25{ }^{\circ} \mathrm{C}$. 


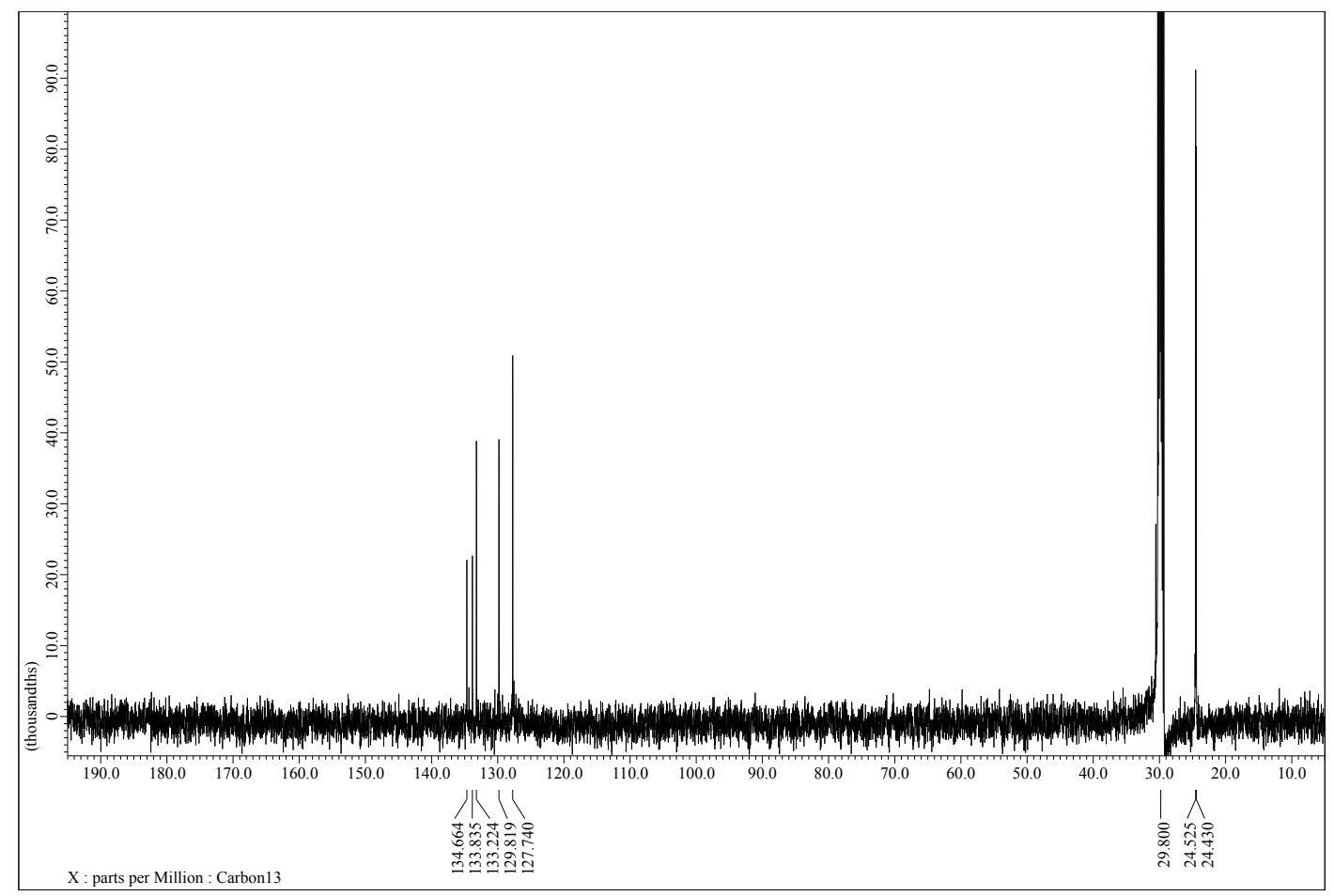

Figure S48. ${ }^{13} \mathrm{C}$ NMR spectrum of 4 in $\left(\mathrm{CD}_{3}\right)_{2} \mathrm{CO}$ at $25{ }^{\circ} \mathrm{C}$.

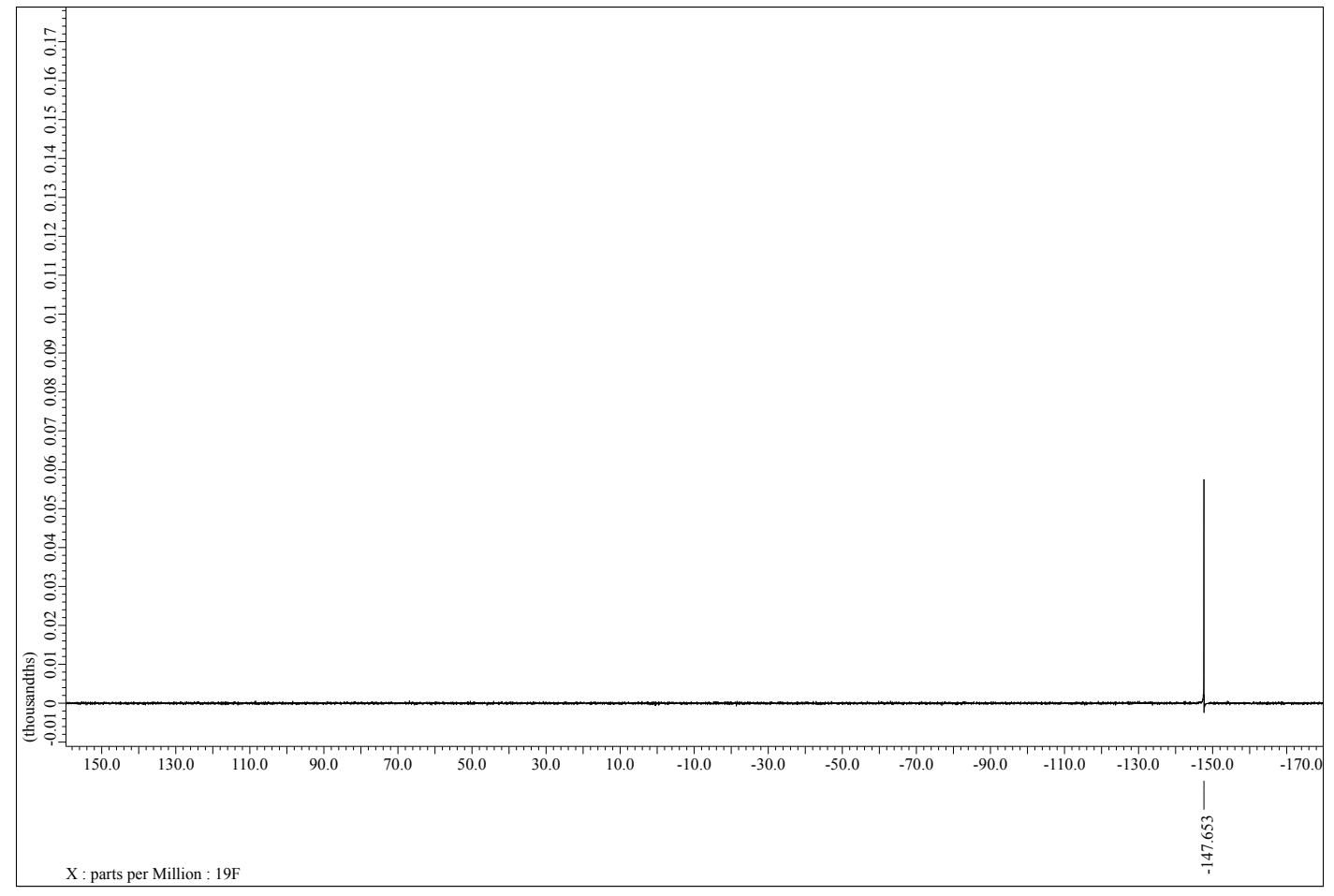

Figure S49. ${ }^{19} \mathrm{~F}$ NMR spectrum of 4 in $\left(\mathrm{CD}_{3}\right)_{2} \mathrm{CO}$ at $25{ }^{\circ} \mathrm{C}$. 


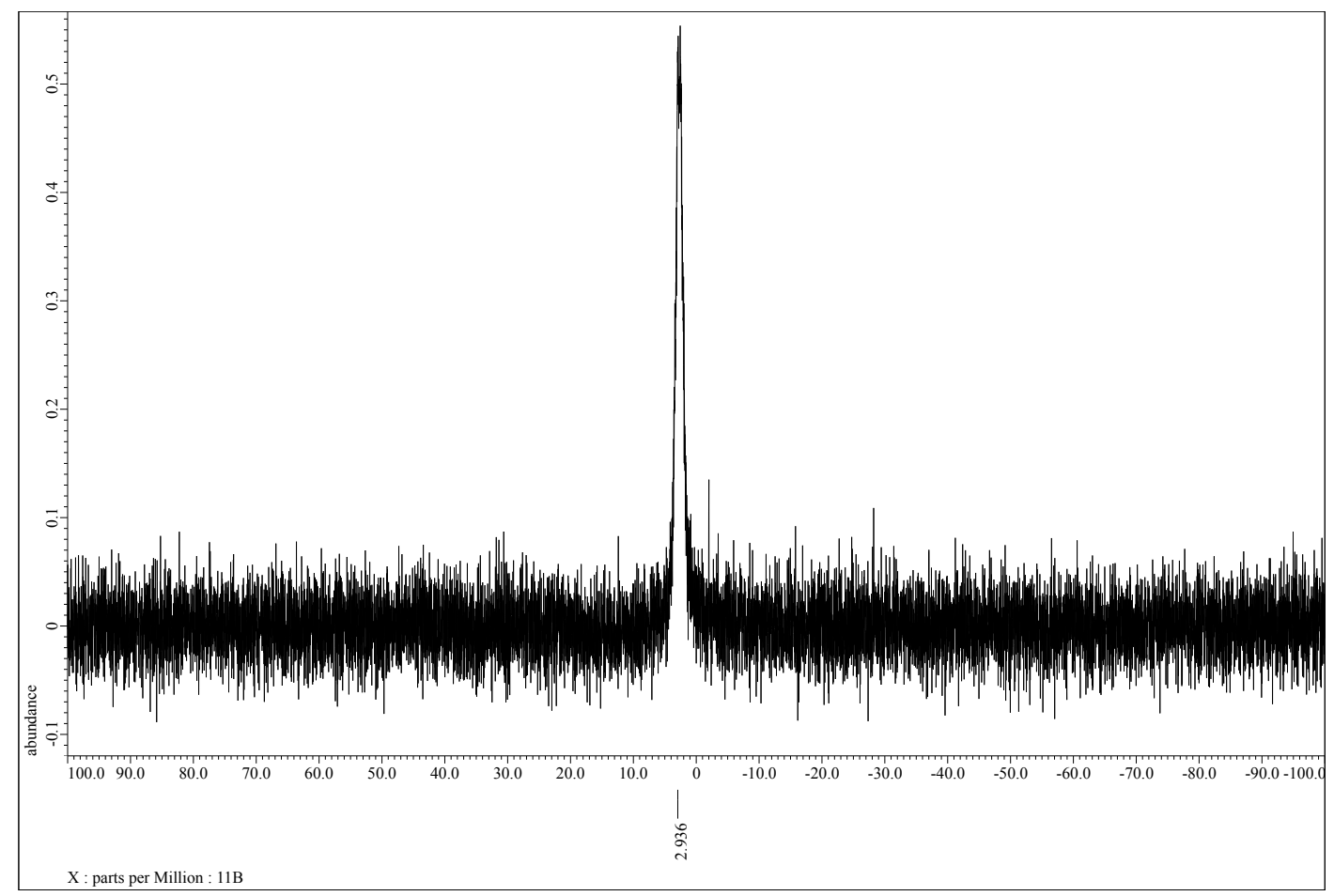

Figure S50. ${ }^{11} \mathrm{~B}$ NMR spectrum of 4 in $\left(\mathrm{CD}_{3}\right)_{2} \mathrm{CO}$ at $25{ }^{\circ} \mathrm{C}$.

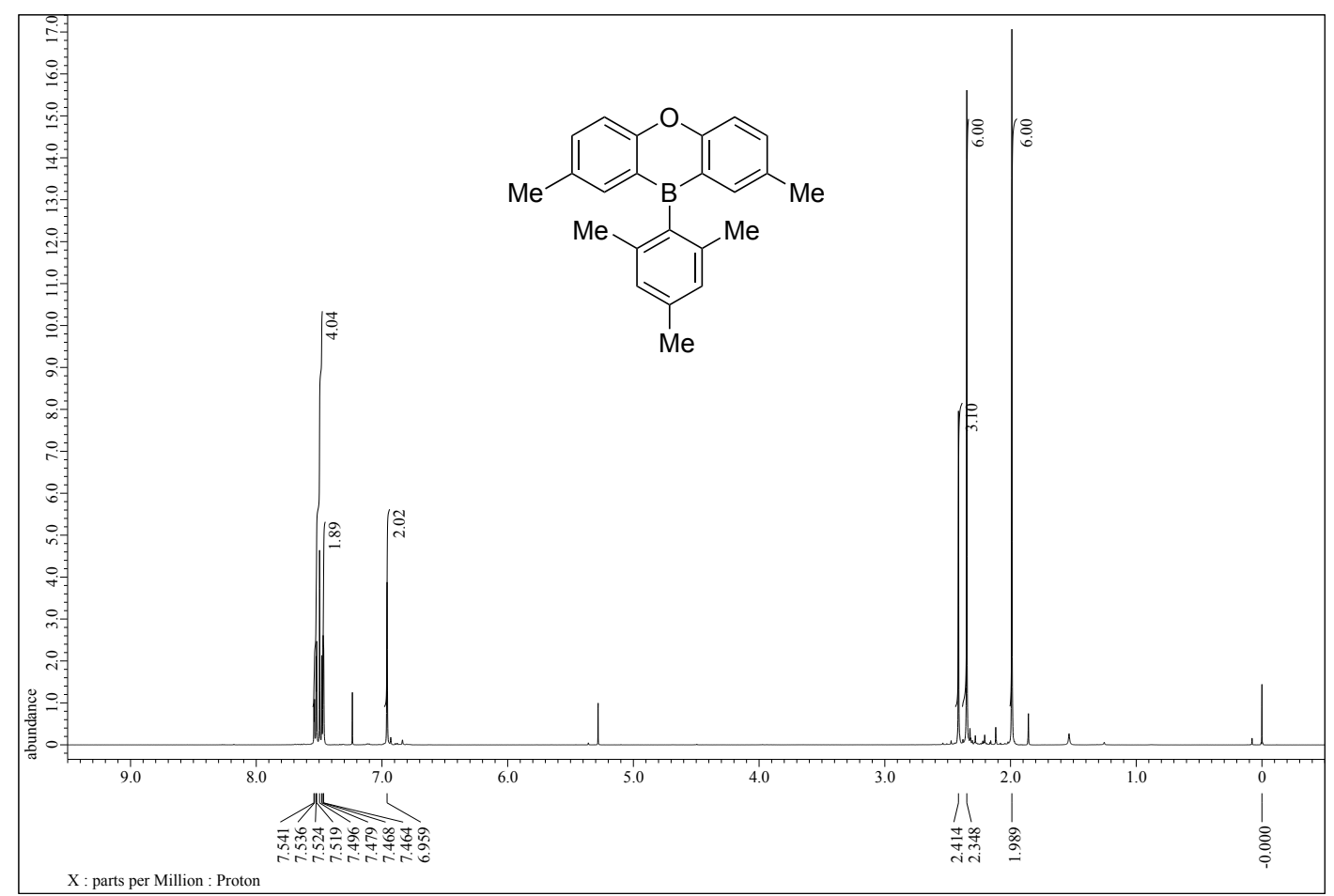

Figure S51. ${ }^{1} \mathrm{H}$ NMR spectrum of 5 in $\mathrm{CDCl}_{3}$ at $25^{\circ} \mathrm{C}$. 


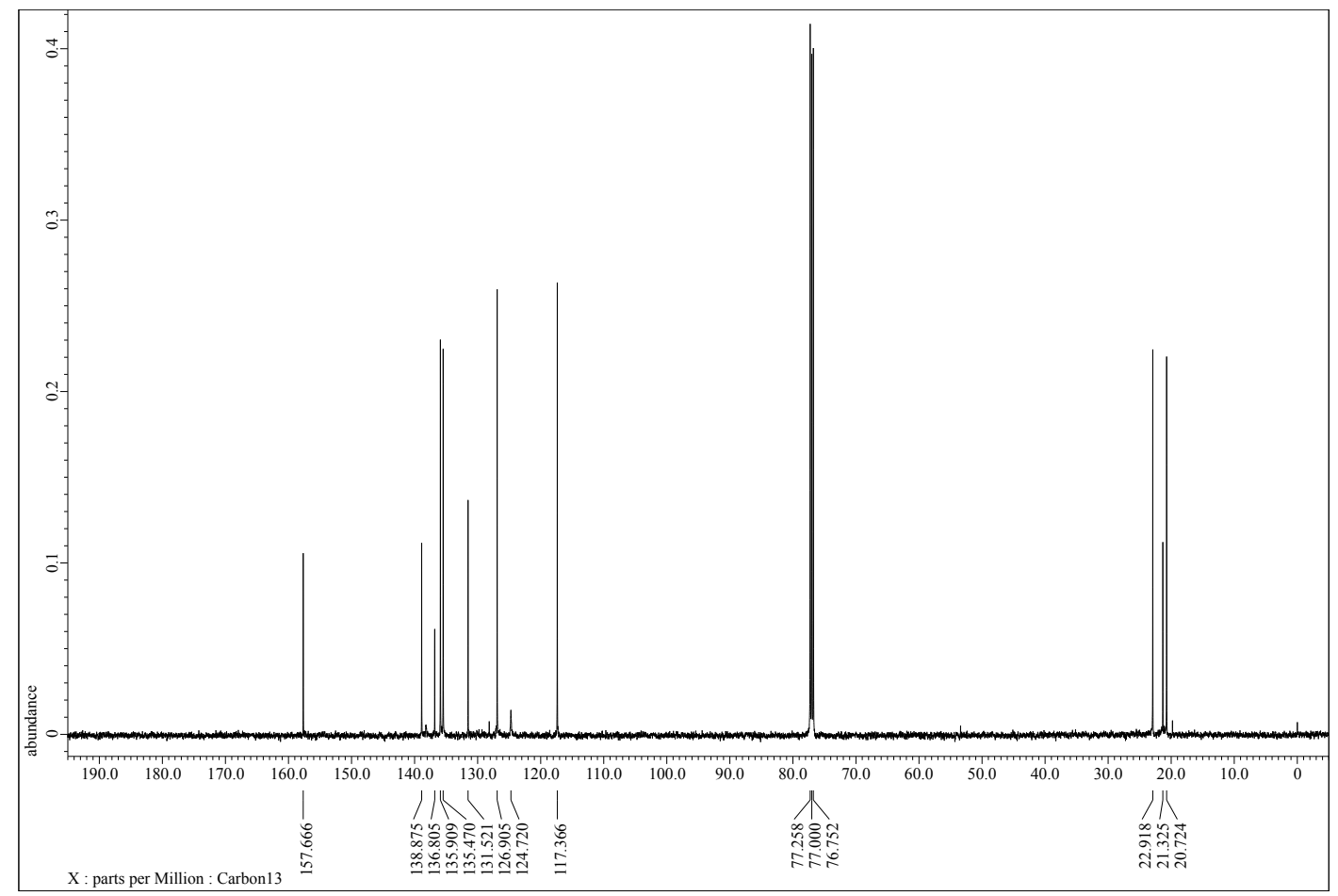

Figure S52. ${ }^{13} \mathrm{C}$ NMR spectrum of 5 in $\mathrm{CDCl}_{3}$ at $25{ }^{\circ} \mathrm{C}$.

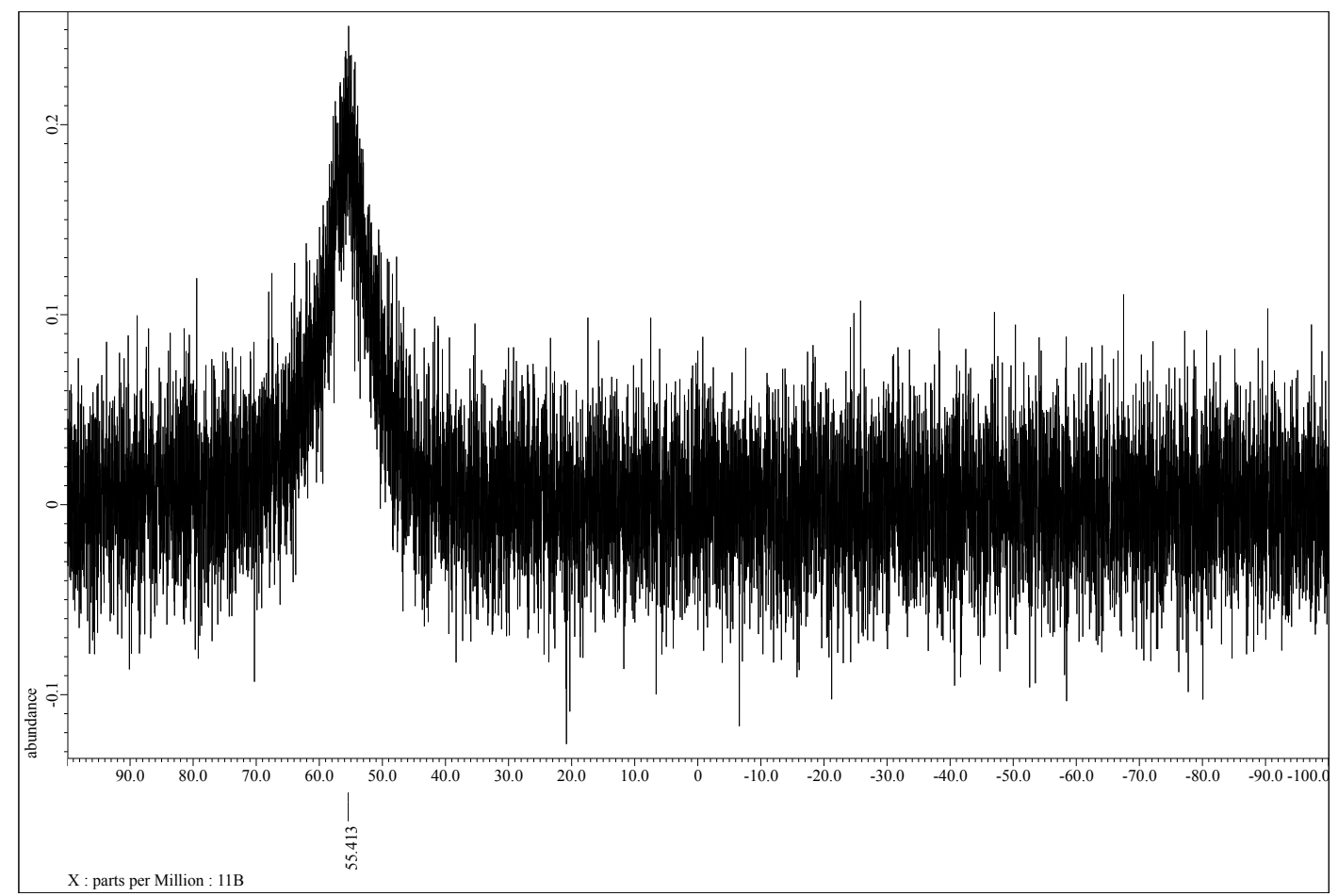

Figure S53. ${ }^{11} \mathrm{~B}$ NMR spectrum of 5 in $\mathrm{CDCl}_{3}$ at $25{ }^{\circ} \mathrm{C}$. 


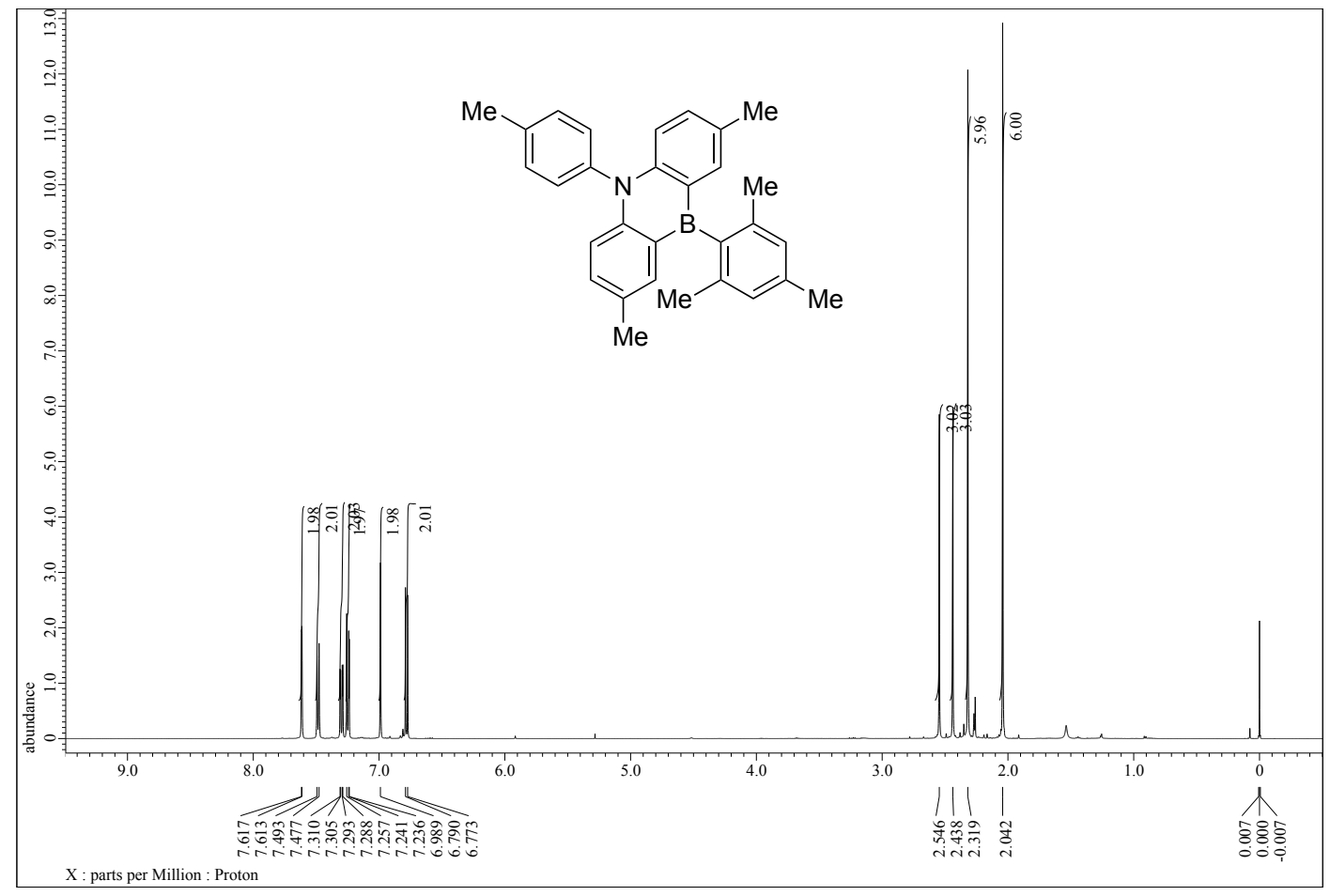

Figure S54. ${ }^{1} \mathrm{H}$ NMR spectrum of 6 in $\mathrm{CDCl}_{3}$ at $25{ }^{\circ} \mathrm{C}$.

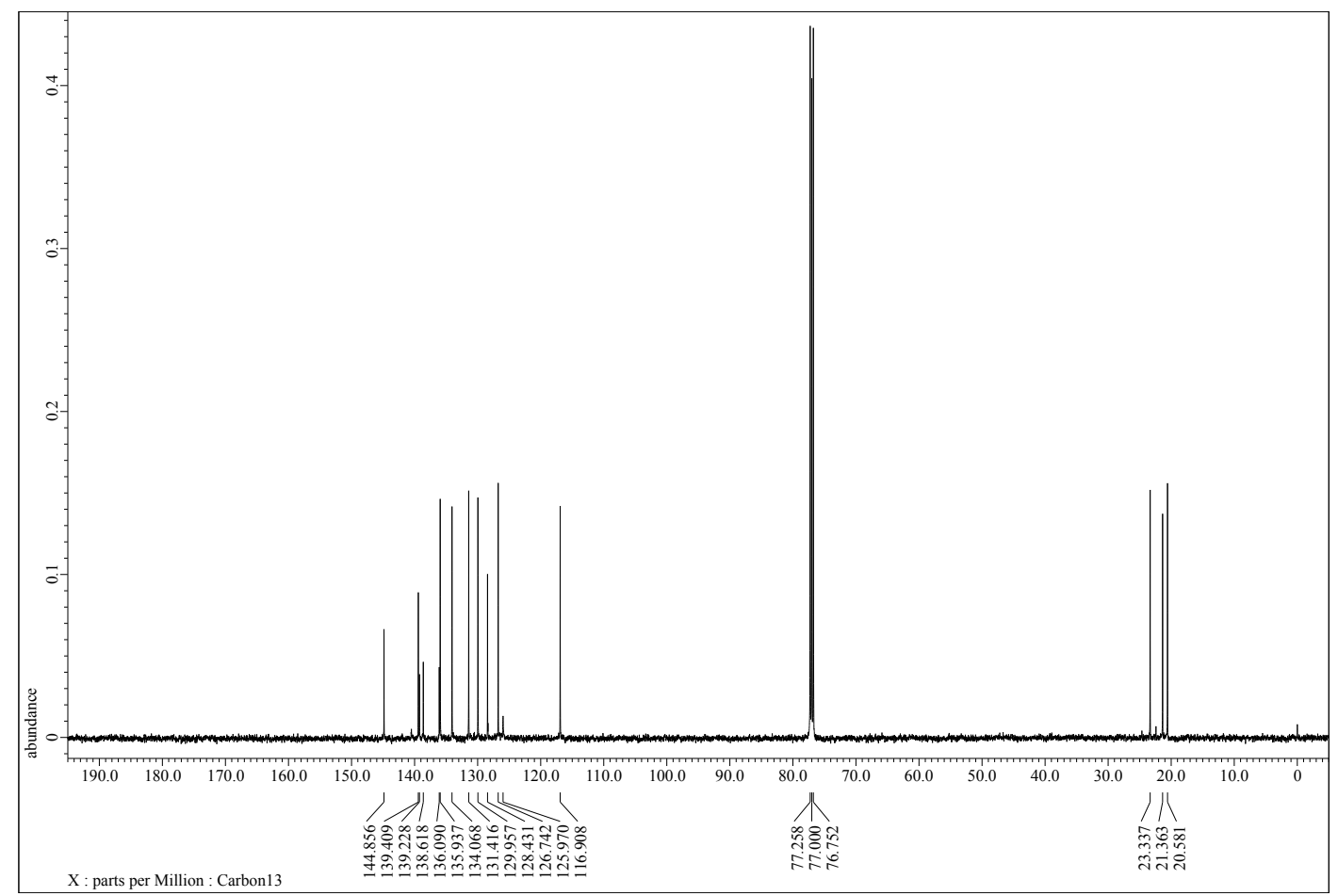

Figure S55. ${ }^{13} \mathrm{C}$ NMR spectrum of 6 in $\mathrm{CDCl}_{3}$ at $25{ }^{\circ} \mathrm{C}$. 


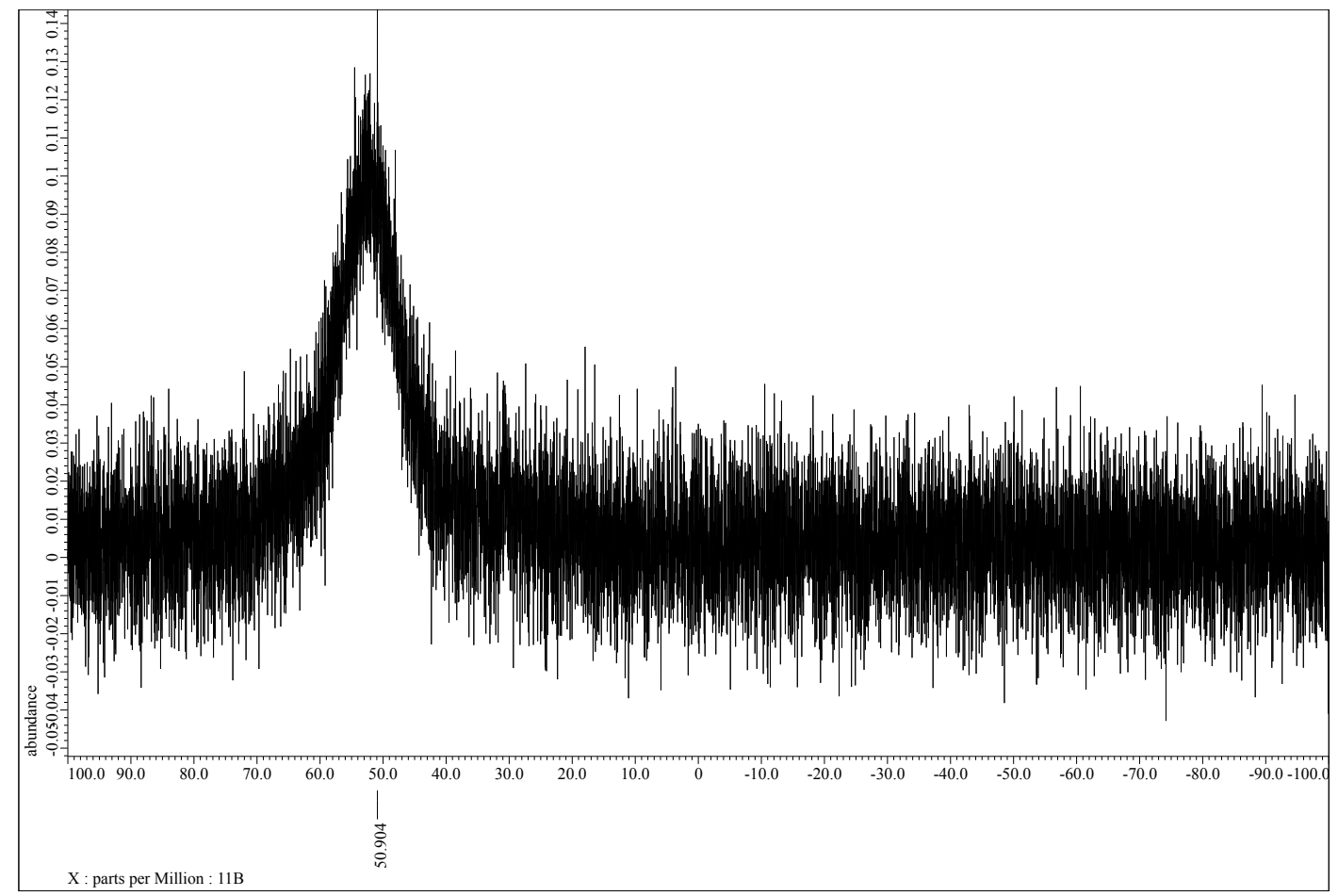

Figure S56. ${ }^{11} \mathrm{~B}$ NMR spectrum of 6 in $\mathrm{CDCl}_{3}$ at $25^{\circ} \mathrm{C}$.

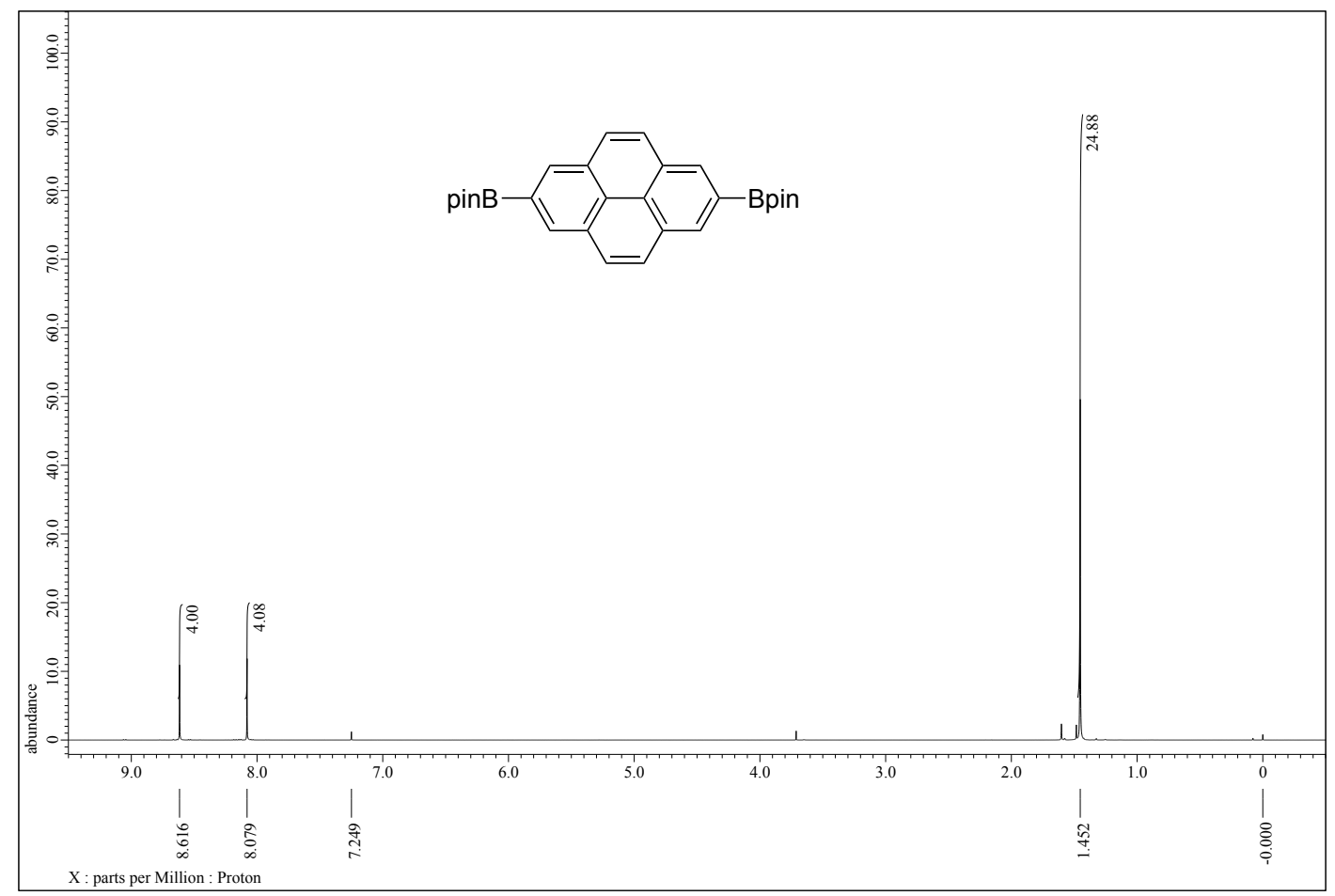

Figure S57. ${ }^{1} \mathrm{H}$ NMR spectrum of 7 in $\mathrm{CDCl}_{3}$ at $25^{\circ} \mathrm{C}$. 


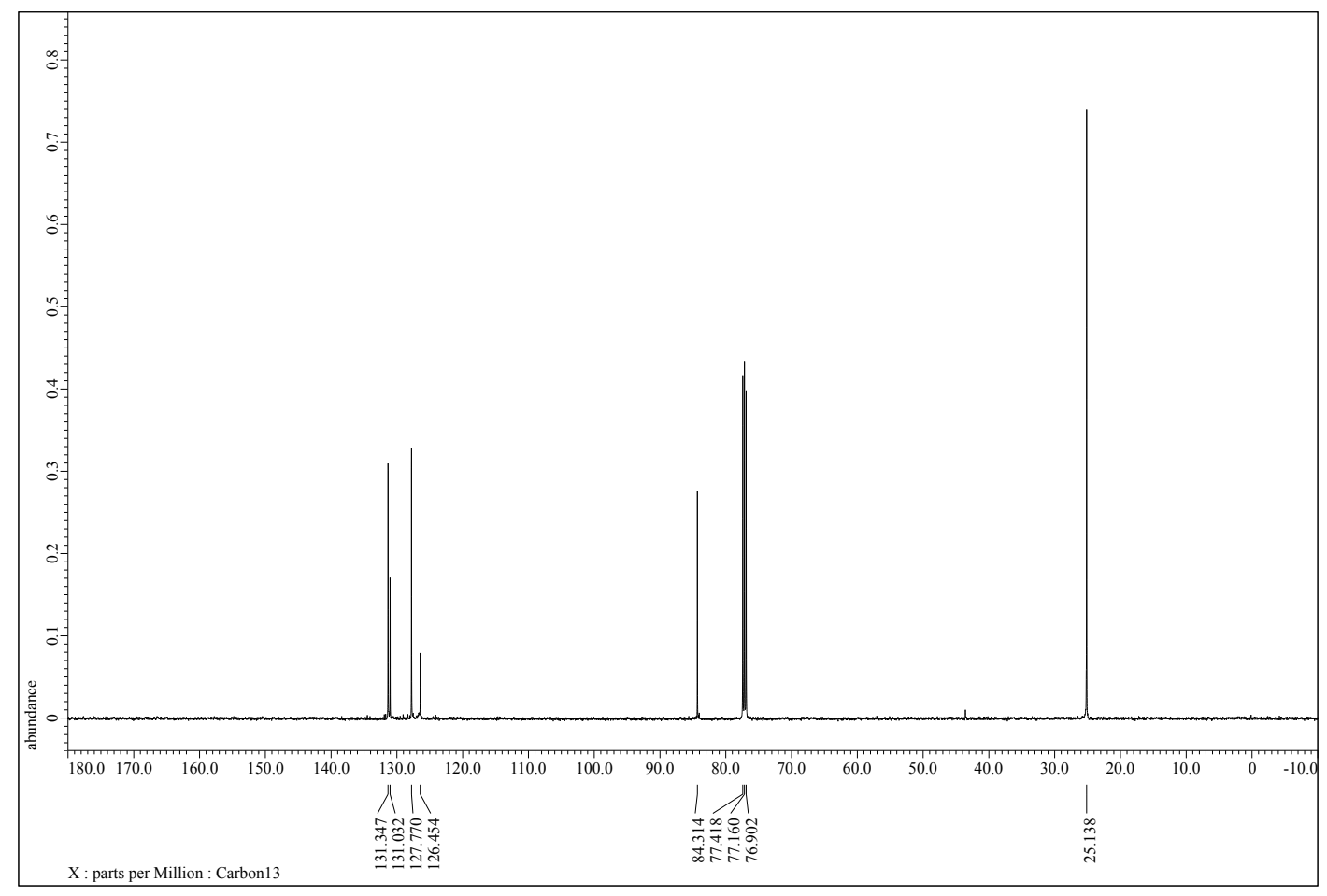

Figure S58. ${ }^{13} \mathrm{C}$ NMR spectrum of 7 in $\mathrm{CDCl}_{3}$ at $25{ }^{\circ} \mathrm{C}$.

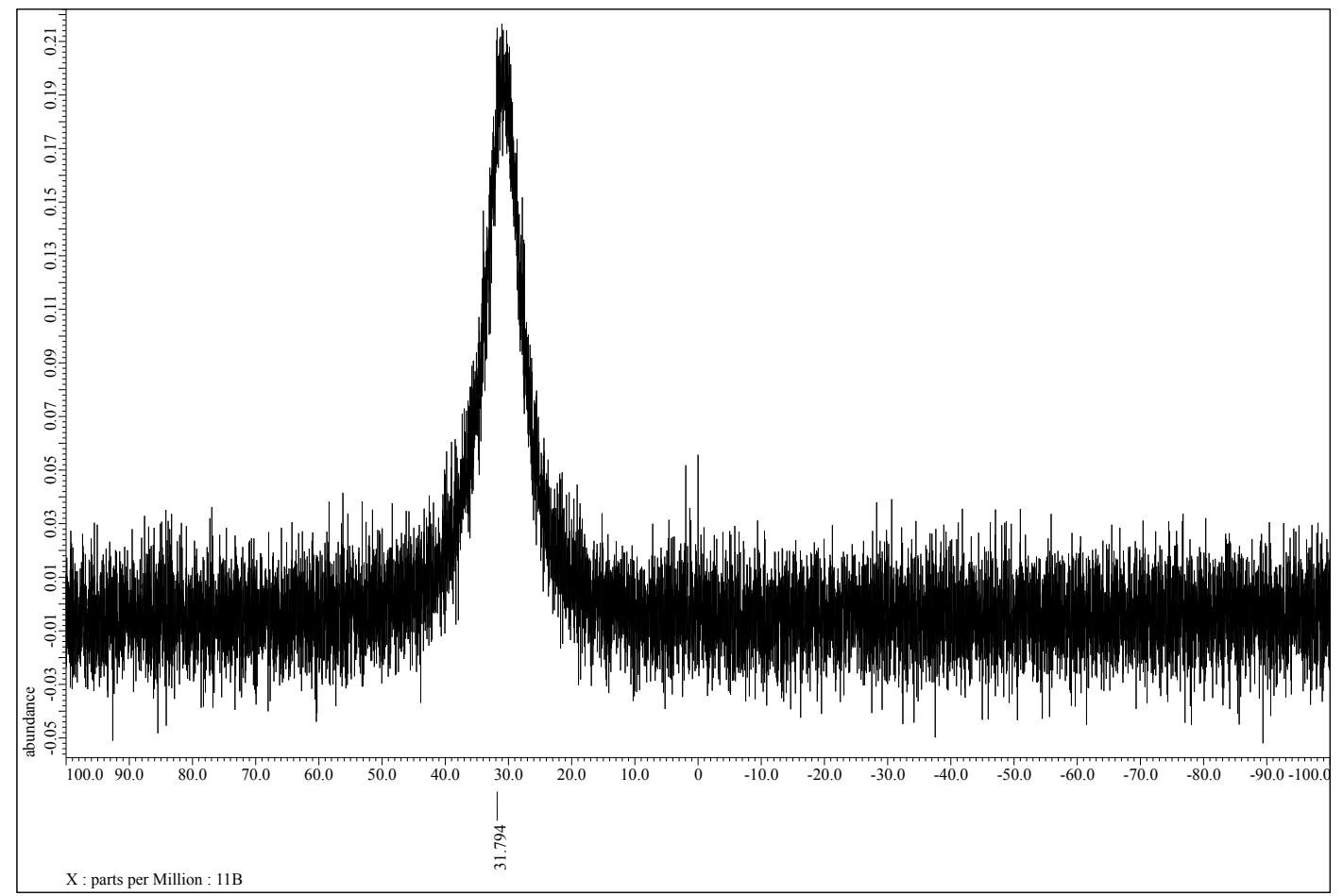

Figure S59. ${ }^{11} \mathrm{~B}$ NMR spectrum of 7 in $\mathrm{CDCl}_{3}$ at $25^{\circ} \mathrm{C}$. 


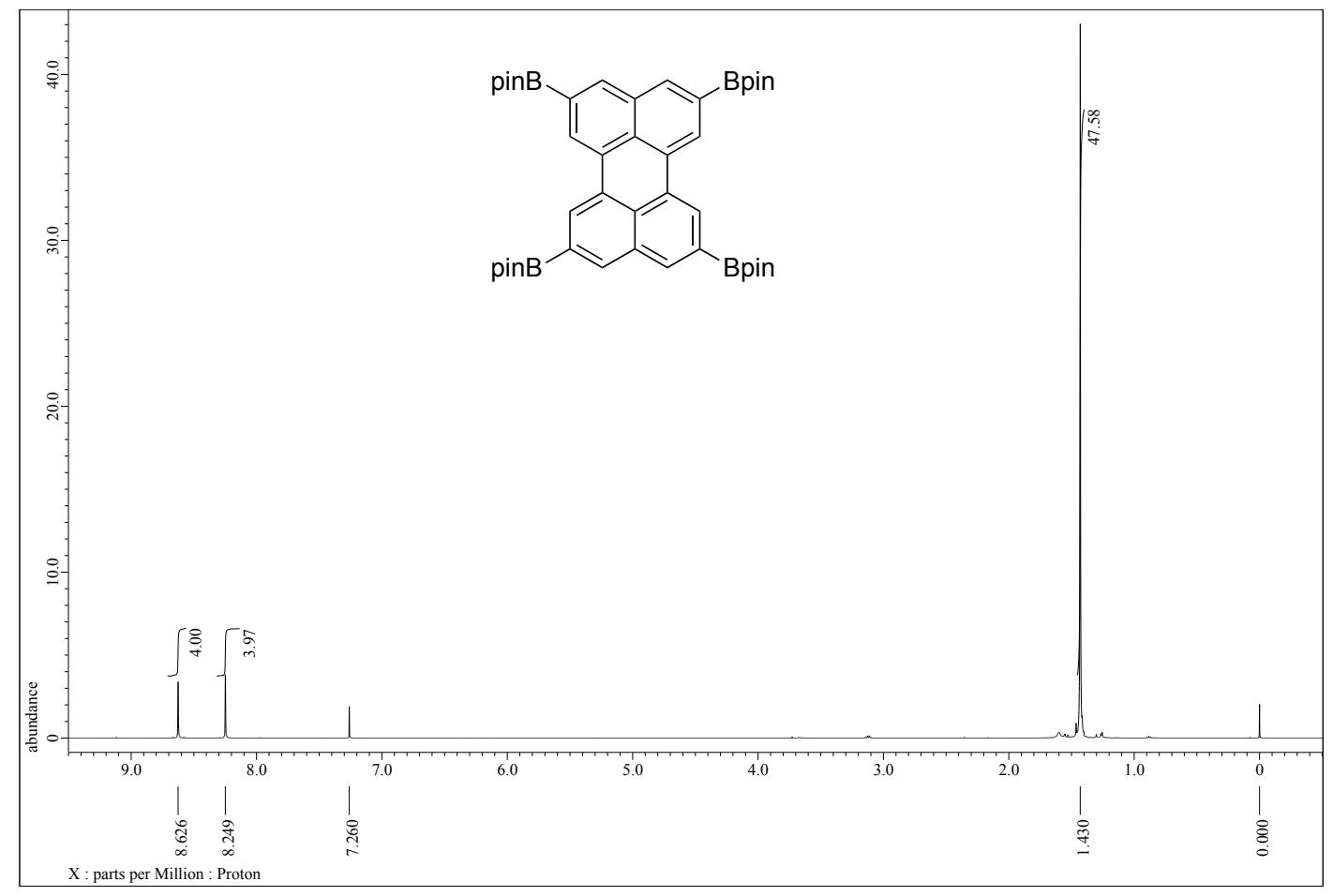

Figure S60. ${ }^{1} \mathrm{H}$ NMR spectrum of 8 in $\mathrm{CDCl}_{3}$ at $25{ }^{\circ} \mathrm{C}$.

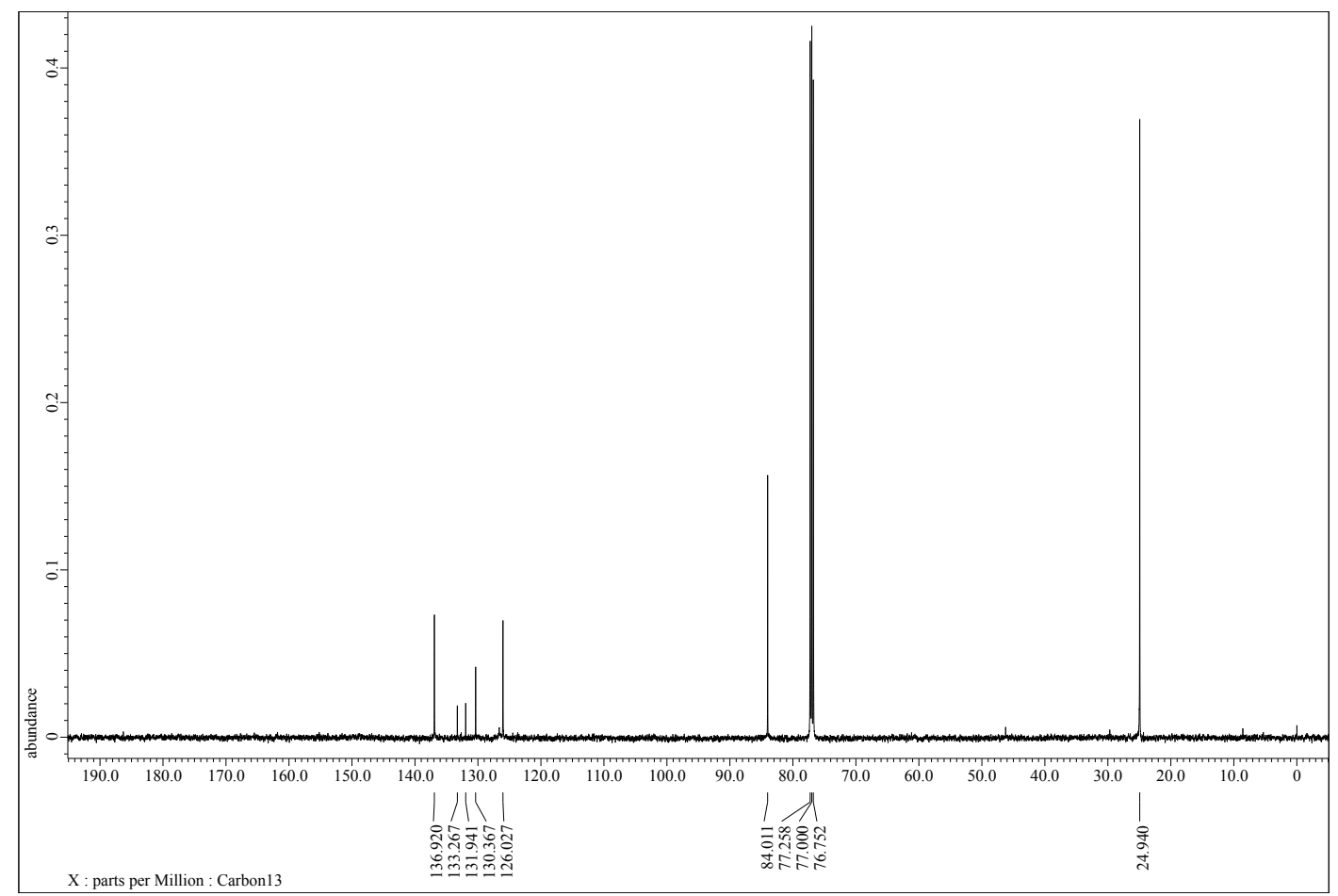

Figure S61. ${ }^{13} \mathrm{C}$ NMR spectrum of 8 in $\mathrm{CDCl}_{3}$ at $25{ }^{\circ} \mathrm{C}$. 


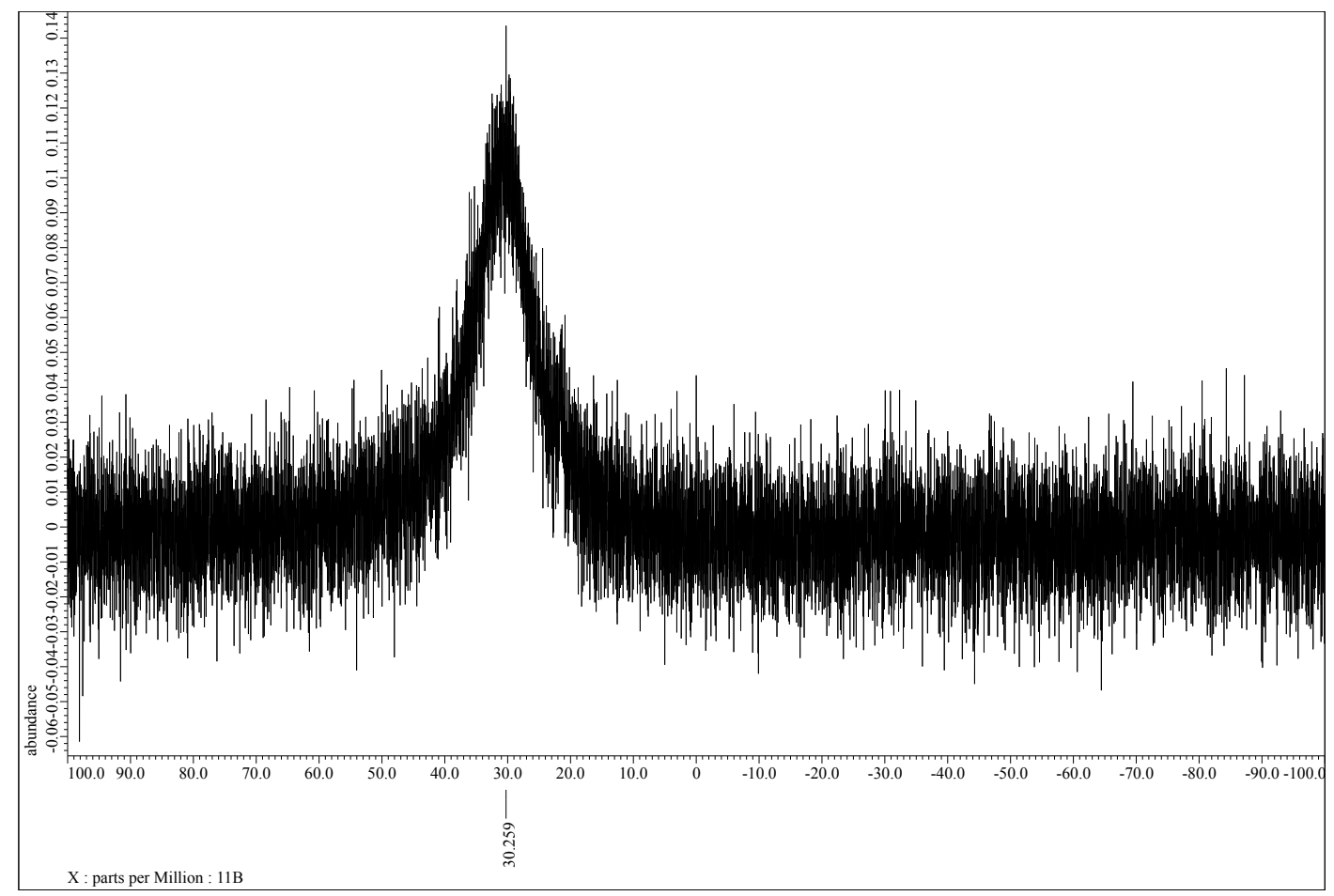

Figure S62. ${ }^{11} \mathrm{~B}$ NMR spectrum of 8 in $\mathrm{CDCl}_{3}$ at $25{ }^{\circ} \mathrm{C}$.

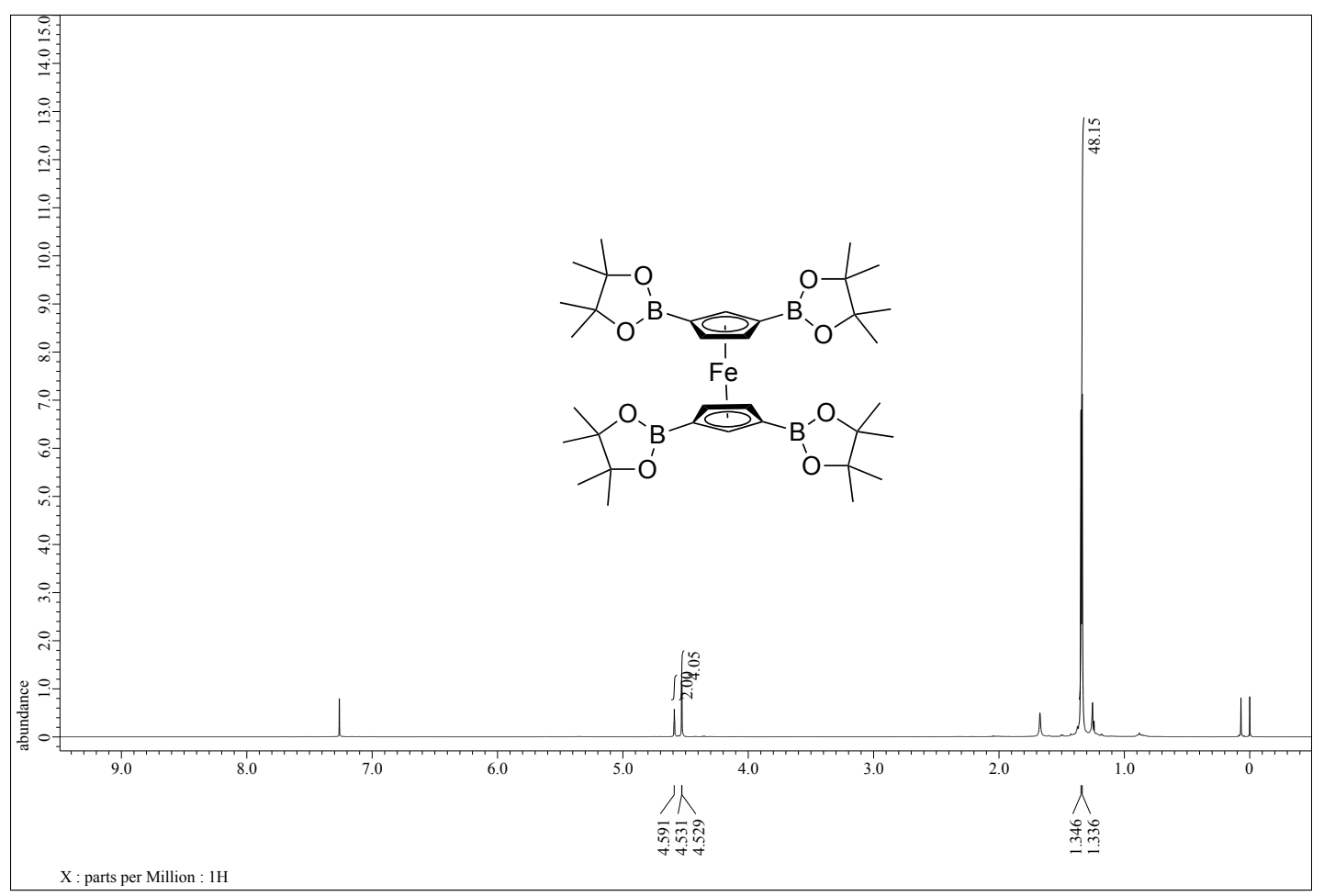

Figure S63. ${ }^{1} \mathrm{H}$ NMR spectrum of 9 in $\mathrm{CDCl}_{3}$ at $25^{\circ} \mathrm{C}$. 


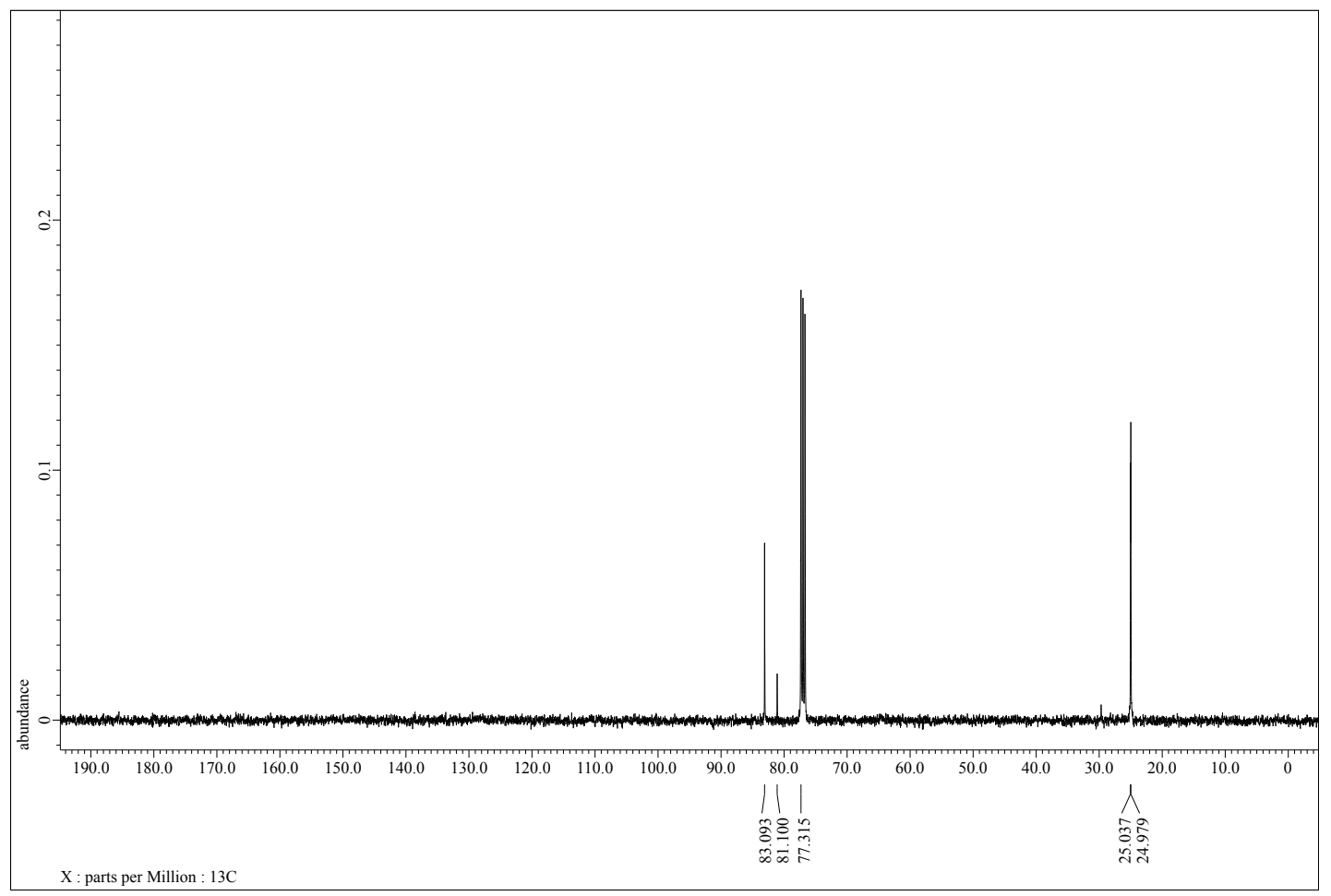

Figure S64. ${ }^{13} \mathrm{C}$ NMR spectrum of 9 in $\mathrm{CDCl}_{3}$ at $25{ }^{\circ} \mathrm{C}$.

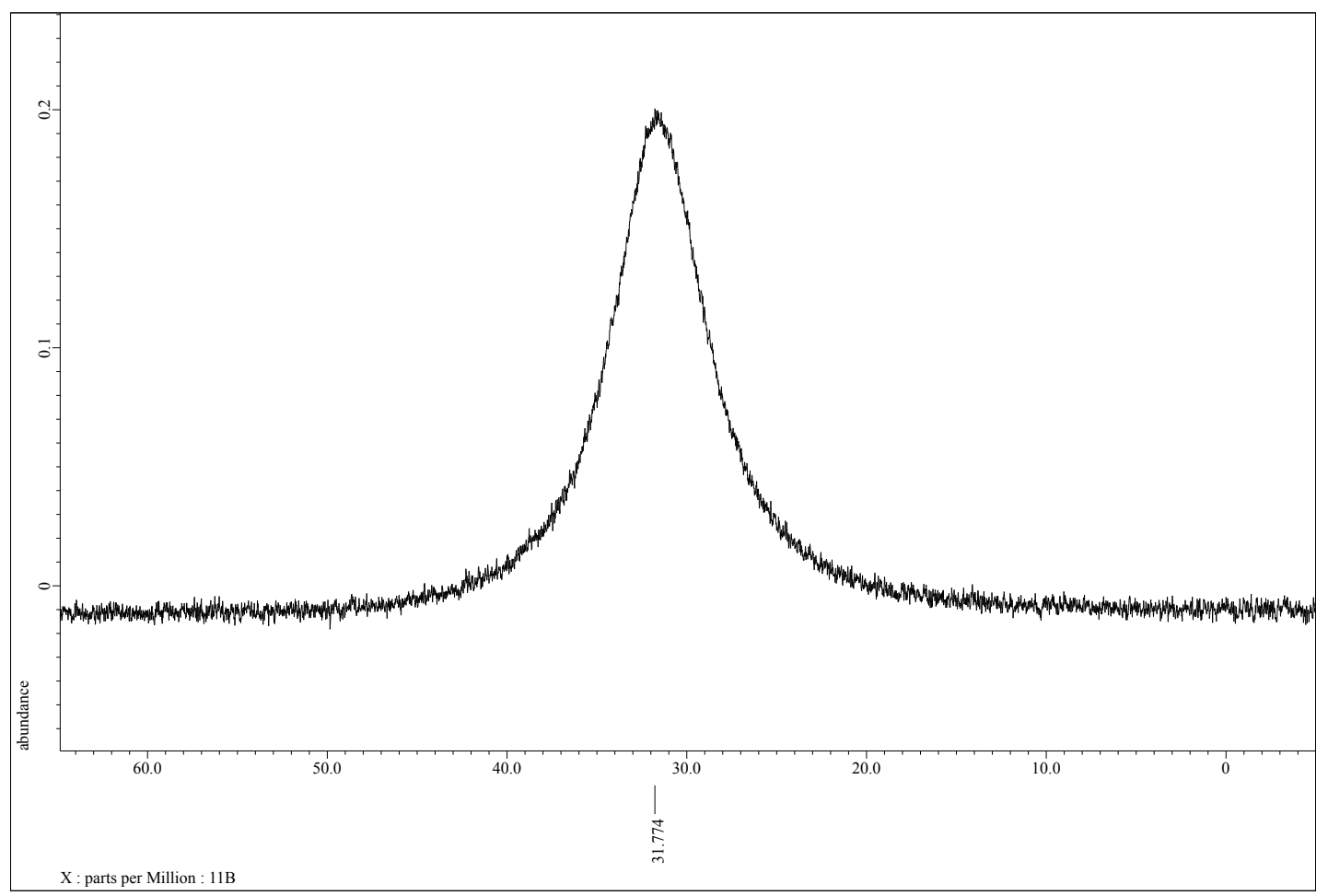

Figure S65. ${ }^{11} \mathrm{~B}$ NMR spectrum of 9 in $\mathrm{CDCl}_{3}$ at $25^{\circ} \mathrm{C}$. 


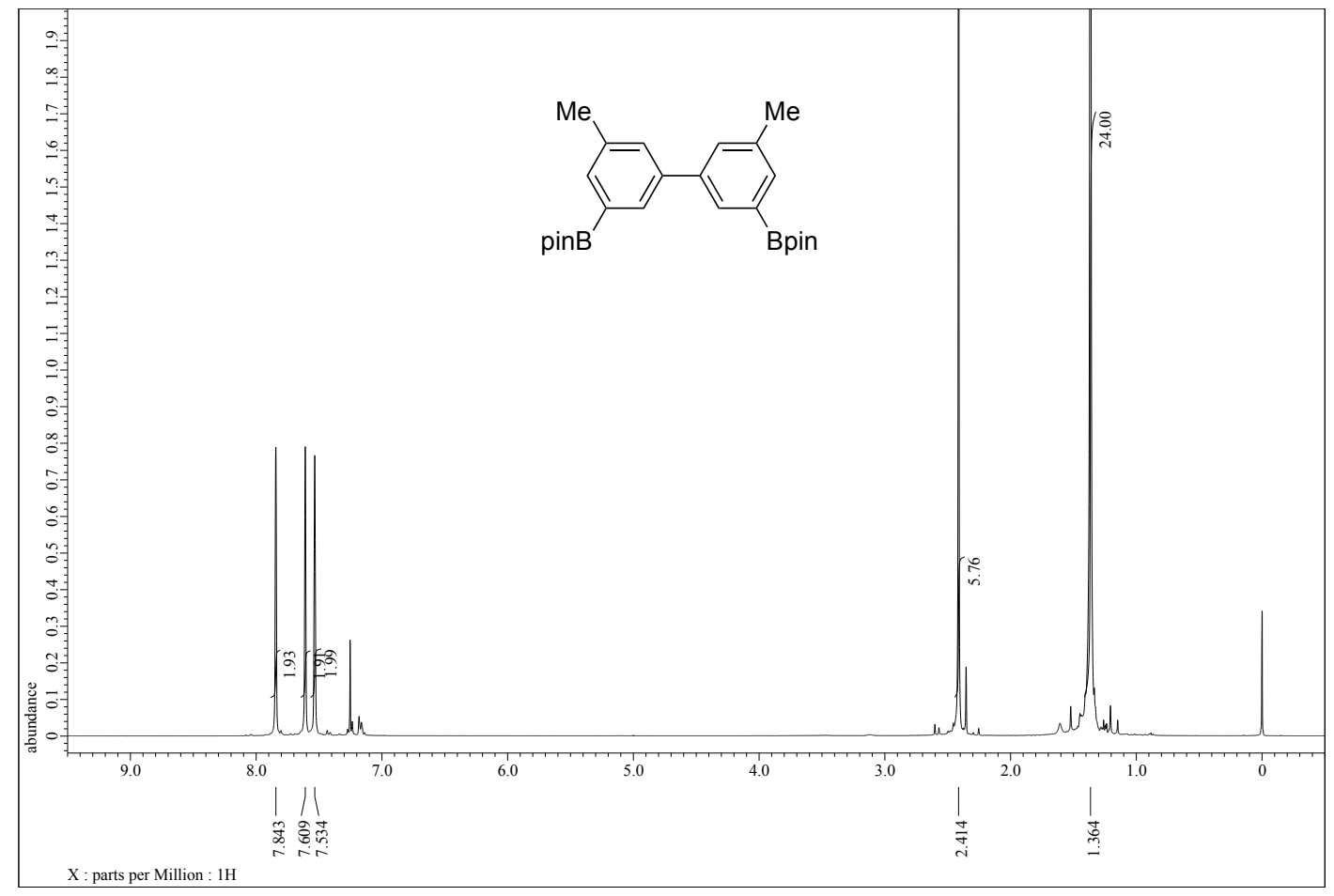

Figure S66. ${ }^{1} \mathrm{H}$ NMR spectrum of 10 in $\mathrm{CDCl}_{3}$ at $25^{\circ} \mathrm{C}$.

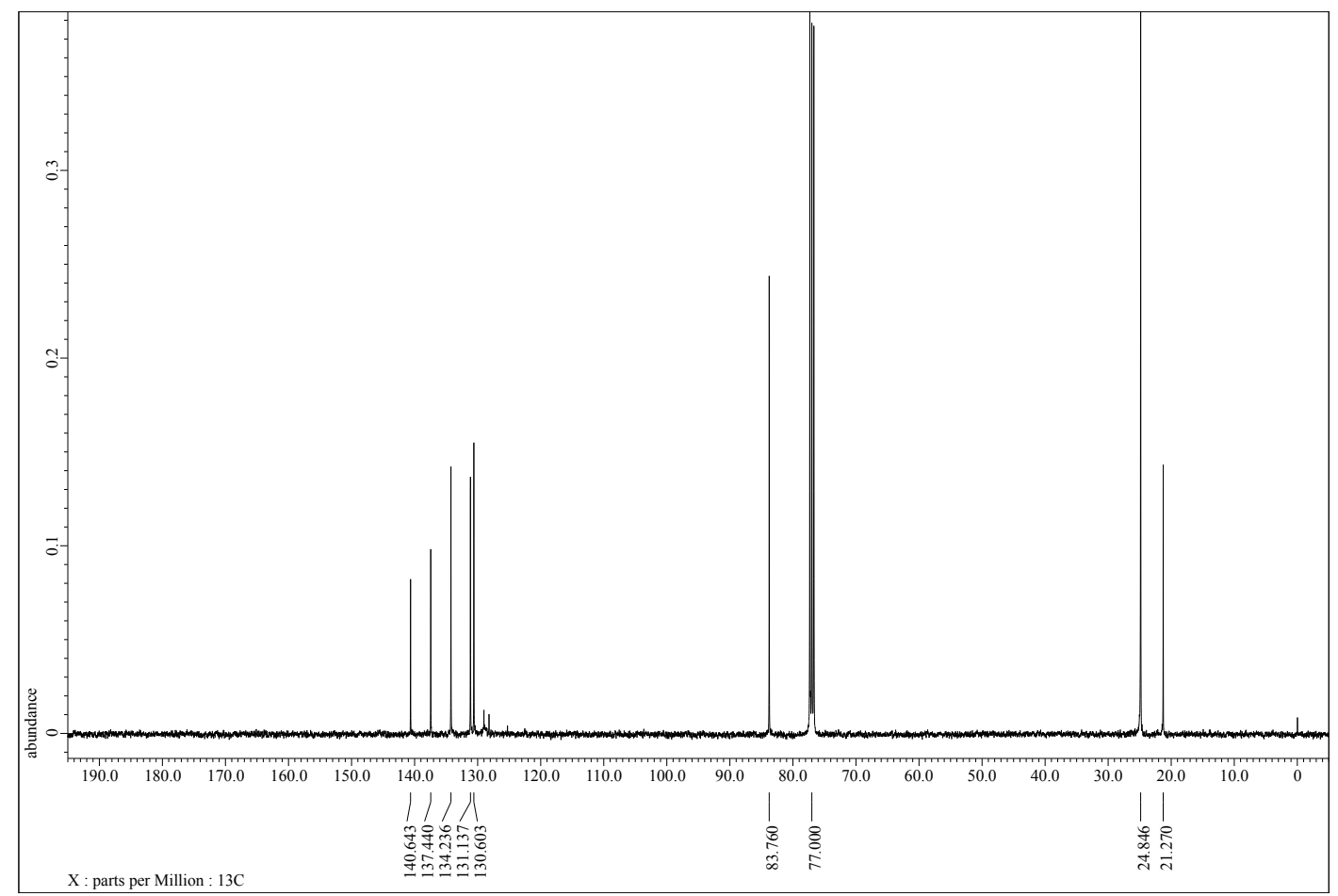

Figure S67. ${ }^{13} \mathrm{C}$ NMR spectrum of $\mathbf{1 0}$ in $\mathrm{CDCl}_{3}$ at $25{ }^{\circ} \mathrm{C}$. 


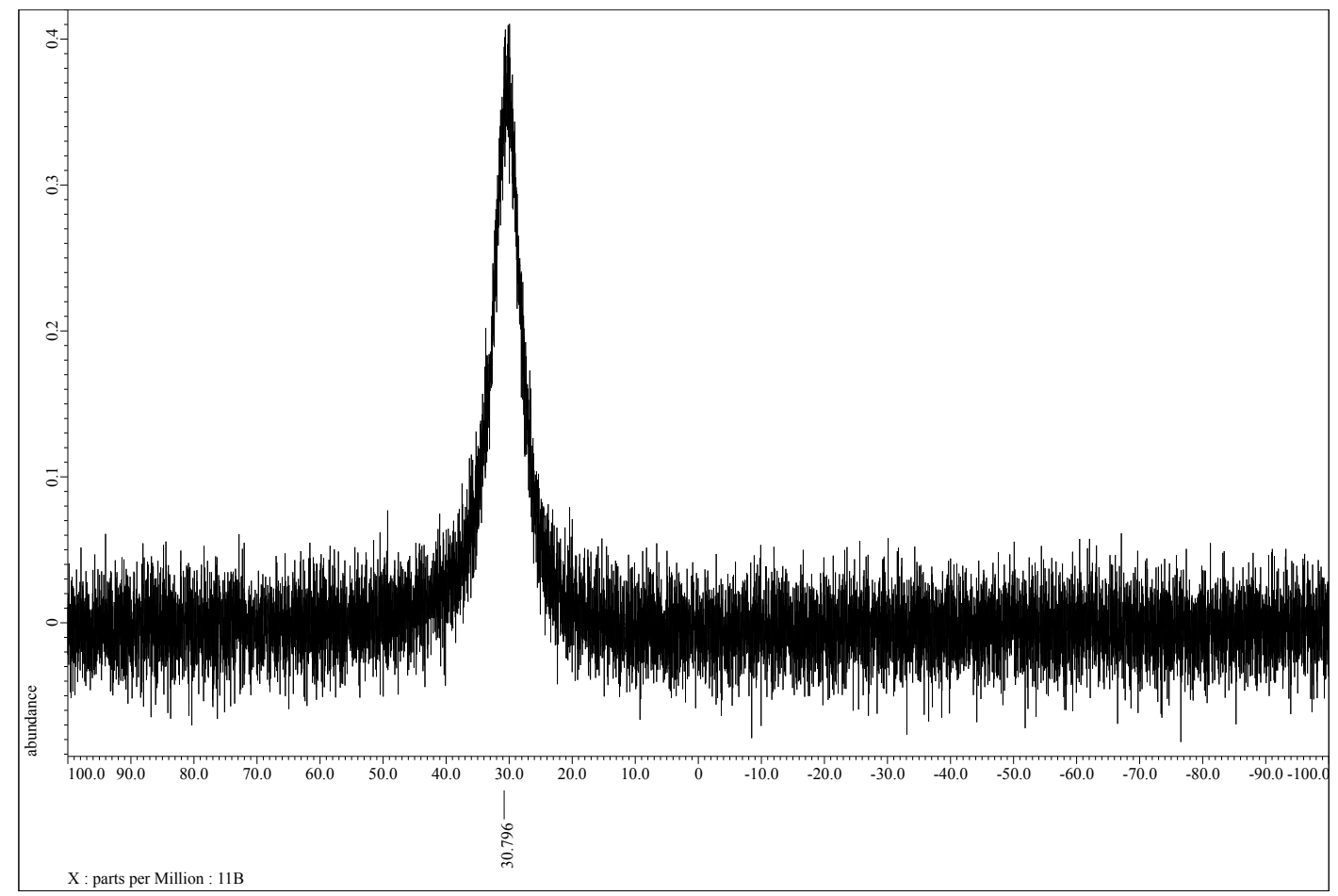

Figure S68. ${ }^{11} \mathrm{~B}$ NMR spectrum of 10 in $\mathrm{CDCl}_{3}$ at $25^{\circ} \mathrm{C}$. 